A Multi-Mode Spectrometer for Atomic Emission Spectrometry

by

Mark A. Wingerd

Dissertation submitted to the Faculty of the

Virginia Polytechnic Institute and State University

in partial fulfillment of the requirements for the degree of

Doctor of Philosophy

in

Department of Chemistry
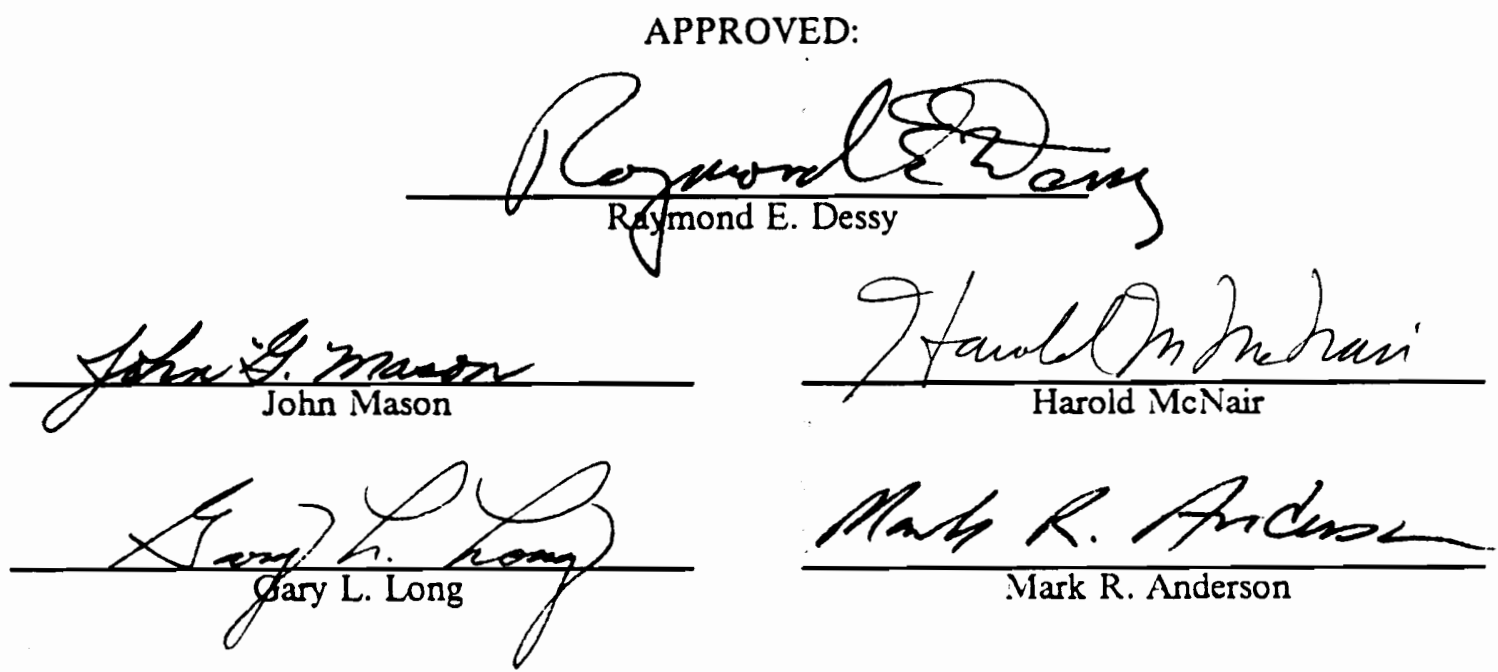

April 18, 1990

Blacksburg, Virginia 


\title{
A Multi-Mode Spectrometer for Atomic Emission Spectrometry \\ by
}

\author{
Mark A. Wingerd \\ Raymond E. Dessy \\ Department of Chemistry
}

(ABSTRACT)

A unique spectrometer system, the Multi-Mode Spectrometer (MMS), has been developed. The MMS integrates a scanning Michelson interferometer, a flat-field grating, and a linear photodiode array detector into a single spectrometer system. With these components, the MMS is capable of applying dispersive, interferometric, or combined dispersive/interferometric techniques for enhanced spectrometric flexibility. The effects of source fluctuation and redistributed photon noise can be reduced. In addition, the MMS has unique capabilities in data compression, application of internal standards, and noise spectrum analysis. 


\section{Acknowledgements}

I would like to express my gratitude to the people who gave me help and support through the duration of this research effort. A great number of people contributed to this research in many ways large and small, direct and indirect. It is difficult to acknowledge them all.

First, I would like to express my gratitude to several people for specific assistance in this research. For support and encouragement early in the project, I would like to thank Dr. Berwyn Jones of the U.S. Geological survey for donation of the polychromator, and Dr. Charles Anderson and Nicolet Instrument Corporation for loan of the interferometer. For enormous technical support and assistance through the middle of the project, I would like to thank Mr. Dale Schutt of the Physics Electronics Shop, VPI\&SU, for much valuable advice on the electronics of the MMS, and Mr. Fred Blair of the Physics Machine Shop, VPI\&SU, for much valuable assistance and advice on mechanical aspects of the MMS. For sharing their expertise and knowledge through the final stages of the project, I would like to thank Dr. J. W. Brault of the National Solar Observatory, for numerous discussions on the intricacies of Fourier transform spectrometry, and Dr. G. M. Hieftje of the Chemistry Department of Indiana University, for numerous discussions on atomic spectrometry and noise.

Next, I would like to express my gratitude to my fellow graduate students of the Laboratory Automation and Instrument Design Group in the Chemistry Department of VPI\&SU. They are 
a truly unique group of talented people, and all of them continuously contributed to the emotional and technical support network of the laboratory. From a technical perspective, I would like to thank Ian Chapple, for much patient assistance in Forth programming, graphics software, and general programming consultation. From a personal perspective, I thank all of the group members; they are all lifetime friends. First among them are Ian Chapple, W. Stephen Conder, Steve Choquette, and Glen Wollenberg.

I would like to thank my research advisor, Dr. Ray Dessy, and his wife, Lee Dessy, for bringing together and contributing to such a diverse group of interesting and talented people.

Within the Chemistry Department, I would like to thank Dr. Gary Long and his group for assistance with the microwave plasma. Also within the department, I would like to thank Dr. John Mason for being a beacon and model for scholarship and ethics.

I would like to thank my family for continued emotional and financial support, and much patience, through my tenure as a doctoral student.

And most of all, I would like to thank my dear and lovely wife, Dr. Diane M. Horm-Wingerd. Without her constant love and devotion, this research would have been extremely difficult. Without her financial support, this research would have been impossible. 


\section{Table of Contents}

OVERVIEW OF THE MULTI-MODE SPECTROMETER $\ldots \ldots \ldots \ldots \ldots \ldots \ldots \ldots 1$

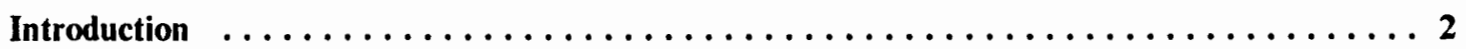

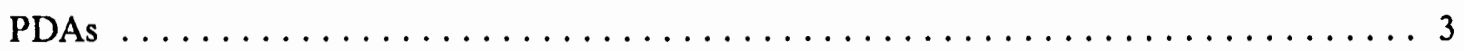

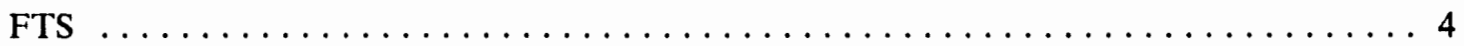

The Multiplex Advantage and Disadvantage in FT-AES $\ldots \ldots \ldots \ldots \ldots \ldots \ldots$

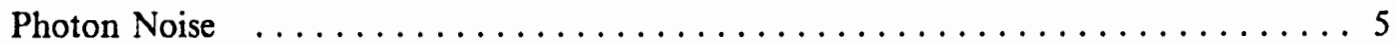

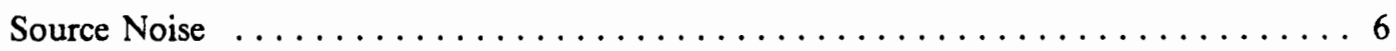

The MMS Modes of Operation $\ldots \ldots \ldots \ldots \ldots \ldots \ldots \ldots \ldots \ldots \ldots \ldots$

The Dispersive Mode $\ldots \ldots \ldots \ldots \ldots \ldots \ldots \ldots \ldots \ldots \ldots \ldots$

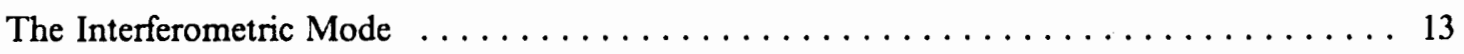

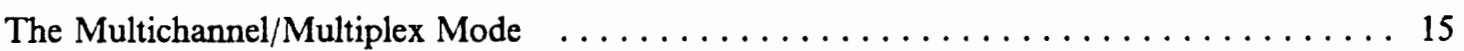

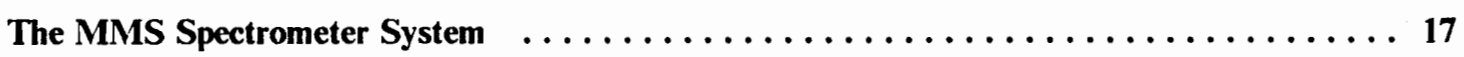

The Optical Plan and Static Design Considerations $\ldots \ldots \ldots \ldots \ldots \ldots \ldots \ldots \ldots$

Dynamic Design Considerations $\ldots \ldots \ldots \ldots \ldots \ldots \ldots \ldots \ldots \ldots \ldots \ldots \ldots \ldots \ldots \ldots$

Table of Contents $\quad v$ 


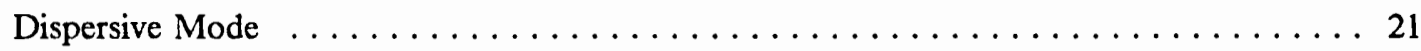

Interferometric and Multichannel/Multiplex Modes $\ldots \ldots \ldots \ldots \ldots \ldots \ldots \ldots . \ldots \ldots$

The MMS Instrument Control and Data Acquisition System $\ldots \ldots \ldots \ldots \ldots \ldots 23$

Theory: Interpretation of MMS Spectra $\ldots \ldots \ldots \ldots \ldots \ldots \ldots \ldots \ldots \ldots \ldots \ldots$

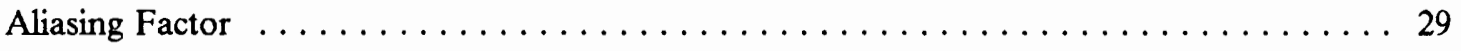

Resolving Power, Resolution, and Spectral Bandwidth $\ldots \ldots \ldots \ldots \ldots \ldots \ldots \ldots$

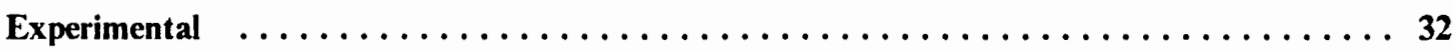

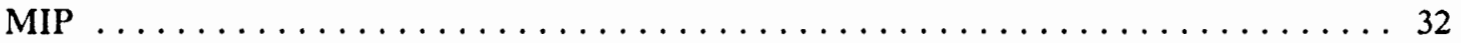

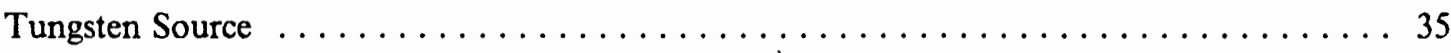

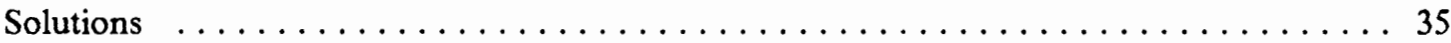

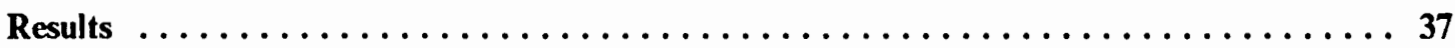

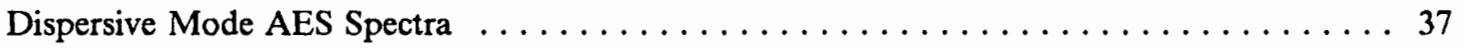

Interferometric Mode White-Light Data $\ldots \ldots \ldots \ldots \ldots \ldots \ldots \ldots \ldots \ldots \ldots$

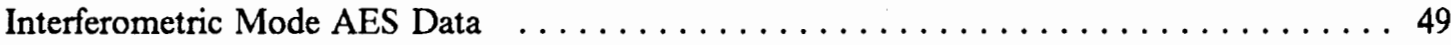

Higher Resolution Interferometric AES Spectra $\ldots \ldots \ldots \ldots \ldots \ldots \ldots \ldots \ldots$

Multichannel/Multiplex Mode AES Data $\ldots \ldots \ldots \ldots \ldots \ldots \ldots \ldots \ldots \ldots . \ldots \ldots$

Spectrometric Performance Summary $\ldots \ldots \ldots \ldots \ldots \ldots \ldots \ldots \ldots \ldots \ldots . \ldots \ldots$

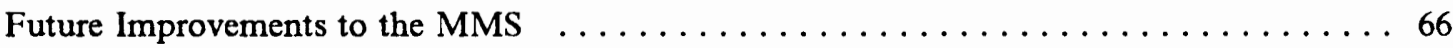

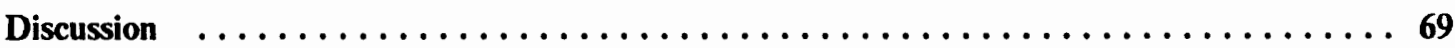

The Throughput Advantage and Limiting Noises in AES $\ldots \ldots \ldots \ldots \ldots \ldots \ldots$

Throughput and the Throughput Advantage of the MMS $\ldots \ldots \ldots \ldots \ldots \ldots \ldots \ldots$

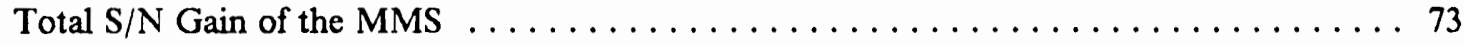

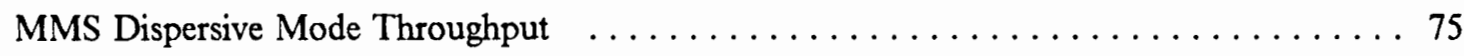

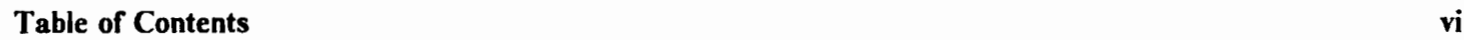




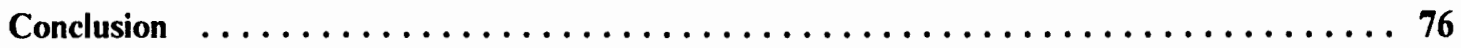

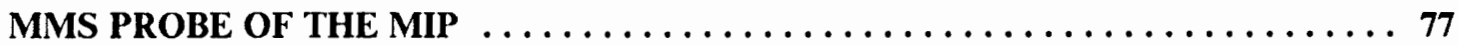

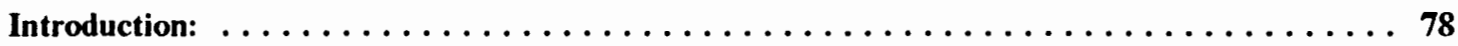

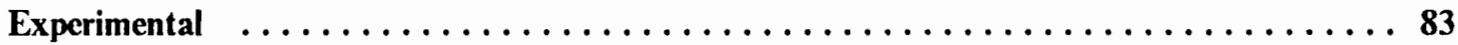

Results and Discussion: $\ldots \ldots \ldots \ldots \ldots \ldots \ldots \ldots \ldots \ldots \ldots \ldots \ldots \ldots \ldots \ldots$

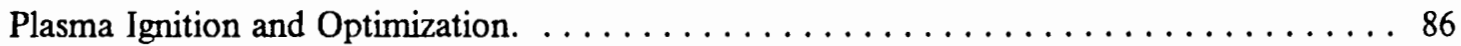

Side-on versus End-on Optical Throughput. $\ldots \ldots \ldots \ldots \ldots \ldots \ldots \ldots \ldots \ldots \ldots$

Side-on Emission Zone Dimensions. $\ldots \ldots \ldots \ldots \ldots \ldots \ldots \ldots \ldots \ldots \ldots$

Basic Plasma Emissions, Side-on Observation $\ldots \ldots \ldots \ldots \ldots \ldots \ldots \ldots \ldots$

Nebulizer Equilibration Time. $\ldots \ldots \ldots \ldots \ldots \ldots \ldots \ldots \ldots \ldots \ldots \ldots \ldots$

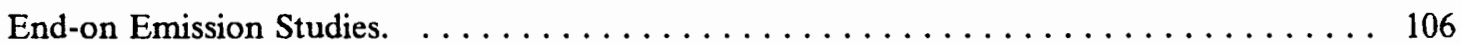

Interferometric Mode: End-on versus Side-on Observation: $\ldots \ldots \ldots \ldots \ldots \ldots \ldots \ldots 117$

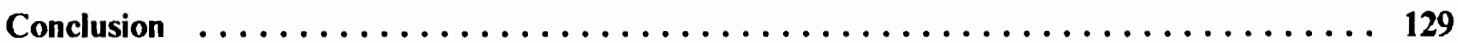

THEORETICAL DEVELOPMENT $\ldots \ldots \ldots \ldots \ldots \ldots \ldots \ldots \ldots \ldots \ldots \ldots$

Theory of Fourier Transform Spectrometry $\ldots \ldots \ldots \ldots \ldots \ldots \ldots \ldots \ldots \ldots \ldots$

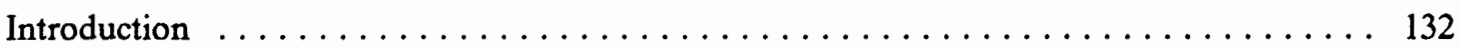

Overview and Advantages of Fourier Transform Spectrometry $\ldots \ldots \ldots \ldots \ldots \ldots \ldots$

Mathematical Foundations: The Fourier Transform $\ldots \ldots \ldots \ldots \ldots \ldots \ldots \ldots \ldots$

The Fourier Integral $\ldots \ldots \ldots \ldots \ldots \ldots \ldots \ldots \ldots \ldots \ldots \ldots \ldots \ldots \ldots \ldots$

The Real and Imaginary Components of the Spectrum $\ldots \ldots \ldots \ldots \ldots \ldots \ldots$

Amplitude and Phase Components of the Spectrum $\ldots \ldots \ldots \ldots \ldots \ldots \ldots$

Table of Contents vii 
Properties of the Fourier Transform $\ldots \ldots \ldots \ldots \ldots \ldots \ldots \ldots \ldots \ldots \ldots$

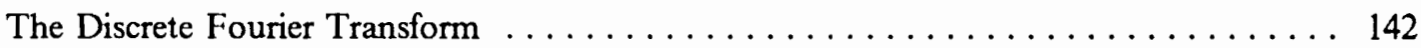

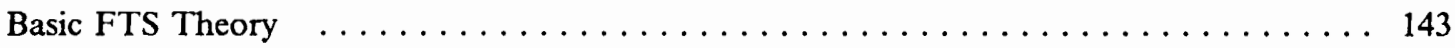

The Ideal Michelson Interferometer and Monochromatic Light $\ldots \ldots \ldots \ldots \ldots \ldots$

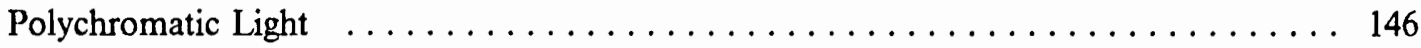

The Michelson Interferometer as a Frequency Shifter $\ldots \ldots \ldots \ldots \ldots \ldots \ldots$

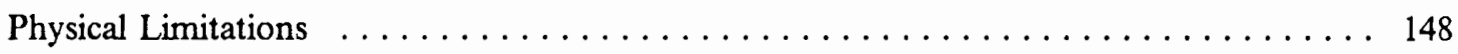

Real Optical Components $\ldots \ldots \ldots \ldots \ldots \ldots \ldots \ldots \ldots \ldots \ldots \ldots \ldots \ldots \ldots$

Finite Path Difference and Resolution $\ldots \ldots \ldots \ldots \ldots \ldots \ldots \ldots \ldots \ldots \ldots$

Finite Source and Detector Apertures $\ldots \ldots \ldots \ldots \ldots \ldots \ldots \ldots \ldots \ldots \ldots \ldots$

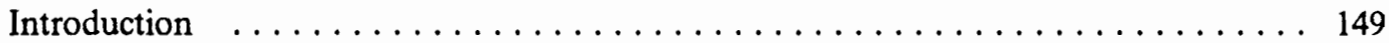

Haidinger Fringes: The Basic Phenomenon $\ldots \ldots \ldots \ldots \ldots \ldots \ldots \ldots 1$

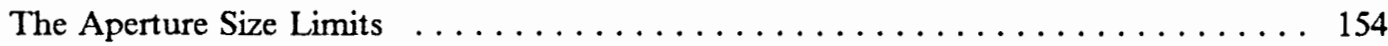

Instrumental Consequences of Finite Apertures $\ldots \ldots \ldots \ldots \ldots \ldots \ldots \ldots$

Interaction of Haidinger fringes and the MMS Optical System $\ldots \ldots \ldots \ldots \ldots 156$

Complementary Fringes: Fizeau Fringes and Haidinger Fringes $\ldots \ldots \ldots \ldots \ldots \ldots$

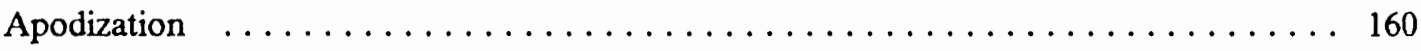

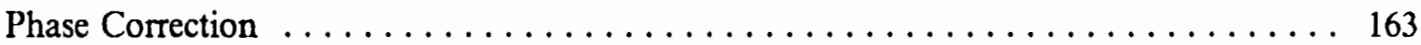

Mirror Scanning: One-sided vs. Two-sided Interferograms . . . . . . . . . . . 164

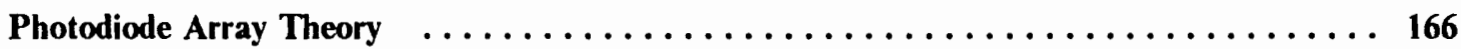

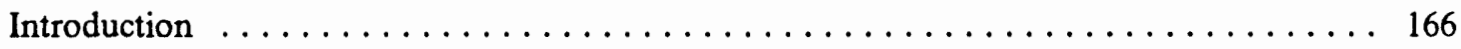

Criteria for a Solid State Detection System $\ldots \ldots \ldots \ldots \ldots \ldots \ldots \ldots \ldots \ldots \ldots$

Description of Photodiode Array and the Spatial Response Function $\ldots \ldots \ldots \ldots \ldots$

The Spatial Response Function $\ldots \ldots \ldots \ldots \ldots \ldots \ldots \ldots \ldots \ldots \ldots \ldots \ldots$

The Spectral Response Function $\ldots \ldots \ldots \ldots \ldots \ldots \ldots \ldots \ldots \ldots \ldots \ldots \ldots$

The Temporal Response Function $\ldots \ldots \ldots \ldots \ldots \ldots \ldots \ldots \ldots \ldots \ldots \ldots \ldots$

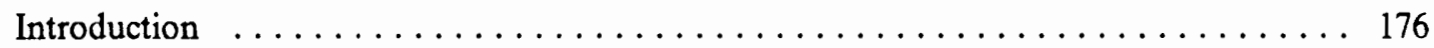

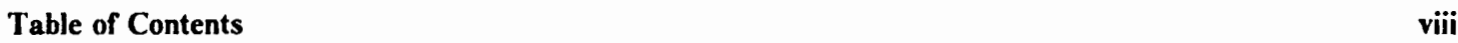


The PDA Transfer Function: The Derivation $\ldots \ldots \ldots \ldots \ldots \ldots \ldots \ldots \ldots$

The Amplitude Response $\ldots \ldots \ldots \ldots \ldots \ldots \ldots \ldots \ldots \ldots \ldots . \ldots \ldots$

The Phase Response $\ldots \ldots \ldots \ldots \ldots \ldots \ldots \ldots \ldots \ldots \ldots \ldots \ldots$

Predicting PDA Frequency Response to General Inputs $\ldots \ldots \ldots \ldots \ldots \ldots \ldots \ldots$

Frequency Response in MMS Design and Mirror/PDA Coordination $\ldots \ldots \ldots \ldots \ldots 2$

Interferometric Mode Response $\ldots \ldots \ldots \ldots \ldots \ldots \ldots \ldots \ldots \ldots \ldots \ldots \ldots \ldots \ldots$

Dispersive Mode Response $\ldots \ldots \ldots \ldots \ldots \ldots \ldots \ldots \ldots \ldots \ldots \ldots \ldots$

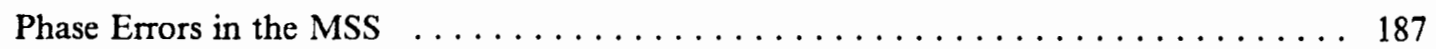

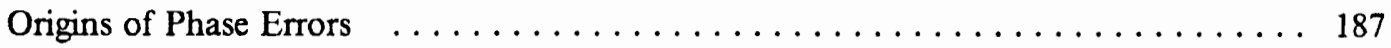

Partial Compensation of Phase Errors $\ldots \ldots \ldots \ldots \ldots \ldots \ldots \ldots \ldots \ldots \ldots$

Noise and Errors within the PDA $\ldots \ldots \ldots \ldots \ldots \ldots \ldots \ldots \ldots \ldots \ldots \ldots$

Readout Noise and Video Line Capacitance $\ldots \ldots \ldots \ldots \ldots \ldots \ldots \ldots \ldots \ldots$

Dark Current and Dark Current Noise $\ldots \ldots \ldots \ldots \ldots \ldots \ldots \ldots \ldots \ldots$

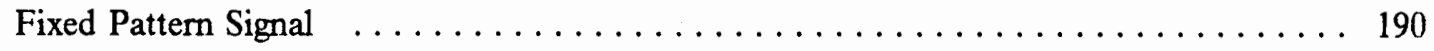

Bibliography $\ldots \ldots \ldots \ldots \ldots \ldots \ldots \ldots \ldots \ldots \ldots \ldots \ldots \ldots \ldots \ldots \ldots \ldots \ldots$

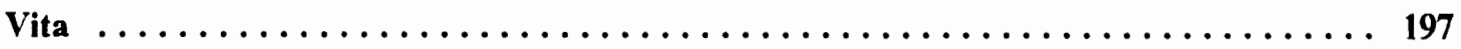

$\begin{array}{ll}\text { Table of Contents } & \text { ix }\end{array}$ 


\section{List of Illustrations}

Figure 1. Information space of the MMS, Dispersive mode $\ldots \ldots \ldots \ldots \ldots \ldots$

Figure 2. Information space of the MMS, Interferometric mode $\ldots \ldots \ldots \ldots \ldots \ldots$

Figure 3. Information space of the MMS, Multichannel/multiplex mode $\ldots \ldots \ldots \ldots \ldots 1$

Figure 4. The effects of correlated source fluctuations on line envelopes $\ldots \ldots \ldots \ldots 12$

Figure 5. Schematic of the MMS optical plan $\ldots \ldots \ldots \ldots \ldots \ldots \ldots \ldots$

Figure 6. Block diagram of the MMS computer and the electronics system $\ldots \ldots \ldots 26$

Figure 7. Cross-section of the MIP apparatus $\ldots \ldots \ldots \ldots \ldots \ldots \ldots \ldots \ldots \ldots \ldots$

Figure 8. Dispersive mode, blank spectrum of the MIP $\ldots \ldots \ldots \ldots \ldots \ldots$

Figure 9. Dispersive mode, sample spectrum of the MIP $\ldots \ldots \ldots \ldots \ldots \ldots \ldots$

Figure 10. Dispersive mode, blank-corrected spectrum of the MIP $\ldots \ldots \ldots \ldots \ldots \ldots$

Figure 11. Interferometric mode, interferogram of the tungsten source . . . . . . . . 44

Figure 12. Interferometric mode, amplitude spectrum of the tungsten source $\ldots \ldots \ldots \ldots$

Figure 13. Interferometric mode, amplitude spectrum of the tungsten source $\ldots \ldots \ldots 46$

Figure 14. Interferometric mode, amplitude spectra of the tungsten source $\ldots \ldots \ldots \ldots 47$

Figure 15. Interferometric mode, AES interferogram $\ldots \ldots \ldots \ldots \ldots \ldots \ldots \ldots$

Figure 16. Interferometric mode, AES spectrum $\ldots \ldots \ldots \ldots \ldots \ldots \ldots \ldots \ldots \ldots \ldots \ldots$

Figure 17. Interferometric mode, AES spectrum $\ldots \ldots \ldots \ldots \ldots \ldots \ldots \ldots \ldots$

Figure 18. Interferometric mode, AES $\log$ spectrum $\ldots \ldots \ldots \ldots \ldots \ldots \ldots$

Figure 19. Interferometric mode, AES spectrum $\ldots \ldots \ldots \ldots \ldots \ldots \ldots \ldots \ldots$

Figure 20. Multichannel/multiplex mode, Na spectrum, full bandwidth spectrum $\ldots \ldots 60$

Figure 21. Multichannel/multiplex mode, $\mathrm{Na}$ spectrum, $400 \mathrm{~cm}^{-1}$ at the $\mathrm{Na}$ lines $\ldots \ldots 61$ 
Figure 22. Multichannel/multiplex mode, $\mathrm{K}$ spectrum, full bandwidth spectrum $\ldots \ldots \ldots 62$

Figure 23. Multichannel/multiplex mode, $\mathrm{K}$ spectrum, $400 \mathrm{~cm}^{-1}$ at the $\mathrm{K}$ lines $\ldots \ldots 63$

Figure 24. Cross-section of the MIP apparatus $\ldots \ldots \ldots \ldots \ldots \ldots \ldots \ldots \ldots \ldots$

Figure 25. Close-ups of the MIP torch emission zones $\ldots \ldots \ldots \ldots \ldots \ldots \ldots$

Figure 26. Optical schematics for side-on and end-on observation geometries $\ldots \ldots \ldots 8$

Figure 27. Dispersive mode, survey spectrum: Ar $\ldots \ldots \ldots \ldots \ldots \ldots \ldots \ldots \ldots \ldots$

Figure 28. Dispersive mode, survey spectrum: $\mathrm{Ar}$ and $\mathrm{H}_{2} \mathrm{O} \ldots \ldots \ldots \ldots \ldots$

Figure 29. Dispersive mode, survey spectrum: $\mathrm{Ar}, \mathrm{H}_{2} \mathrm{O}$, and $\mathrm{Na}(500 \mu \mathrm{g} / \mathrm{mL}) \ldots \ldots \ldots 9$

Figure 30. Dispersive mode, survey spectrum: $\mathrm{Ar}, \mathrm{H}_{2} \mathrm{O}$, and $\mathrm{K}(500 \mu \mathrm{g} / \mathrm{mL}) \ldots \ldots \ldots .95$

Figure 31. Dispersive mode, survey spectrum: $\mathrm{Ar}, \mathrm{H}_{2} \mathrm{O}, \mathrm{Na}(500 \mu \mathrm{g} / \mathrm{mL})$, and $\mathrm{K}(500 \mu \mathrm{g} / \mathrm{mL}) 96$

Figure 32. Dispersive mode, survey spectrum: $\mathrm{Ar}, \mathrm{H}_{2} \mathrm{O}$, and $\mathrm{Na}(100 \mu \mathrm{g} / \mathrm{mL}) \ldots \ldots . .97$

Figure 33. Dispersive mode, difference spectrum: $\mathrm{H}$ and $\mathrm{O} \ldots \ldots \ldots \ldots \ldots \ldots$

Figure 34. Dispersive mode, difference spectrum: $\mathrm{Na}, 500 \mu \mathrm{g} / \mathrm{mL} \ldots \ldots \ldots \ldots \ldots \ldots$

Figure 35. Dispersive mode, difference spectrum: $\mathrm{K}, 500 \mu \mathrm{g} / \mathrm{mL} \ldots \ldots \ldots \ldots \ldots$

Figure 36. Dispersive mode, difference spectrum: $\mathrm{Na}, 500 \mu \mathrm{g} / \mathrm{mL}$, and $\mathrm{K}, 500 \mu \mathrm{g} / \mathrm{mL} \ldots 104$

Figure 37. Dispersive mode, difference spectrum: $\mathrm{Na}, 100 \mu \mathrm{g} / \mathrm{mL} \ldots \ldots \ldots \ldots \ldots$

Figure 38. Dispersive mode, time-series spectra of the nebulizer equilibration time $\ldots \ldots 107$

Figure 39. Dispersive mode, end-on Ar spectrum, observation plane $1 \ldots \ldots \ldots \ldots \ldots$

Figure 40. Dispersive mode, end-on Na spectrum, observation plane $2 \ldots \ldots \ldots \ldots 11$

Figure 41. Dispersive mode, side-on Na spectrum, observation plane $1 \ldots \ldots \ldots \ldots \ldots 112$

Figure 42. Dispersive mode, side-on $\mathrm{H}_{2} \mathrm{O}$ spectrum, observation plane $1 \ldots \ldots \ldots \ldots$

Figure 43. Projected area of the transfer apertures into the microwave plasma $\ldots \ldots \ldots 119$

Figure 44. Interferometric mode data: Side-on interferogram $\ldots \ldots \ldots \ldots \ldots \ldots \ldots$

Figure 45. Interferometric mode data: End-on interferogram $\ldots \ldots \ldots \ldots \ldots \ldots \ldots \ldots$

Figure 46. Interferometric mode: Side-on amplitude spectrum, full bandwidth $\ldots \ldots \ldots 122$

Figure 47. Interferometric mode: End-on amplitude spectrum, full bandwidth $\ldots \ldots \ldots 123$

Figure 48. Interferometric mode: Side-on amplitude spectrum, $400 \mathrm{~cm}^{-1}$ at the $\mathrm{Na}$ doublet 124

Figure 49. Interferometric mode: End-on amplitude spectrum, $400 \mathrm{~cm}^{-1}$ at the $\mathrm{Na}$ doublet 125 
Figure 50. A hypothetical data set and its Fourier transform $\ldots \ldots \ldots \ldots \ldots \ldots$

Figure 51 . The phase and amplitude spectra for a hypothetical data set $\ldots \ldots \ldots \ldots$

Figure 52. The Real, Imaginary, Amplitude, and Phase values for a single frequency . . . 139

Figure 53. The Real, Imaginary, Amplitude, and Phase representation for an entire spectrum 140

Figure 54. Schematic of a Michelson interferometer $\ldots \ldots \ldots \ldots \ldots \ldots \ldots \ldots$

Figure 55. Interferometer output from a monochromatic point source $\ldots \ldots \ldots \ldots \ldots$

Figure 56. Maximum path difference and resolution $\ldots \ldots \ldots \ldots \ldots \ldots \ldots \ldots$

Figure 57 . Haidinger fringes: the spatial intensity profile at the detector plane $\ldots \ldots \ldots 152$

Figure 58. The angle of propagation the interferometer and Haidinger fringes $\ldots \ldots \ldots 153$

Figure 59. Haidinger fringes and interaction with the MMS apertures $\ldots \ldots \ldots \ldots \ldots 157$

Figure 60. Haidinger and Fizeau fringes: Complementary fringes within the interferometer . 159

Figure 61 . Common apodization functions used in FTS $\ldots \ldots \ldots \ldots \ldots \ldots \ldots$

Figure 62 . Common apodization functions, continued $\ldots \ldots \ldots \ldots \ldots \ldots \ldots \ldots$

Figure 63. The PDA sensor geometry and the spatial response function $\ldots \ldots \ldots \ldots 171$

Figure 64 . The equivalent circuit of the RL-0512S photodiode array $\ldots \ldots \ldots \ldots \ldots 172$

Figure 65. The PDA output waveform $\ldots \ldots \ldots \ldots \ldots \ldots \ldots \ldots \ldots \ldots \ldots$

Figure 66. The spectral response and quantum efficiency of the S-series photodiode array .175

Figure 67. The PDA time sampling window and the normalized frequency response $\ldots \ldots 178$

Figure 68 . Various formats for the PDA frequency response $\ldots \ldots \ldots \ldots \ldots \ldots \ldots 1$

Figure 69. Expanded view of the $\log (y)$ vs. $\log (\operatorname{sinc}(x))$ PDA response $\ldots \ldots \ldots \ldots \ldots 183$

Figure 70 . PDA response: First lobe of the transfer function $\ldots \ldots \ldots \ldots \ldots \ldots \ldots$ 


\section{List of Tables}

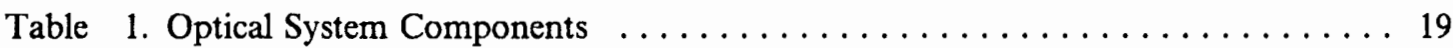

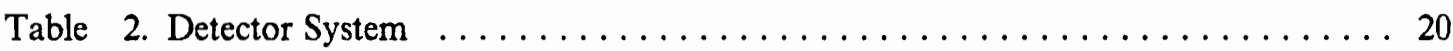

Table 3. Interferometric and Multichannel/Multiplex Modes: Options and Specifications . . 24

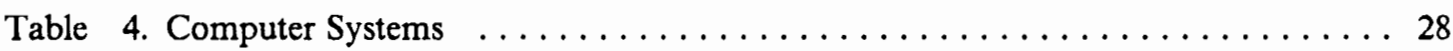

Table 5. Equipment for the Spectral Sources $\ldots \ldots \ldots \ldots \ldots \ldots \ldots \ldots \ldots$

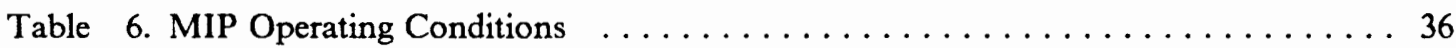

Table 7. Reference Data for MIP Line Assignments $\ldots \ldots \ldots \ldots \ldots \ldots \ldots \ldots$

Table 8. Common MMS Operating Parameters for Figures 8, 9, and $10 \ldots \ldots \ldots \ldots 4$

Table 9. Operating Parameters for Figures 11, 12, 13, and $14 \ldots \ldots \ldots \ldots$

Table 10. Operating Parameters for Figures 15, 16, 17, and $18 \ldots \ldots \ldots \ldots \ldots \ldots$

Table 11. Operating Parameters for Figure $19 \ldots \ldots \ldots \ldots \ldots \ldots \ldots \ldots \ldots \ldots \ldots \ldots \ldots \ldots \ldots$

Table 12. Operating Parameters for Figures 20 and $21 \ldots \ldots \ldots \ldots \ldots \ldots$

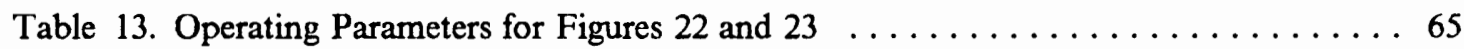

Table 14. MMS Determination of MIP Line Parameters $\ldots \ldots \ldots \ldots \ldots \ldots \ldots$

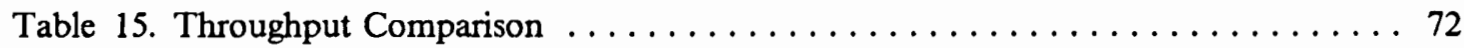

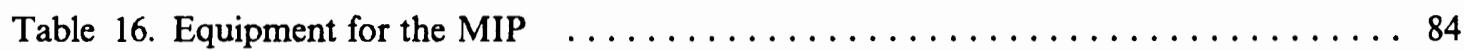

Table 17. MIP Operating Conditions $\ldots \ldots \ldots \ldots \ldots \ldots \ldots \ldots \ldots \ldots \ldots \ldots \ldots \ldots \ldots \ldots \ldots \ldots$

Table 18. Experimental Parameters for the Emission Survey Spectra $\ldots \ldots \ldots \ldots \ldots \ldots 91$

Table 19. Species Introduced into the MIP for the Survey Spectra $\ldots \ldots \ldots \ldots \ldots \ldots$

Table 20. Emission Species in the Survey Difference Spectrum . . . . . . . . . . . 99

Table 21. Experimental Parameters for the Nebulizer Equilibration Time Spectra . . . . . 108 
Table 22. Experimental Parameters for the End-on Emission Zone Spectra . . . . . . . 114

Table 23. End-on Emission Summary: Ar Core vs. Na Annulus $\ldots \ldots \ldots \ldots \ldots \ldots \ldots$

Table 24. Experimental Parameters, Interferometric Mode Comparison of End-on vs. Side-on

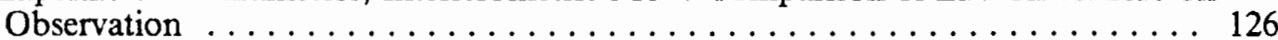

Table 25. Summary: Interferometric Study of Observation Geometry, Signal and Noise Amplitudes ................................ 127

Table 26. Photodiode Array Specifications. . . . . . . . . . . . . . . . . 169 


\section{Preface}

This dissertation was written in three major sections. The first section provides an overview of the purpose and capabilities of the multi-mode spectrometer (MMS). This first section discusses the goals of the MMS development and the trade-offs necessary for this process. The second section shows further application of the MMS to characterizing an atomic emission source. The unique capabilities of the MMS were used to probe spatial, spectral, and temporal characteristics of the microwave induced plasma. The third section discusses the theories of photodiodes array detectors and Fourier transform spectrometry. A number of critical theoretical developments derived in the course of this research are discussed, in addition to standard theoretical treatments of FTS and array detectors.

This dissertation therefore may be read in a number of orders. To obtain an integrated overview of the MMS, read section one. To obtain a more complete picture of the MMS' capabilities and the emission characteristics of the MIP, read section two. To gain a better grasp of the theory behind the MMS and the additional theoretical developments behind the MMS, read section three. 


\section{Overview of the Multi-Mode Spectrometer}




\section{Introduction}

The purpose of this research was to develop a unique spectrometer system, the Multi-Mode Spectrometer (MMS), and to apply the spectrometer to atomic emission spectrometry (AES). The objective was to combine a photodiode array detector (PDA) and Fourier transform spectrometry (FTS) in a single spectrometer. The goals were to retain the individual advantages of PDAs and FTS, to reduce the individual disadvantages, and to investigate synergistic strengths.

Physically the MMS incorporates a scanning Michelson interferometer, a flat-field grating, and a linear PDA in a single spectrometer system. More significantly, the MMS is capable of multichannel (dispersive), multiplex (interferometric), and multichannel/multiplex modes of operation.

Photodiode arrays (PDAs) and Fourier transform spectrometry (FTS) have been two of the most interesting and promising technologies that have recently been applied to AES. Each of these has distinctive advantages over conventional detection systems when applied to atomic emission spectrometry (AES). Unfortunately, PDAs and FTS also have distinct disadvantages. The individual advantages and disadvantages of FTS and PDAs are discussed below.

The MMS was developed with several major goals in mind. One goal is spectrometric flexibility. With the MMS, either dispersive or interferometric techniques may be selected for a given problem. Spectrometric parameters such as resolution, spectrum readout rate, integration 
time, spectral bandwidth, data acquisition density (aliasing), and apodization are also user selectable. In the multichannel/multiplex mode, different parameters may be specified for each channel. In sum, the performance characteristics of the MMS can be precisely tuned to the demands of a given problem, or even a specific spectral bandwidth.

A second major goal of the MMS is the reduction of redistributed noise effects in multiplex spectrometry. Specifically, the goal is to reduce the redistributed effects of photon and source noise in the interferometric and multichannel/multiplex modes. Reduction of these noises can reduce or eliminate the multiplex disadvantage. ${ }^{1}$

A third major goal is to develop a system with unique capabilities in the following areas: data compression, the application of internal standards, and noise spectrum analysis.

As a means of testing the MMS and demonstrating its capabilities, the MMS has been applied to atomic emission spectrometry. The emission signals from a microwave induced plasma (MIP) has been observed. It should be emphasized that the MMS is a prototype instrument and has not been fully optimized.

\section{$P D A s$}

Photodiode arrays, ${ }^{2}$ as well as other types of solid-state imaging detectors, such as charge-coupled devices (CCDs) and charge-injection devices (CIDs), ${ }^{3,4}$ have been applied to spectrochemical analysis for a number of years. Horlick and co-workers, ${ }^{5,6}$ Talmi and Simpson, ${ }^{7}$ and Grabau and Talmi ${ }^{8}$ have characterized PDAs and PDA systems as spectrochemical detectors. Denton and co-workers have similarly characterized CCDs and CIDs.,10 Recently, several novel optical designs have been developed which efficiently utilize the advantages of solid state imaging detectors. ${ }^{11,12}$

PDA-based spectrometers offer the significant advantages of simultaneous observation of signal and background over a wide spectral range, rapid data acquisition, and improvements in the 
S/N. In spite of this, PDAs have not found great acceptance in AES. Primarily, this has been because the number of observed optical channels, usually $1,024^{6}$ to $4,096,{ }^{13}$ is much less than the resolving power of $20,000{ }^{14}$ to $50,000^{8}$ used for routine analysis and is considerably less than the 150,000 is to $>500,000^{16}$ needed for resolving atomic line widths. With array detectors, some compromise between resolution and wavelength coverage is always required. PDAs often have other problems when applied to AES, such as low dynamic range $(<1: 25,000),{ }^{17,6,18}$ high read noise $\left(>1,200 \mathrm{e}^{-}\right),{ }^{19,6}$ inflexible resolution within a particular optical design, ${ }^{8}$ and line-to-pixel registration problems. $^{20}$

\section{FTS}

There has been considerable interest in Fourier transform atomic emission spectrometry (FT-AES) for a number of years. Horlick and co-workers, ${ }^{21-28}$ Faires and co-workers,, ${ }^{29-34}$ and Thorne and co-workers ${ }^{1,16,35-37}$ have been the most active in investigating the application of FTS to AES. Much recent interest has been due to the development of high resolution FT spectrometers designed specifically for the ultraviolet and visible. ${ }^{38-41,36}$ Several excellent papers discussing the fundamentals of FTS and FT-AES have been written by Thorne ${ }^{16}$ and Faires. ${ }^{33}$

FTS offers a number of significant advantages over traditional AES detection systems: a wide and continuous free spectral range, flexible and high resolution, high optical throughput, excellent wavenumber precision and accuracy, simultaneous observation of signal and background, and a simple and predictable instrument line function. 


\section{The Multiplex Advantage and Disadvantage in FT-AES}

Of the conventional FTS advantages, ambiguity exists concerning the multiplex advantage in FT-AES. $1,32,26,28,16$ The full multiplex advantage exists only for detector-noise limited conditions. AES, however, is usually photon- or source-noise limited. In these cases, the multiplex advantage is reduced, possibly turning into a multiplex disadvantage. It is also sample and spectral position dependent.

The multiplex disadvantage in FT-AES is well known ${ }^{42}$ and documented. ${ }^{1,26,32,28}$ It occurs in any type of multiplex spectrometry, either Fourier or Hadamard, ${ }^{43}$ under photon-42-45 or source-noise ${ }^{46}$ limited conditions. In these cases, noise is carried through the encoding process by spectral features and redistributed across the spectrum by the transform process. The specific effect of the redistributed noise depends upon the noise type (photon or source) and the noise distribution (1/f, white, or single-frequency). Relative to dispersive spectra, weak spectral features in FTS are often obscured by the additional redistributed noise background.

\section{Photon Noise}

Photon noise, a white noise, is redistributed uniformly throughout an FTS spectrum, producing a uniform noise background across the spectrum. The magnitude of the redistributed photon noise is proportional to the square root of the integrated intensity of the entire spectrum. Significantly, for photon noise in FTS, the $\mathrm{S} / \mathrm{N}$ is improved in spectral regions with greater than average intensity, and is degraded in regions with less than average intensity. ${ }^{47,45}$ Modern AES plasma sources, particularly the ICP, are highly luminous. An analytical plasma emits thousands of lines, many of them very intense, with lines arising from the analyte, co-analytes, solvent, and plasma gas. A strong background continuum is often present as well. The combined effects of redistributed photon noise and highly luminous sources has two effects in FT-AES. First, the redistributed photon noise from irrelevant features can contribute significantly to the noise at the 
analyte line. ${ }^{1,32,26}$ This is particularly true if the analyte is near the detection limit. Second, the photon noise level is highly sample dependent.

Bandlimiting the spectrum with filters ${ }^{1}$ or a monochromator ${ }^{27}$ has been shown to reduce redistributed photon noise and improve the $\mathrm{S} / \mathrm{N}$ at lines of interest. Spectral bandlimiting can be thought of as improving the $\mathrm{S} / \mathrm{N}$ from two perspectives. First, intense and/or irrelevant lines are rejected, thus eliminating the noise that would be redistributed onto an analytical line. This improves the S/N relative to an unfiltered FT spectrometer. Second, photon noise from inside the filter bandpass is redistributed from inside the region of interest and into the spectral filter cut-off regions. The redistribution of the noise may improve the $\mathrm{S} / \mathrm{N}$ relative to a dispersive spectrometer. Spectral bandlimiting does, however, reduce the flexibility in FTS to randomly access any line over a wide spectral range.

\section{Source Noise}

Source noise is a multiplicative noise. In FTS, a multiplicative noise is side-banded around each line. ${ }^{48}$ In AES, source noise generally has a $1 / f$ distribution..$^{49}$ The combination of side-banding and the $1 / \mathrm{f}$ noise distribution cause each line to be broadened. ${ }^{50}$ The broadening is different for each line, and reflects the physical processes leading to the production of a line. ${ }^{50}$ The effects tend to be greatest at the line and in the line wings. ${ }^{28}$

Source noise is a particularly severe problem for both interferometric and dispersive spectrometer systems. Because source noise is directly proportional to the intensity of the line, its effects cannot be reduced by increasing sample integration time; ${ }^{51}$ by increasing spectrometer throughput; ${ }^{16}$ or by the use of modulation techniques, such as chopping, use of lock-in amplifiers,,$^{52}$ or use of an interferometer. ${ }^{28}$ Source noise is, however, often positively correlated between analyte lines.53 Because of this correlation, source noise can be reduced or eliminated through the proper use of internal standards. ${ }^{54-56}$ Criteria for the proper use of internal standards 
include the following: simultaneous observation of analyte lines; detection systems with matched optics, detectors, and electronics; ${ }^{54}$ and detection systems with equivalent views of the source. 55 


\section{The MMS Modes of Operation}

The MMS attempts to retain many of the separate advantages of PDAs and FTS, and reduce the disadvantages. The key to this are the three operating modes. The operating modes are summarized in Figures 1 to 4. These Figures show a hypothetical spectrum with analyte lines (a), (b), and (c), plasma lines (p), and a broadband spectral background.

\section{The Dispersive Mode}

The purpose of the dispersive mode is to allow the rapid acquisition of low-resolution, wide-band spectra. Gross spectral information on line position, intensity, and background are readily obtained; see Figure 1. The MMS detects a $300 \mathrm{~nm}$ spectral range over 512 detector elements (pixels). The spectral range for this work was from 500 to $800 \mathrm{~nm}$. Spectra can be obtained at up to $30 \mathrm{spectra} / \mathrm{sec}$, at a resolution of $1.2 \mathrm{~nm}$ (an average resolving power of 500), and over the $300 \mathrm{~nm}$ spectral range. Dispersive mode spectra are useful for low-resolution survey spectra or temporal studies of emission sources. 
$\varangle$

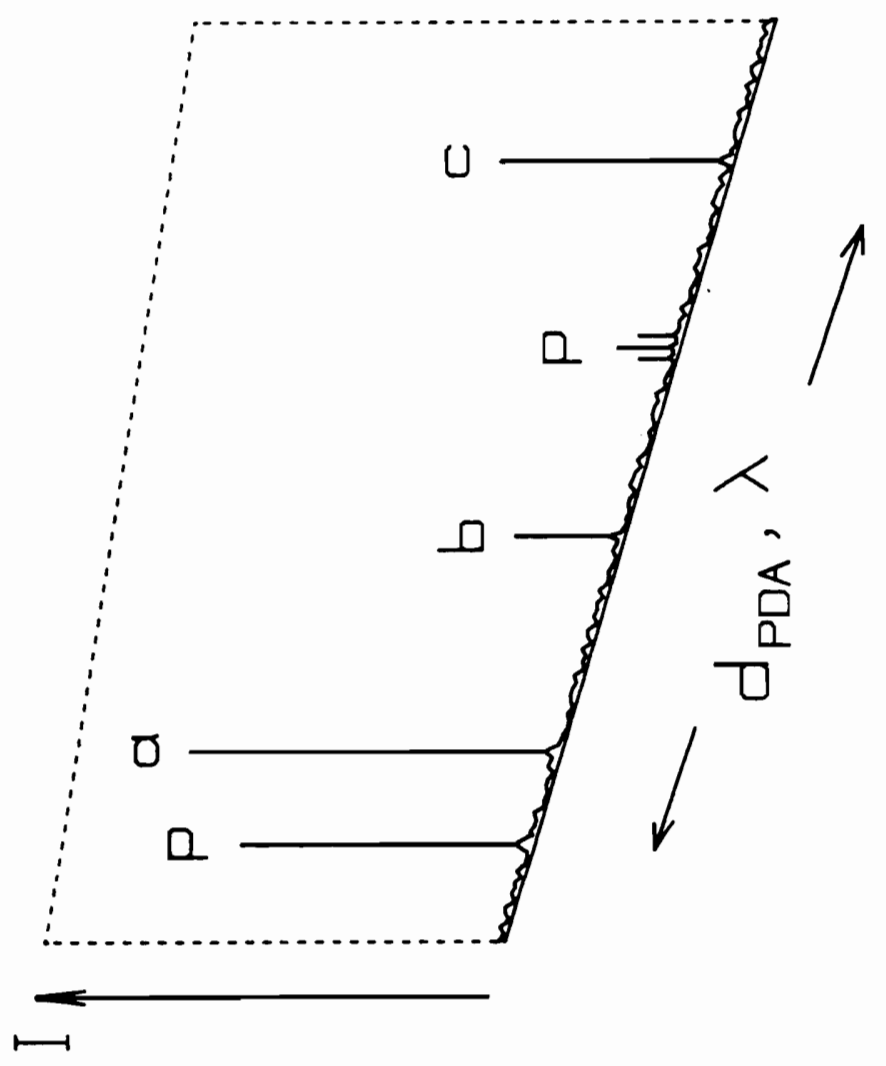

홍

로용

פ

:

可

㐘

$\circ$ 웅

포

号

๑ミ

붕

홍

帘学

흘

ติ

$\sum^{\infty}$

$<$

害立

웧

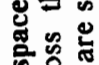

둥

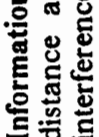

㐫 
$\varpi$

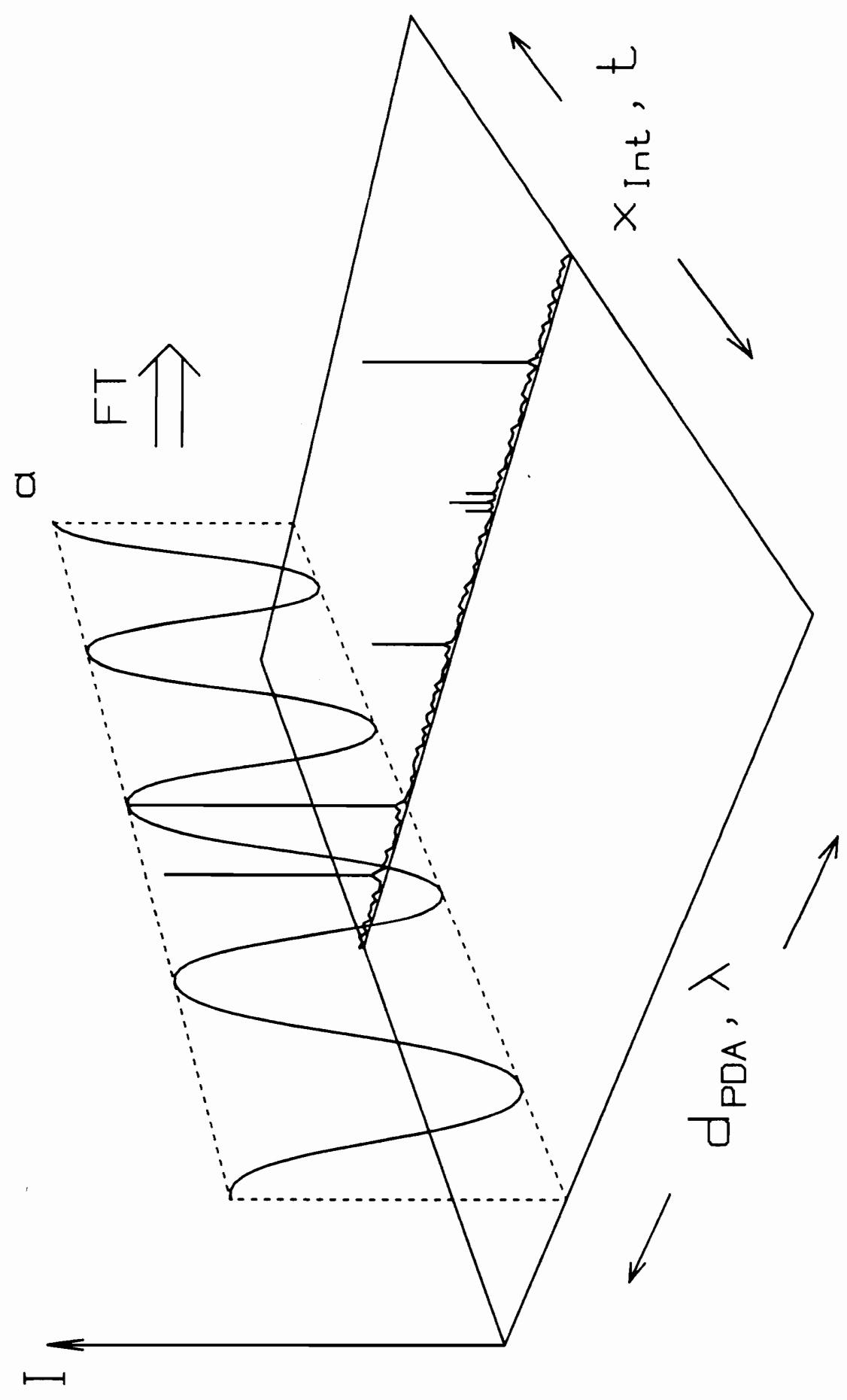

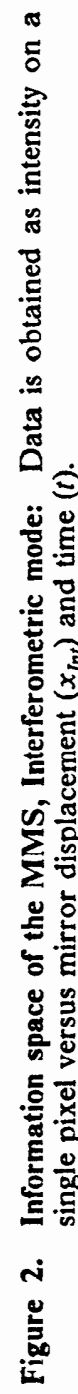


$\sqcup$

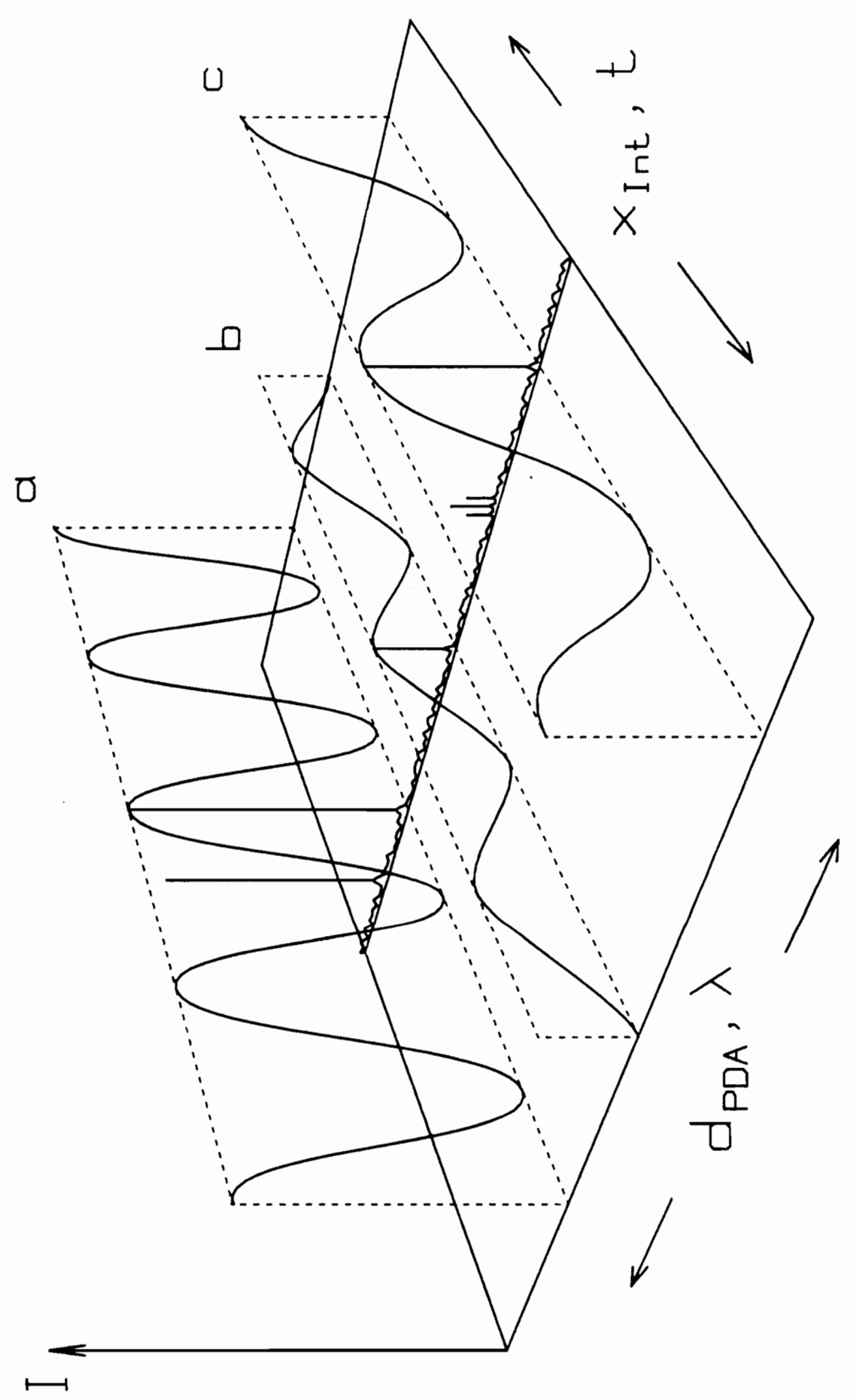

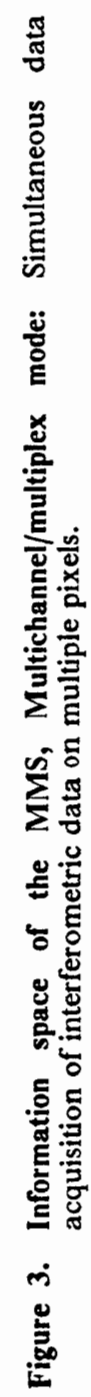


$\square$

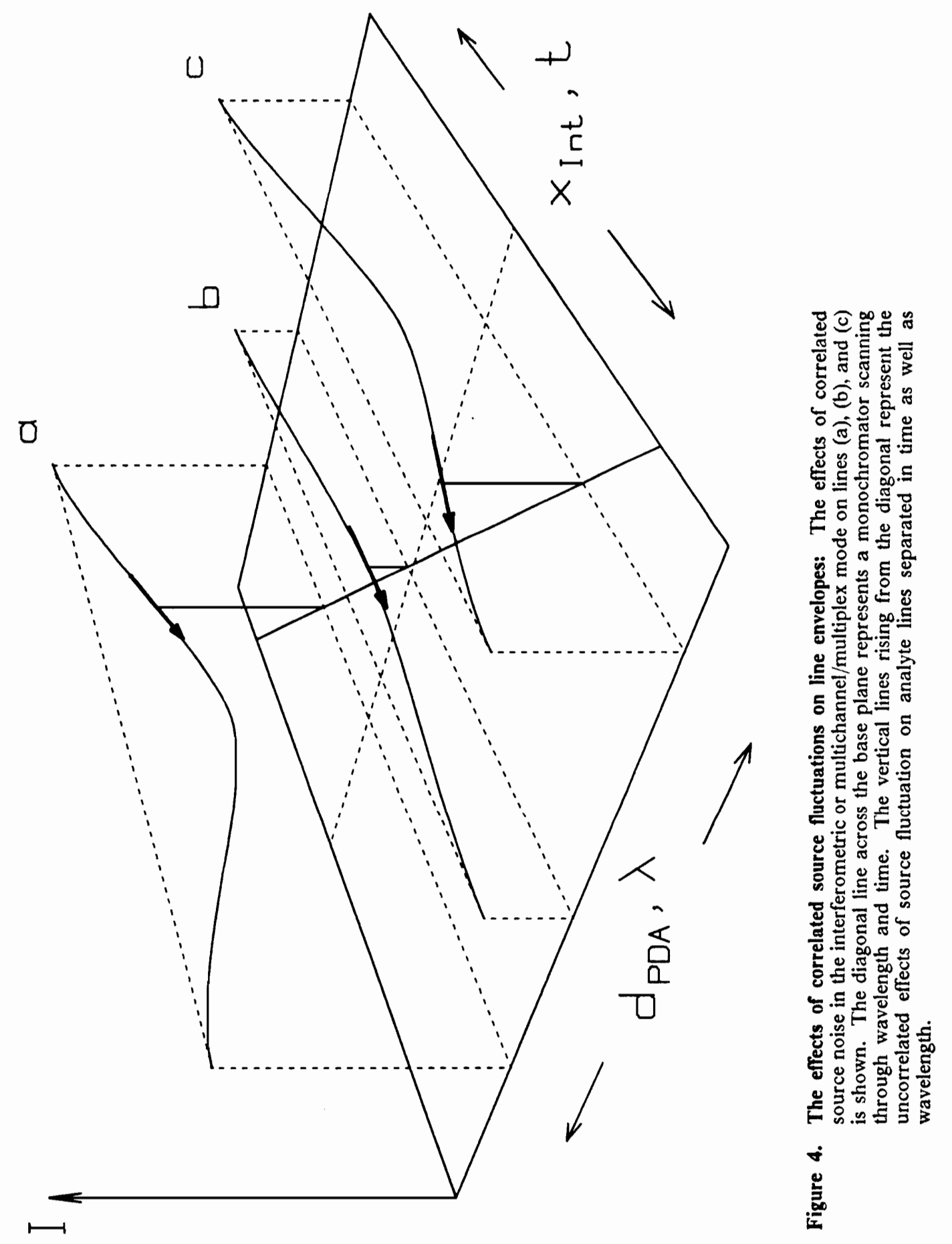


In the dispersive mode the MMS functions exactly as a PDA-based multichannel spectrometer. The grating disperses the light across the array, and the array elements are read out sequentially. Distance across the array, $d_{P D A}$, corresponds to wavelength, $\lambda$. A single data record is intensity versus wavelength. In this mode, the interferometer is disabled as an active optical component (see below). Spectrometric parameters such as integration time and array readout rate are user selectable. Standard multichannel techniques such as variable integration time and on-target integration ${ }^{7}$ are available. Because a holographic grating is used, effects of stray light are reduced.

\section{The Interferometric Mode}

The purposes of the interferometric mode are to provide high resolution spectrometry over a narrow spectral range and to reduce the effects of redistributed photon noise. Operationally, the PDA and polychromator select and isolate the spectral bandwidth of interest. The interferometer is then set in motion, obtaining high resolution FTS data on the selected bandwidth. An interferogram in the MMS is the intensity detected by a single pixel versus interferometer mirror displacement, $x_{I n t}$. The data is Fourier transformed to obtain the spectrum: intensity versus wavenumber. This process is illustrated in Figure 2.

In this mode the MMS functions as a Fourier transform spectrometer with a narrow-bandpass optical filter on the output. The PDA and polychromator function as a flexible optical filtering system. Because the optical bandwidth of the filter is $6 \mathrm{~nm}$ or less, many spurious spectral features are rejected.

The interferometric mode provides a number of direct advantages. Many advantages of FTS such as higher resolution, wavenumber accuracy, and intensity accuracy are available. Flexible spectral filtering by the PDA and polychromator reduces photon noise and the multiplex disadvantage by rejecting unwanted emission lines and spectral background. Comprehensive line 
selection is not compromised, however, because the PDA permits easy and rapid access to any given spectral window. Pixels are selected electronically and can be changed at a rate of $3.2 \mathrm{kHz}$. Resolution is easily changed by selecting the appropriate interferometer mirror displacement. Spectrometric parameters such as resolution, sample interval (alias), and spectral bandwidth selection are user selectable. With the MMS, spectra have been acquired in the interferometric mode at a resolution of $0.5 \mathrm{~cm}^{-1}$ and a resolving power of 38,000 . Additionally, the effects of scattered light should be extremely low because of the holographic grating and the immunity of FTS to stray light.

The interferometric mode has several dispersive advantages that are provided by the PDA/polychromator subsystem. First, the dynamic range requirement of the detection system is reduced. In a typical interferometer, a single detector must respond linearly to the intense centerburst as well as to the weak modulations at the extremes of the interferogram. In the MMS, the source radiation is dispersed across the array, thereby distributing the intensity of the center burst is over a number of pixels. This substantially reduces the dynamic range requirement for a single detector.

A second dispersive advantage is the MMS's ability to perform controlled aliasing. In a typical interferometer, aliasing is avoided. To prevent unintentional aliasing and the resultant folding and mixing of spectral features from different regions, the sampling interval must be kept sufficiently short. The limit for the interval is given by the equation

$$
\delta x \leq \frac{1}{2 \sigma_{\max }}
$$

where $\delta x$ is the sampling interval in the interferogram in $(\mathrm{cm})$, and $\sigma_{\max }$ is the maximum detectable wavenumber. If the spectral bandwidth can be controlled however, the relationship becomes

$$
\delta x \leq \frac{1}{2\left(\sigma_{\max }-\sigma_{\min }\right)}
$$

where $\left(\sigma_{\max }-\sigma_{\min }\right)$ is the spectral bandwidth of the optical filtering system. In the MMS, the polychromator and PDA provide precise optical filtering; they limit the optical bandwidth to $6 \mathrm{~nm}$ or less and they establish the position of any line to within several nanometers. Because of the 
bandwidth control, the data can be highly aliased without fear of spectral overlaps. Because of the line position data from the array, total line position information is maintained. Data from low resolution dispersive spectra and high resolution interferometric spectra are combined to establish the position of any line unambiguously. Controlled aliasing reduces total system overhead by reducing the number of data points that must be acquired, transformed, and stored. Through use of controlled aliasing, the MMS has obtained interferometric data up to an alias of 256.

A third dispersive advantage is the MMS's capability to perform noise spectrum analyses in the interferometric mode. Noise spectrum analysis is a well recognized tool for noise reduction and instrumentation optimization. ${ }^{57,49,58}$ As shown in Figure 2, interferograms are acquired over time, $t(\mathrm{sec})$, as well as mirror displacement, $x_{I n t}(\mathrm{~cm})$. Fourier transforming the data produces a spectrum that can be interpreted as intensity versus frequency $(\mathrm{Hz})$ as well as intensity versus wavenumbers $\left(\mathrm{cm}^{-1}\right)$. In the MMS, the optical filtering by the PDA and polychromator precisely define the spectral frequencies passed by the optical system. Consequently, frequencies produced by noise and interferences are often separated and easily differentiated from the signal frequencies produced by the interferometer. Specific noises or interferences are often easily identified in this format. In addition, the noise at the spectral line is reduced.

\section{The Multichannel/Multiplex Mode}

The multichannel/multiplex mode is an important extension of the interferometric mode. The purposes of this mode are to provide additional data acquisition flexibility, and to reduce source noise. In the multichannel/multiplex mode, interferograms are acquired simultaneously from several pixels. This process is illustrated in Figure 3 for analyte lines (a), (b), and (c). In this mode, the advantages of the interferometric mode are available for a number of independent channels. Additionally, parameters such as resolution, apodization, and aliasing can be uniquely specified for the individual channels. 
As mentioned above, source noise can be a particularly difficult problem. The effects of source noise are modeled in Figure 4 for analyte lines (a), (b), and (c). The dashed rectangles show the maximum unperturbed intensities for analyte lines. The multiplicative and positively correlated effects of source fluctuation are shown by the solid curved lines. Because the array pixels perform as detectors for equivalent but essentially independent interferometers, the MMS meets a number of the criteria for internal standards: ${ }^{54-56}$ First, data is acquired over the same time interval. Second, the optics, detectors, and electronics of the detection systems are matched. And third, the detection systems view the same region of the source. These factors can reduce the effects of source fluctuations on the signal. For identification of source noises and other interferences, comparison of noise spectra between channels is often useful.

To illustrate the importance of simultaneous detection when applying internal standards, Figure 4 also shows the effects of source fluctuations on a linearly scanned monochromator. The monochromator scans through time as well as wavelength, as indicated by the diagonal line in the base plane. Consequently, emission lines experience different attenuations and rates of change that cannot be correlated between "channels", as shown by the vertical lines and the diagonal arrows. Correction for these effects is impossible with a scanned detection system. 


\section{The MMS Spectrometer System}

\section{The Optical Plan and Static Design Considerations}

Figure 5 shows the scaled optical schematic for the MMS. This Figure summarizes the design aspects that are not dependent upon dynamic factors, the dynamic factors being interferometer mirror movement and detector array scanning. L1 and L2 are the collimating and focusing optics respectively for the interferometer. These lenses are spherical achromats, chosen to minimize chromatic and spherical aberrations. The spectral source of interest is placed at $\mathrm{S}, 120$ $\mathrm{mm}$ in front of L1. The interferometer is immediately behind $\mathrm{L} 1$. The components of the interferometer are: BS, the beamsplitter assembly; M1, the moving mirror; M2, the stationary mirror; WhLt, the white light reference source; and $\mathrm{HeNe}$, the helium-neon tracking laser. A clear aperture of $40 \mathrm{~mm}$ is utilized through $\mathrm{L1}, \mathrm{L} 2$, and the interferometer. The transfer aperture, $\mathrm{Al}$, is placed $120 \mathrm{~mm}$ past $\mathrm{L} 2$. A1 is located at the focal points of both L2 and G, the polychromator grating. The components of the polychromator are: M3, the beam deflection mirror; and $\mathrm{G}$, the low-resolution concave holographic grating. PDA is the photodiode array detector. Table 1 lists further optical components specifications and Table 2 lists the PDA specifications. 


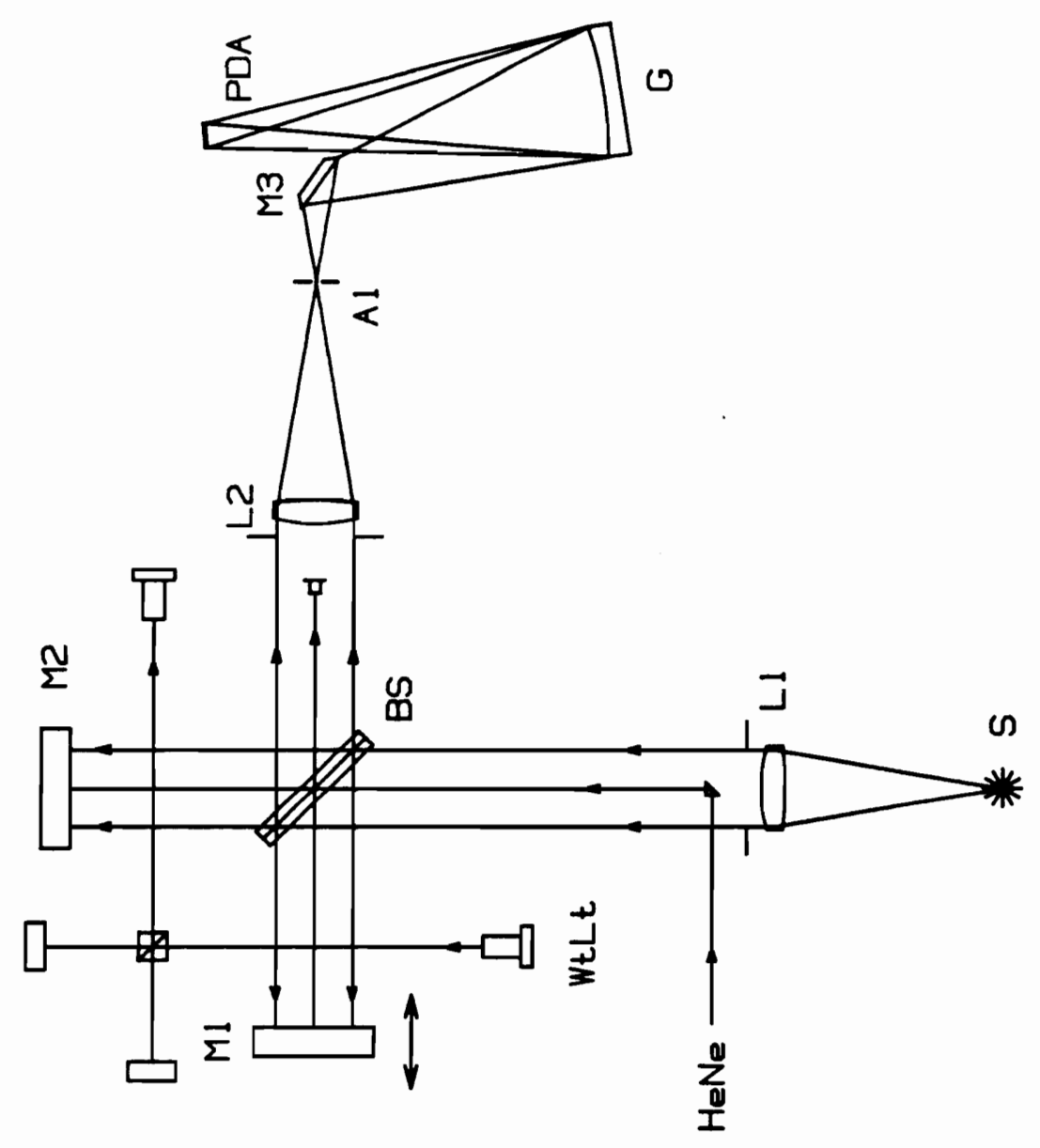


Table 1. Optical System Components

Collimation and focusing lenses:

Manufacturer:

Model:

Type:

Transmission range:

Focal length:

Diameter:

f/\#:

Coating:

Flat field polychromator:

Manufacturer:

Model:

Grating:

Diameter:

Focal length:

f/\#:

Ruling:

Reciprocal linear dispersion:

Slit widths:

Slit heights:

\section{Interferometer system \\ Manufacturer: \\ Interferometer: \\ Model:}

Sample beam,

maximum diameter:

Mirror bearings:

Optical path difference, maximum, double-sided:

Resolution maximum, doubledsided interferogram:

Mirror velocity:

$\mathrm{HeNe}$ base modulation frequency:

Beam splitter:

Model:

Substrate and compensator plate:

Beam splitting material:

Spectral range, nominal:
Melles-Griot, Irvine, CA

01 LAU 145

Spherical achromatic doublet

$340-2500 \mathrm{~nm}$

$120 \mathrm{~mm}$

$43.5 \mathrm{~mm}$

f $/ 2.76$

$\mathrm{MgF}_{2}$

Instruments SA, Edison, NJ

UFS-200

Type III, aberration corrected, flat-field, concave, holographic

$70 \mathrm{~mm}$

$210 \mathrm{~mm}$

$\mathrm{f} / 3$

200 groves $/ \mathrm{mm}$

$24 \mathrm{~nm} / \mathrm{mm}$

$0.050,0.100$, or $0.250 \mathrm{~mm}$

1,3 , or $5 \mathrm{~mm}$

Nicolet Instrument Corp., Madison, WI

7001P, from a 7199A FT-IR with 7199 controller

$45 \mathrm{~mm}$

Parallel dual cylindrical air bearings

$1.037 \mathrm{~cm}$

$0.4823 \mathrm{~cm}^{-1}$

$0.253 \mathrm{~mm} / \mathrm{sec}$

$800 \mathrm{~Hz}$

NIC B7004A

Quartz

proprietary

$25,000-3,300 \mathrm{~cm}^{-1}$

$400-3,000 \mathrm{~nm}$ 
Table 2. Detector System

Array Detector:

Manufacturer:

Type:

Model:

Pixel size:

Total photoactive area:

Operating temperature:

Array Drive Board:

Manufacturer:

Description:

Detector system timing:

Dispersive mode:

Array clock rate:

Pixel output rate:

$A / D$ rate:

Array readout rate, $(\max )$ :

Integration time, (min):

Interferometric and

Multichannel/Multiplex Modes:

Array clock rate:

Pixel readout rate:

Array readout rate:

A/D rate, (max):

Array aperture time, (integration time):
Reticon Corp., Sunnyvale, CA Photodiode array, 512 element self-scanning, linear

RL-0512S

$2.5 \mathrm{~mm}(\mathrm{~h}) \times 25.0 \mu \mathrm{m}(\mathrm{w})$

$2.5 \mathrm{~mm}(\mathrm{~h}) \times 12.8 \mathrm{~mm}(\mathrm{w})$

$25^{\circ} \mathrm{C}$

Reticon Corp., Sunnyvale, CA

RC-1024S evaluation board, lab-modified for switch-selectable internal or external control and clocking.

$62.500 \mathrm{kHz}$

$15.625 \mathrm{kHz}$

$15.625 \mathrm{kHz}$

30 scans/sec

$33 \mathrm{msec}$

$8 \mathrm{MHz}$

$2 \mathrm{MHz}$

$3,200 \mathrm{scans} / \mathrm{sec}$

$3.2 \mathrm{kHz}$

$312.5 \mu \mathrm{sec}$ 
$\mathrm{L} 1$ and L2 were chosen to maintain the $\mathrm{f} / 3$ throughput of the polychromator, and extend it to the entire optical system. The symmetry of L1 and L2 causes a 1:1 imaging of the source onto A1; the polychromator then projects a 1:1 image of Al onto the PDA.

Al is a particularly important component of the spectrometer. As both the exit aperture for the interferometer and the entrance aperture for the flat field polychromator, its size, shape, and position affect the resolution and throughput of both the interferometric and dispersive sections of the MMS.

\section{Dynamic Design Considerations}

This section discusses MMS design aspects that depend upon dynamic factors such as interferometer mirror movement, detector array scanning, and mirror/PDA coordination. In the dispersive mode, it is essential that the AC modulations produced by the interferometer are nullified and that only the DC light level is detected. In the interferometric and multichannel/multiplex modes, the opposite is true; the AC modulations produced by the interferometer must be preserved, and the DC light level must be rejected.

\section{Dispersive Mode}

In this mode, the spatial and temporal integration capabilities of the PDA are used to achieve the $\mathrm{DC}$ detection/AC rejection required. To achieve spatial integration the moving mirror, M1, is positioned close to the beamsplitter. At this extreme mirror position, the rings of the circular interference pattern contract to very small diameters. ${ }^{59}$ By averaging all light intensity across the pixel surface, each pixel removes all spatial variations except for the small central fringe. 
To achieve temporal integration, the moving mirror, $\mathrm{M} 1$, is scanned rapidly relative to the PDA readout rate. In the dispersive mode the PDA has an array readout rate and a temporal sampling rate of $30 \mathrm{~Hz}$. The resulting pixel readout rate is $15 \mathrm{kHz}$ (see Table 2). In contrast to the $30 \mathrm{~Hz}$ sampling rate, typical light-modulation rates are $1,000 \mathrm{~Hz}$ or greater. Therefore, at least 35 modulations are accumulated during a single pixel integration period. Consequently, the effect of a single modulation as a fraction of the total integrated signal is reduced. Calculations have shown that the residual modulation of the central fringe is less than $2 \%$ of the integrated intensity in the central fringe. The modulations are further reduced by the spatial integration discussed above. It should be pointed out that this scheme is only one of several that could cancel the interferometer modulations in the dispersive mode. Given the dynamic feedback system for the interferometer moving mirror, this scheme provides the best optical throughput for the dispersive mode.

\section{Interferometric and Multichannel/Multiplex Modes}

In these modes, rapid PDA readout is required to achieve AC detection. In both modes, the helium-neon laser reference signal initiates entire scans of the PDA instead of initiating individual sample pulses as in a conventional interferometer. In addition, the basic $\mathrm{HeNe}$ beat frequency is not used, but it is instead multiplied by a factor of 4 , from $800 \mathrm{~Hz}$ to $3.2 \mathrm{kHz}$. Consequently, the PDA has an array readout rate and a temporal sampling rate of $3.2 \mathrm{kHz}$ in these two modes. By comparison, the interferometer modulates source light at frequencies from 630 to $1,260 \mathrm{~Hz}$ for wavelengths from $800 \mathrm{~nm}$ to $500 \mathrm{~nm}$ respectively. The required readout rate for individual pixels in these modes is $2,000,000$ pixels per second.

The rapid array readout rate $(3.2 \mathrm{kHz})$ fulfills two criteria for $\mathrm{AC}$ detection. First, the Nyquist criteria is satisfied if unaliased spectral acquisition is required. Increasing the sampling rate from $800 \mathrm{~Hz}$ to $3.2 \mathrm{kHz}$ expands the sample-limited free spectral range from $0-7,901 \mathrm{~cm}^{-1}(\infty$ $1,266 \mathrm{~nm})$ to $0-31,606 \mathrm{~cm}^{-1}(\infty-316.4 \mathrm{~nm})$. Second, and more importantly, the rectangular time-integration window of the PDA is kept sufficiently short relative to the periods of the 
interferometer-induced modulations. ${ }^{60,61}$ The PDA integration window is $0.3125 \mathrm{msec}$, versus light modulation periods of $1.012 \mathrm{msec}$ for $500 \mathrm{~nm}\left(20,000 \mathrm{~cm}^{-1}\right)$ and $1.580 \mathrm{msec}$ for $800 \mathrm{~nm}(12,500$ $\mathrm{cm}^{-1}$ ). The interferometer-induced modulations are thus successfully acquired without undue attenuation. This second point is important when data is acquired at any alias.

The rapid pixel readout rate in the interferometric and multichannel/multiplex modes places some severe requirements on the analog components of the data acquisition system. Because the PDA is read out serially, the analog components must be capable of handling the $2 \mathrm{MHz}$ pixel output rate, and also the rapid intensity transitions between pixels. For a given optical channel (pixel) however, A/D conversion rates occur at a maximum of $3.2 \mathrm{kHz}$. The DC portion of the optical channel interferogram is then removed by analog and digital filters. Spectrometric options and specifications for the interferometric and multichannel/multiplex modes are listed in Table 3.

Changing the MMS between modes is totally under computer control and is very rapid, except for changing the transfer aperture. Changing A1 is done manually in the present instrument, taking only a few seconds. The rest of the reconfiguration process takes less than a second.

\section{The MMS Instrument Control and Data Acquisition}

\section{System}

A schematic of the MMS instrument control and data acquisition system is shown in Figure 6. This system provides the control and synchronization of the interierometer, the PDA, and the A/D subsystems for all modes of operation. The Interferometer Controller Board is a 7199 Nicolet controller board, lab-modified for use in the MMS. This board provides the interferometer with direct closed-loop control of the mirror movement. It also provides white-light and laser references signals to the Spectrometer Controller Board. The Parallel Board transfers gross mirror 
Table 3. Interferometric and Multichannel/Multiplex Modes: Options and Specifications

Interferograms:

Spectral bandpass, per pixel:

Aliasing:

FFT:

Apodization:

Phase correction:
Symmetric, 128 to 8,192 points

1.2 to $6 \mathrm{~nm}$, corresponding to the transfer aperture widths.

1 to 256 , in powers of 2 .

8 to 8,192 complex points, 16-bit integer

None, Gaussian, or Hamming (cosine).

None 
control information, such as scan limits and scan velocity, from the DEC LSI-11 computer to the Interferometer Controller Board.

The Array Drive Board is an RC-1024S evaluation board from Reticon Corp. It has been lab-modified to permit external control and clocking of the PDA.

The Array Interface Board provides interfacing between the digital and analog electronics of the Array Drive Board and the rest of the MMS. For digital signals, the Array Interface Board provides optical isolation for the control signals from the Spectrometer Controller Board to the Array Drive Board. For the PDA analog output signal, the Array Interface Board provides the signal amplification and the fast sample-and-hold function required between the Array Drive Board and the A/D Board. To accommodate the $2 \mathrm{MHz}$ pixel output rate of the PDA in the interferometric and multichannel/multiplex modes, the laboratory-built gain amplifiers and sample-and-hold amplifier have $50 \mathrm{nsec}$ rise times, and the sample-and-hold amplifier has an acquisition time of $<100 \mathrm{nsec}$.

The Spectrometer Controller Board is the electronic heart of the MMS. It initiates, controls, and synchronizes the interferometer mirror scans, the PDA readouts, and the A/D conversions. Prior to data collection, the Spectrometer Controller Board receives spectrometric parameters from the CPU and stores them on board. The parameters include operating mode, array integration time, system clock frequency, mirror scan delay, mirror scan length, and data acquisition interval. After the CPU initiates data collection, the Spectrometer Controller Board is in total control of the MMS. The Spectrometer Controller Board is laboratory designed and fabricated, and is built on a DEC DRV11-P prototyping module.

The A/D converter, model 1030, from ADAC Corp. has 12-bit resolution, and a conversion time of $20 \mu \mathrm{sec}$. It has a maximum conversion rate of $50 \mathrm{kHz}$, but is limited to less than $25 \mathrm{kHz}$ by the bandwidth of the LSI-11 bus. The oscilloscope is used to provide a real-time monitor of the PDA signal going into the A/D converter, and for spectrometer tuning purposes.

The CPU, a DEC LSI-11, controls the MMS by means of the Parallel Board and the Spectrometer Controller Board. Software for instrument control and data acquisition is down-line loaded from a host LSI-11 computer. Experimental data, the spectrometric parameters and 


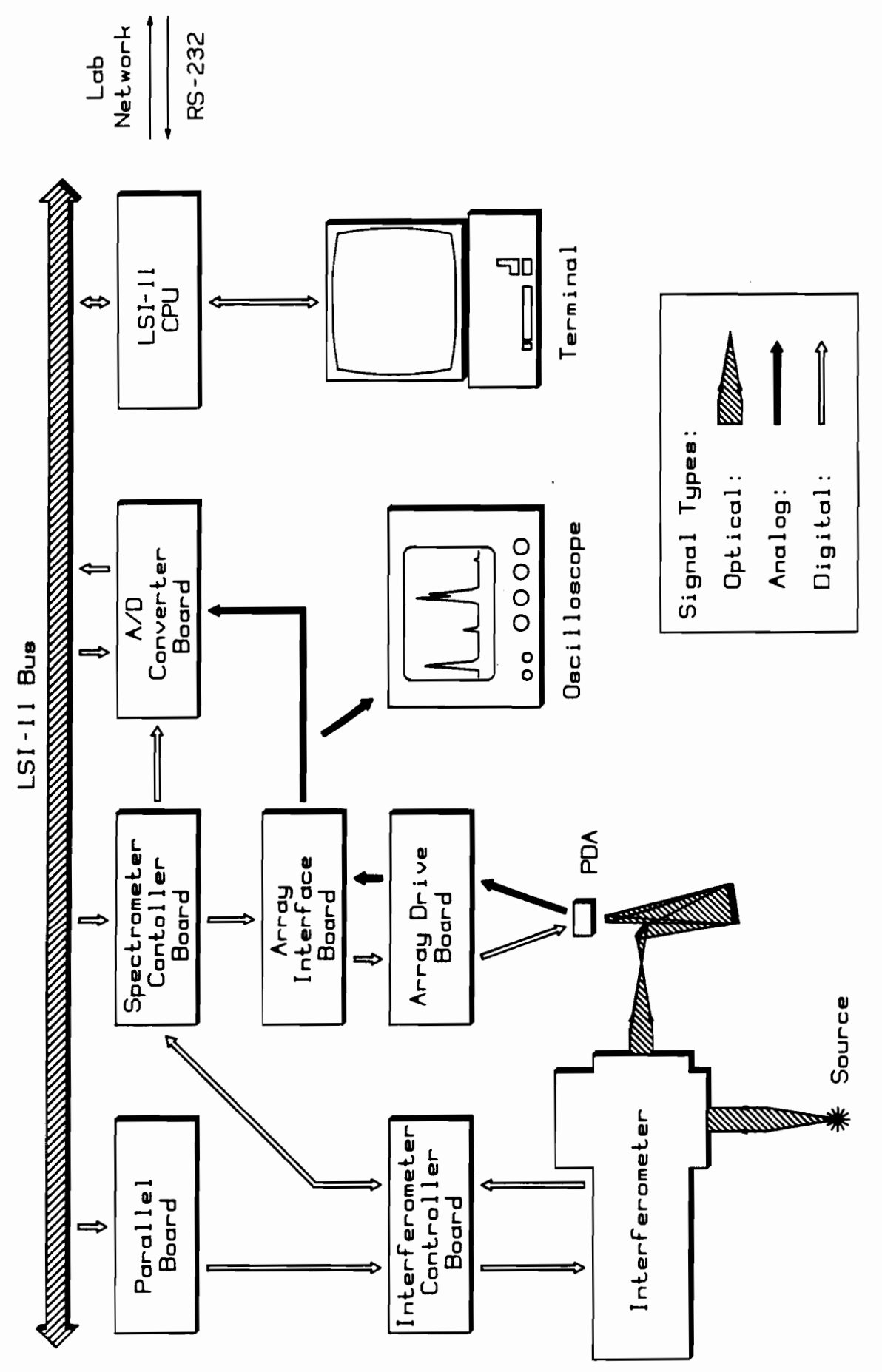

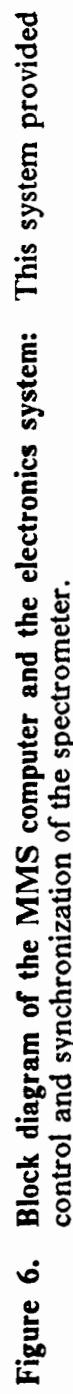


acquired data, are up-line transferred to the host computer for storage, transforming, and plotting. Table 4 lists the specifications for the computer systems. 


\section{Table 4. Computer Systems}

Spectrometer Control and Data Acquisition Computer:

CPU:

Manufacturer:

Digital Equipment Corp., Maynard, MA

Model:

Memory:

A/D converter:

Manufacturer:

Model:

Resolution

DEC LSI-11/03, 16-bit, $2.5 \mathrm{MHz}$

Conversion time:

Conversion rate $(\max )$ :

58 kbytes, static RAM

Parallel board:

ADAC Corp., Woburn, MA

1030

12-bit

$20 \mu \mathrm{sec}$

$50 \mathrm{kHz}$

DEC DRV11 parallel line unit.

Data Analysis and Data Storage Computer System:

Processor:

Memory:

Disks:

Graphics terminal:

Plotter:

Software and specifications:

System software:

Instrument control, data acquisition, data analysis, and graphics software:
DEC LSI-11/2, 16-bit

58 kBytes, DRAM

AED 6200 Floppy disk system quad drive

2.4 Mbytes total disk storage

DEC VT-100 with

Retrographics Tek 4010 emulator

Hewlett-Packard 7475A

PolyForth, Forth Inc.

Real-time, multiuser, multitasking operating system and language.

Laboratory developed in PolyForth. 


\section{Theory: Interpretation of MMS Spectra}

\section{Aliasing Factor}

Controlled aliasing is often used when acquiring data in the interferometric and multichannel/multiplex modes. When this is done, it is necessary to express the degree of aliasing and the spectral bandwidth for which the data is acquired. ${ }^{22,33}$ To do this, an aliasing factor, [3:4] for example, accompanies each interferometric spectrum. The "4" specifies the degree of aliasing, indicating that only every fourth data point was acquired in the interferogram. It also indicates the spectral range is reduced by a factor of 4 , from the MMS maximum of $31,605.6 \mathrm{~cm}^{-1}$ to $7,901.4$ $\mathrm{cm}^{-1}$. With an alias of 4 and no optical filter, the following sample-limited spectral ranges would be superimposed upon one another:

\begin{tabular}{rrrll} 
& \multicolumn{2}{c}{ Spectral Range } & Direction & Alias Factor \\
1) & 0.0 to & $7,901.4 \mathrm{~cm}^{-1}$ & (forward), & {$[1: 4]$} \\
2 ) & $15,802.8$ to & $7,901.4 \mathrm{~cm}^{-1}$ & (reverse), & {$[2: 4]$} \\
3 ) & $15,802.8$ to & $23,704.2 \mathrm{~cm}^{-1}$ & (forward), & {$[3: 4]$} \\
4 ) & $31,605.6$ to $23,704.2 \mathrm{~cm}^{-1}$ & (reverse), & {$[4: 4]$}
\end{tabular}


The spectral ranges are listed with their respective alias factors. "Forward" indicates that the wavenumbers numbers in the transformed spectrum increase from left to right. "Reverse" indicates an increase from right to left.

The " 3 " in the alias factor indicates the specific sample-limited spectral range of the data. As an example, most of the interferometric mode data in this chapter were acquired using pixel 159 as the detector. Pixel 159 monitors a spectral band at $590 \mathrm{~nm}$, or $16,950 \mathrm{~cm}^{-1}$, which is within spectral range " 3 ", from $15,802.8$ to $23,704.2 \mathrm{~cm}^{-1}$.

\section{Resolving Power, Resolution, and Spectral Bandwidth}

Resolution is difficult to compare between dispersive and interferometric instruments. It is fairly constant in wavelength for dispersive instruments, whereas it is fairly constant in wavenumber for interferometric instruments. Resolving power, however, can be computed for both types of spectrometers, and is the most useful parameter for comparing different types of spectrometers. Resolving power cannot be considered a constant for wide spectral ranges, and must usually be calculated at each line position.

The resolving power, $R$, of a spectrometer is given as

$$
R=\frac{\lambda}{\delta \lambda}=\frac{\sigma}{\delta \sigma}
$$

where $\lambda$ and $\delta \lambda$ are spectral position and resolution in wavelength (nm), and $\sigma$ and $\delta \sigma$ are spectral position and resolution in wavenumber $\left(\mathrm{cm}^{-1}\right)$. Also

$$
\lambda=\frac{1}{\sigma}
$$

Two relationships useful for converting resolutions or spectral bandwidths are as follows:

$$
\delta \lambda=\frac{\delta \sigma}{\sigma^{2}}
$$




$$
\delta \sigma=\frac{\delta \lambda}{\lambda^{2}}
$$




\section{Experimental}

\section{MIP}

The MIP served as a convenient atomic emission source. Like the inductively coupled plasma (ICP) and the direct-current plasma (DCP), the MIP is composed of a plasma torch, plasma power supply, nebulizer, sample pump, and gas flow system. The MIP is therefore potentially subject to many of the same source noises found in the ICP and DCP.

Figure 7 shows a sectional view of the MIP cavity, torch, and nebulizer used in these experiments. The MIP setup is a simplified version of the one described by Long and Perkins. ${ }^{62}$ The cavity is a high-efficiency capacitively-coupled $\mathrm{TM}_{010}$ design, after Matus, Boss, and Riddle.63 It is a Beenakker cavity, ${ }^{64}$ utilizing a translatable disk electrode and quartz-rod tuning. The microwave generator was connected directly to the cavity, with no microwave circulator or external load. The following components were chosen because of their low noise characteristics. The torch is a tangential flow design, after Lysakowski. ${ }^{65}$ This torch produces a toroidal, self-centering plasma of high stability. The nebulizer is a fritted disk type, after Layman and Lichte. ${ }^{66}$ It minimizes nebulizer noise ${ }^{49}$ by producing a small mean droplet size with a narrow droplet size distribution. Table 5 lists the MIP apparatus specifications. 


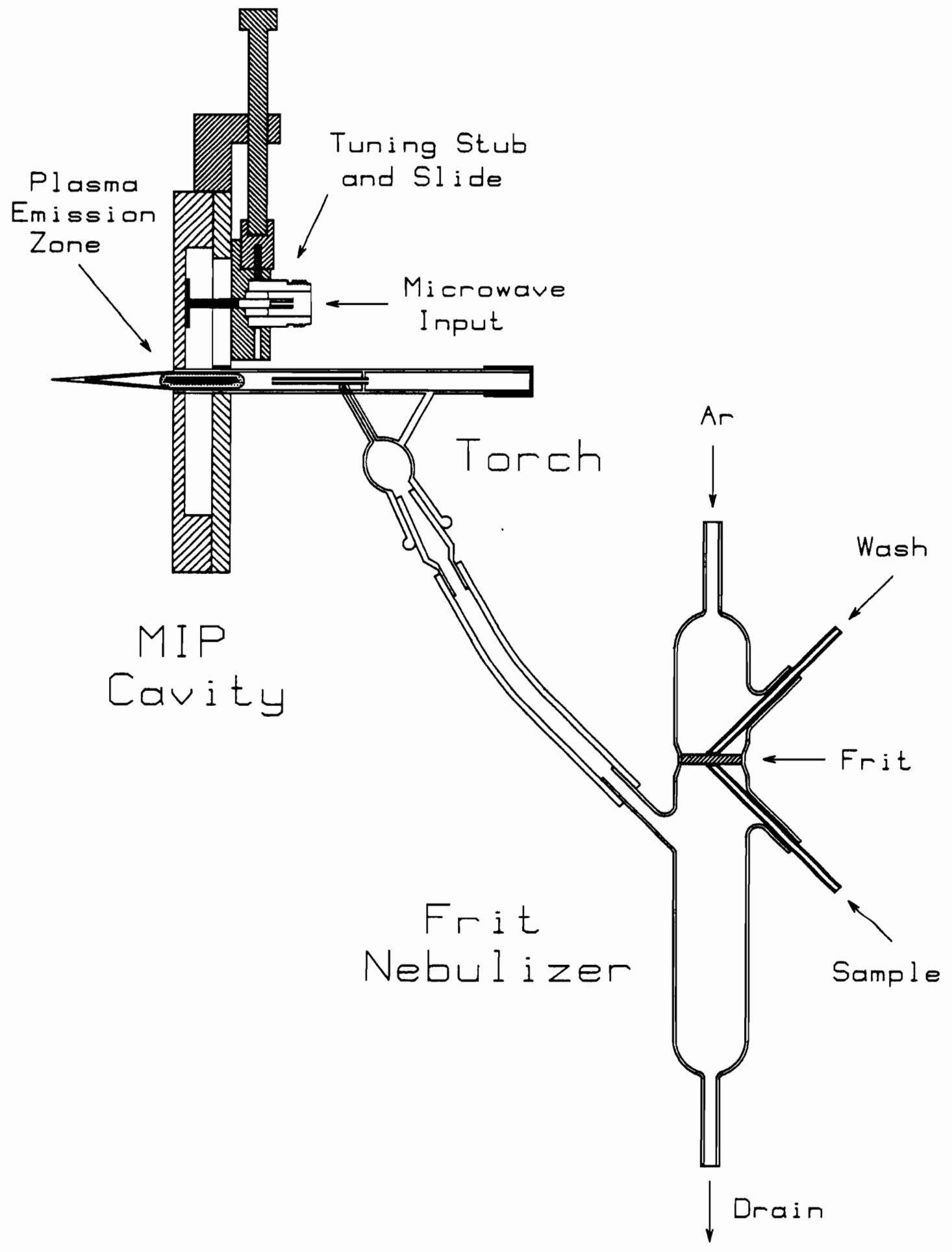

Figure 7. Cross-section of the MIP apparatus: The MIP cavity, torch, and nebulizer are shown. 
MIP Source and System:

Cavity:

Nebulize

Microwave Generator:

Manufacturer:

Model:

Power:

Frequency:

Load:

High-efficiency $\mathrm{TM}_{010}$, laboratory built

Tangential flow, laboratory built

Fritted disk, laboratory built

Kiva Instrument Corp., Rockville, MD

MPG-4M

$120 \mathrm{~W}$

$2.450 \mathrm{GHz}$

$50 \mathrm{ohm}$

Tungsten White-Light Spectral Irradiance Source: Spectral Irradiance Head

Manufacturer:

Model:

Description:
Lamp Monitor and Control Manufacturer:
Model:

EG\&G Gamma Scientific, San Diego, CA

RS-10A

Tungsten broad band spectral irradiance source. Correlated color temp: $2856, \pm 20 \mathrm{~K}$. Spectral range: 300 to $>1100 \mathrm{~nm}$.

EG\&G Gamma Scientific

RS-1 
The MIP setup permitted direct introduction of aqueous samples. The plasma was sustained by the Ar flow through the nebulizer. The plasma extended approximately $1 \mathrm{~cm}$ beyond the cavity. Observation was side-on, with the plasma horizontal. In this configuration, the base of the plasma and the face of the cavity were parallel to the long dimension of the transfer aperture, A1. The observation height was 0.5 to $1 \mathrm{~mm}$ beyond the cavity face. See Table 6 for the MIP operating conditions.

\section{Tungsten Source}

A white, broadband spectral source was needed to characterize the spectral filtering capability provided by the PDA and grating in the interferometric and multichannel/multiplex modes of operation. The tungsten spectral irradiance source listed in Table 5 was used.

\section{Solutions}

The $\mathrm{NaCl}$ and the $\mathrm{KCl}$ salts used were reagent grade. Stock $1000 \mu \mathrm{g} / \mathrm{mL}$ solutions were prepared using distilled/deionized water. Other solutions were prepared by dilution using distilled/deionized water. 
Table 6. MIP Operating Conditions

Plasma Parameters:

Plasma support gas:

Gas flow:

Nebulizer pressure:

Solution delivery rate:

Forward power:

Reflected power:

Ar

$2 \mathrm{~L} / \mathrm{min}$

$30 \mathrm{psi}$

$0.100 \mathrm{~mL} / \mathrm{min}$

$70 \mathrm{~W}$

$\sim 0 \mathrm{~W}$

Plasma observation geometry:

Plasma orientation:

Observation angle:

Horizontal

Observation height:

Side-on (radial)

0.5 to $1.0 \mathrm{~mm}$ 


\section{Results}

Several atomic emission spectra for the dispersive, interferometric, and multichannel/multiplex modes are shown, demonstrating the feasibility of the MMS to obtain AES data. In addition, several interferometric spectra of the white-light tungsten source are included to characterize the spectral filtering and the filter response function of the MMS. Dispersive AES spectra were taken of a sample with $500 \mu \mathrm{g} / \mathrm{mL}$ of $\mathrm{Na}$ and $500 \mu \mathrm{g} / \mathrm{mL}$ of $\mathrm{K}$. Due to clogging problems with the nebulizer frit, a more dilute sample $(100 \mu \mathrm{g} / \mathrm{mL})$ was used for the interferometric and multichannel/multiplex mode AES spectra.

\section{Dispersive Mode AES Spectra}

Dispersive mode spectra of the MIP are shown in Figures 8,9 , and 10. Figure 8 is a blank spectrum, showing plasma background due to Ar and water-derived emissions. Possible line assignments for this spectrum are listed in Table 7. Figure 9 shows the spectrum for the $\mathrm{Na}$ and $\mathrm{K}$ sample. Figure 10 shows the difference spectrum. The MMS operating parameters for these spectra are listed in Table 8. 


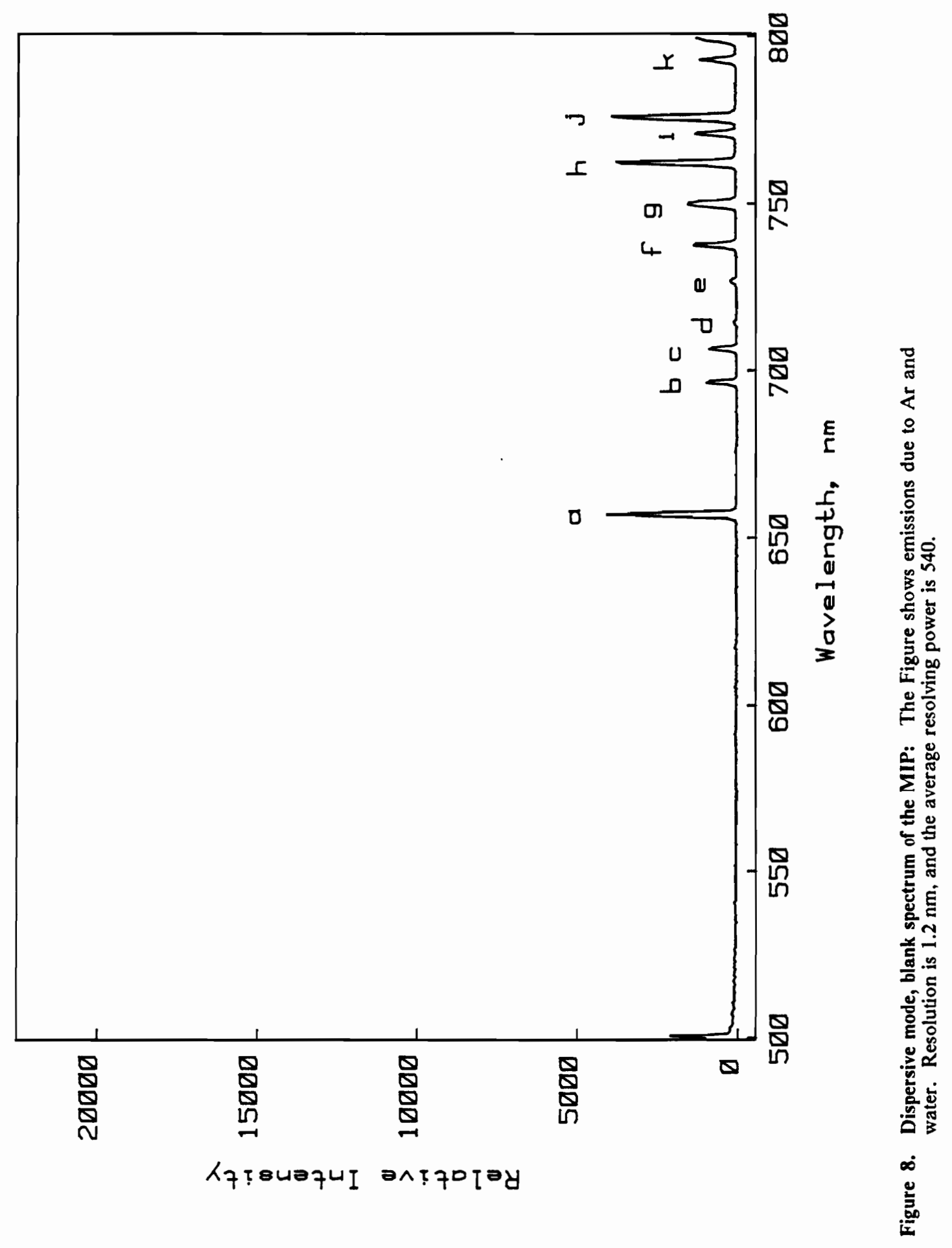

Results 


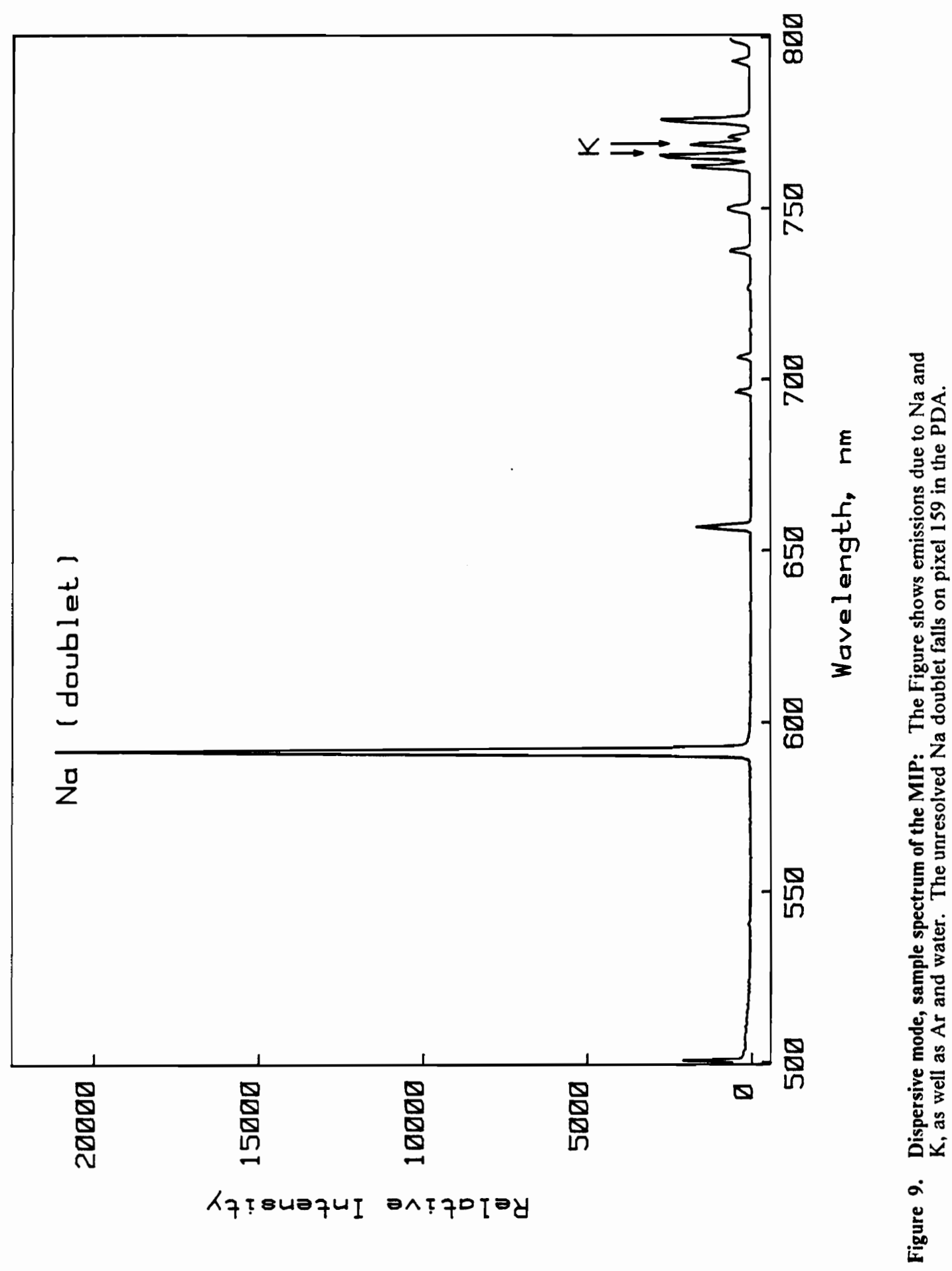

Results 


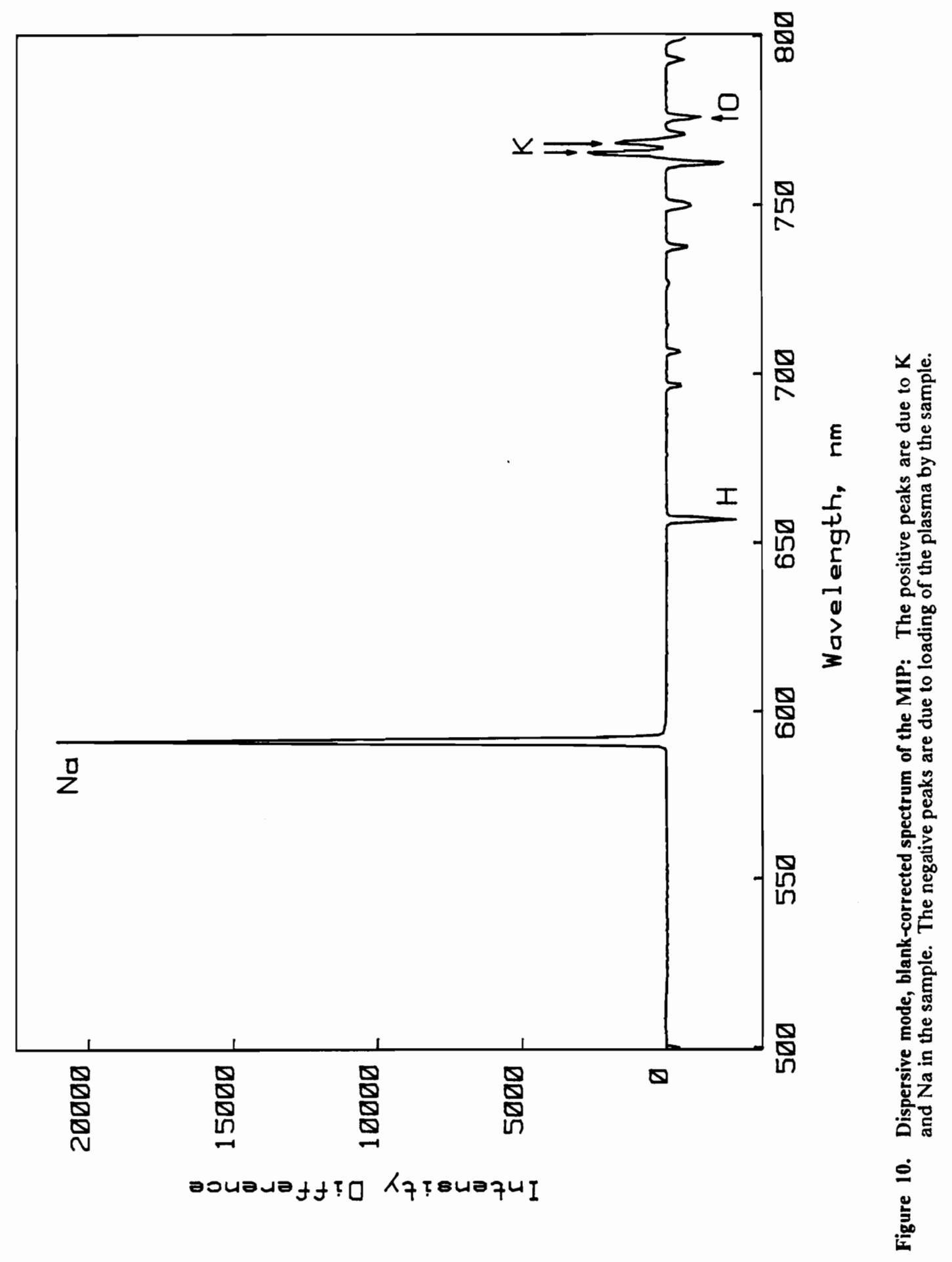


Table 7. Reference Data for MIP Line Assignments

A. Candidate lines for the plasma background, $\mathrm{Ar}, \mathrm{H}$, and $\mathrm{O}: a, b$

Line

a)

b)

c)

d)

e)

f)

g)

h)

i)

j)

k)

Species

Wavelength

$(\mathrm{nm})$

Intensity

656.27

656.28

1000

2000

696.54

400

703.22

706.72

100

400

714.90

30

727.29

100

735.33

737.21

738.40

100

100

400

750.39

700

200

762.89

50

763.51

500

772.37

200

200

1000

300

100

777.41

777.54

100

789.11

400

B. Reference line values for $\mathrm{Na}$ and $\mathrm{K}:{ }^{a, c}$

Line

1) and 2)

1)
Species

$\mathrm{NaI}$

K I

K I

794.82

Wavelength

589.00

589.59

766.49

769.90
Intensity

9000

5000

9000

5000

- Literature values from references 67 and 68.

- The $\mathrm{H}, \mathrm{O}$, and Ar lines are from a gas discharge source.

c $\mathrm{K}$ and $\mathrm{Na}$ lines are from an arc source. 
Table 8. Common MMS Operating Parameters for Figures 8, 9, and 10

Experimental parameters:

Source:

Mode:

Spectral Range:

MIP

Spectral bandwidth:

Dispersive

$500-800 \mathrm{~nm}$.

Points per Array Scan:

$300 \mathrm{~nm}$

512

Transfer aperture:

Resolution:

$1.000 \times 0.050 \mathrm{~mm}$.

Average resolving power:

$1.2 \mathrm{~nm}$

540

Integration time:

$33.3 \mathrm{msec}$

Summed scans per spectrum:

Exposure time, total:

$333 \mathrm{msec}$. 
These spectra demonstrate the feasibility of the dispersive mode. Analyte and background data are obtained rapidly and simultaneously and over a wide spectral range. As shown by Figure 10, difference spectra can be particularly illuminating. The positive and negative peaks may indicate respective increases or decreases in sample components. In this case, the positive peaks do indicate an increase in the $\mathrm{Na}$ and $\mathrm{K}$, but the negative peaks indicate a loading of the plasma caused by the fairly concentrated sample.

\section{Interferometric Mode White-Light Data}

Because the spectral filtering by the polychromator/PDA section is central to the concept of the MMS, characterization of the MMS filter functions was very important. Two aspects were important: the shape of an individual filter function, and the relationship between adjacent filter functions. The characterization was done using the white-light tungsten source.

Typical bandlimited interferometric data of the tungsten source are shown in Figures 11 to 14. Resolution is $7.716 \mathrm{~cm}^{-1}$ and the alias factor is [1:1]. The other MMS operating parameters for this data are shown in Table 9. Figure 11 shows the interferogram of the tungsten source at $590 \mathrm{~nm}$, acquired by monitoring diode 159 . The form of the interferogram is generally as expected, a sinc function envelope with a carrier frequency of $16,950 \mathrm{~cm}^{-1}(590 \mathrm{~nm})$. The asymmetry of the interferogram is due an optical error in the beam splitter. The error could not be removed by adjusting the mirror alignment.

Fourier transforming this interferogram produces the amplitude spectrum shown in Figure 12. This is a plot of the full sample-limited free spectral range, from 0 to $31,606 \mathrm{~cm}^{-1}$, showing the optical filtering of the MMS from a wide-band perspective. Figure 13 is a close-up of the same data. The width of the function, $6 \mathrm{~nm}$ at FWHM, is determined primarily by the product of the reciprocal linear dispersion of the polychromator grating, $\mathrm{G},(24 \mathrm{~nm} / \mathrm{mm})$ and the width of the transfer aperture, $\mathrm{Al},(0.250 \mathrm{~mm})$. The shape of function is determined by the convolution of the 


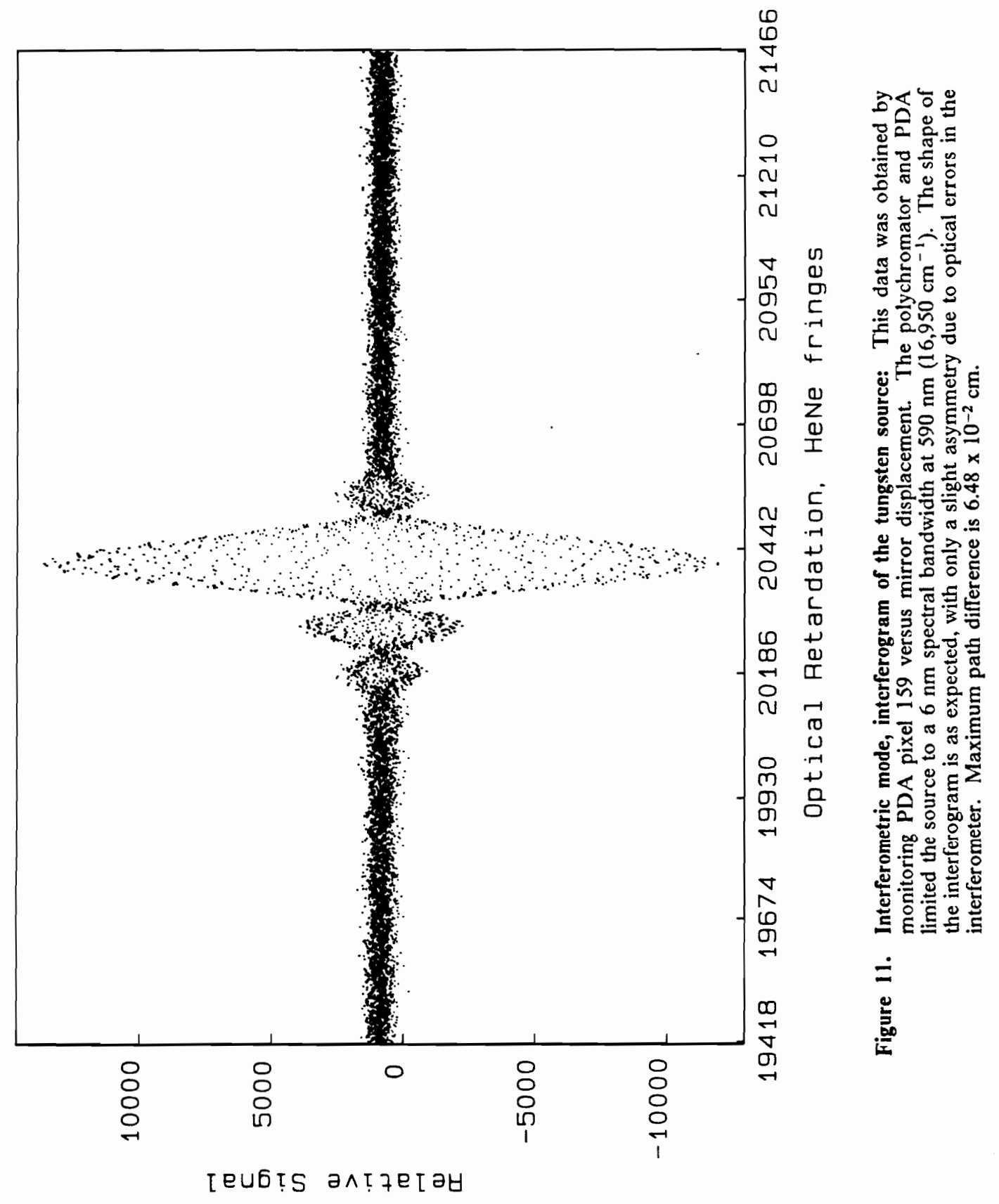




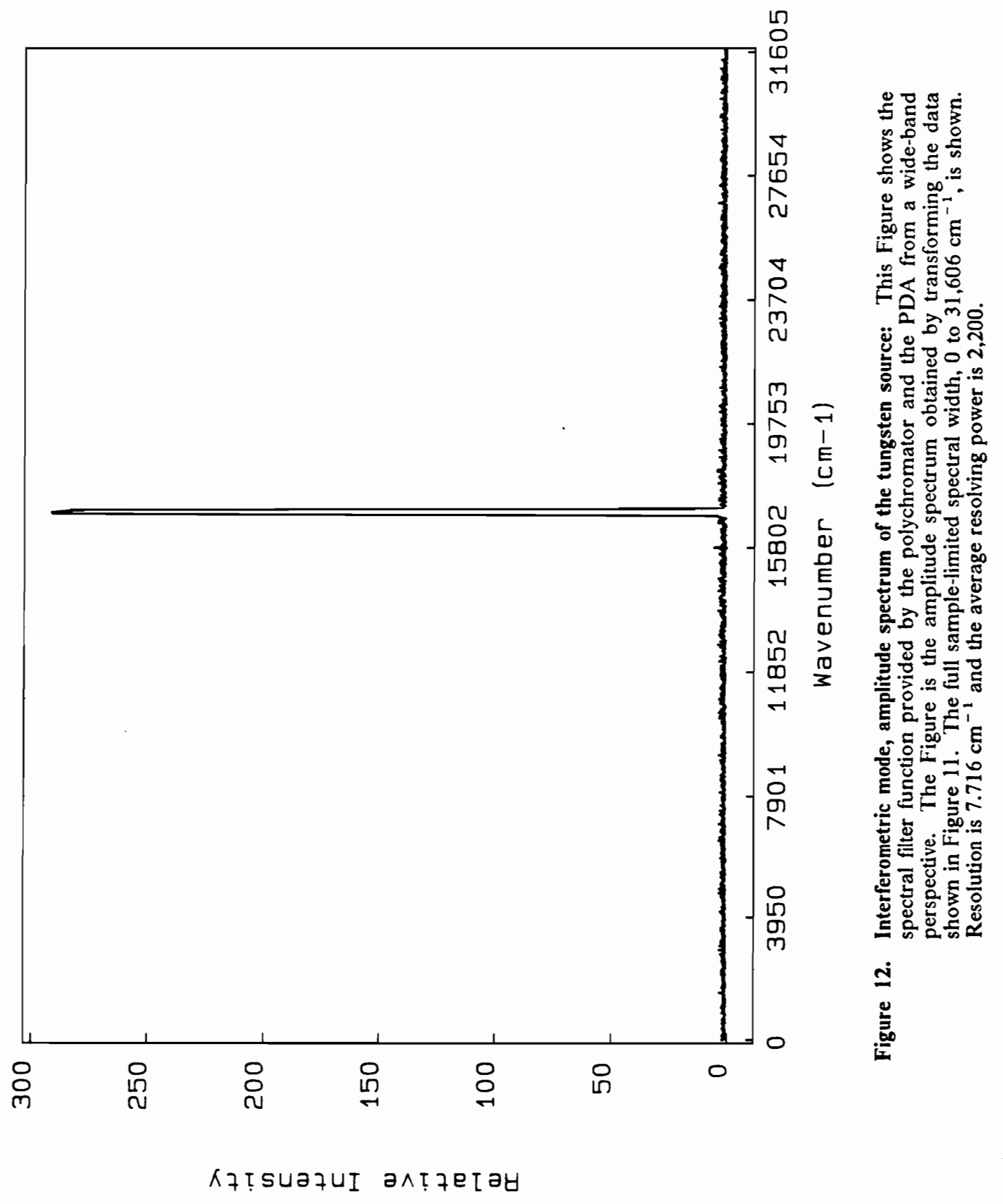

Results 


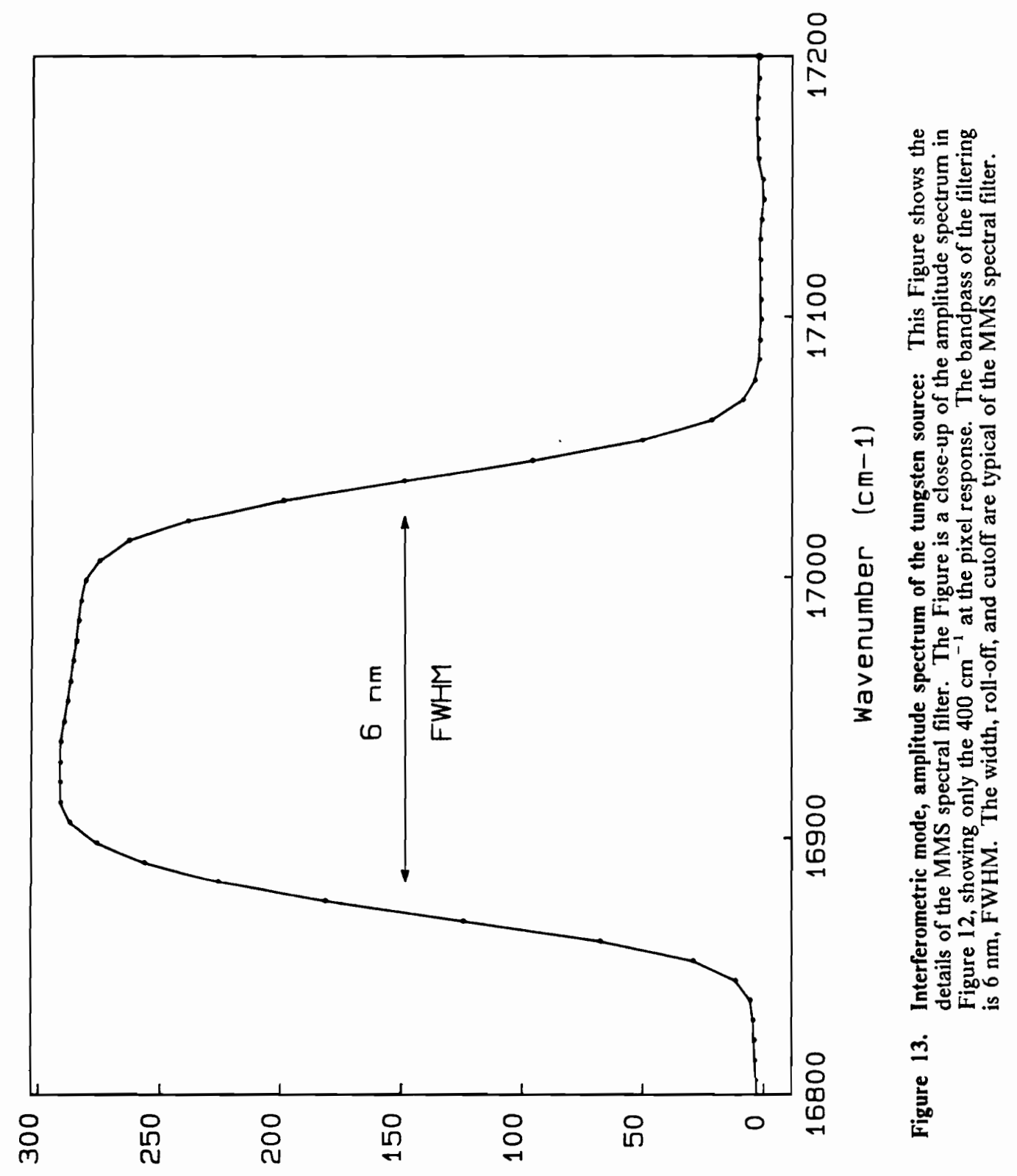

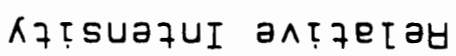




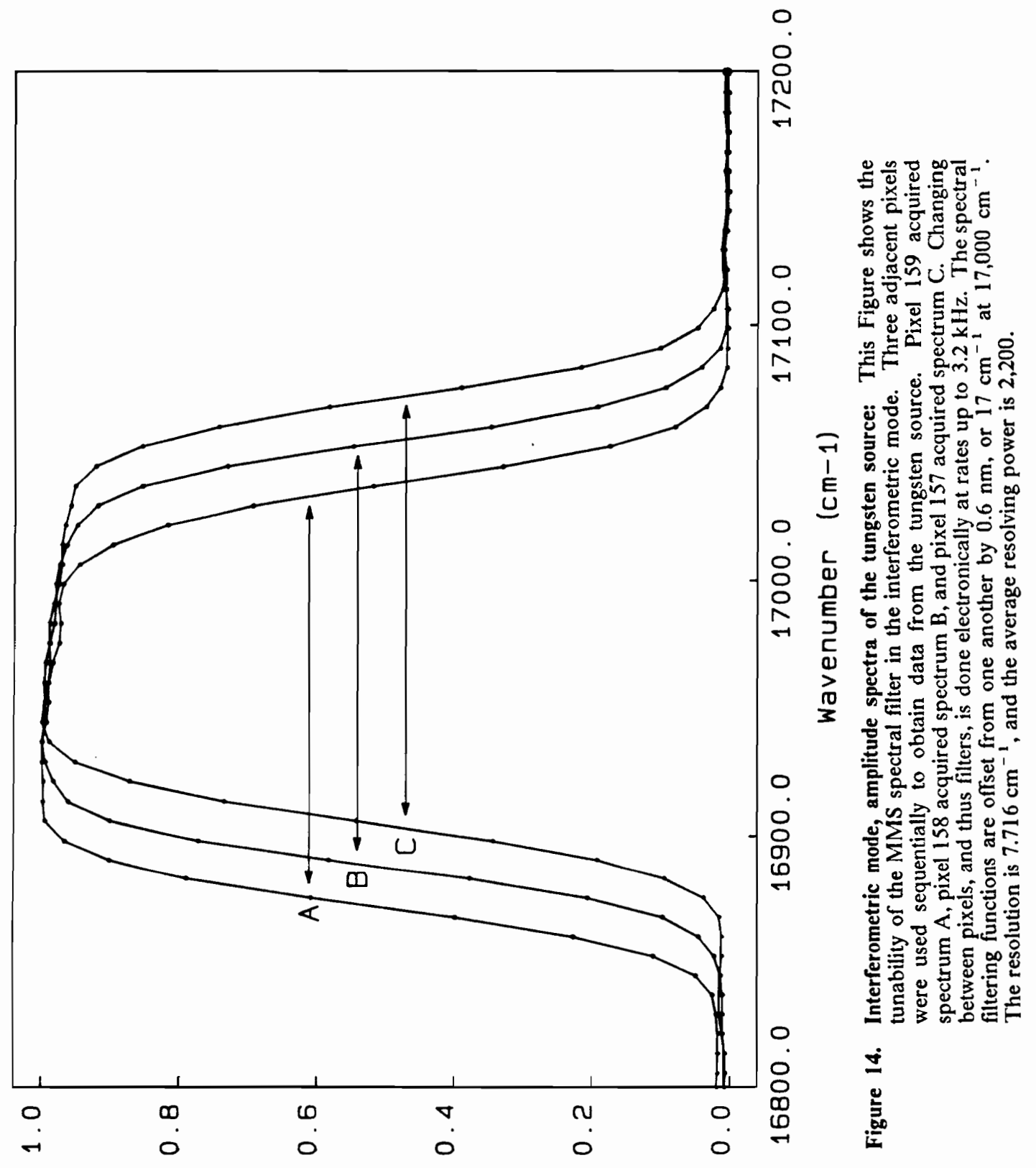

asuodsay pazt [emJon 
Table 9. Operating Parameters for Figures 11, 12, 13, and 14

Experimental parameters:

Source:

Mode:

Transfer aperture:

Spectral bandwidth:

Pixel number:

Tungsten spectral irradiance

Bandwidth center:

Alias factor:

Sampling bandwidth:

Points per

interferometer scan:

Resolution:

Interferometric

$5.000 \times 0.250 \mathrm{~mm}$.

$6.0 \mathrm{~nm}$

159

$590 \mathrm{~nm} ; 16,950 \mathrm{~cm}^{-1}$

[1:1]

$31,606 \mathrm{~cm}^{-1}$

Resolving power:

8,192 .

$7.716 \mathrm{~cm}^{-1}$.

Coadded mirror scans:

2,200

Total exposure time:

Total elapsed time:

Apodization:

100

$256 \mathrm{sec}(4 \mathrm{~min}, 16 \mathrm{sec})$

$50 \mathrm{~min}$.

Gaussian 
rectangular transmission function of the aperture Al with the trapezoidal spatial response function of a single pixel.?,17 The slight slope of the top in Figure 13 is caused by the tungsten emission curve. Modification of the shape and width of the functions is possible by changing the shape and width of the transfer aperture, Al. Spectral baseline due to scattered light should be extremely low because of the holographic grating and the immunity of FTS to stray light.

Data from 3 successive pixels was obtained to determine the relationship between adjacent filter functions. Normalized responses from pixel 159 (A), 158 (B), and 157 (C) are shown in Figure 14. The reproducibility and the spacing of the filter functions from pixel to pixel is shown. The filter-to-filter spacing is $0.6 \mathrm{~nm}$. The Figure also illustrates the flexibility in selecting the most appropriate spectral filter. Any one of 512 similar filter functions can be electronically selected by utilizing the appropriate pixel as the detector. Sub-pixel translation of the functions is possible by physically translating the PDA across the focal plane of the polychromator.

\section{Interferometric Mode AES Data}

Examples of interferometric mode AES data for the Na doublet are show in Figures 15 to 18. This data demonstrates the ability of the MMS to obtain AES data in the higher resolution interferometric mode. The data was obtained at a resolution of $1.929 \mathrm{~cm}^{-1}$ using pixel 159 as the detector; the alias factor is [3:4]. The optical filter function shown in Figures 12 and 13 was applied to the spectrum, thus suppressing all lines except the $\mathrm{Na}$ doublet. Other MMS operating parameters for these data are listed in Table 10.

Figure 15 shows the interferogram resulting from the introduction of a $100 \mu \mathrm{g} / \mathrm{mL} \mathrm{Na}$ sample into the MIP. This is the type of interferogram expected from a closely spaced doublet with fairly equal intensities in the two lines. Performing Gaussian apodization and then Fourier transforming the data produces the spectra in Figures 16, 17, and 18. 


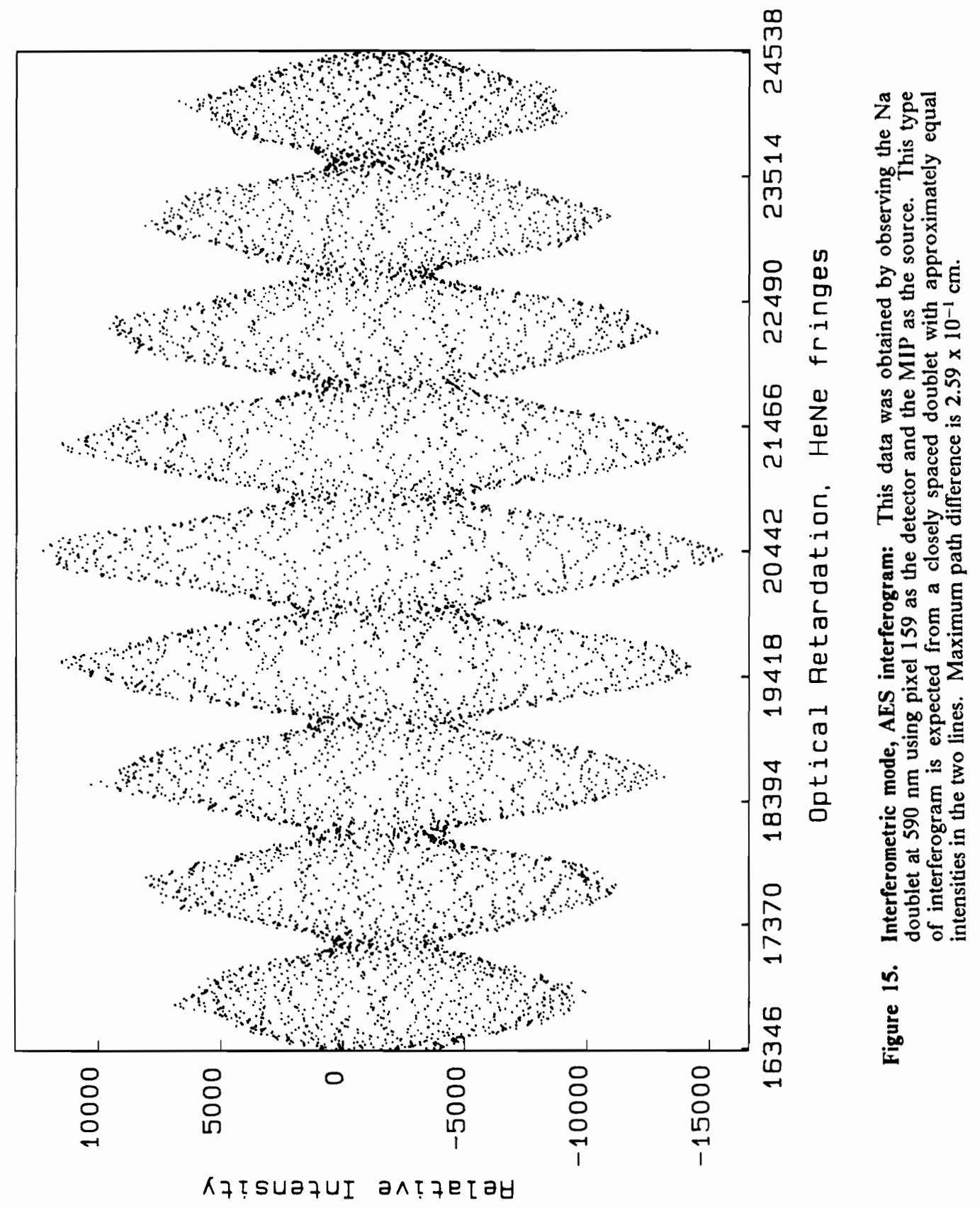




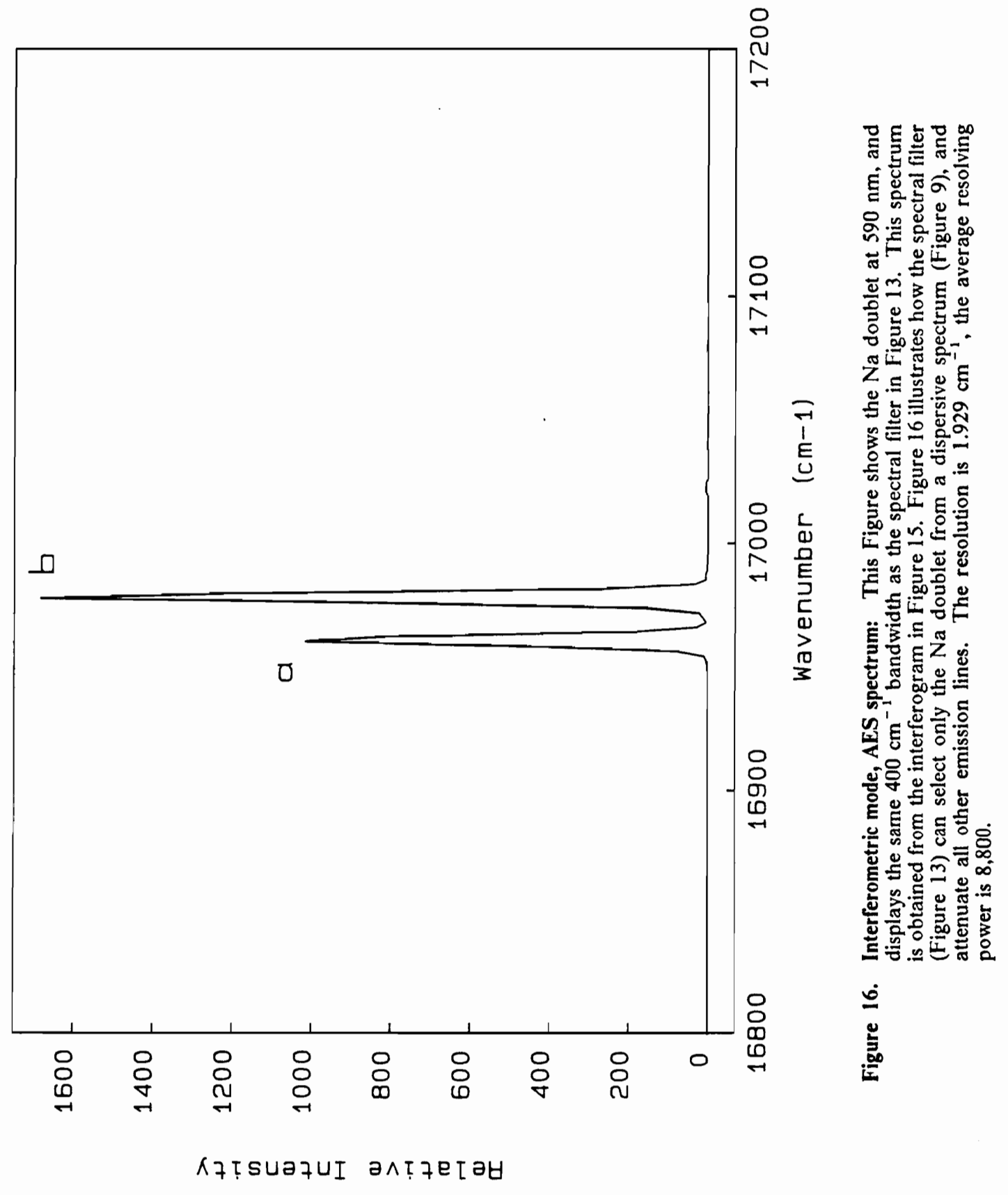

Results 


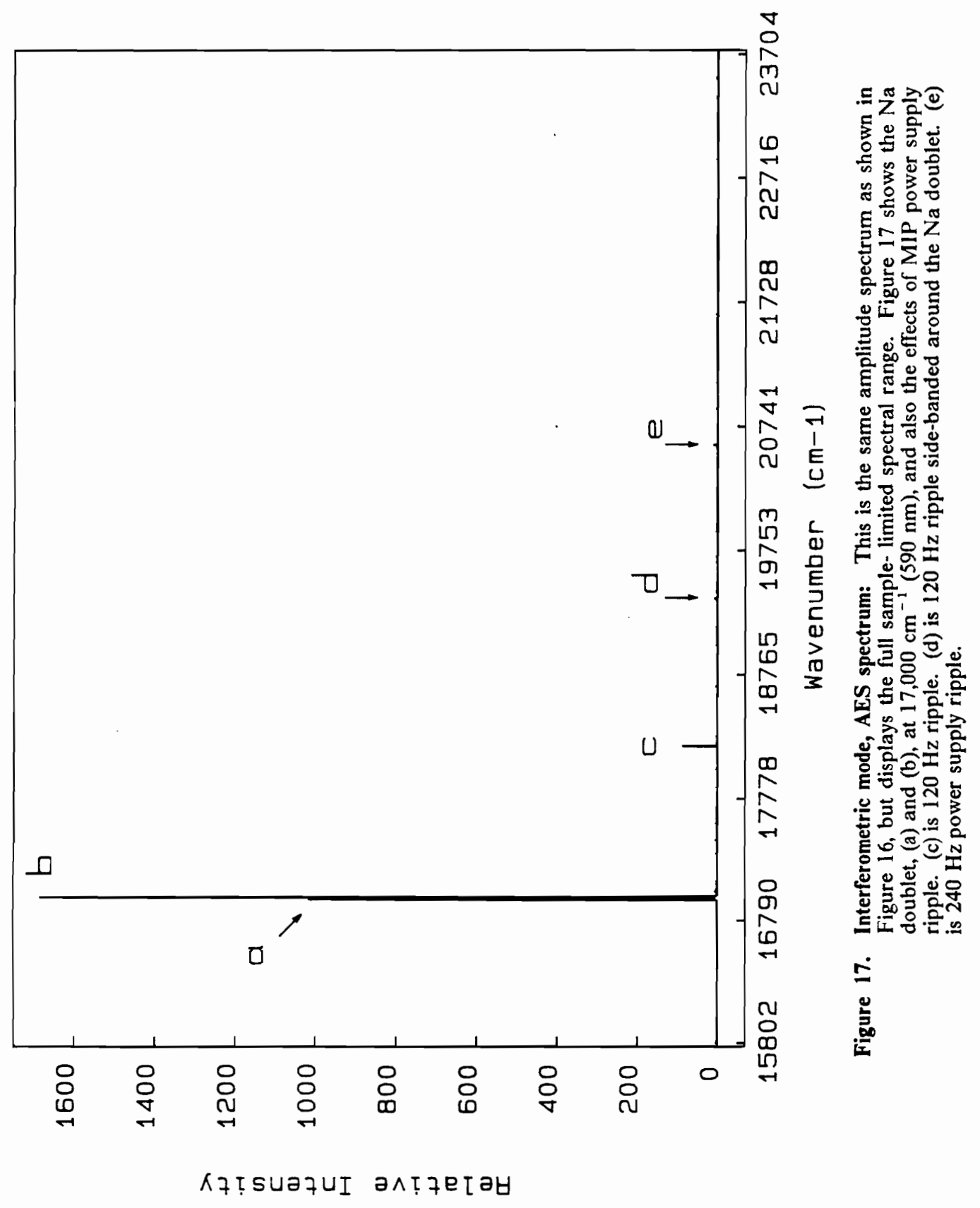




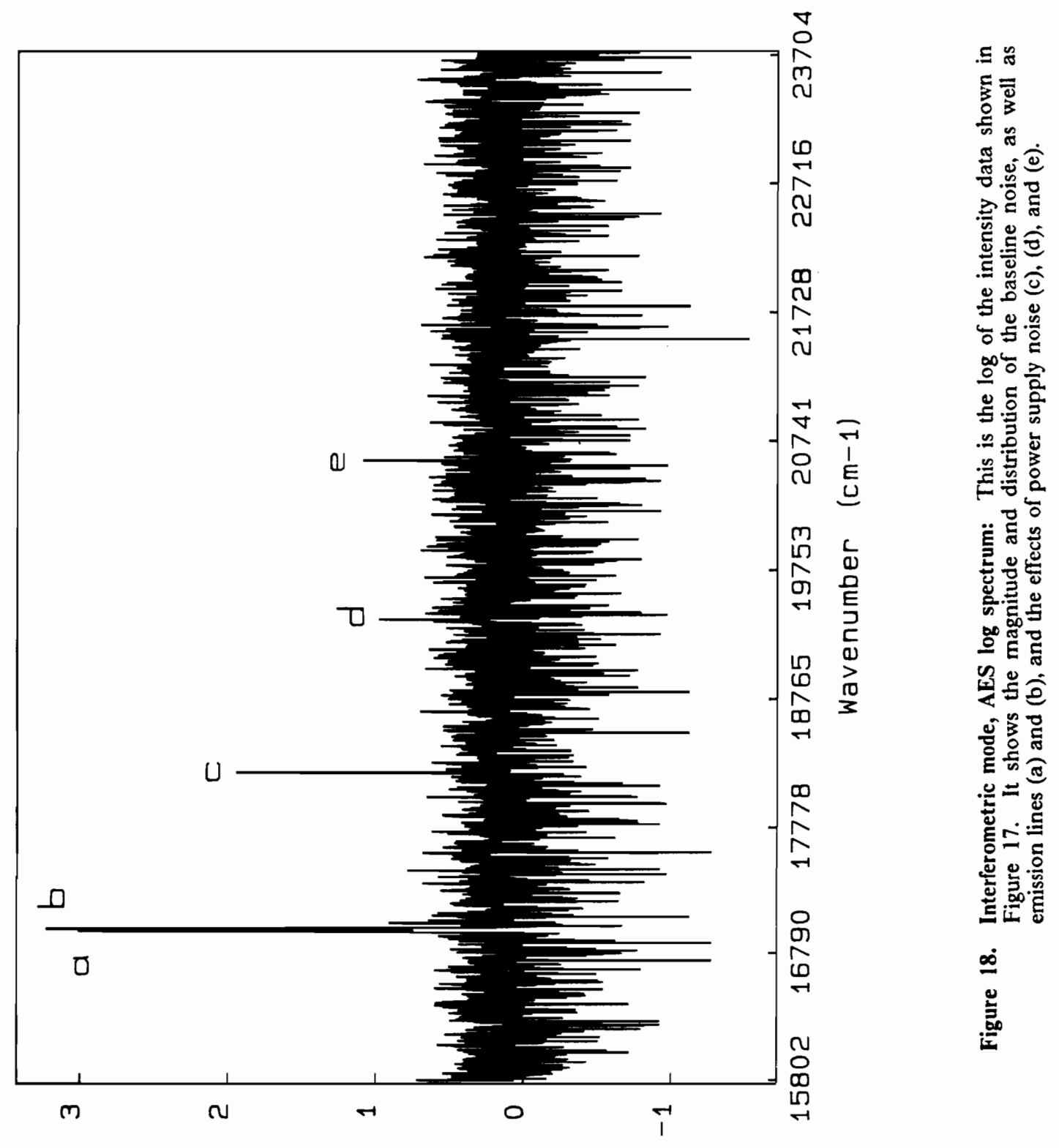

K7тรบazUI 607 
Table 10. Operating Parameters for Figures 15, 16, 17, and 18

Experimental parameters:

Source:

MIP Sample:

Mode:

Transfer aperture:

Spectral bandwidth:

Pixel number:

Bandwidth center:

Alias factor:

Sampling bandwidth:

Points per interferometer scan:

Resolution:

Resolving power:

Coadded mirror scans:

MIP

$\mathrm{Na}, 100 \mu \mathrm{g} / \mathrm{mL}$

Interferometric

$5.000 \times 0.250 \mathrm{~mm}$.

$6.0 \mathrm{~nm}$

159

$590 \mathrm{~nm} ; 16,950 \mathrm{~cm}^{-1}$

[3:4]

$7,901 \mathrm{~cm}^{-1}$

Total exposure time:

Total elapsed time:

8,192 .

$1.929 \mathrm{~cm}^{-1}$.

8,800

100

Apodization:

$256 \mathrm{sec}(4 \mathrm{~min}, 16 \mathrm{sec})$

$55 \mathrm{~min}$.

Gaussian 
Figure 16 is a close-up of the amplitude spectrum showing the same $400 \mathrm{~cm}^{-1}$ region as shown for the filter function in Figure 17. Figure 16 shows that the single Na peak at $590 \mathrm{~nm}$ in the dispersive spectrum, Figure 9, is indeed a doublet. Using Figure 16, the wavenumber and intensity of the individual lines can be determined. No detectable continuum background emission is observed; a continuum would appear as a pedestal similar to Figure 13. The noise distribution in Figure 16 appears to be white. The noise level was found by calculating the RMS noise level in the baseline away from the line..$^{50}$ The noise level was found to be 1.25 units. The $\mathrm{S} / \mathrm{N}$ for line (b) is $1,350: 1$.

Figure 17 is the full sample-limited bandwidth amplitude spectrum. This Figure demonstrates the ability of the MMS to perform noise spectrum analyses. It consequently reveals more information about the source and the spectrum. The baseline noise throughout the sampling-limited spectral range again appears to be white. There is, however, the unexpected presence of features at (c), (d), and (e). Because of the optical filtering by the PDA and polychromator, these features can be rejected as optical emissions from the sample or the plasma gas. Reinterpreting the horizontal axis of Figure 17 in Hertz, the data represents frequencies from 0 to $400 \mathrm{~Hz}$ with a resolution of $0.102 \mathrm{~Hz}$. With this perspective, features (c) and (e) are found to be $120 \mathrm{~Hz}$ and $240 \mathrm{~Hz}$ modulations. Other studies have shown that these modulations are multiplicative interferences due to ripple in the microwave power supply. Features at (d) are satellite side-bands of the $\mathrm{Na}$ lines resulting from (c) side-banding around the $\mathrm{Na}$ lines. ${ }^{48}$ In comparison, the tungsten spectrum, Figure 12, is free of these features. The lack of $60 \mathrm{~Hz}$ line noise and its various harmonics in Figure 12 demonstrates two things. First, the tungsten source is free of power supply ripple. Second, the MMS and its detection system are not subject to induced power line noise.

Figure 18 shows the $\log$ of the intensity values from Figure 17. This data format is useful when examining spectral regions displaying widely different magnitudes. The distribution of the baseline noise is confirmed to be relatively white throughout the spectrum. Comparing the baseline and line (b), the $\mathrm{S} / \mathrm{N}$ ratio of approximately $10^{3}$ is readily seen. Features (c), (d), and (e) are much more apparent and easily identified as real features, significantly above baseline. 
Several advantages of optical filtering in the MMS are graphically illustrated in Figures 17 and 18. Many plasma emission spectra display thousands of atomic lines. If such a spectrum were obtained with an interferometer without optical filtering, every atomic line in the spectrum would exhibit satellites similar to (d). This would greatly complicate interpretation of the spectrum and possibly interfere with quantitation. With the MMS however, optical and non-optical signals are easily differentiated, and spectral interpretation and analyte quantitation are potentially improved. Compared to a dispersive instrument, the source fluctuations in this mode of the MMS are distributed off of the analyte line, potentially improving $\mathrm{S} / \mathrm{N}$. Additionally, a practical instrumentation problem, the plasma power supply ripple, has been identified and can be corrected.

\section{Higher Resolution Interferometric AES Spectra}

The data in Figure 19 was obtained to demonstrate resolution flexibility and controlled aliasing. This Figure shows the $\mathrm{Na}$ doublet obtained at a resolution of $0.4823 \mathrm{~cm}^{-1}$, the highest currently attainable in the MMS, and with an alias factor of [9:16]. Figure 19 shows the same 400 $\mathrm{cm}^{-1}$ region as shown the filter function plot in Figure 13 and the lower resolution interferometric $\mathrm{Na}$ emission plot in Figure 16. The resolving power in Figure 19 is 35,200, versus 490 for the dispersive mode spectrum in Figure 9, and 8,800 for Figure 16. The MMS operating parameters for this spectrum are listed in Table 11.

The ability of the MMS to extract and compress data is demonstrated by Figure 19 in conjunction with the dispersive spectrum in Figure 9. The MMS required a 512-point dispersive spectrum followed by an 8,192-point interferometric spectrum to obtain $0.5 \mathrm{~cm}^{-1}$ resolution. A total of 8,704 data points were acquired. In contrast, a normal interferometer without optical filtering may require 131,072 points $(128 \mathrm{k})$ to obtain the resolution shown in Figure 19. The data compression resulting from the use of the MMS is approximately 15:1. 


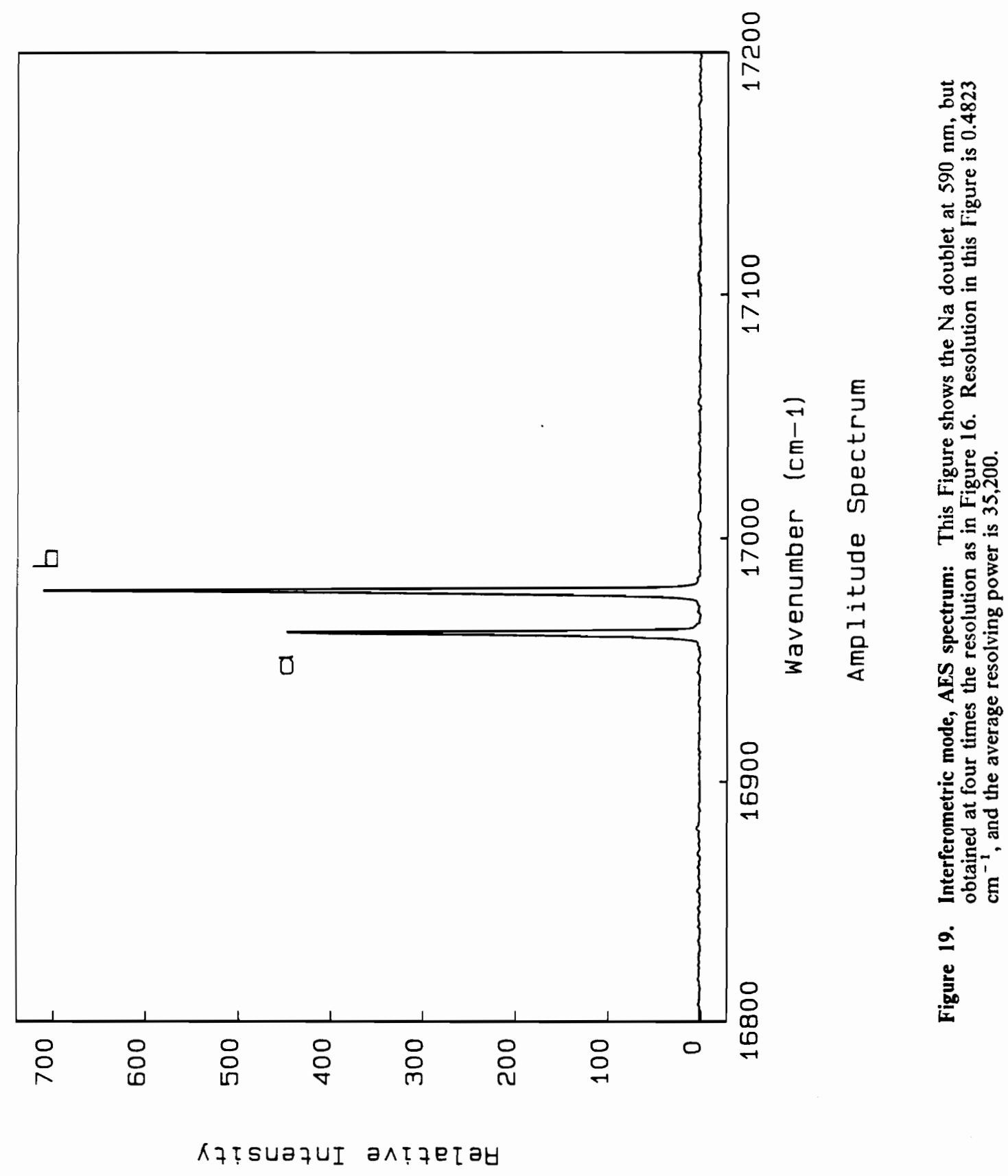

Results 
Table 11. Operating Parameters for Figure 19

\section{Experimental parameters:}

Source:

MIP Sample:

Mode:

Transfer aperture:

Spectral bandwidth:

Pixel number:

Bandwidth center:

Alias factor:

Sampling bandwidth:

Number of points:

(per interferometer scan)

Resolution:

Resolving power:

MIP

$\mathrm{Na}, 100 \mu \mathrm{g} / \mathrm{mL}$

Interferometric

$5.000 \times 0.250 \mathrm{~mm}$.

$6.0 \mathrm{~nm}$

159

$590 \mathrm{~nm} ; 16,950 \mathrm{~cm}^{-1}$

[9:16]

$1,975 \mathrm{~cm}^{-1}$

8,192 .

$0.4823 \mathrm{~cm}^{-1}$.

35,200

Coadded mirror scans:

Total exposure time:

100

Total elapsed time:

$256 \mathrm{sec}(4 \mathrm{~min}, 16 \mathrm{sec})$

Apodization:

$1 \mathrm{hr} .10 \mathrm{~min}$.

Gaussian 


\section{Multichannel/Multiplex Mode AES Data}

The feasibility of the multichannel/multiplex mode is demonstrated by Figures 20, 21, 22, and 23. Simultaneous emission data for $\mathrm{Na}$ and $\mathrm{K}$ were acquired using pixels 159 and 459 respectively. Figures 20 and 22 show the sample-limited bandwidth spectra for $\mathrm{Na}$ and $\mathrm{K}$. Figures 21 and 23 show the $400 \mathrm{~cm}^{-1}$ immediately around the lines. The operating parameters for the spectra are listed in Tables 12 and 13.

In Figures 20 and 22, features (a) and (b) refer to the analyte lines. Features (c) appears to be $1 / \mathrm{f}$ detector noise. Since the amplitudes of (c) are both approximately 120 , an additive $1 / \mathrm{f}$ detector noise is indicated. Features (d) and (e) are indicated to be the base frequencies for 120 and $240 \mathrm{~Hz}$ multiplicative interferences for several reasons. First, the positions of (d) and (e) are at 120 and $240 \mathrm{~Hz}$ in both spectra. Second, their amplitudes are proportional to the total intensities of the analyte lines in the respective spectra.

The advantages of the multichannel/multiplex mode are as follows: Analyte signals are available for ratioing and comparison between channels, thus facilitating standard additions. Analyte signals are often separated from noise, with much noise distributed off of the analytical lines. Noises and interference are often separated from each other based upon noise type and distribution. Specific information on noise distributions and frequencies is often available, facilitating analysis of both noise sources and plasma processes.

\section{Spectrometric Performance Summary}

Table 14 is a summary of MMS performance data for the interferometric and multichannel/multiplex spectra in Figures 16, 19, and 20 to 23. Experimental and reference data for the $\mathrm{Na}$ and $\mathrm{K}$ lines are included. Intensity reference data is included for intensity comparisons 


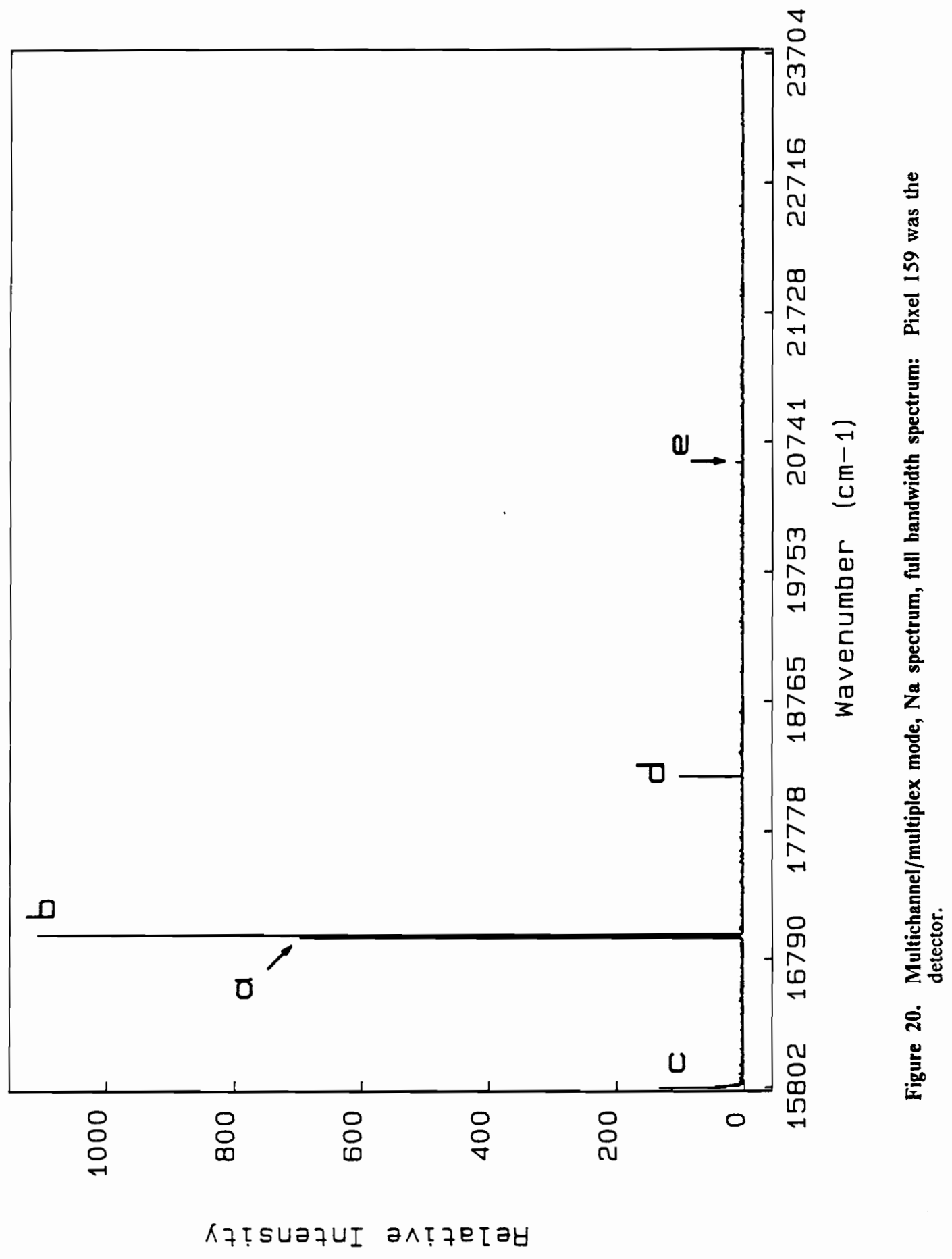




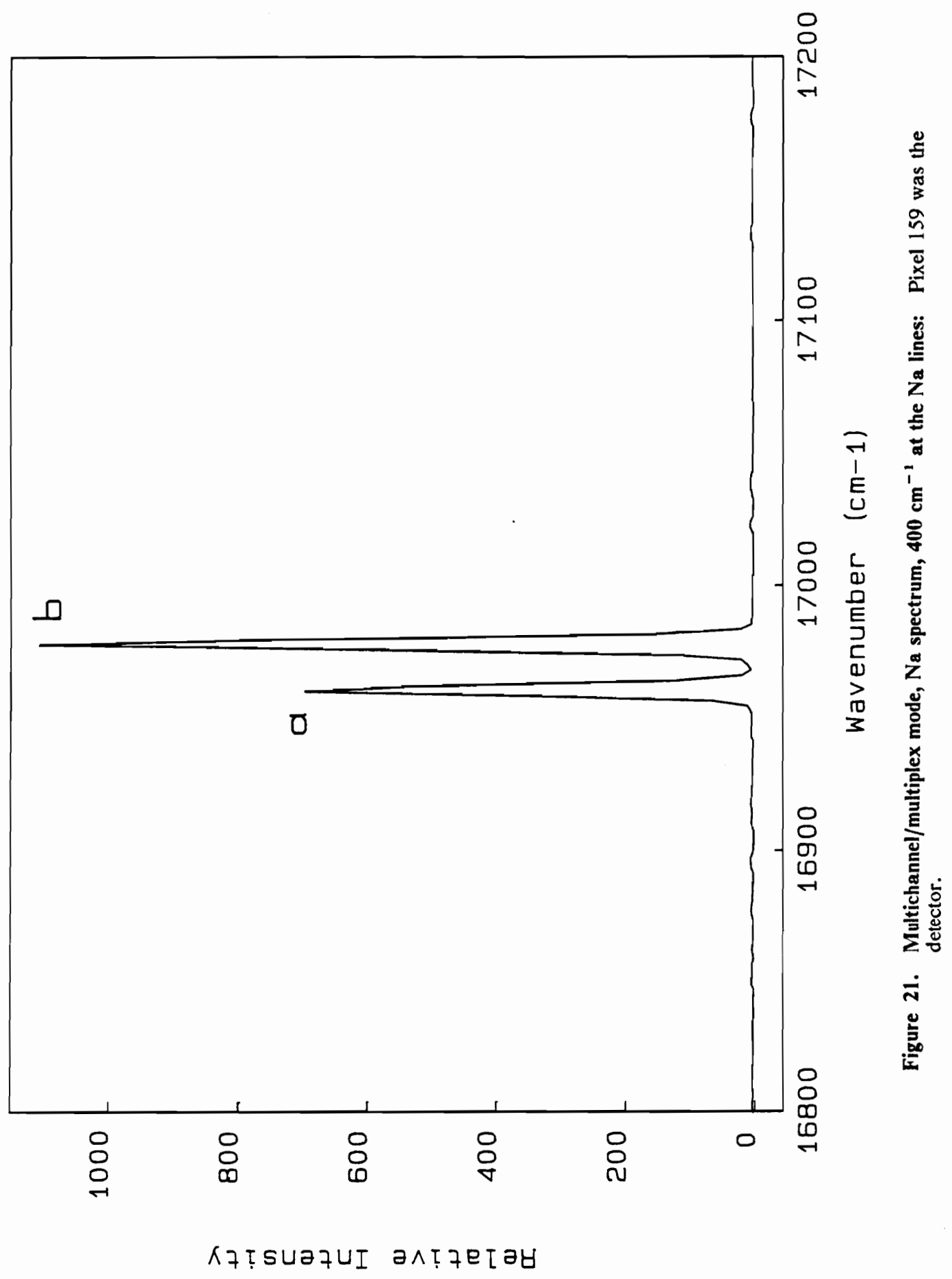




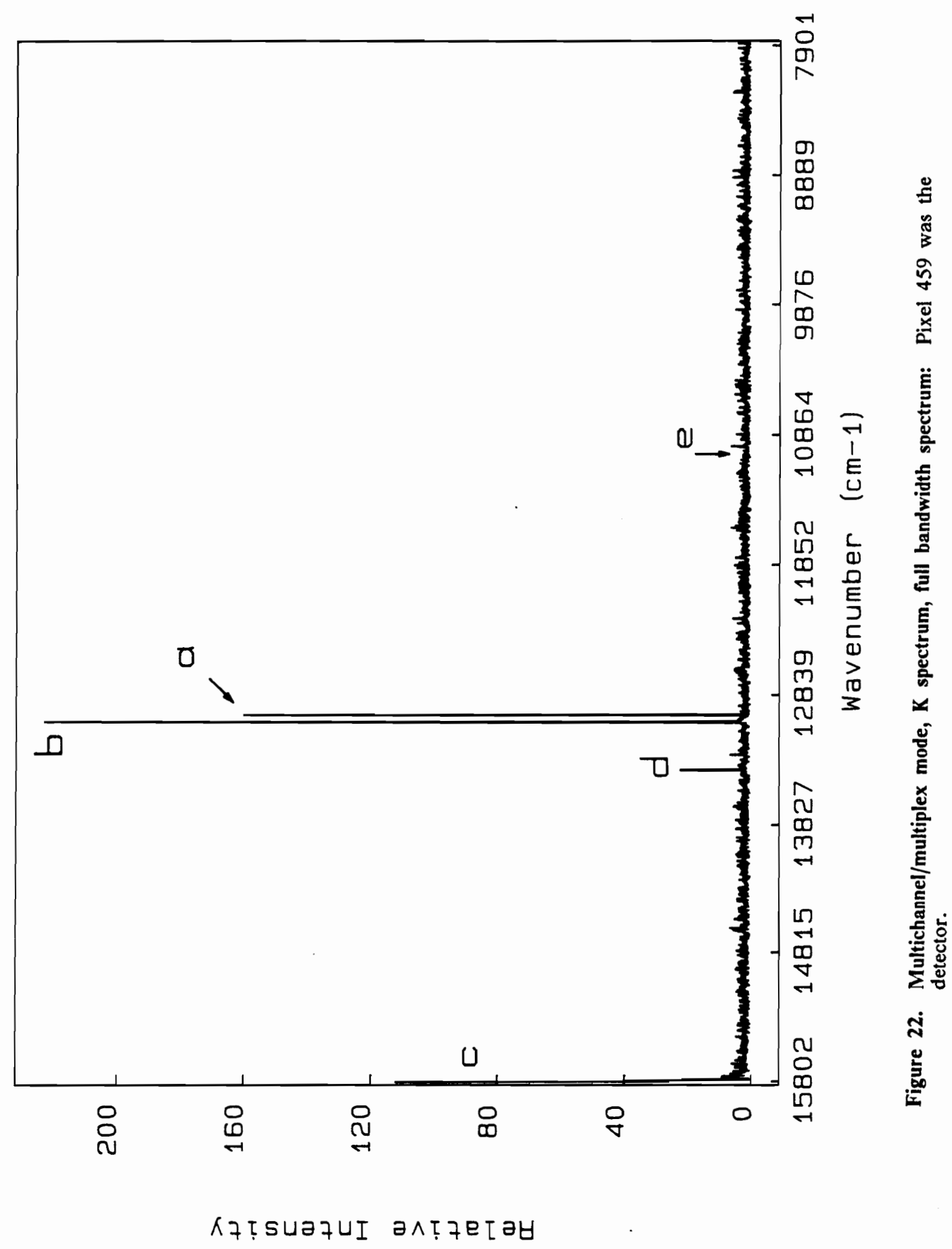




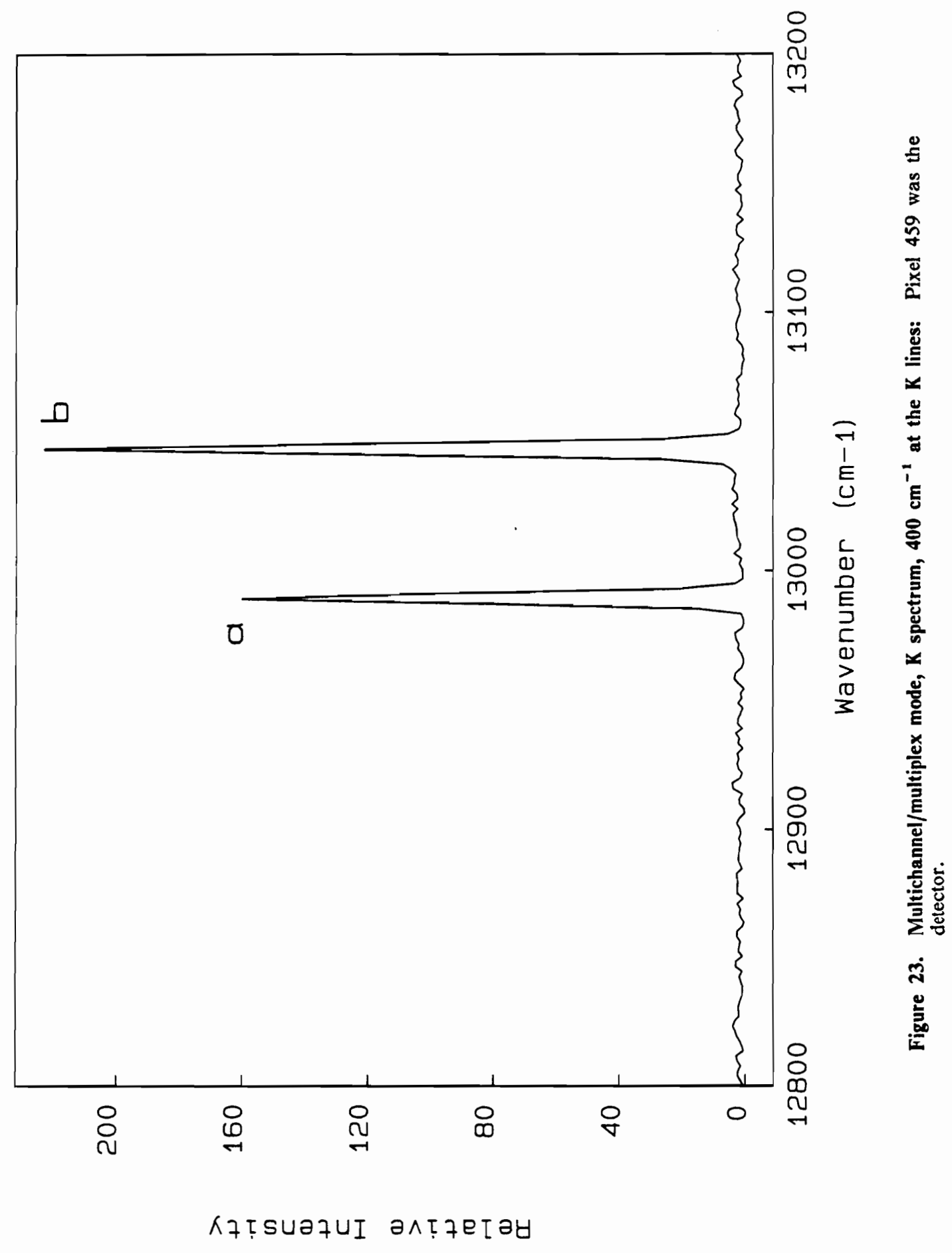


Table 12. Operating Parameters for Figures 20 and 21

Experimental parameters:

Source:

MIP Sample:

MIP

Mode:

$\mathrm{Na}, 100 \mu \mathrm{g} / \mathrm{mL}$

$\mathrm{K}, 100 \mu \mathrm{g} / \mathrm{mL}$

Interferometric

Transfer aperture:

Spectral bandwidth:

Pixel number:

$5.000 \times 0.250 \mathrm{~mm}$.

$6.0 \mathrm{~nm}$

Bandwidth center:

159

Alias factor:

Sampling bandwidth:

Number of points:

(per interferometer scan)

Resolution:

Resolving power:

$590 \mathrm{~nm} ; 16,950 \mathrm{~cm}^{-1}$

[3:4]

$7,901 \mathrm{~cm}^{-1}$

8,192 .

Coadded mirror scans:

$1.929 \mathrm{~cm}^{-1}$.

8,800

Total exposure time:

100

Total elapsed time:

$256 \mathrm{sec}(4 \mathrm{~min}, 16 \mathrm{sec})$

Apodization:

$55 \mathrm{~min}$.

Gaussian 
Table 13. Operating Parameters for Figures 22 and 23

Experimental parameters:

Source:

MIP Sample:

Mode:

Transfer aperture:

Spectral bandwidth:

Pixel number:

Bandwidth center:

Alias factor:

Sampling bandwidth:

Number of points:

(per interferometer scan)

Resolution:

Resolving power:

Coadded mirror scans:

MIP

$\mathrm{Na}, 100 \mu \mathrm{g} / \mathrm{mL}$

$\mathrm{K}, 100 \mu \mathrm{g} / \mathrm{mL}$

Interferometric

$5.000 \times 0.250 \mathrm{~mm}$.

$6.0 \mathrm{~nm}$

459

$768 \mathrm{~nm} ; 13,020 \mathrm{~cm}^{-1}$

[2:4]

$7,901 \mathrm{~cm}^{-1}$

8,192 .

Total exposure time:

Total elapsed time:

Apodization:

$1.929 \mathrm{~cm}^{-1}$.

6,750

100

$256 \mathrm{sec}(4 \mathrm{~min}, 16 \mathrm{sec})$

$55 \mathrm{~min}$.

Gaussian 
within a line pair only. Experimental line positions were taken as the points with the greatest intensities in the lines. This is not rigorously correct, but there is no line-finding program for the MMS at this time. The line position data shows excellent accuracy. Inherent wavenumber accuracy is a property of FTS, so a wavenumber calibration of the MMS was never required.

\section{Future Improvements to the MMS}

For several reasons, other solid-state detector technologies and architectures should be examined as the detector for the MMS. Other experiments have shown the limiting noise of the MMS to be PDA read noise. Read noise, an additive noise, is high in PDAs, ${ }^{9}$ and is notoriously high in PDAs operated with evaluation circuitry ${ }^{6,69}$ Improvements possible in this area include optimizing the detector electronics, investigating the use of low read noise detectors such as CCDs and CIDs, and detector cooling. Other detector technologies might also be useful in controlling the FT resolution aperture and the dispersive subsystem filter function. The vertical and horizontal binning capabilities in CCD's for example would be very useful in these two areas.

Examination of the operating parameter tables shows a large difference between the total exposure times and the total elapsed times in the interferometric and multichannel/multiplex modes. For example, the total exposure time in Table 9 is $256 \mathrm{sec}$, while the total elapsed time is $50 \mathrm{~min}$. Total exposure time is given by

$$
t_{E x}=t_{A p} \cdot N_{I n t} \cdot N_{S c}
$$

where $t_{E x}$ is the total exposure time, $t_{A p}$ is the aperture time for one array read $(0.3125 \mathrm{msec}), N_{I n t}$ is the number of points in one interferogram, and $N_{S c}$ is the number of coadded scans. Total exposure time is analogous to the integration time for systems with integrating detectors. In contrast, the total elapsed time is the complete time for data acquisition. The difference between these times is due to the overhead in the mirror movement cycle. The mirror drive and tracking 
Table 14. MMS Determination of MIP Line Parameters

\begin{tabular}{|c|c|c|c|c|c|}
\hline & $\begin{array}{l}\text { Wavenumber } \\
\left(\mathrm{cm}^{-1}\right) \text {, air }\end{array}$ & $\begin{array}{l}\text { Wavelength } \\
\text { (nm), air }\end{array}$ & Intensity & Noise & $\mathrm{S} / \mathrm{N}$ \\
\hline \multicolumn{6}{|c|}{ Literature values: ${ }^{a}$} \\
\hline Line a): & $16,960.873$ & 589.5923 & 5000 & -. & - \\
\hline Line b): & $16,978.064$ & 588.9953 & 9000 & -- & -- \\
\hline \multicolumn{6}{|l|}{$\mathrm{K}:$} \\
\hline Line a): & $12,988.735$ & 769.8979 & 5000 & -- & -- \\
\hline Line b): & $13,046.473$ & 766.4907 & 9000 & -- & -. \\
\hline \multirow{2}{*}{\multicolumn{6}{|c|}{$\begin{array}{l}\text { Experimental values: } \\
\text { Interferometric mode: }\end{array}$}} \\
\hline & & & & & \\
\hline \multicolumn{6}{|c|}{$\mathrm{Na}$ : } \\
\hline \multicolumn{6}{|l|}{ Fig. 12:b } \\
\hline $\begin{array}{l}\text { Line a): } \\
\text { error: }\end{array}$ & $\begin{array}{r}16,960.212 \\
-0.661\end{array}$ & $\begin{array}{r}589.6153 \\
+0.0230\end{array}$ & 1017.0 & 1.253 & 812 \\
\hline $\begin{array}{l}\text { Line b): } \\
\text { error: }\end{array}$ & $\begin{array}{r}16,977.573 \\
-0.491\end{array}$ & $\begin{array}{l}589.0123 \\
+0.0170\end{array}$ & 1687.1 & 1.253 & 1,346 \\
\hline \multicolumn{6}{|l|}{ Fig. 14:c } \\
\hline & $\begin{array}{r}16,961.176 \\
+0.303\end{array}$ & $\begin{array}{r}589.5818 \\
-0.0105\end{array}$ & 448.4 & 1.367 & 328 \\
\hline $\begin{array}{l}\text { Line b): } \\
\text { error: }\end{array}$ & $\begin{array}{r}16,978.055 \\
-0.009\end{array}$ & $\begin{array}{l}588.9956 \\
+0.0003\end{array}$ & 711.3 & 1.367 & 520 \\
\hline \multicolumn{6}{|c|}{ Multichannel/multiplex mode: } \\
\hline \multicolumn{6}{|c|}{$\mathrm{Na}$, Fig. $16^{b}$} \\
\hline $\begin{array}{l}\text { Line a): } \\
\text { error: }\end{array}$ & $\begin{array}{r}16,960.212 \\
-0.661\end{array}$ & $\begin{array}{l}589.6153 \\
+0.0230\end{array}$ & 697.3 & 1.980 & 352 \\
\hline $\begin{array}{l}\text { Line b): } \\
\text { error: }\end{array}$ & $\begin{array}{r}16,977.573 \\
-0.491\end{array}$ & $\begin{array}{l}589.0123 \\
+0.0170\end{array}$ & 1107.3 & 1.980 & 559 \\
\hline \multicolumn{6}{|l|}{ K, Fig. 17:d } \\
\hline $\begin{array}{l}\text { Line a): } \\
\text { error: }\end{array}$ & $\begin{array}{r}12,988.2967 \\
-0.4383\end{array}$ & $\begin{array}{r}769.9239 \\
0.0260\end{array}$ & 160.2 & 1.624 & 99 \\
\hline $\begin{array}{l}\text { Line b): } \\
\text { error: }\end{array}$ & $\begin{array}{r}13,046.168 \\
-0.305\end{array}$ & $\begin{array}{l}766.5089 \\
+0.0182\end{array}$ & 222.9 & 1.624 & 137 \\
\hline
\end{tabular}

- Literature values from references 67 and 68. Intensities are for arc sources.

$b, c, d$ Acquisition conditions:

\begin{tabular}{rcc} 
Alias & $\begin{array}{c}\text { Resolution } \\
\text { Interval (cm }\end{array}$ & $\begin{array}{c}\text { Resolution } \\
\text { Interval (nm) }\end{array}$ \\
\hline 4 & 1.929 & 0.0670 \\
16 & 0.4823 & 0.01675 \\
4 & 1.929 & 0.1138
\end{tabular}


system in this interferometer is relatively simple. Consequently, much time is spent moving the mirror between the end-of-scan reference position and the data collection region around the zero path difference. For example, Table 9 indicates that $>99 \%$ of the time was consumed with mirror movement overhead and $<1 \%$ was available for data collection. The use of a more sophisticated mirror tracking and drive system could drastically reduce the total elapsed time..$^{41,50}$ 


\section{Discussion}

The total S/N gain of a spectrometer design, $G_{T}$, is the product of the multiplex advantage (or disadvantage) and the throughput advantage. ${ }^{70}$ This is expressed by the equation

$$
G_{T}=G_{M} \cdot G_{\Omega}
$$

where $G_{M}$ is the multiplex advantage (or disadvantage), and $G_{\Omega}$ is the throughput advantage. ${ }^{70} G_{M}$ and $G_{\Omega}$ are both dependent upon the type of limiting noise however. The relevant types of noise for both $G_{M}$ and $G_{\Omega}$ are detector, photon, and source noise.

A primary goal of the MMS is to optimize $G_{T}$, the total $\mathrm{S} / \mathrm{N}$ gain, by trading some throughput for a reduction in noise. The purpose of the flexible filtering and the multichannel/multiplex mode are to reduce photon and source noises. The purpose of the optical design is to maximize throughput and signal. The throughput of the MMS cannot be as large as that of an interferometer, however, due to the need for slits for flexible filtering. The functions of slits in the MMS are provided by the transfer aperture and the pixel. The $\mathrm{S} / \mathrm{N}$ can be maximized by finding the correct compromise between throughput and noise reduction.

The effect of limiting noise on the multiplex advantage was discussed above. This section discusses the following: the effects of types of limiting noise on the throughput advantage, limiting noises in AES, the MMS's throughput, and the total S/N gain of the MMS. 


\section{The Throughput Advantage and Limiting Noises in AES}

The throughput advantage is the ability of one spectrometer to increase $\mathrm{S} / \mathrm{N}$ via an increase in throughput and signal relative to another spectrometer. The type of limiting noise is very important. Photon and source noise are dependent upon source intensity. Consequently, an increase in throughput does not always lead to a corresponding increase in $S / N$.

If detector noise is limiting, the noise is independent of the signal and is constant. In this case, the $\mathrm{S} / \mathrm{N}$ is directly proportional to the throughput, and the throughput advantage is directly proportional to any throughput increase. This situation is typical of the mid-IR and FT-IR.

If photon noise is limiting, the noise is proportional to the square root of the total detected photons. Therefore, the $\mathrm{S} / \mathrm{N}$ is proportional to the square root of the throughput, and the throughput advantage is proportional to the square root of any throughput increase. The ideal spectrometer, with perfect detector and source, is photon-noise limited. Photon-noise limited conditions are often met in the UV and visible because of good detector technology.

If source noise is limiting, the noise is proportional to the signal, and the $\mathrm{S} / \mathrm{N}$ is constant. Significantly, there is no throughput advantage because increasing throughput causes signal and noise to increase at the same rate. This again underscores the severity of source noise and the value of internal standards.

In AES, the limiting noise should range between photon and source noise. AES is often photon-noise limited if sample concentrations are near the detection limit. ${ }^{71}$ AES is often source-noise limited if sample concentrations are well above the detection limit (>100 times). ${ }^{11}$ Nebulizer and plasma fluctuations are often the origin of the source noise. Under photon- or source-noise limited conditions, the potential for an FTS throughput advantage is reduced or eliminated.16,50 The MMS, however, is specifically designed to minimize noise under these conditions and retain other FTS advantages. 


\section{Throughput and the Throughput Advantage of the MMS}

Table 15 compares the throughputs and throughput advantages for three spectrometers: a 1-m Czerny-Turner monochromator, the MMS, and a conventional interferometer. The comparisons are made for equal resolving powers and at two wavelengths, 590 and $300 \mathrm{~nm}$. 590 and $300 \mathrm{~nm}$ were chosen because emissions at $590 \mathrm{~nm}$ have been shown in this chapter and 300 $\mathrm{nm}$ is more typical of analytical atomic emissions.

The throughput of an optical system is defined as $^{72}$

$$
E=A \cdot \Omega
$$

where $E$ is the throughput in ( $\mu m^{2} \cdot$ steradians), $A$ is the area of the limiting field stop in $\mu m^{2}$, and $\Omega$ is the solid angle subtended by the limiting optic in steradians. Using the small angle approximation, ${ }^{73}$ the equation becomes

$$
E=\frac{A_{1} \cdot A_{2}}{l^{2}}
$$

where $A_{1}$ is the area of the limiting optic, or aperture stop; $A_{2}$ is the area of the limiting field stop; and $l$ is the distance between $A_{1}$ and $A_{2} A_{1}$ in a monochromator is usually the illuminated area of the grating; in an interferometer $A_{1}$ is usually the illuminated area of the beamsplitter. $A_{2}$ in a monochromator is usually the area of the entrance or exit slit, whichever is smaller; in an interferometer at high resolution $A_{2}$ is usually the area of the source or detector, again, whichever is smaller. Expressing throughput in terms of the $f$-number of the optics and the area of the field aperture, the equation becomes

$$
E=\frac{\pi \cdot A_{2}}{4(f / \#)^{2}}
$$

Table 15A summarizes the relevant optical parameters of the spectrometers for the throughput calculations. The parameters for $A_{2}$ were obtained by first assuming a resolution of $0.4823 \mathrm{~cm}^{-1}$, the highest attained by the MMS. The dimensions $A_{2}$ were then obtained by applying 
A: Optical Parameters:

- $f / \#$

- Field aperture limits:

Resolution: $0.4823 \mathrm{~cm}^{-1}$

$590 \mathrm{~nm}, \mathrm{R}: 35,200$

Monochromator and MMS:

slit height $(\mathrm{mm})$ :

slit width $(\mathrm{mm})$ :

Interferometer:

detector diameter $(\mathrm{mm})$ :

$300 \mathrm{~nm}, \mathrm{R}: 69,110$

Monochromator and MMS:

slit height $(\mathrm{mm})$ :

slit width $(\mathrm{mm})$ :

Interferometer:

detector diameter $(\mathrm{mm})$ :
Czerny-Turner

monochromator $^{a}$

$$
7.0
$$

MMS Interferometer ${ }^{b}$

3.0

3.0

B: Absolute Throughputs: ( $\mu \mathrm{m}^{2} \bullet$ steradian)

Wavelength

$590 \mathrm{~nm}$ :

$300 \mathrm{~nm}$ :

\begin{tabular}{ccc}
$E_{\text {Mono }}$ & $E_{\text {MMS }}$ & $E_{\text {Int }}$ \\
\hline 962 & 5,600 & 224,000 \\
250 & 3,900 & 114,000
\end{tabular}

\begin{tabular}{lccc} 
C: Throughput Ratios: & $E_{M M S}$ & $E_{l n t}$ & $E_{l n t}$ \\
\cline { 2 - 4 } & $E_{\text {Mono }}$ & $E_{M M S}$ & $E_{\text {Mono }}$ \\
\cline { 2 - 4 } $590 \mathrm{~nm}:$ & 5.8 & 40 & 233 \\
$300 \mathrm{~nm}:$ & 16 & 29 & 460 \\
\hline D: Throughput Advantages: & $E_{M M S}$ & $E_{l n t}$ & $\frac{E_{l n t}}{n}$ \\
\cline { 2 - 4 } & $E_{\text {Mono }}$ & $E_{M M S}$ & $E_{\text {Mono }}$ \\
\hline $90 \mathrm{~nm}:$ & 5.8 & 40 & 233 \\
Detector Noise Limited & 2.4 & 6.3 & 15.3 \\
Photon Noise Limited & 1 & 1 & 1 \\
Source Noise Limited & & & \\
300 nm: & 16 & 29 & 460 \\
Detector Noise Limited & 4 & 5.4 & 21 \\
Photon Noise Limited & 1 & 1 & 1 \\
Source Noise Limited & & 1 &
\end{tabular}

a Monochromator chosen for throughput comparisons: $1.0 \mathrm{~m}$ Czerny-Turner, McPherson model $2061 \mathrm{~A}, \mathrm{f} / \#: 7.0$, Grating: $1800 \mathrm{G} / \mathrm{mm}$; holographic, Range: 185-860 nm, Reciprocal linear dispersion: $0.555 \mathrm{~nm} / \mathrm{mm}$.

b The interferometer chosen is the interferometer within the MMS except the detector aperture is circular and it is at the normal location. 
the appropriate resolution criteria for the respective spectrometers, ${ }^{39,74,59}$ and then back-calculating to obtain the correct output aperture geometries. For the MMS the slit height is the height of the transfer aperture, Al, that is required to intercept the entire first interference ring from the interferometer at maximum optical path difference. The MMS slit width is the width of a single PDA pixel. It should be noted that the throughput of the MMS could be doubled if just two adjacent pixels could be "binned".

Table 15B lists the throughputs for the spectrometers calculated from the parameters in Part A. Table $15 \mathrm{C}$ lists throughput ratios at the two wavelengths. As shown by these Tables, the MMS has an optical throughput that is intermediate between the monochromator and the interferometer.

The throughput advantages versus different limiting noises are summarized in Table 15D. A "1" indicates no throughput advantage. Two trends are shown in this Table. First, the throughput advantage of the interferometer and the MMS over the monochromator increases as wavelength decreases. Consequently, any throughput advantage becomes increasingly more significant toward the short wavelength limit of AES. Second, as the limiting noise progresses from detector to photon to source noise, the throughput advantage gradually disappears. Increases in throughput become less important as the limiting noise approaches source noise.

\section{Total S/N Gain of the MMS}

As stated above, the limiting noise for AES should range between photon and source noise. It is therefore useful to examine the throughput advantage and the total $\mathrm{S} / \mathrm{N}$ gain of the MMS under these two conditions. This does assume that detector noise in the MMS can be reduced to insignificant levels through the detector system improvements discussed above.

If source noise is limiting, there is no throughput advantage for any spectrometer. The total $\mathrm{S} / \mathrm{N}$ gain or loss for the MMS is then entirely dependent upon the multiplex term. For FTS and 
the MMS, a multiplex advantage or disadvantage is difficult to predict however, since the precise effects of redistributed source noises depends upon the noise distributions. Several generalizations can be made concerning source noise. First, every effort should be made to minimize source noise in the emission source. Second, emission lines should be spectrally bandlimited to reduce the effects on other, particularly weaker, emission lines. Third, internal standards should be implemented to compensate for residual effects of the source noise. The MMS addresses the last two points through the multichannel/multiplex mode.

If photon noise is limiting, the total $\mathrm{S} / \mathrm{N}$ gain or loss is a balance between the throughput gain and the multiplex gain. Throughput gain is addressed first. Comparing the MMS to the monochromator at $590 \mathrm{~nm}$, the MMS has a 5.8 times greater throughput, and should have a throughput advantage of $\sqrt{5.8}$, or 2.4. Comparing the MMS to the interferometer at $590 \mathrm{~nm}$, the interferometer has a 40 times greater throughput than the MMS, and should have a throughput advantage of 6.3 .

The multiplex gain is best done by example. The central column of Table 15D provides a useful example of the impact of the MMS's flexible filtering on the multiplex disadvantage. If the MMS can exclude 40 lines with intensities equal to the analyte line, redistributed photon noise is reduced and the $\mathrm{S} / \mathrm{N}$ at the analyte line is improved by a factor of $\sqrt{40}$, or 6.3 . At this point, the MMS's noise reduction exactly compensates for the interferometer's increased throughput, and the MMS and the interferometer have equal $S / N$ gains. The same result is obtained if the MMS excludes only one line with 40 times the intensity of the analyte line. The exclusion of more lines, more intense lines, or the background continuum continues to improve the total $S / N$ gain in favor of the MMS versus the interferometer. This gain is due to the MMS spectral filtering, and is in spite of its reduced throughput.

Compared to the Czerny-Turner monochromator, the MMS has a consistent throughput advantage. The MMS's multiplex advantage depends upon the structure of the spectrum, the ability of the MMS filtering function to isolate the line of interest, and the ability to reject superfluous features. Also, much of the MMS's S/N advantage here relies on the ability of the 
Fourier transform process to redistribute noise from inside the optical filter bandpass and into the filter cutoff regions.

\section{MMS Dispersive Mode Throughput}

In the MMS dispersive mode, the interferometer reduces throughput by approximately $50 \%$ versus a normal PDA-based multichannel spectrometer. In the present MMS implementation, $50 \%$ of the source intensity is returned to the source by the interferometer during dispersive mode operation. Using an interferometer with servo control ${ }^{50}$ and the ability to hold the mirror at zero path difference would alleviate this problem. With this type of interferometer, the moving mirror could be parked at the zero path difference (ZPD), causing all of the source intensity to be transferred to the detector, and the spatial and temporal integration by the PDA would not be required. 


\section{Conclusion}

In this research, the feasibility of the MMS and its ability to operate in the dispersive, interferometric, and multichannel/multiplex modes have been demonstrated. In these modes, the MMS can simultaneously obtain signal and background data. In the interferometric mode, the feasibility of flexible optical filtering has been shown. Capabilities in noise rejection and noise analysis have been illustrated. The ability of the MMS to obtain data under controlled aliasing, and to use this capability for data compression has also been shown. For the multichannel/multiplex mode, the applicability of internal standards to source noise reduction has been discussed. Finally, the potential of flexible optical filtering to improve the $S / N$ in the spectrum, in spite of a reduced throughput, has been discussed.

The MMS is a prototype spectrometer, built to test the feasibility of the concepts behind the instrument. Due to the capabilities shown by the MMS, it appears that hybrid instruments like the MMS will be increasingly important in future developments in optical spectrometry. This is particularly true for very demanding areas such atomic emission spectrometry. 
MMS Probe of the MIP 


\section{Introduction:}

In atomic spectrometry, improvements and innovations continue in source and spectrometer development. New sources such as FANES, Furnace Atomic Nonthermal Excitation Spectrometry ${ }^{75-77}$ and FAPES, Furnace Atomization Plasma Emission Spectrometry, ${ }^{78-80}$ are being developed. These particular sources combine the capabilities of graphite furnace atomic absorption for low detection limits and micro sample volumes, with the capabilities of inductively-coupled plasma (ICP) for wide dynamic range and simultaneous analysis. Improvements in existing sources, such as laminar flow ICP ${ }^{81-84}$ and microwave-induced plasma (MIP) ${ }^{85,86}$ torches, and low-flow MIP ${ }^{86}$ and ICP ${ }^{84,82}$ torches have been reported. Investigations into helium ICPs ${ }^{84}$ and $\mathrm{MIPs}^{87}$ continue. Improvements in nebulizer technology also continue. ${ }^{88,89,90}$

Changes and improvements in source technology require that the operating conditions of the source be optimized. ${ }^{91}$ In addition, the implications of the source/spectrometer interactions must be explored. The ramifications of source viewing geometry and the optically-sampled source volume must be investigated.

Innovations in spectrometer design continue, $, 22,13,93,36$ permitting the acquisition of the needed source information. The new spectrometers are capable of extracting more spectral, spatial, and temporal data from new and existing emission sources than is possible with conventional systems. 
The purpose of this section is to utilize the Multi-Mode Spectrometer (MMS) to optimize the MIP, to provide a qualitative overview of the MIP source, to probe its emission characteristics, and to explore the MIP/MMS optical interaction. A cross-section of the MIP apparatus used is shown in Figure 24. Details of the observed emission zones within the MIP torch and cavity area are shown in Figure 25. Both end- on and side-on views of the plasma are shown. Placement of the MIP within the optical plan of the MMS is shown in Figure 26 for both side- on and end-on observation geometries.

A major emphasis of these experiments is to show that the low-resolution dispersive mode of the MMS can be used to rapidly obtain a substantial amount of information about a source. The MMS could be used to rapidly probe other atomic emission sources, or other types of sources such as Raman samples.

Prior to data acquisition, the MMS was used to optimize both the MIP operating parameters and the placement of the MIP in the focal plane of the MMS. The ability to simultaneously observe all emission lines, and to observe them in real time were distinct advantages in optimizing the MIP.

In these experiments, information was sought on the best observation geometry of the MIP. The relative advantages of side-on versus end- on optical viewing of the plasma was investigated. Previous studies of ICPs by Faires and co-workers ${ }^{94}$ and Demers ${ }^{95}$ had indicated an effect of viewing height on sample and background intensities, and a possible advantage to end-on viewing. Previous studies of MIPs by Long and Perkins ${ }^{62}$ had indicated that intensity is a very strong function of viewing height. In addition, the observable structure of the MIP plasma and its small size suggested that an optimal viewing geometry must be investigated. Subsequent studies of MIPs by Perkins and Long ${ }^{87}$ have shown strong longitudinal and radial intensity variations in the plasma. The previous studies of the ICPs raised questions concerning signal intensity, noise levels, and self-absorption. ${ }^{94,95}$ For these experiments, a horizontal plasma orientation seemed most appropriate. This orientation allowed the emission region at the base of the plasma to be vertical, which made the plasma base parallel to the transfer aperture of the MMS. However, the horizontal plasma position prompted concerns over plasma distortion, stability, and symmetry. 


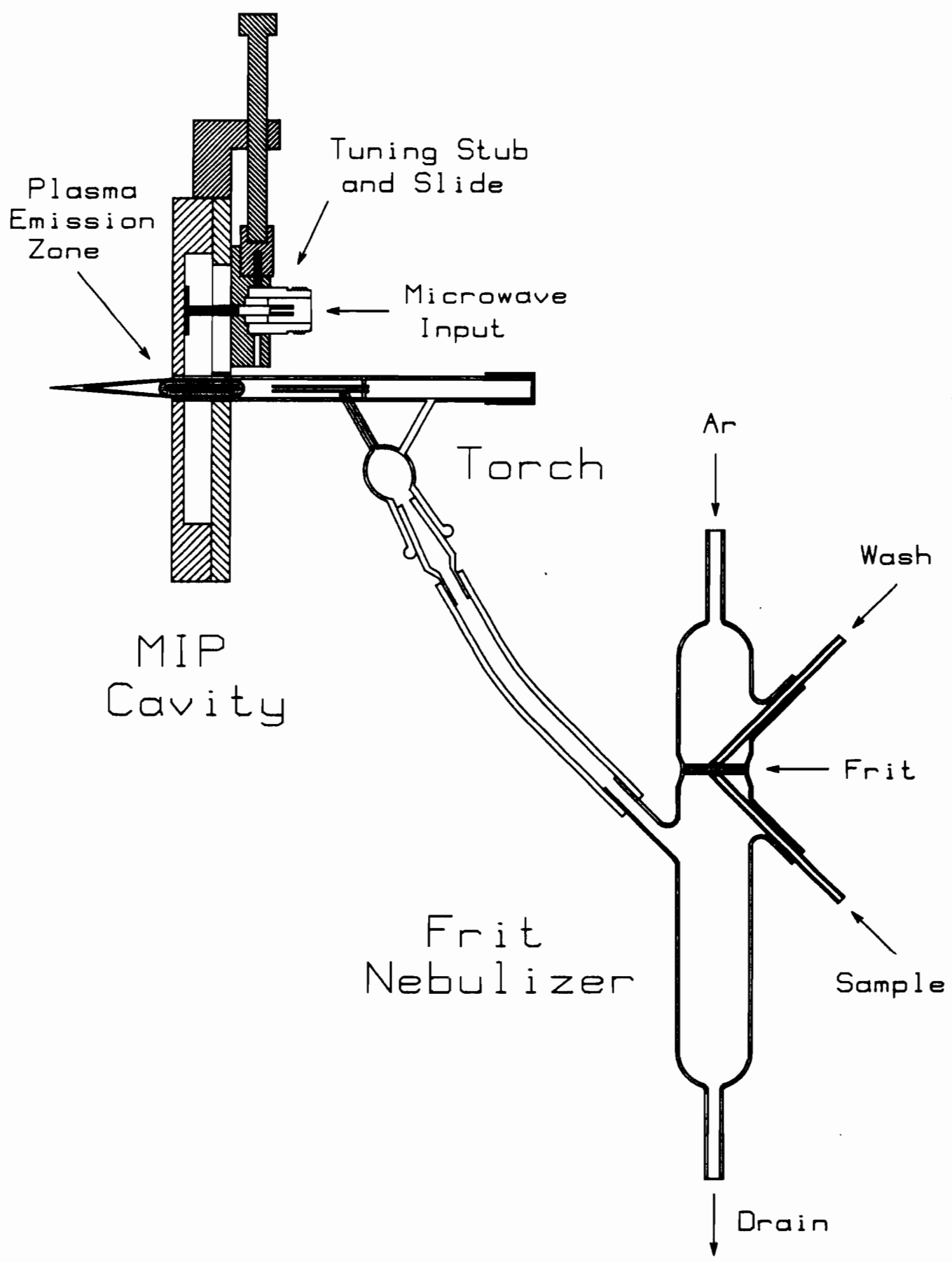

Figure 24. Cross-section of the MIP apparatus: The cavity, torch, and nebulizer are shown. 


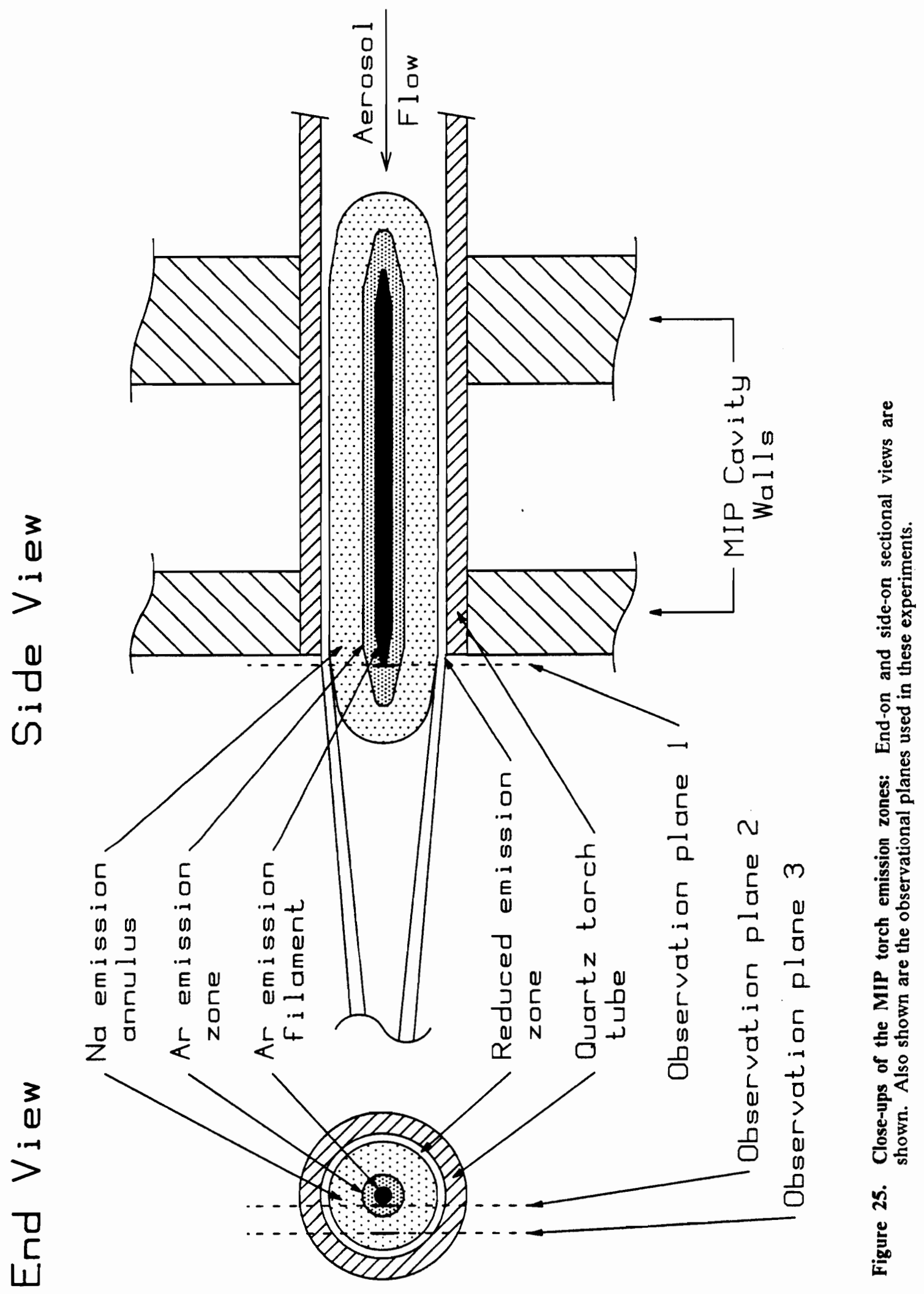



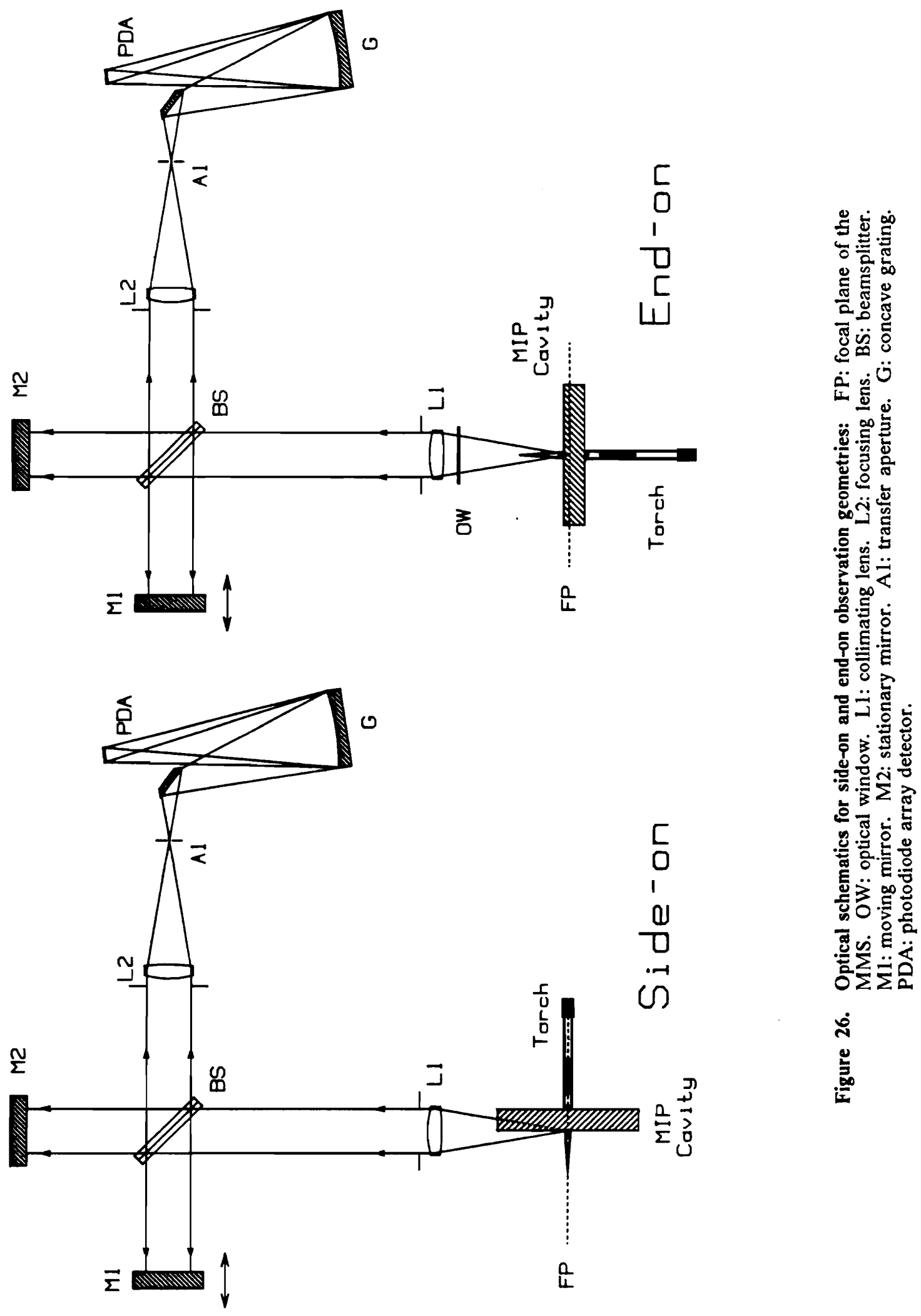

: 


\section{Experimental}

The equipment used in the MIP source are summarized in Table 16. The common MIP operating parameters are summarized in Table 17. Under these conditions, the plasma extended approximately $1 \mathrm{~cm}$ beyond the cavity. The $\mathrm{NaCl}$ and $\mathrm{KCl}$ salts used were reagent grade. Stock $1000 \mu \mathrm{g} / \mathrm{mL}$ solutions were prepared using distilled/deionizeld water. Other solutions were prepared by dilution using distilled/deionized water. 
Table 16. Equipment for the MIP

MIP Source and System:

Cavity:

Torch:

Nebulizer:

Microwave Generator:

Manufacturer:

Model:

Power:

Frequency:

Load:

High-efficiency $\mathrm{TM}_{010}$, Laboratory built Tangential flow, Laboratory built

Fritted disk, Laboratory built

Kiva Instrument Corp., Rockville, MD

MPG-4M

$120 \mathrm{~W}$

$2.450 \mathrm{GHz}$

$50 \mathrm{ohm}$

Sample Pump:

Manufacturer:

Type:

Model:

Gilson Medical Electronics, Middleton, WI

Peristaltic, 10 roller, 4 channel, variable speed.

Minipuls 2 
Table 17. MIP Operating Conditions

\section{Plasma Parameters:}

Plasma support gas:

Gas flow:

Nebulizer pressure:

Solution delivery rate:

Forward power:

Reflected power:

Ar

$2 \mathrm{~L} / \mathrm{min}$

$30 \mathrm{psi}$

$0.100 \mathrm{~mL} / \mathrm{min}$

$70 \mathrm{~W}$

$\sim 0 \mathrm{~W}$ 


\section{Results and Discussion:}

\section{Plasma Ignition and Optimization.}

Prior to operation, the plasma was ignited using the following procedure. The microwave power supply was allowed to warm up for about $10 \mathrm{~min}$. The nebulizer frit was rinsed several times with $10 \% \mathrm{HNO}_{3}$, followed by several rinses of deionized (DI) water. A sample flow of approximately $0.8 \mathrm{~mL} / \mathrm{min}$ of DI water was started onto the nebulizer frit. An argon flow of about $3 \mathrm{~L} / \mathrm{min}$ was established through the nebulizer. The microwave supply high-voltage section was turned on and the forward power set for $100 \mathrm{~W}$. The plasma was ignited with a Tesla coil. If necessary, the torch was pushed forward into the cavity until a proper laminar gas flow was established in the torch, and the plasma became centered. The torch tip was then made flush with the cavity face. The forward power was reduced to about $70 \mathrm{~W}$.

In the first optimization step, the MIP operation was addressed. The objectives were to maximize power transfer to the microwave cavity, minimize reflected power to the microwave power supply, and to minimize visible fluctuations in the plasma. A sample flow of $0.8 \mathrm{~mL} / \mathrm{min}$ of $\mathrm{Na}(500 \mu \mathrm{g} / \mathrm{mL})$ and $\mathrm{K}(500 \mu \mathrm{g} / \mathrm{mL})$ was pumped into the nebulizer. Iterative radial adjustments in the quartz tuning rod and the disk electrode were made until the reflected power was minimized. 
This also maximized power transfer to the cavity. Sample and argon flows were adjusted to assure continuous aerosol flow and to reduce visual plasma flicker. The MMS was not used in this initial step.

The second major optimization step involved placing the MIP in the focal plane of the MMS. The objective was to maximize detected emissions by the MMS. The MMS was used for fine position adjustment. Again, a sample solution contain $\mathrm{Na}$ and $\mathrm{K}$ was pumped onto the nebulizer frit. The MIP was placed in the side-on configuration near the focal point of the MMS entrance optics. See Figure 26. Adjustments in the gross position of the cavity and torch were made until a sharp image of the emission zones was visible on the slit of the transfer aperture, A1. Fine adjustments in the lateral position of the MIP were made along the focal plane (FP) of the MMS until the detected signal was maximized. This was done by setting the MMS up for free-running dispersive mode operation and monitoring the detected emission intensity of the plasma on the oscilloscope. The output of the photodiode array (PDA) was viewed in real time at $30 \mathrm{scans} / \mathrm{sec}$. Small adjustments of the MIP position were made until the emission intensities were maximized.

Finally, the detected signal was optimized by iteratively adjusting the MIP operation and the MIP placement, and monitoring the process with the MMS. Again, the objectives were to maximize detected emissions and to minimize source fluctuations. This was done by adjusting either the argon flow rate or the sample flow rate, noting the effect on detected intensity, and then cycling through optimizing the cavity and MIP placement. The Ar flow rate adjustment is a good example. Reducing the Ar flow from the initial setting produced two effects: an increase in the detected $\mathrm{Na}$ and $\mathrm{K}$ intensities due to increased sample residence time in the plasma; and a reduction in source fluctuation due to reduced sputter at the nebulizer frit. A change in argon flow, however, necessitated checking the tuning of the cavity. A reduced Ar flow also caused the emission zone to shift closer to the cavity face. This required repositioning the MIP in the focal plane. Reducing the Ar flow too much caused the plasma to become unstable, however, and also caused the maximum emission zone to move inside of the cavity face. 
Several iterations of the optimization steps resulted in an argon flow rate of $2 \mathrm{~L} / \mathrm{min}$, a sample flow rate of $0.100 \mathrm{~mL} / \mathrm{min}$, and a side-on observation geometry along observation plane 1 in Figure 25. When the plasma was fully optimized, the plasma appeared to be very stable and symmetrical, with no flicker or distortion.

\section{Side-on versus End-on Optical Throughput.}

It should be noted that in the side-on configuration, the MIP cavity blocks one half of the solid viewing angle of the MMS. This probably reduced detected intensities, but for several reasons it did not cause problems with the experiments. First, the optics of the MMS are symmetrical along the vertical plane parallel to the MIP cavity face. Therefore, the bisected optical system should be expected to perform essentially as the symmetrical optical system performed, but with one-half of the throughput. Second, the face of the cavity was polished, causing emissions radiated into the blocked half-angle to be reflected into the open half-angle.

\section{Side-on Emission Zone Dimensions.}

Measurements of the emission zone heights above the cavity and the longitudinal thickness of the $\mathrm{K}$ and $\mathrm{Na}$ emission zones were made in the side-on configuration along observation plane 1. These measurements were done by directly observing the dispersive mode output on the oscilloscope. Often, use could be made of the PDA's excellent geometric accuracy by using the $0.025 \mathrm{~mm}$ pixel-to-pixel spacing as a ruling increment. 
The optimal viewing height was measured by first optimizing the MIP/MMS interaction, described above. The viewing height then was determined by measuring the distance between the center of the transfer aperture and the image of the torch tip (1:1 magnification) projected onto the transfer aperture. The imaging ability of the MMS optical system was very good, and this measurement was not difficult. The optimal viewing height was found to be approximately 0.5 mm. This dimension is in agreement with measurements done by Long and Perkins ${ }^{62}$ using similar apparatus and conditions.

The longitudinal thickness of the zone of maximum $\mathrm{Na}$ emission was found to be approximately $0.35 \mathrm{~mm}$. This dimension was gauged in the following manner. The $0.250 \mathrm{~mm}$ wide transfer aperture caused a $0.250 \mathrm{~mm}$ slice ( $1: 1$ image) of the $\mathrm{Na}$ emission zone to be projected onto the PDA. If the emission intensity within this slice was uniform, the image detected by the PDA was a uniform trapezoid $0.250 \mathrm{~mm}$ (10 pixels) wide. If the $\mathrm{Na}$ emission intensity within this plasma slice was not uniform, the top of the trapezoid would droop correspondingly. When the MIP was in the optimum position, the trapezoid was uniform. When the MIP was translated to either side by approximately $0.05 \mathrm{~mm}$ ( 2 pixels), an edge of the trapezoid to began to droop. Therefore, the thickness of the optimum zone was approximately $0.35 \mathrm{~mm}$. Furthermore, translating the MIP approximately $0.25 \mathrm{~mm}$, or 1 slit width, to either side caused the top of the trapezoid to slope in a linear fashion from $100 \%$ to approximately $30 \%$ across the $0.250 \mathrm{~mm}$ observed height range. This indicated a $\mathrm{Na}$ emission zone approximately $0.65 \mathrm{~mm}$ thick at full- width half-maximum (FWHM).

The thickness of the $\mathrm{K}$ emission zone appeared to be considerably less than that for Na. When the $0.250 \mathrm{~mm}$ wide transfer aperture was used, the profile detected by the PDA was not the expected uniform trapezoid, but was an image displaying hyper-resolution. The image was a pair of lines with the spacing of the $\mathrm{K}$ doublet, but with a detected widths of about 4 pixels, or $0.1 \mathrm{~mm}$. The resolution was similar to that seen with the $0.100 \mathrm{~mm}$ wide transfer aperture. This hyper-resolution can be explained only by assuming that a false aperture had been inserted into the optical system at one of the focal planes. A false aperture could have been created by a $\mathrm{K}$ emission zone which was only about $0.1 \mathrm{~mm}$ thick. Therefore, the $\mathrm{K}$ emission was assumed to be only 0.1 mm thick. 


\section{Basic Plasma Emissions, Side-on Observation}

One purpose of these experiments was to identify the emission lines due to the support gas (Ar), the solvent $\left(\mathrm{H}_{2} \mathrm{O}\right)$, and the samples $(\mathrm{Na}$ and $\mathrm{K})$, and also to identify the spectral regions not obstructed by these lines. The MMS in the dispersive mode was used to rapidly identify the major emission lines of the MIP. Dispersive mode survey spectra of the MIP are shown in Figures 27 through 32. Dispersive mode difference spectra are shown in Figures 33 through 37 . The experimental parameters are listed in Table 18. To obtain these spectra, the MIP was set up in the side-on configuration.

The survey spectra in Figures 27 to 32 show the plasma emissions due to a number of species over the wavelength range 500 to $800 \mathrm{~nm}$. The spectra have equal vertical scales to facilitate comparison between spectra. Species introduced into the plasma for each run are summarized in Table 19. Ten spectra were coadded for each recorded spectrum. For Figure 27 the term "dry" frit means that the glass frit was made as dry as practical. Drying was done following the frit washing procedure by blowing dry Ar through the nebulizer for several minutes. Because totally dry Ar caused the plasma to become unstable, and because of the frit's large capacity for holding water, complete drying of the frit was not practical.

The difference spectra shown in Figures 33 to 37 are more revealing. These spectra were obtained from the survey spectra in Figures 27 to 32 through pertinent background corrections. Equal vertical scales are provided for comparison. It was these spectra which allowed the rapid identification of the plasma lines. Table 20 lists the emitting species for these Figures. Negative peaks in the spectra indicate plasma loading due to redistribution of the energy in the MIP. Figure 33, the Ar-corrected spectrum for DI water, readily shows the location of the $\mathrm{H}$ and $\mathrm{O}$ emissions. Using this spectrum, the remaining lines in Figure 28 can be assigned to Ar. Figures 34 through 37 show the background-corrected spectra, the emission spectra minus the $\mathrm{Ar}$ and $\mathrm{H}_{2} \mathrm{O}$ lines, for the sample spectra. 
Table 18. Experimental Parameters for the Emission Survey Spectra

Experimental parameters:

MIP parameters:

Viewing geometry:

Side-on

Observation plane 1,

$0.5 \mathrm{~mm}$ from cavity face.

MMS operating parameters:

Mode:

Spectral Range:

Spectral bandwidth:

Points per Array Scan:

Transfer aperture:

Resolution:

Dispersive

$500-800 \mathrm{~nm}$.

$300 \mathrm{~nm}$

512

$1.000 \times 0.050 \mathrm{~mm}$.

Average resolving power:

$1.2 \mathrm{~nm}$

Integration time:

540

Coadded scans per spectrum:

$33.3 \mathrm{msec}$

Total exposure time

per spectrum:

10

333 msec. 


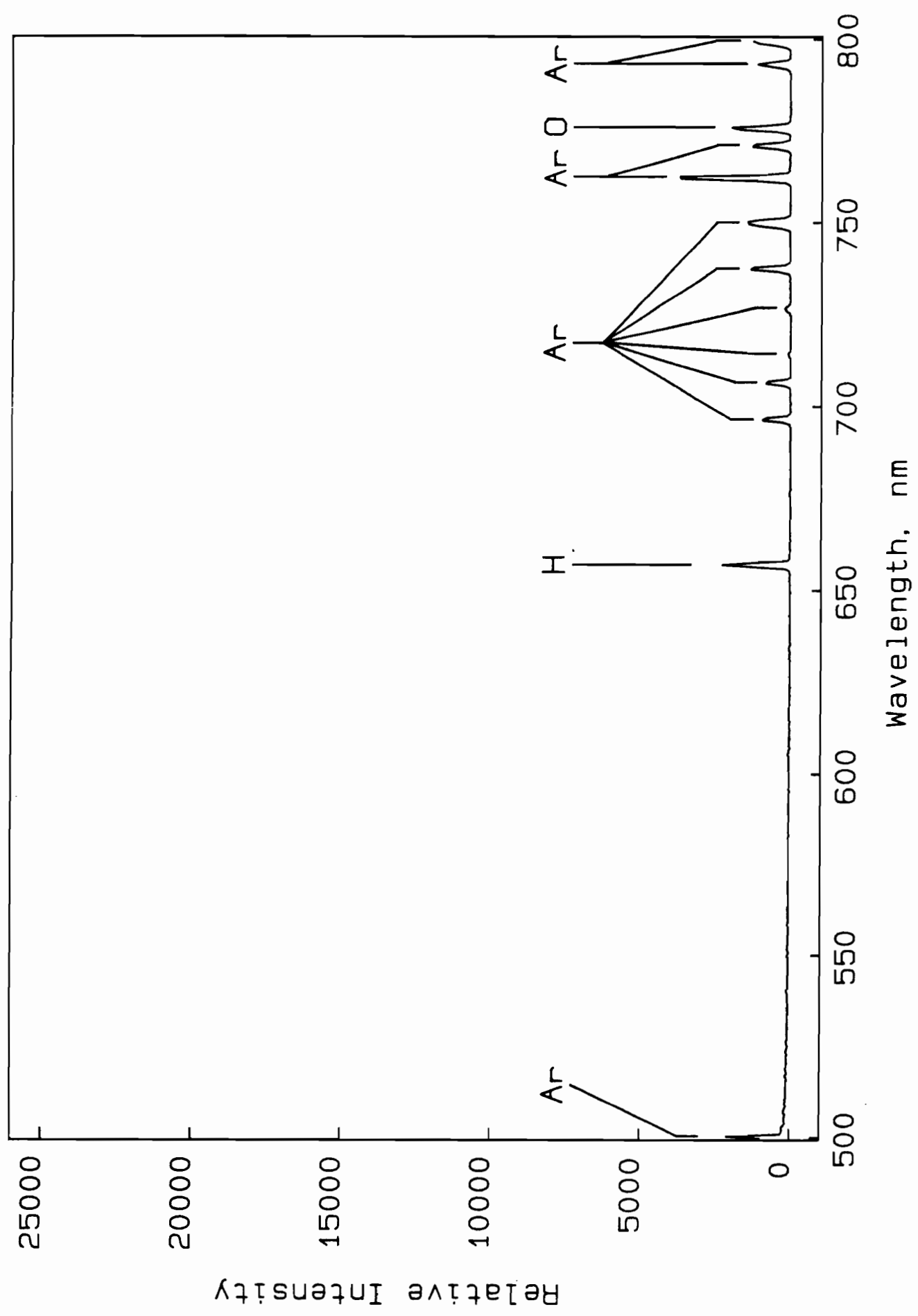

是 


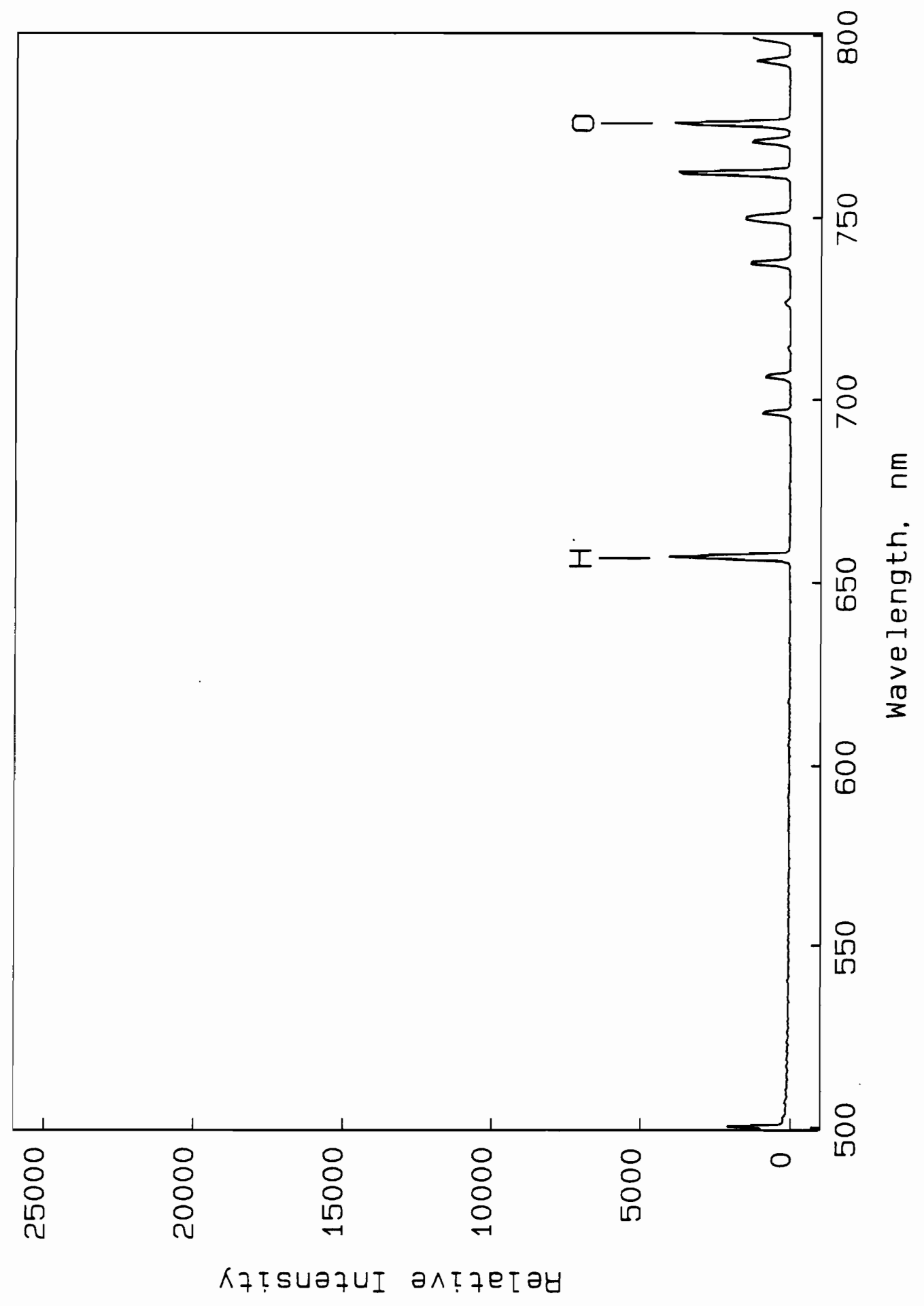

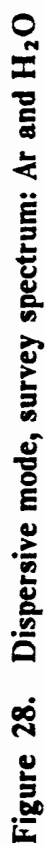




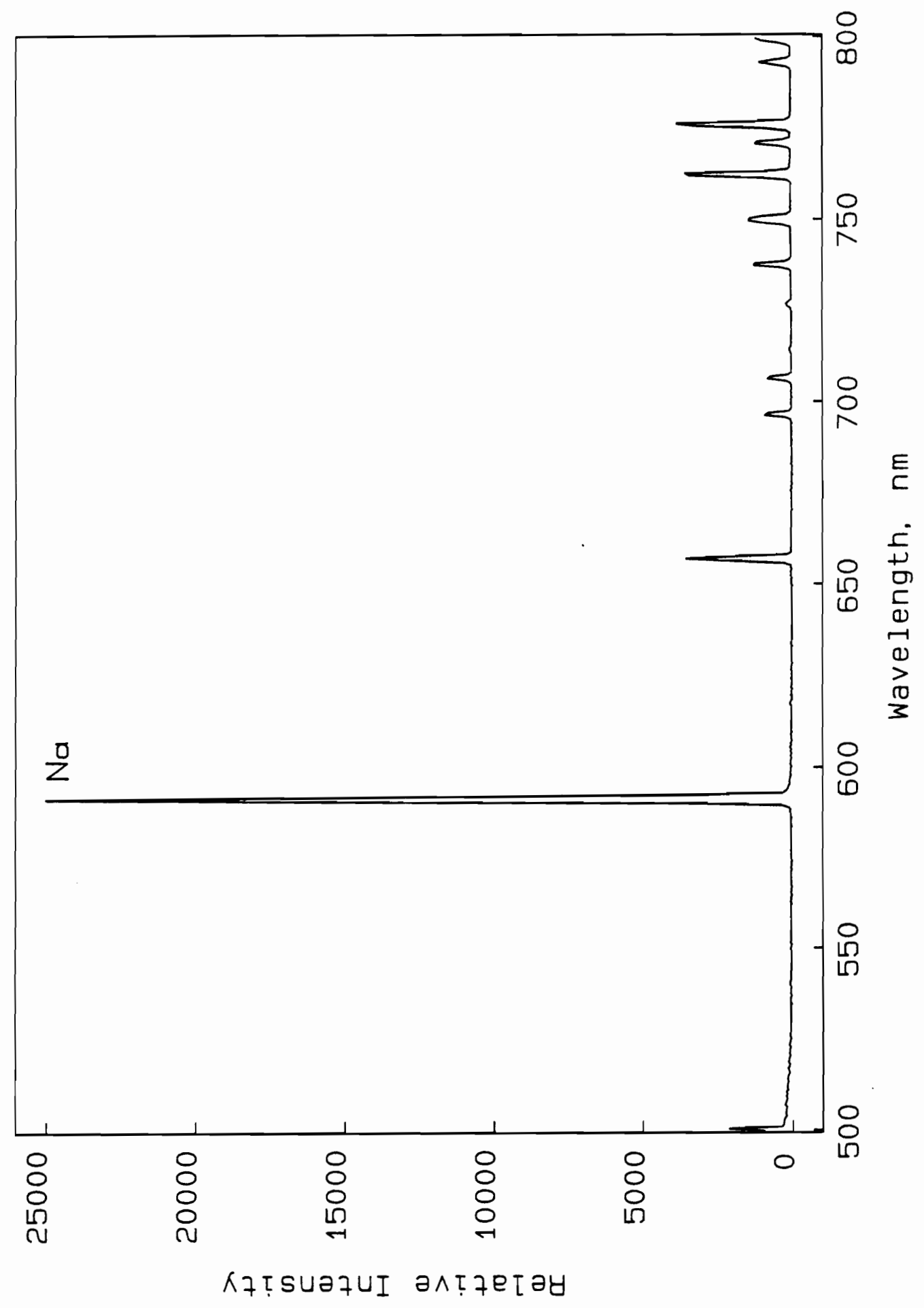

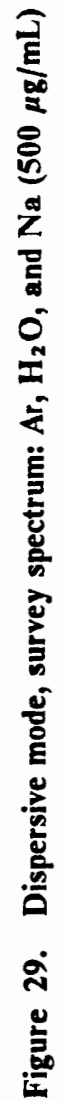




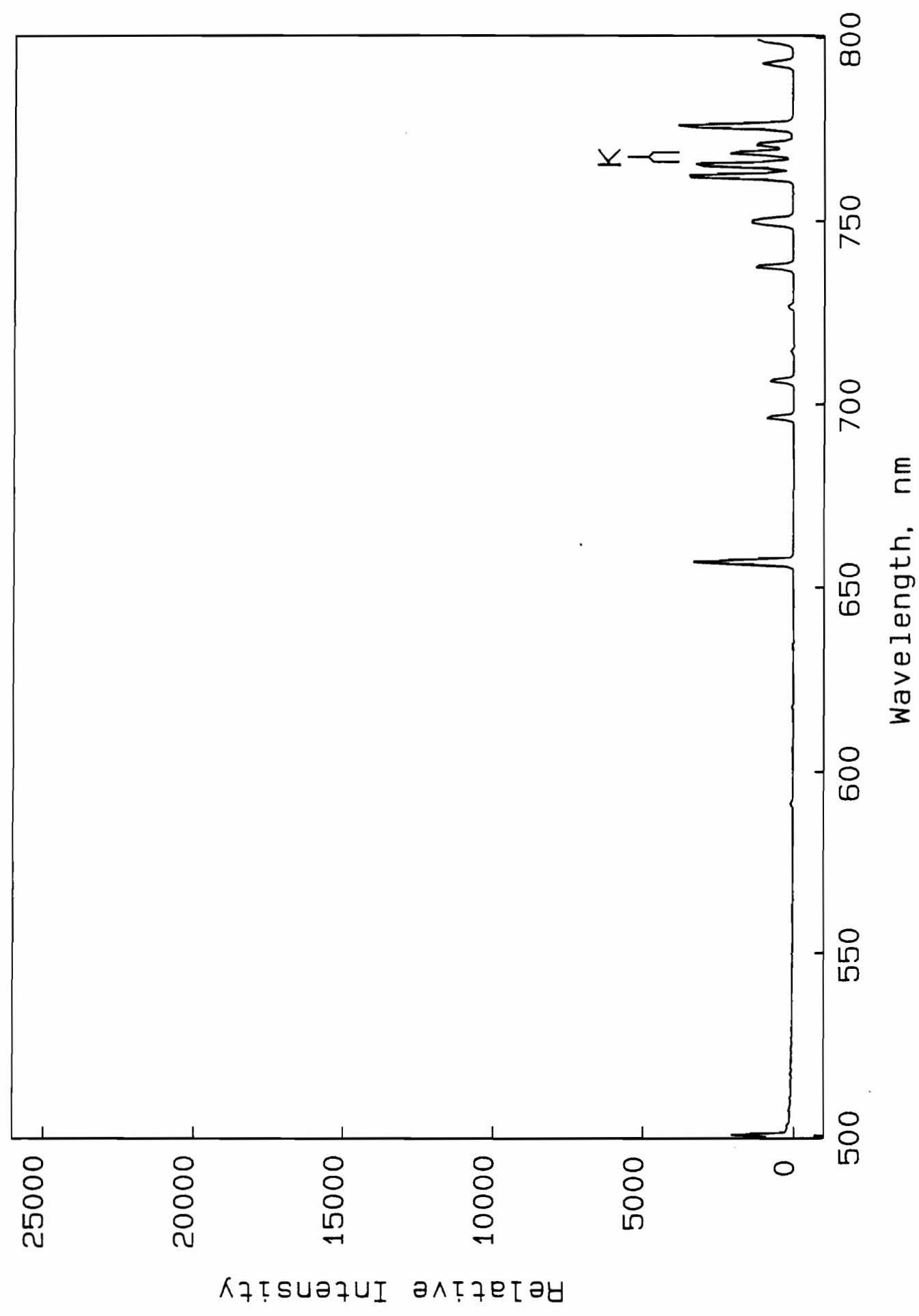

定 


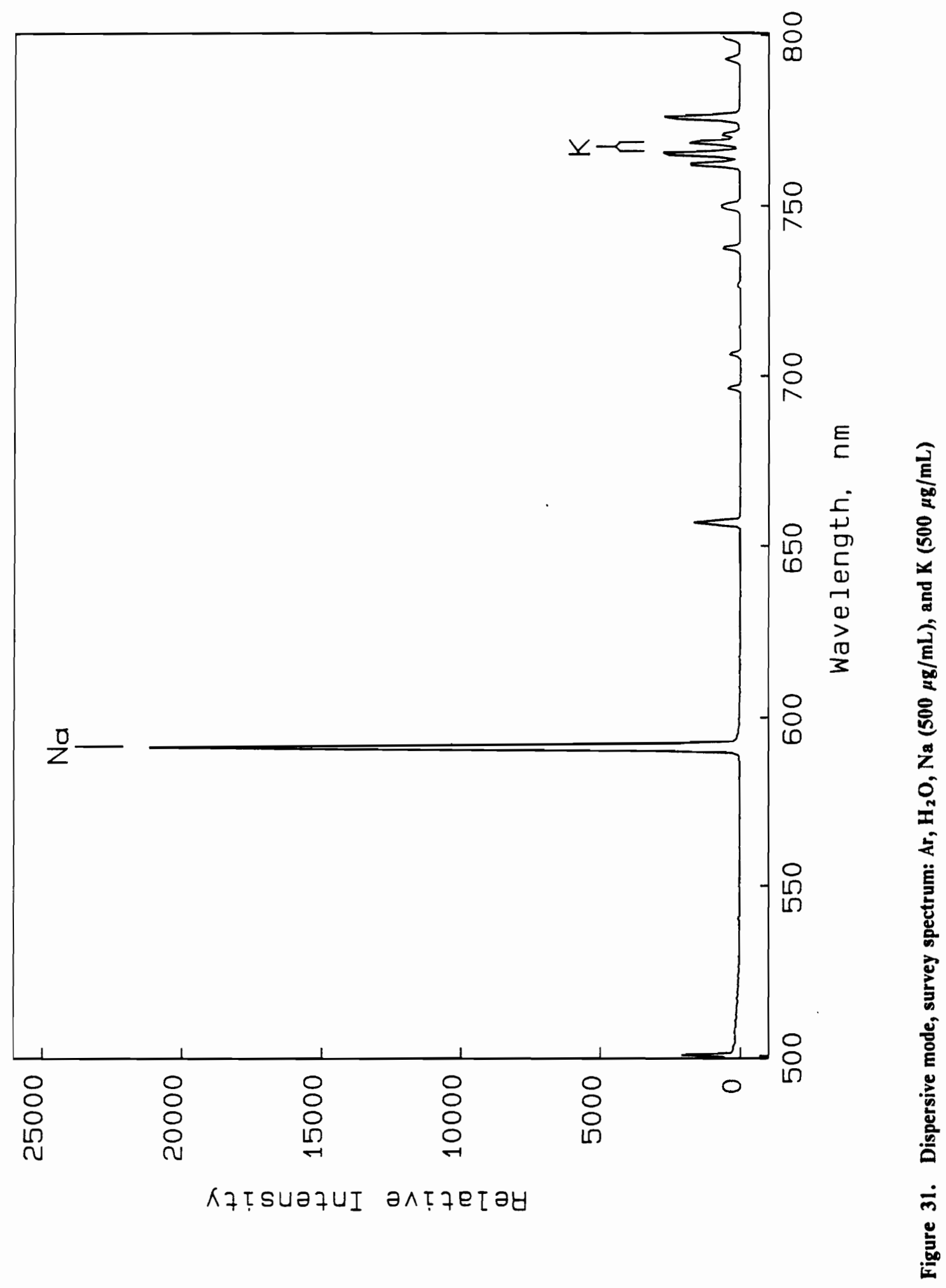




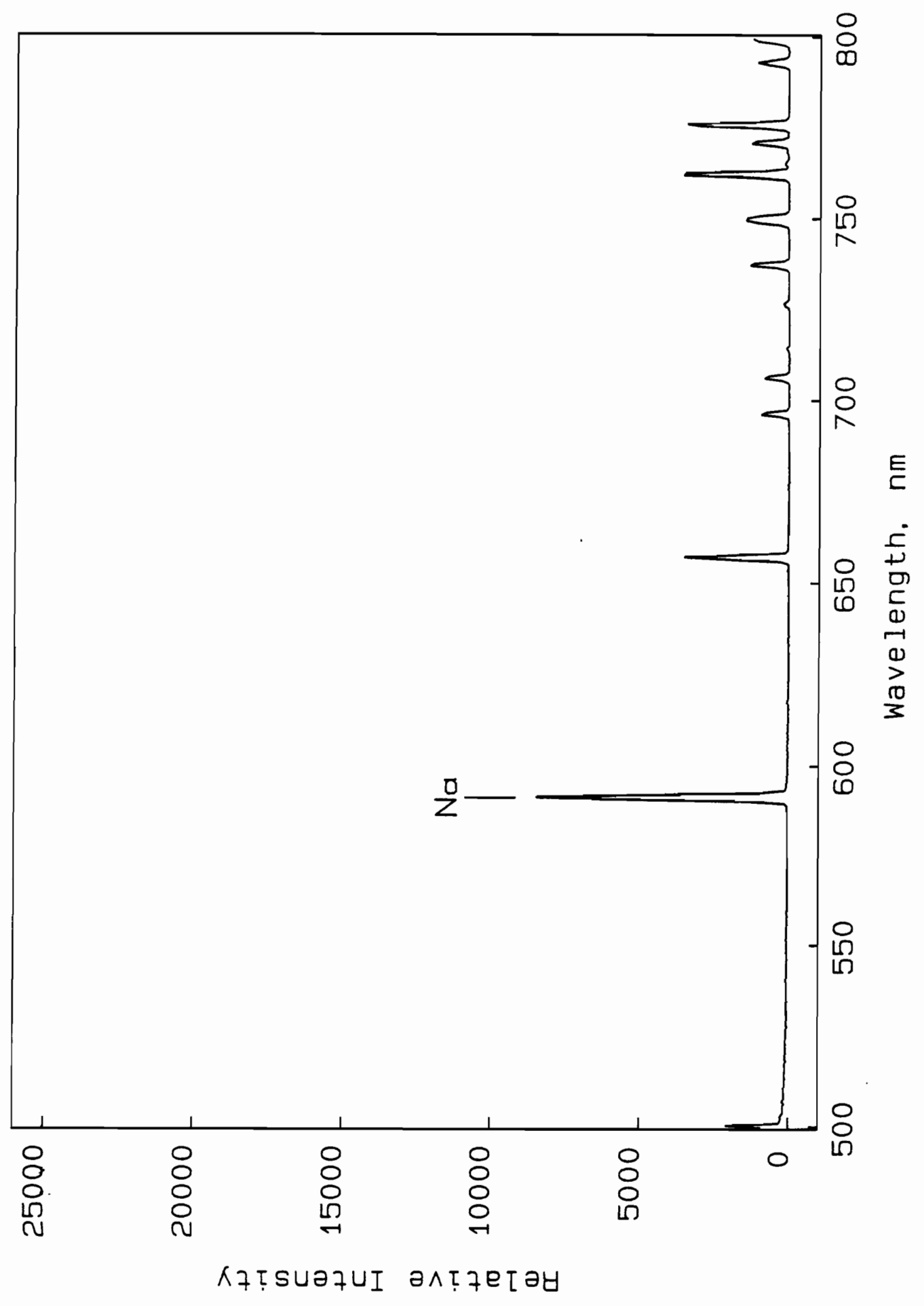

焉 
Table 19. Species Introduced into the MIP for the Survey Spectra

\begin{tabular}{cl} 
Figure & Species \\
\hline 27 & $\mathrm{Ar}$, "dry" frit. \\
28 & $\mathrm{Ar}, \mathrm{H}_{2} \mathrm{O}$. \\
29 & $\mathrm{Ar}, \mathrm{H} 2 \mathrm{O}(500 \mu \mathrm{g} / \mathrm{mL})$ \\
30 & $\mathrm{Ar}, \mathrm{H}_{2} \mathrm{O}, \mathrm{K}(500 \mu \mathrm{g} / \mathrm{mL})$ \\
31 & $\mathrm{Ar}, \mathrm{H}_{2} \mathrm{O}, \mathrm{Na}(500 \mu \mathrm{g} / \mathrm{mL}), \mathrm{K}(500 \mu \mathrm{g} / \mathrm{mL})$ \\
32 & $\mathrm{Ar}, \mathrm{H}_{2} \mathrm{O}, \mathrm{Na}(100 \mu \mathrm{g} / \mathrm{mL})$
\end{tabular}


Table 20. Emission Species in the Survey Difference Spectrum

\begin{tabular}{cclc} 
Figure & $\begin{array}{c}\text { Differences, from } \\
\text { Figures 27 to 32 }\end{array}$ & $\begin{array}{c}\text { Emitting } \\
\text { Species }\end{array}$ & $\begin{array}{c}\text { Analytes and } \\
\text { Concentrations }\end{array}$ \\
\hline 33 & {$[28-27]$} & $\mathrm{H}$ and $\mathrm{O}$ & \\
34 & {$[29-28]$} & $\mathrm{Na}$ & $500 \mu \mathrm{g} / \mathrm{mL}$ \\
35 & {$[30-28]$} & $\mathrm{K}$ & $500 \mu \mathrm{g} / \mathrm{mL}$ \\
36 & {$[31-28]$} & $\mathrm{Na}$ & $500 \mu \mathrm{g} / \mathrm{mL}$ \\
37 & {$[32-28]$} & $\mathrm{K}$ & $500 \mu \mathrm{g} / \mathrm{mL}$ \\
& & $\mathrm{Na}$ & $100 \mu \mathrm{g} / \mathrm{mL}$
\end{tabular}


The detected line intensities in these spectra displayed several deviations from the ideal linear relationship between concentration and intensity. The Na peak heights in Figure 34 due to $500 \mu \mathrm{g} / \mathrm{mL} \mathrm{Na}$, and in Figure 36 due to $500 \mu \mathrm{g} / \mathrm{mL} \mathrm{Na}$ plus $500 \mu \mathrm{g} / \mathrm{mL} \mathrm{K}$, differ by approximate $10 \%$. This could be due to source fluctuation, specifically sample transport from the nebulizer. It could also be due to suppressed $\mathrm{Na}$ emission caused by plasma loading by the $500 \mu \mathrm{g} / \mathrm{mL} \mathrm{K}$. A fair amount of plasma loading by $\mathrm{K}$ is indicated by the $\mathrm{H}$ line in the $\mathrm{K}$ difference spectrum, Figure 35. Another discrepancy is seen in the Na peak heights in Figure 34 and 37. The peak heights, due to $500 \mu \mathrm{g} / \mathrm{mL}$ and $100 \mu \mathrm{g} / \mathrm{mL} \mathrm{Na}$ respectively, do not correspond to the $5: 1$ concentration ratio. These intensity ratios were repeatable under direct observation. The intensity discrepancy could be due to self absorption or plasma loading. Since the major thrust of this research was toward spectrometer design, and not source design, and the specific goal of these experiments was to obtain a qualitative overview of the plasma, these discrepancies were not pursued.

The negative peaks in all of the spectra in Figures 33 to 37 tend to indicate that plasma loading was not a severe problem, except for Figure 36. For this Figure, the total analyte concentration was $1000 \mu \mathrm{g} / \mathrm{mL}$. Plasma loading was, however, a noticeable effect in all of the spectra. Therefore, with an MIP, quantitation errors due to plasma loading should investigated, particularly when total analyte concentration is high.

\section{Nebulizer Equilibration Time.}

The frit nebulizer was chosen for the MMS because of its reduced nebulizer fluctuations relative to other nebulizers. One disadvantage of the frit nebulizer is its long sample equilibration time and long rinse time. The source of the problem is the physical depth and large surface area of the frit. Because of these factors, it takes a long time for a sample to spread over the frit, to displace the rinse solution from the frit volume, and to attain a steady-state nebulization rate. 


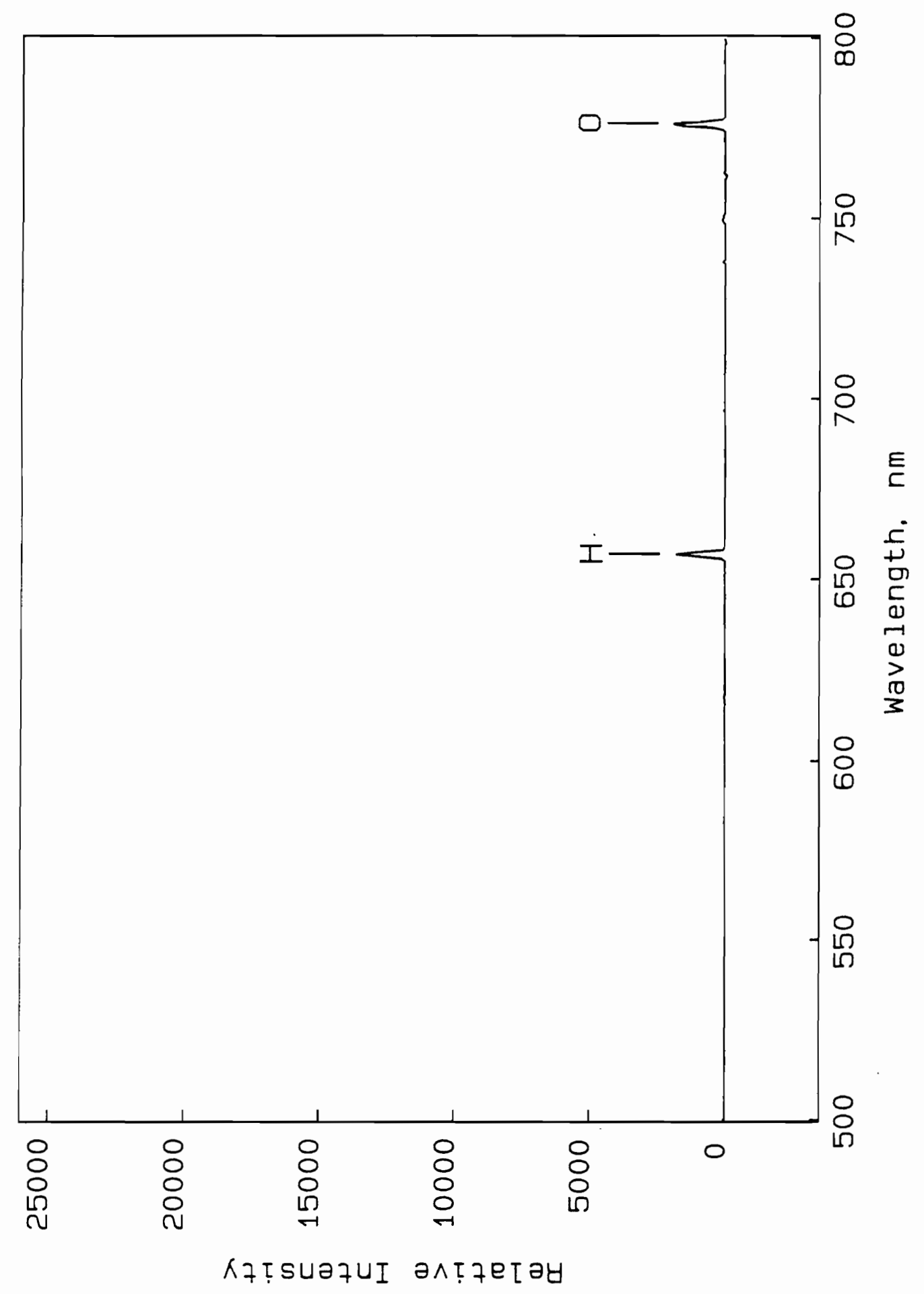

O 


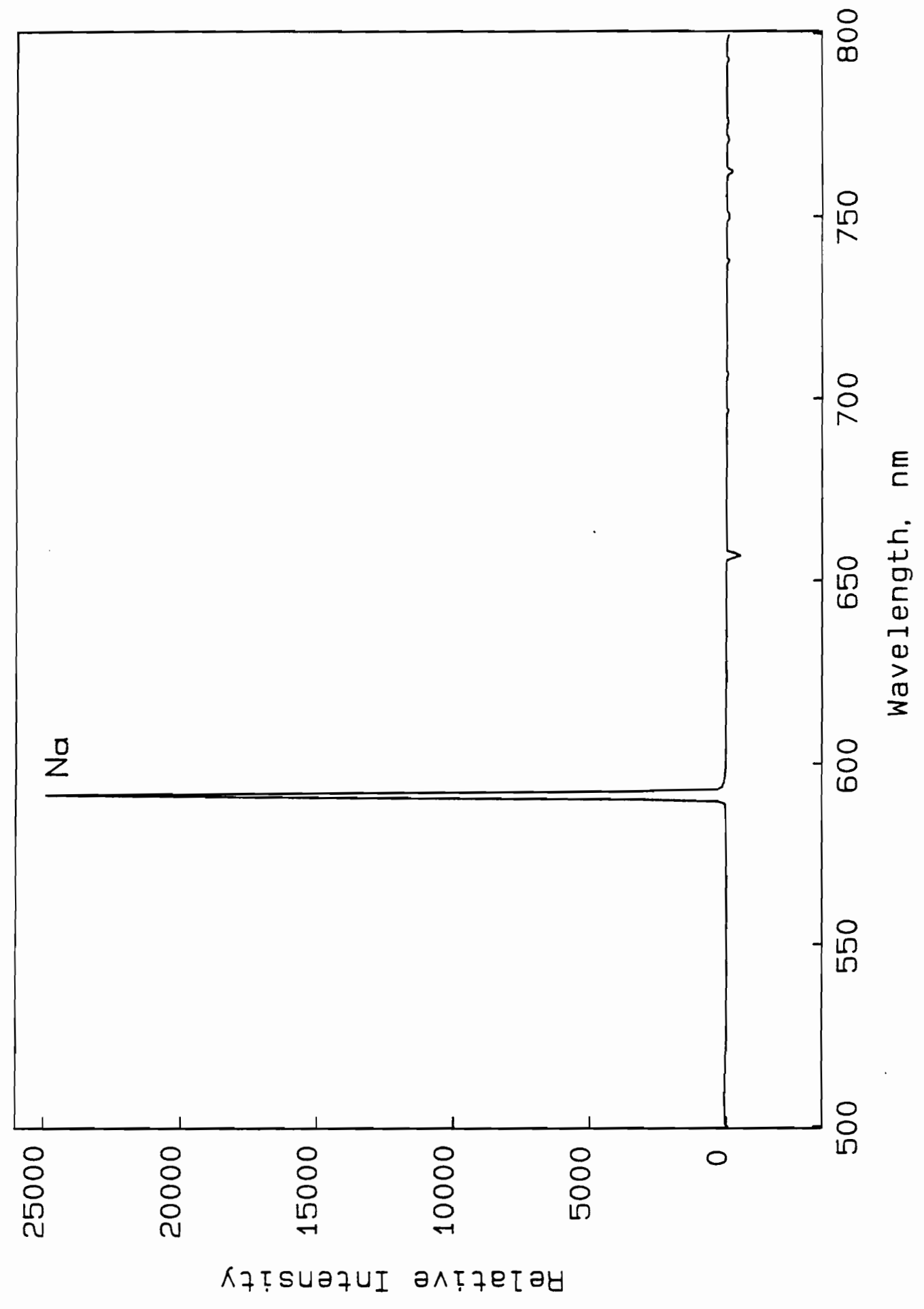

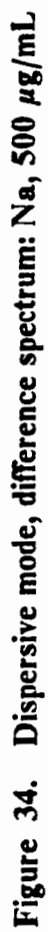




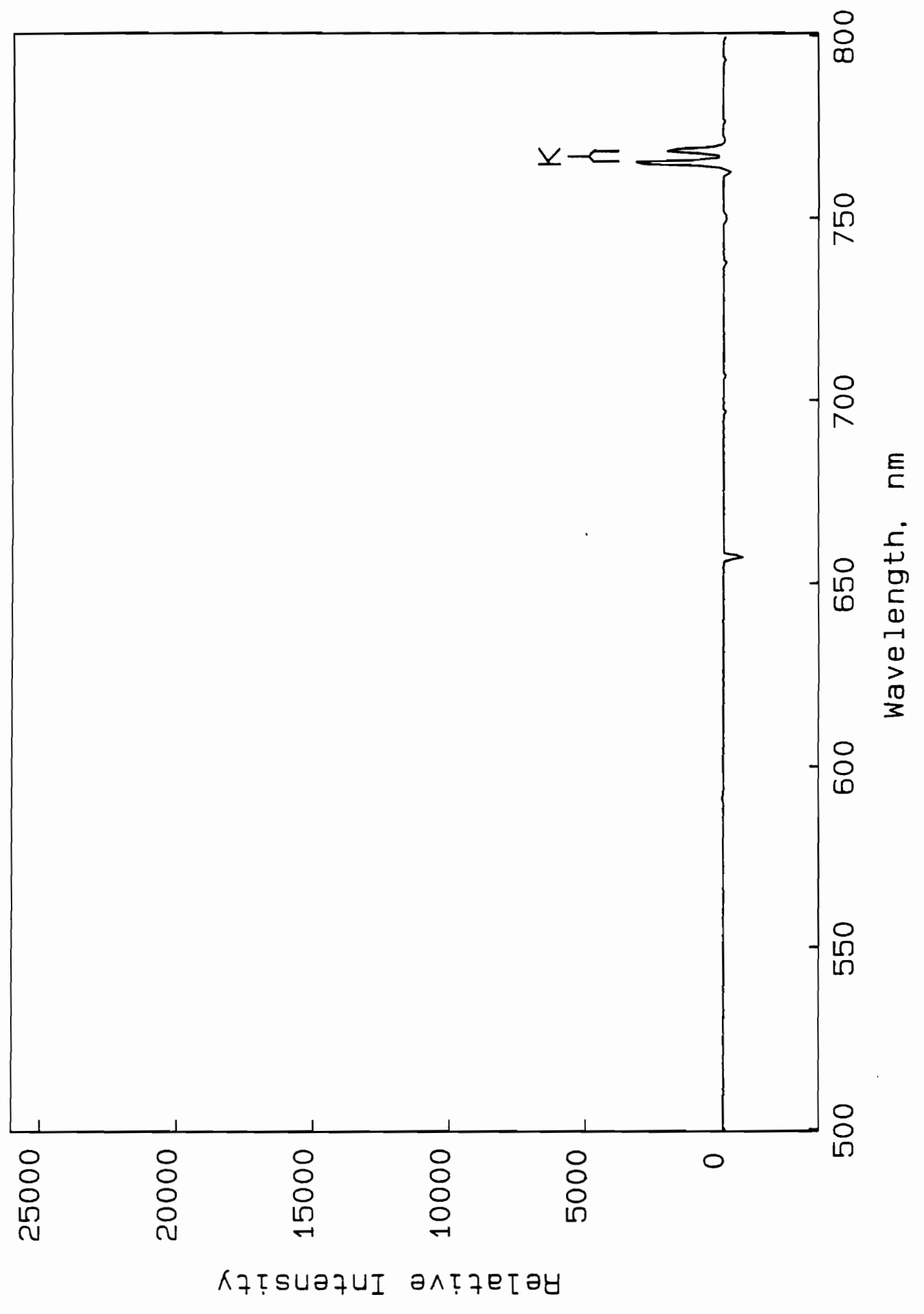

ह 


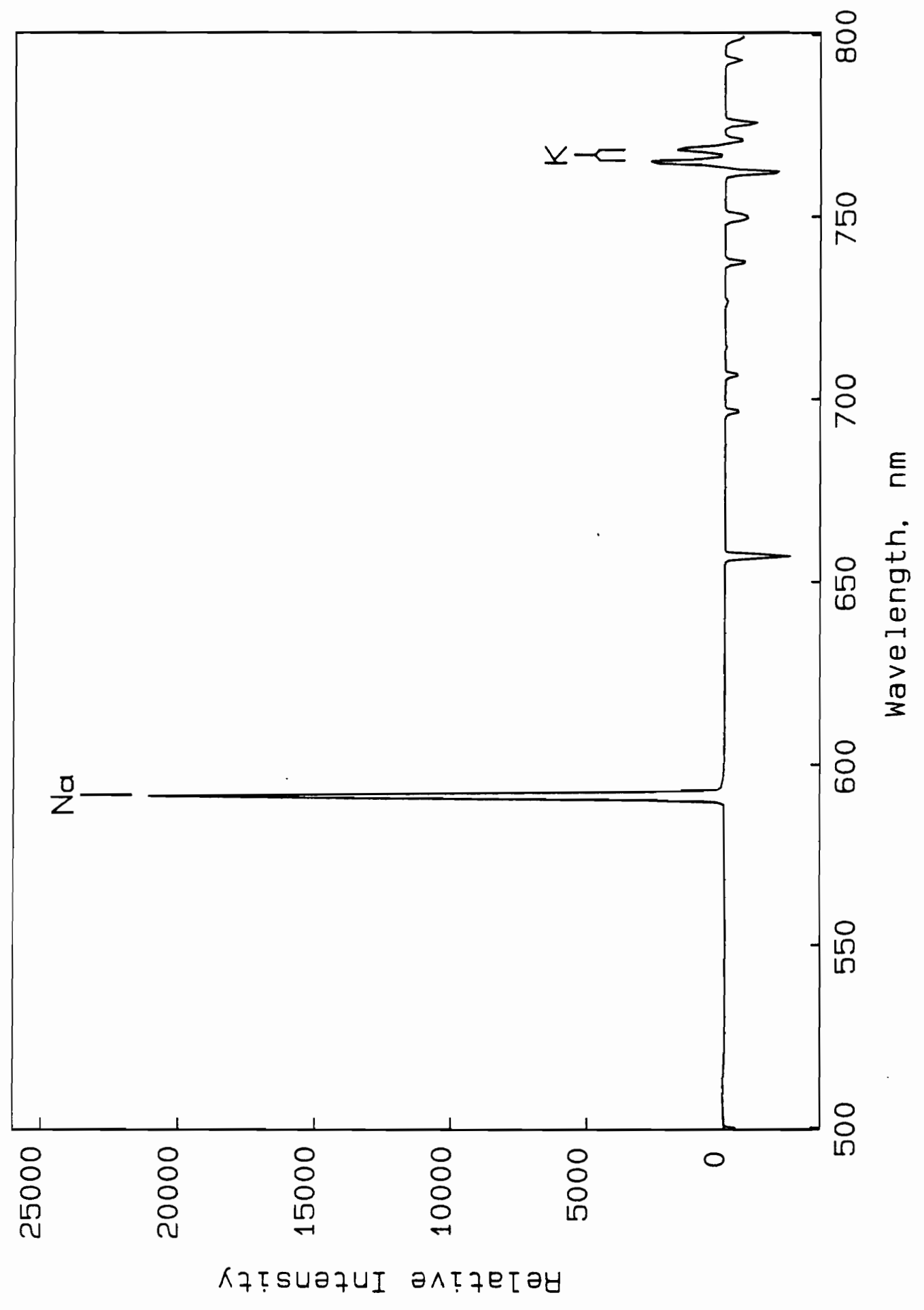

है 


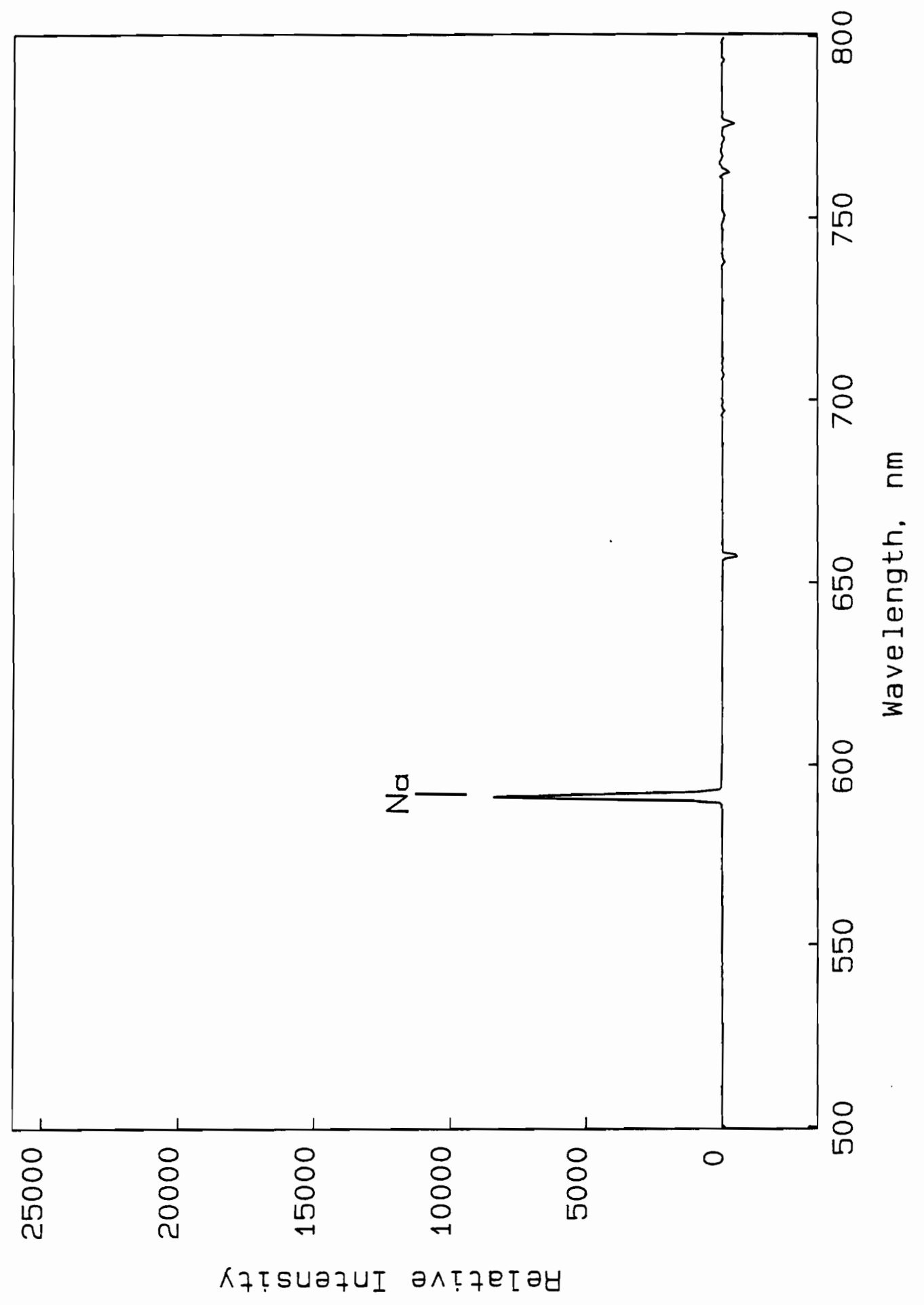

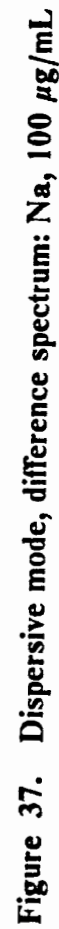


A time-series of spectra was acquired to get a qualitative picture of the nebulizer equilibration time and process, and to examine the intensities of the plasma background lines over the same time frame. Figure 38 shows the spectra as a three-dimensional plot. For these spectra, the MIP was set up in the side-on configuration. To emphasize intensity variations over time, spectra were not coadded. The experimental parameters for the spectra are shown in Table 21. In preparation for data acquisition, a stream of deionized water was pumped onto the frit. Just before data acquisition, a solution of $100 \mu \mathrm{g} / \mathrm{mL}$ of $\mathrm{Na}$ was started on to the frit. Time between spectra was $6 \mathrm{sec}$, and the total elapsed time was $90 \mathrm{sec}$.

The spectra in Figure 38 show a linear increase in the $\mathrm{Na}$ emission over the first nine spectra $(48 \mathrm{sec})$. Extrapolating backward in time, zero intensity for $\mathrm{Na}$ occurred $30 \mathrm{sec}$ before the first spectrum. The last eight spectra $(42 \mathrm{sec})$ indicate that the nebulizer was at or near equilibration. The nebulizer equilibration time was therefore estimated to be approximately $90 \mathrm{sec}$. $\mathrm{Na}$ intensity fluctuations in the last eight spectra were probably due to nebulization rate fluctuations. The Ar background line at $500 \mathrm{~nm}$ indicates the plasma was not perturbed over the sample introduction period. However, the $\mathrm{H}$ line at $656 \mathrm{~nm}$ displayed a linear decrease over this time frame.

\section{End-on Emission Studies.}

End-on emission studies were motivated by concerns of plasma structure, and the possibility of enhanced signal due to a larger sampled plasma volume. Enhanced signal in the end-on geometry is possible because the path length through the plasma is approximately $19 \mathrm{~mm}$ versus $6 \mathrm{~mm}$ for the side-on geometry.

There are a number of visible emission zones within the MIP. The zones observed while nebulizing a Na sample into the MIP are shown in Figure 25. In the center of the plasma there is a very thin, intense filament. This filament appears as a very luminous white-purple region. It 


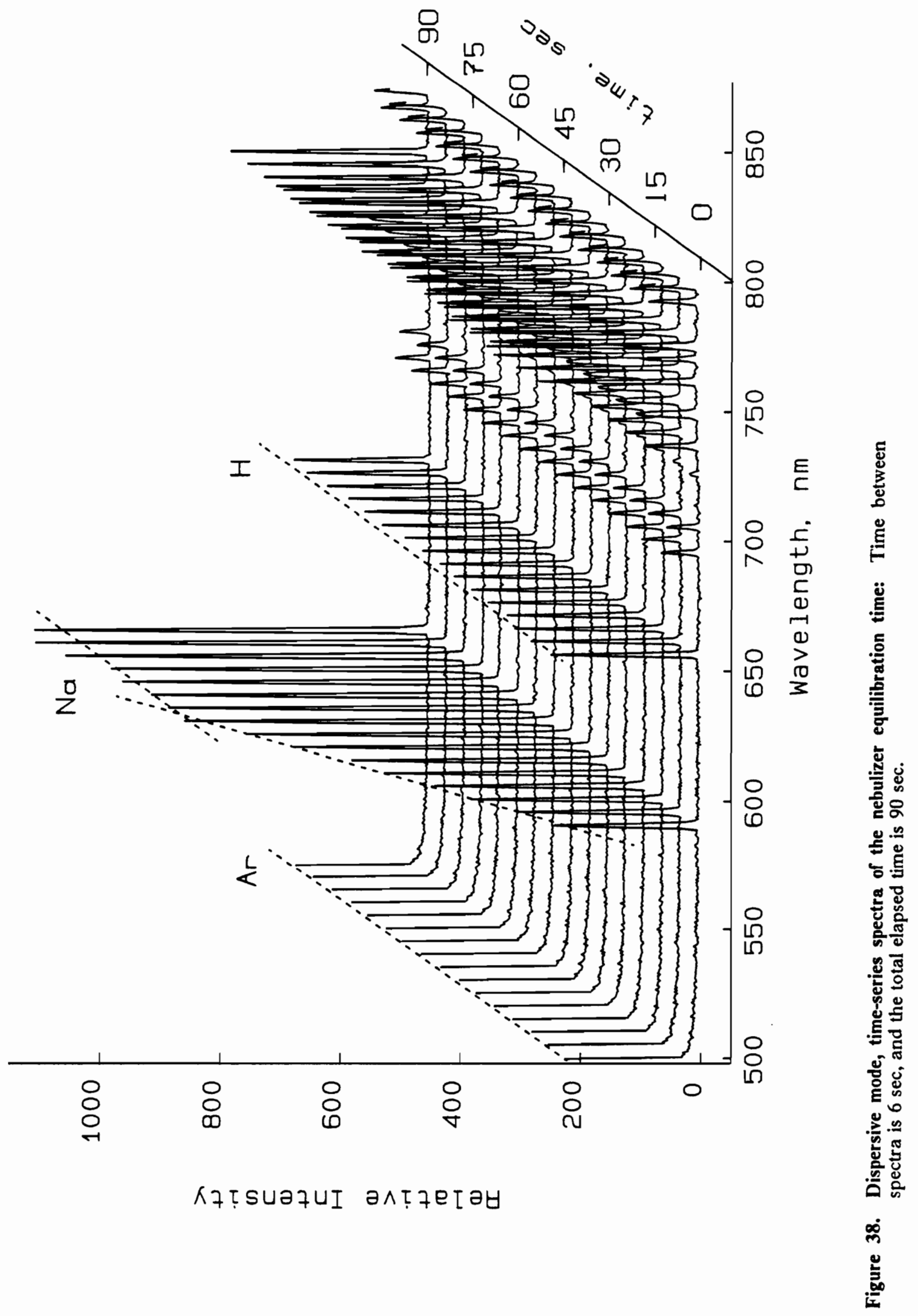


Table 21. Experimental Parameters for the Nebulizer Equilibration Time Spectra

Experimental parameters:

MIP parameters:

Sample:

Viewing geometry:

$\mathrm{Na}, 100 \mu \mathrm{g} / \mathrm{mL}$

Side-on

Observation plane 1, $0.5 \mathrm{~mm}$ from cavity face.

Scans Sequence:

Number of scans:

16

Time between scans:

Total elapsed time:

$6 \mathrm{sec}$

$90 \mathrm{sec}$

MMS operating parameters:

Mode:

Dispersive

Spectral Range:

$500-800 \mathrm{~nm}$.

Spectral bandwidth:

$300 \mathrm{~nm}$

Points per Array Scan:

Transfer aperture:

Resolution:

512

Average resolving power:

$1.000 \times 0.050 \mathrm{~mm}$.

$1.2 \mathrm{~nm}$

540

Integration time:

$33.3 \mathrm{msec}$

Coadded scans per spectrum:

1

Total exposure time

per spectrum:

$33.3 \mathrm{msec}$. 
appears to have considerable spectral background, with possibly intense UV emission. To the eye, it does not display any yellow $\mathrm{Na}$ emission. Immediately around the central filament is a region with essentially the same emission characteristics as the central filament, but with less intensity. These first two regions comprise the Ar emission core. The third region is a large annulus, or cylindrical volume, which displays considerable yellow Na emission. When nebulizing water, this region is transparent, with the emission characteristics of the first two regions, but with considerably less intensity. The last region within the cavity is a boundary layer region next to the quartz tube. This region shows little intensity, due to cooling by the tube. One region of interest outside of the cavity is the conical plume extending from the end of the plasma. This region should be populated by cold, absorbing sample atoms. This region is much thicker for the end-on observation than for side-on observation, and thus a source of potential problems with self-absorption and self-reversal.

In preparation for end-on spectra, the optics of the MMS were modified by placing a quartz optical widow, OW in Figure 26, in front of the collimating lens. The window protected the lens from heating and sample deposition by the plasma. The MIP was then placed in the focal plane of the MMS, and a $100 \mu \mathrm{g} / \mathrm{mL}$ Na sample was pumped into the nebulizer. The MIP was manipulated in the focal plane until a clear image of the end-on emission zones was projected onto the center of the transfer aperture, A1. The MIP was actually positioned so that the focal plane was several millimeters inside of the cavity. This allowed a greater plasma volume to be sampled by the optical sampling cones of the MMS. Final MIP positioning was done by monitoring the detected emission intensity of the plasma with the MMS, which was in free-running dispersive mode operation.

Figure 39 shows the spectrum from the Ar emission core, obtained from observation plane 2 in Figure 25. Figure 40 shows the spectrum from the $\mathrm{Na}$ emission annulus, obtained from observation plane 3 in Figure 25. The experimental parameters for these spectra are listed in Table 22, and are the same as those for Figures 27 to 32 , the side-on survey spectra. The sample concentration, $100 \mu \mathrm{g} / \mathrm{mL}$, is the same as that for Figure 32. Figure 32 and Figure 28, the side-on DI water background spectrum, are shown rescaled in Figures 41 and 42 for comparison. 


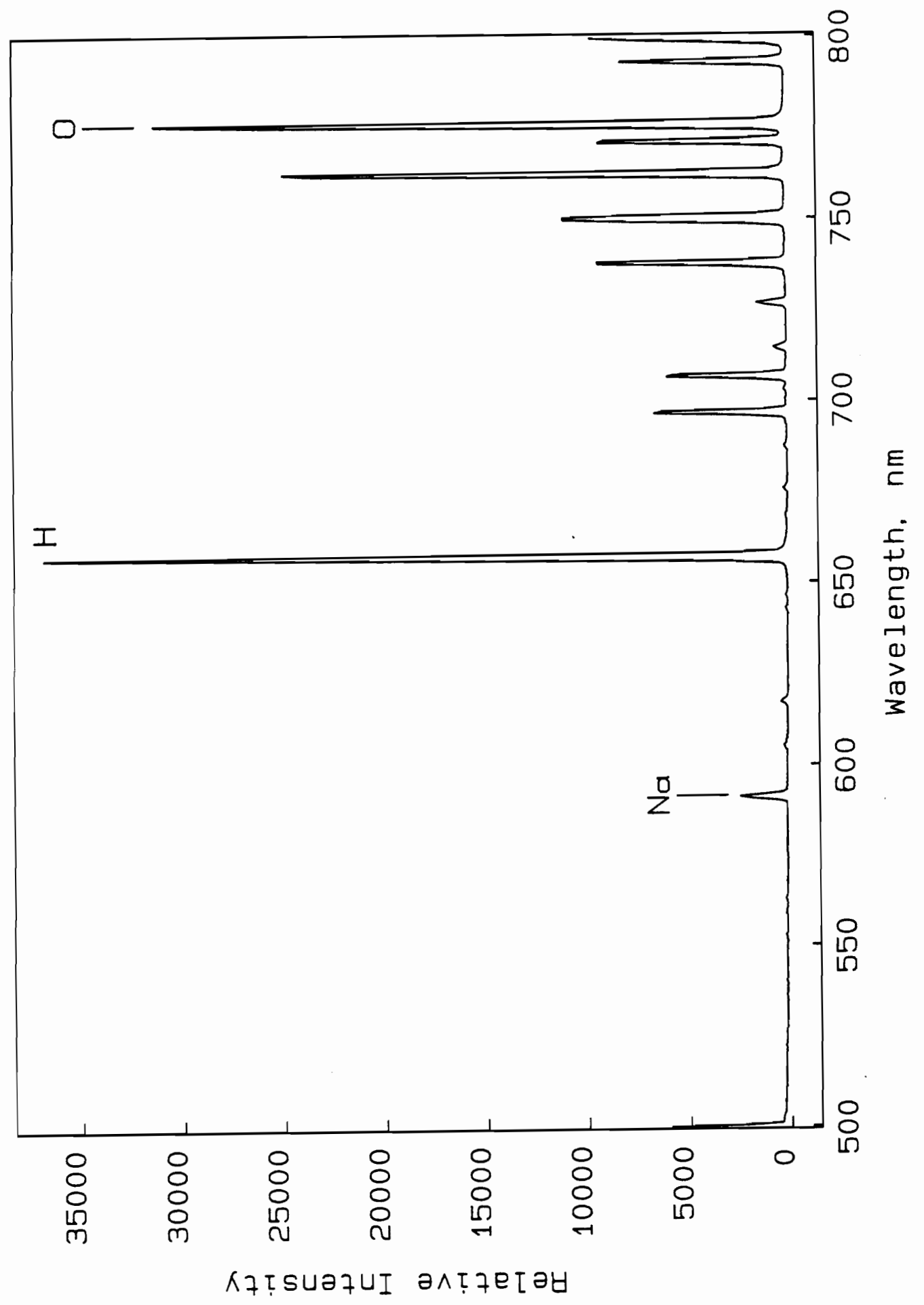




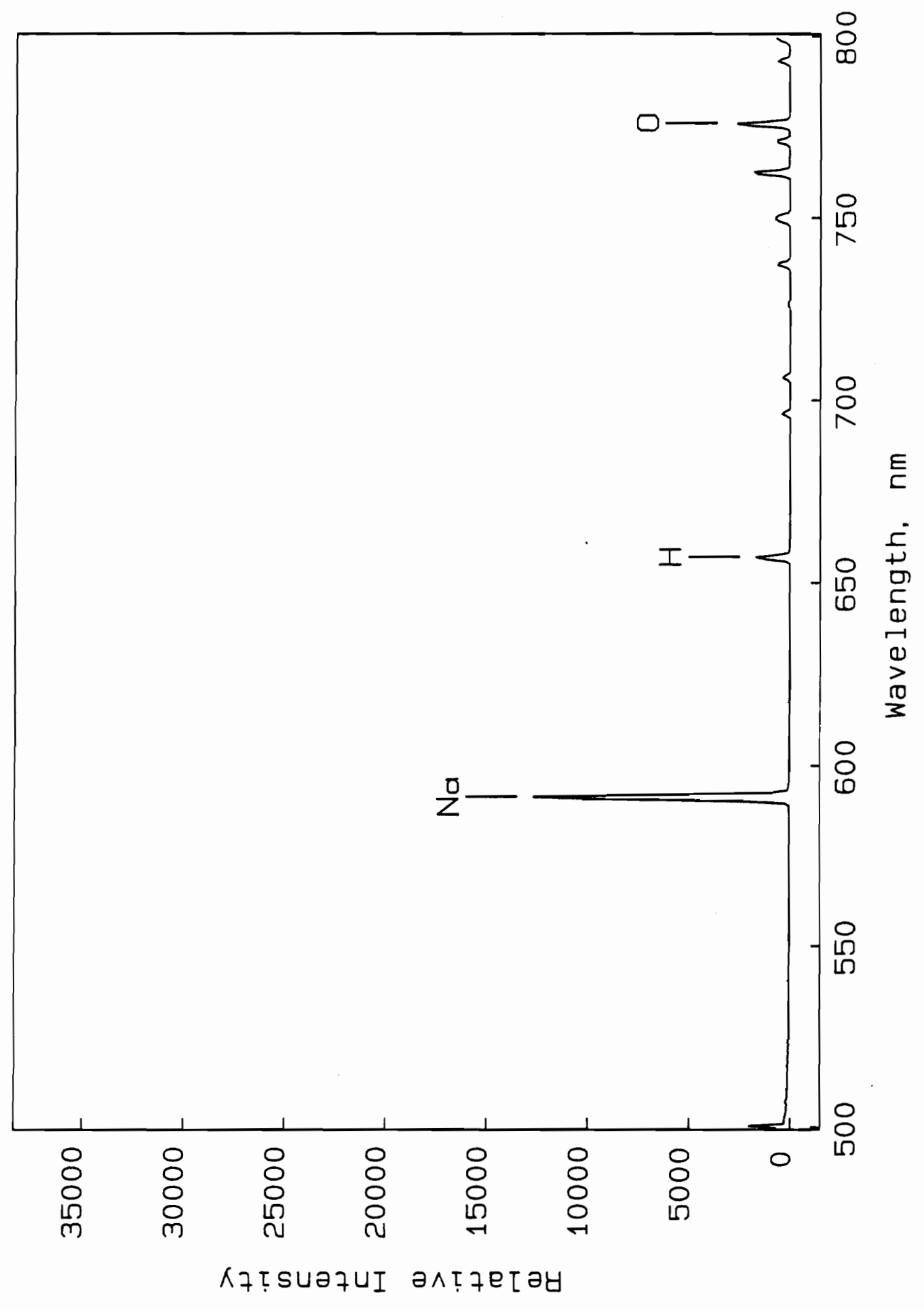

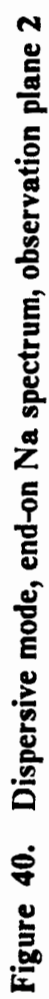




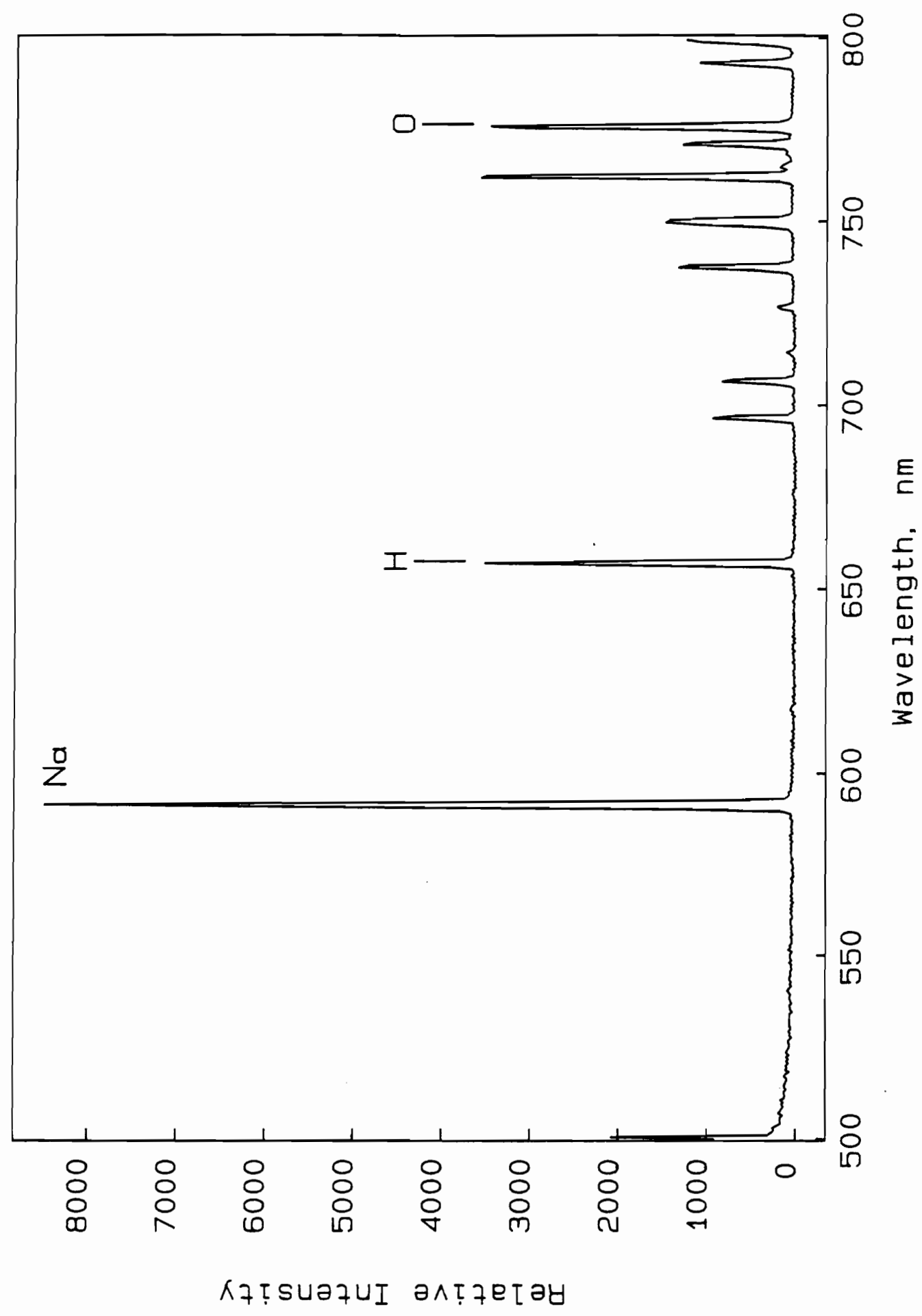

 


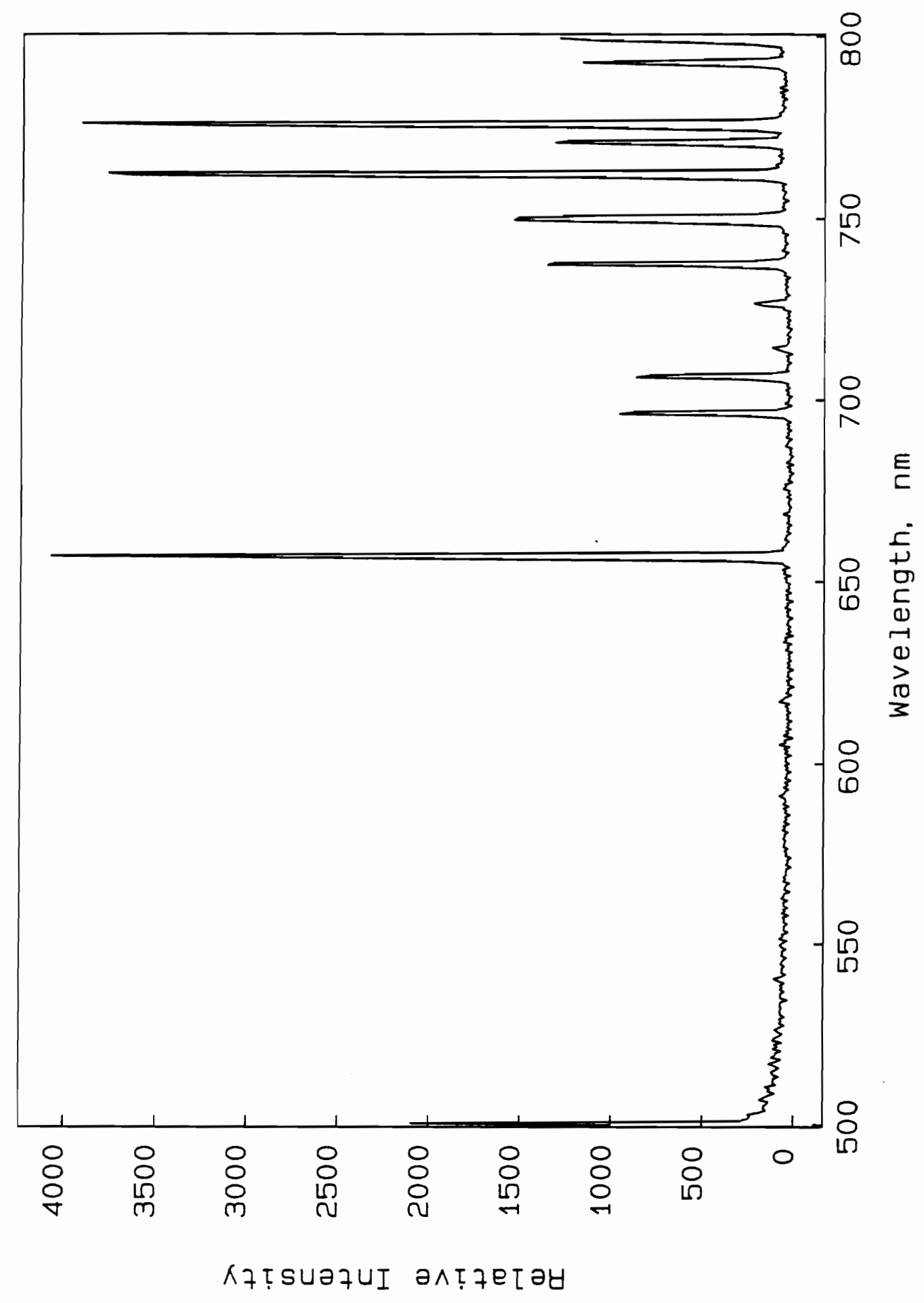

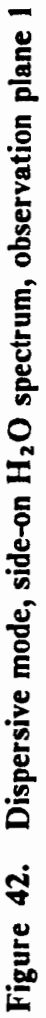


Table 22. Experimental Parameters for the End-on Emission Zone Spectra

Experimental parameters:

MIP parameters:

Sample:

Viewing geometry:

Figure 39, Ar zone

Figure $40, \mathrm{Na}$ annulus

MMS operating parameters:

Mode:

Spectral Range:

Spectral bandwidth:

Points per Array Scan:

Transfer aperture:

Resolution:

Average resolving power:

$\mathrm{Na}, 100 \mu \mathrm{g} / \mathrm{mL}$

Side-on

Observation plane 1,

$0.5 \mathrm{~mm}$ from the plasma center Observation plane 2 ,

$2.0 \mathrm{~mm}$ from the plasma center

Integration time:

Coadded scans per spectrum:

Total exposure time

per spectrum:

Dispersive

500-800 nm.

$300 \mathrm{~nm}$

512

$1.000 \times 0.050 \mathrm{~mm}$.

$1.2 \mathrm{~nm}$

540

$33.3 \mathrm{msec}$

10

333 msec. 
As the MIP was translated across the focal plane of the MMS, considerable differences in spectra as a function of radial position was observed. The spectra in Figures 39 and 40 reflect these differences. Near the center of the plasma the Ar lines became very strong, saturating the detector at the plasma center, while the $\mathrm{Na}$ emissions were greatly reduced. Figure 39 shows the spectrum obtained $0.5 \mathrm{~mm}$ from the center of the plasma, on observation plane 2 in Figure 25, just off of the spatial Ar emission maximum. This position was chosen to prevent detector saturation.

The emission trends reversed when observing in the $\mathrm{Na}$ emission annulus. The Ar lines were greatly reduced, and the $\mathrm{Na}$ line became much stronger. Figure 40 shows the spectrum obtained from the $\mathrm{Na}$ annulus, $2.0 \mathrm{~mm}$ from the center of the plasma, observation plane 3 in Figure 25. The maximum $\mathrm{Na}$ intensity versus radial displacement was displayed at this position.

The line intensities and the intensities ratios between the spectra in Figures 39 to 42 are summarized in Tables $23 \mathrm{~A}$ and $23 \mathrm{~B}$ respectively. Consider first the emission intensities from the Ar core versus the other two observation geometries. Table 23B shows that the $\mathrm{Na}$ intensity in the Ar core is only about $20 \%$ to $30 \%$ of the $\mathrm{Na}$ intensity in the other two geometries. The background line intensities, however, are 11 to 22 time greater in the Ar core. Consider next the intensity ratios for the $\mathrm{Na}$ emission annulus, the end-on geometry. Table $23 \mathrm{~B}$ shows that the $\mathrm{Na}$ intensity in the annulus is between 1.5 and 5 times greater than in the other two geometries. The intensity of the background lines is lower in the $\mathrm{Na}$ annulus, between $5 \%$ and $75 \%$ of the background intensities in the other 2 geometries.

The intensity differences are probably due to two factors. First, the $\mathrm{Na}$ atoms were probably converted into $\mathrm{Na}$ ions in the Ar core. The Ar core is a very energetic environment, which is indicated by the greatly increased background intensities; compare Figure 39 to 42 . $\mathrm{Na}$, an easily ionizable element, can be expected to exist as an ion rather than an atom in this high energy environment. Useful $\mathrm{Na}$ ion lines do not occur in the spectral region monitored by the MMS, 500-800 nm, but occur at wavelengths of $412 \mathrm{~nm}$ and shorter. The increased $\mathrm{Na}$ intensity in the cooler $\mathrm{Na}$ annulus probably reflects a shift in the $\mathrm{Na}$ ion/ $\mathrm{Na}$ atom equilibrium, producing a larger population of $\mathrm{Na}$ atoms. Because $\mathrm{Na}$ is easily ionizable, the spatial emission trend displayed by $\mathrm{Na}$ may not be a good predictor for the spatial emission trends of other elements. Other elements, 
Table 23. End-on Emission Summary: Ar Core vs. Na Annulus

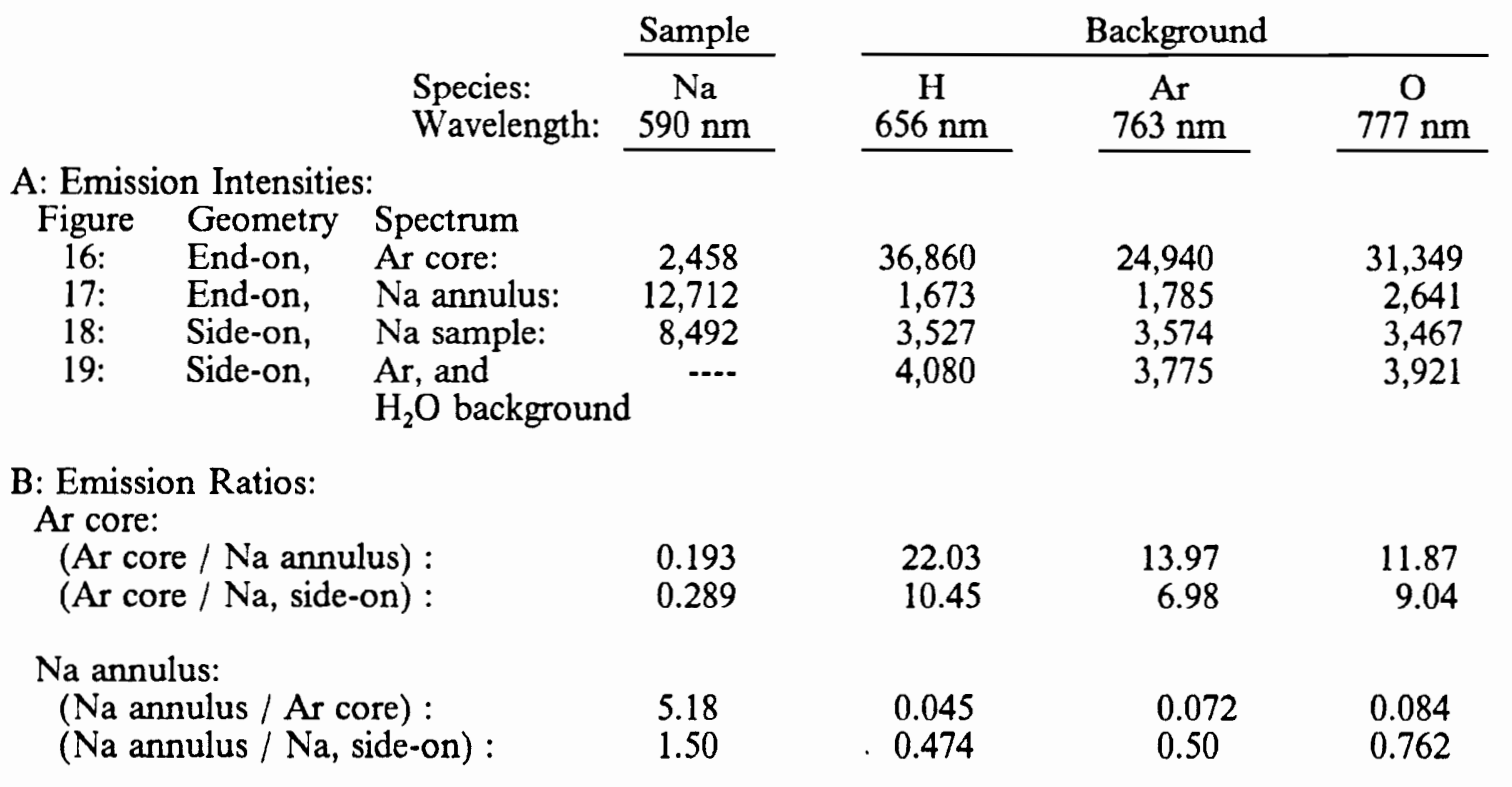


particularly in refractory compounds, may show intensity maxima within the energetic central core region.

The second factor contributing to the emission trends as a function of observation geometry is the averaging effect of the side-on geometry. As can be seen in Figure 25, the side-on geometry samples portions of the $\mathrm{Ar}$ core and the $\mathrm{Na}$ annulus. Because of this, the side- on geometry should show intermediate intensities relative to the two end-on geometries. This fact is born out by the intensity data in Table $23 \mathrm{~A}$.

\section{Interferometric Mode: End-on versus Side-on Observation:}

Interferometric mode studies of the MIP were conducted to determine the MIP signal and noise in this operating mode for side-on and end- on observation geometries. From a strict measurement perspective, the purpose of these studies was to find the observation geometry with the maximum signal, the minimum noise, but ultimately the maximum signal-to-noise ratio $(\mathrm{S} / \mathrm{N})$.

From a diagnostic perspective, there was specific interest in frequencies produced by physical processes in the plasma. Previous studies have reported plasma frequency effects in ICPs 54,49 and MIPs, ${ }^{96,97}$ with some effects directly attributable to plasma gas swirl or plasma rotation, ${ }^{98}$ pump noise, ${ }^{58}$ and power supply fluctuations. ${ }^{49}$ The purpose of this study was to observe radially through the plasma and also longitudinally into the MIP cavity; and then to use the signal and frequency-spectrum analysis capabilities of the MMS to analyze the signal and noise frequency spectra. Specific information was sought on noise level and structure as a function of observation geometry.

One significant difference between the interferometric and the dispersive mode studies was the size of the transfer aperture. In the interferometric studies a $5.000 \mathrm{~mm} \times 0.250 \mathrm{~mm}$ aperture was used; a $1.000 \times 0.050 \mathrm{~mm}$ aperture was used for the dispersive studies. The aperture areas and their projections into the plasma are shown in Figure 43. The larger aperture was used in the 
interferometric studies because it provided 25 times the optical throughput of the smaller aperture. But in addition, the larger aperture sampled, and averaged, a different volume of the source. This effect is illustrated in Figure 43. The interferometric studies used only observation planes 1 and 3. These choices were based upon the dispersive mode results, above.

The interferometric mode data are shown in Figures 44 to 49 . Note that the vertical offset and scale is different for each Figure, permitting display of maximum detail. The experimental parameters for the data are listed in Table 24. The data was taken at fairly low resolution to rapidly survey the plasma and to provide unaliased noise data to $800 \mathrm{~Hz}$. Figures 44,46 , and 48 show side-on data; Figures 45, 47, and 49 show end-on data. Figures 44 and 45 show the side-on and end-on interferograms. Figures 46 and 47 show the side-on and end- on amplitude spectra for the full sample-limited bandwidths. The noise spectra for the respective data also appear in the baselines of Figures 46 and 47 . Figures 48 and 49 show the amplitude spectra over the $400 \mathrm{~cm}^{-1}$ at the $\mathrm{Na}$ lines. A summary and a comparison of the signal, noise, and $\mathrm{S} / \mathrm{N}$ are listed in Table 25 for the two observation geometries.

The side-on interferogram, Figure 44, shows good modulation. This is reflected in the amplitude of the $\mathrm{Na}$ lines in Figures 46 and 48, the corresponding amplitude spectra. The noise in the side-on amplitude spectrum, Figure 46, appears to be white except, for the single- frequency noise at $120 \mathrm{~Hz}$. No other features appear which would indicate plasma rotation or other regular fluctuation. A noise analysis was performed on the baseline noise by calculating the standard deviation of the noise over a number of ranges in the baseline. The noise analysis confirmed that the noise is white, with a standard deviation of 3.77. The $\mathrm{S} / \mathrm{N}$ for the major $\mathrm{Na}$ line is 1,240 .

The end-on interferogram, Figure 45, is more diffuse and shows less modulation than the side-on interferogram. The lower modulation is reflected in the lower amplitude of the $\mathrm{Na}$ lines in Figures 47 and 49, the end-on amplitude spectra. The end-on noise spectrum, the baseline in Figure 47, appears to be white except for the single-frequency noise at $120 \mathrm{~Hz}$. Again, there is no indication of single- frequency noises. The baseline noise analysis confirmed that the noise is white, with a standard deviation of 5.68 . The $\mathrm{S} / \mathrm{N}$ for the major $\mathrm{Na}$ line is 711 . 


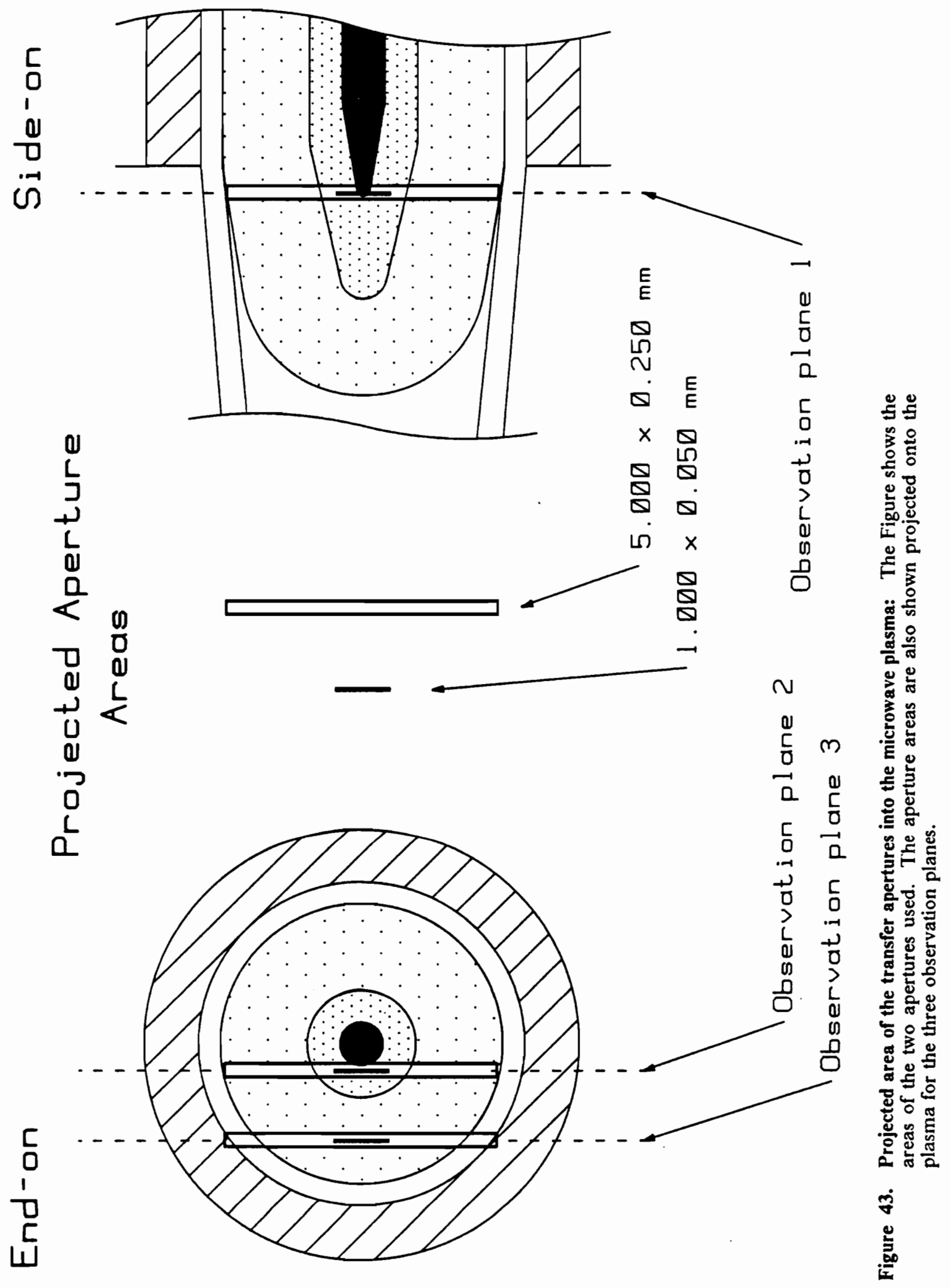




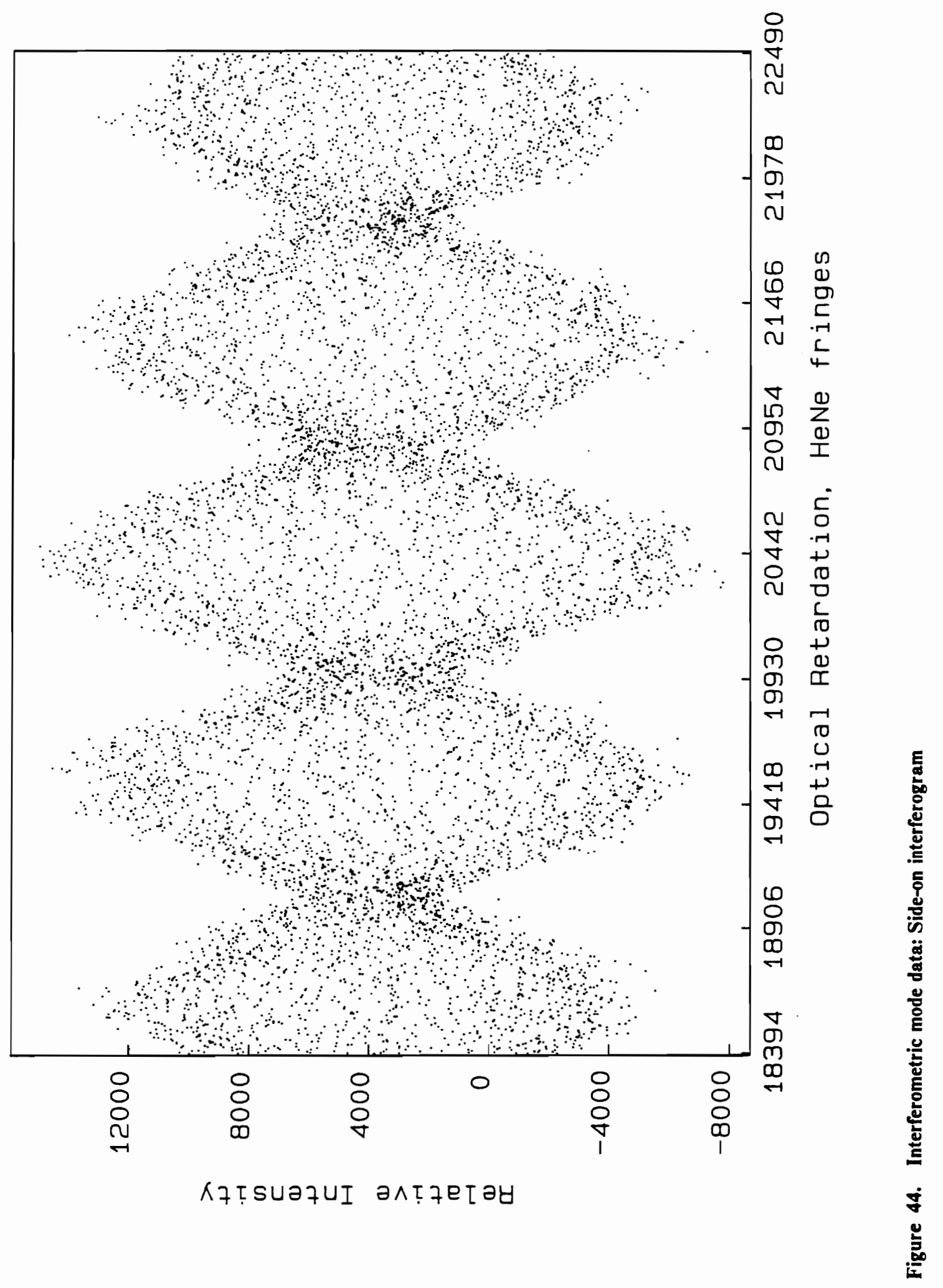




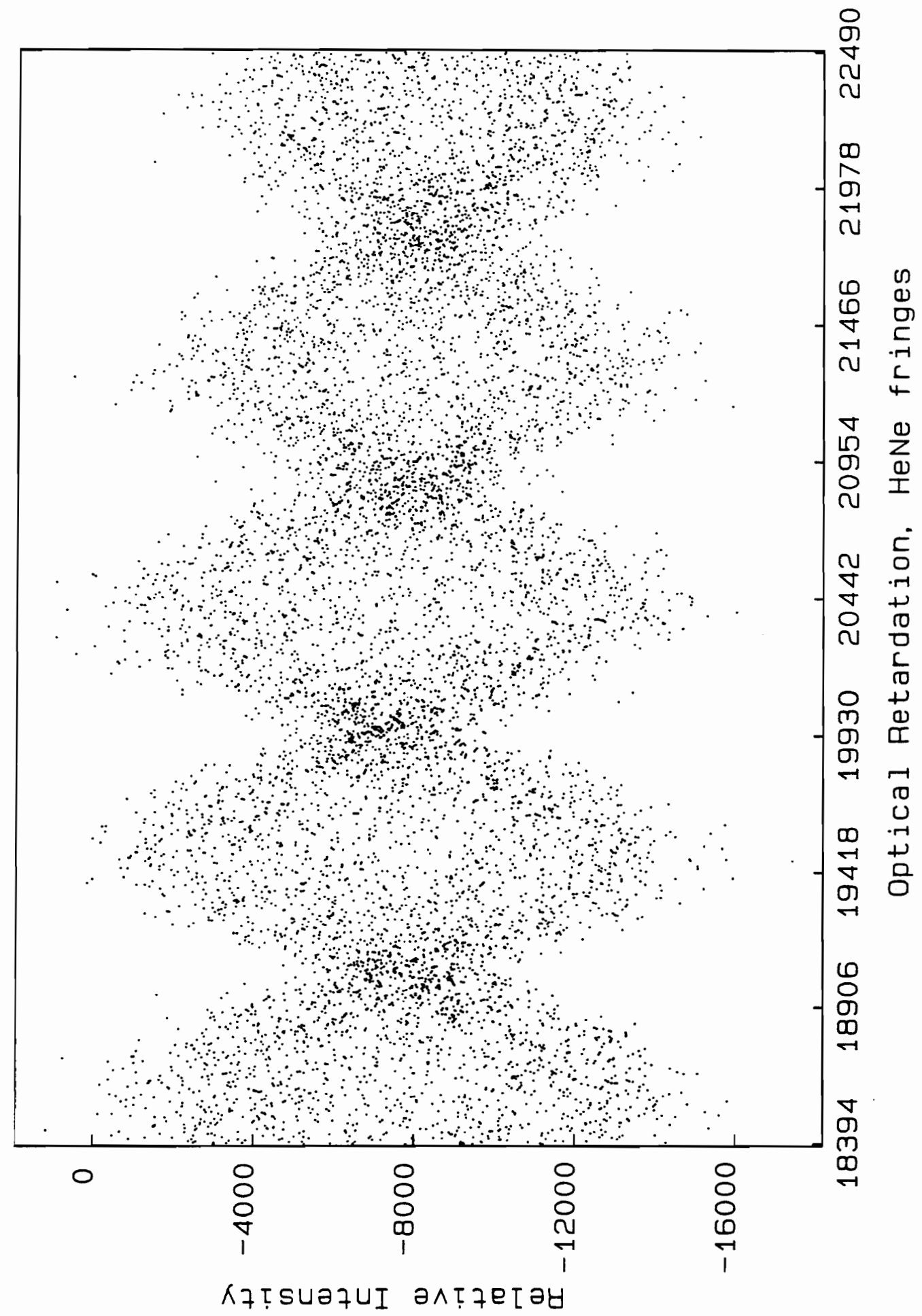

E 


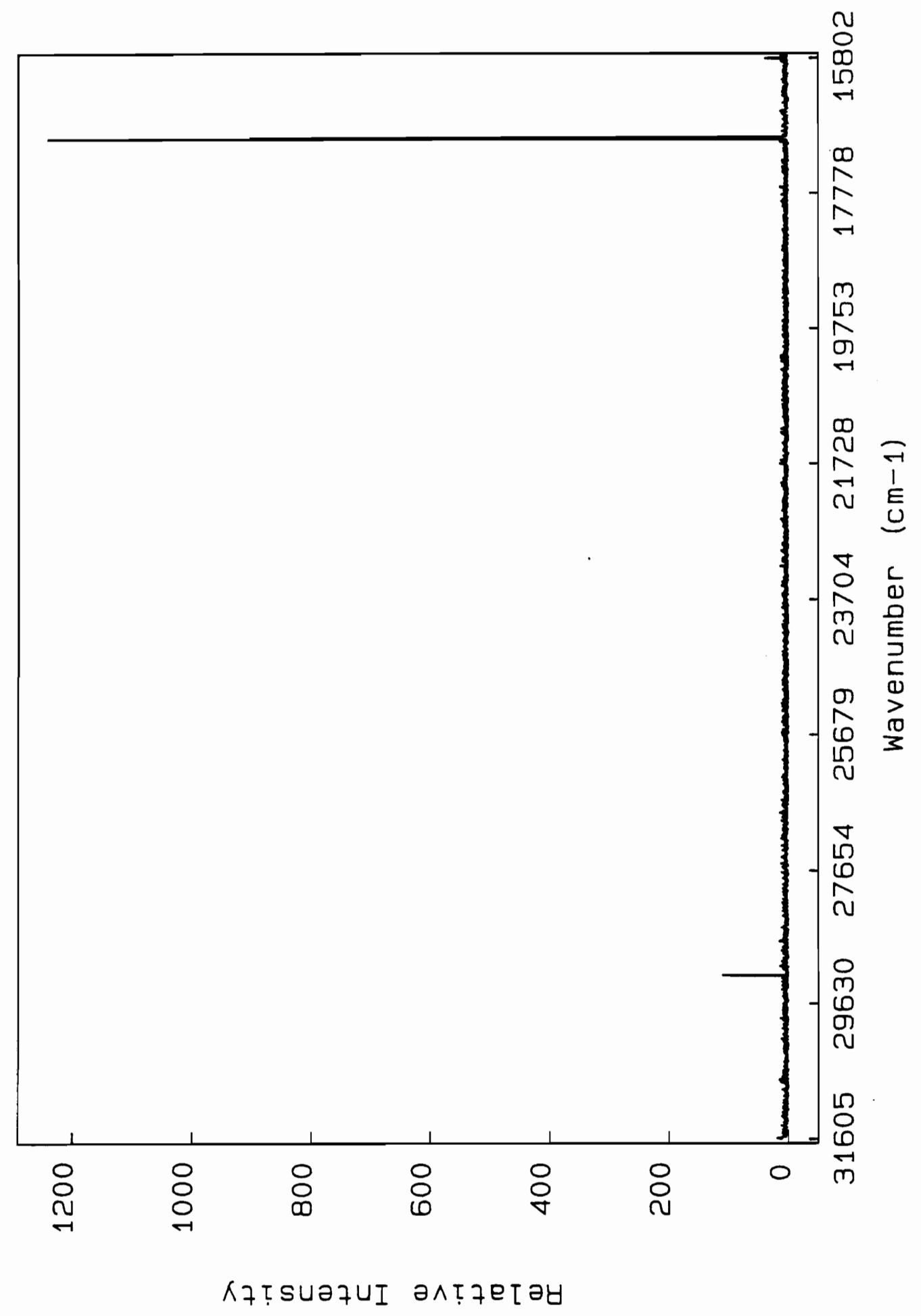

Oे 


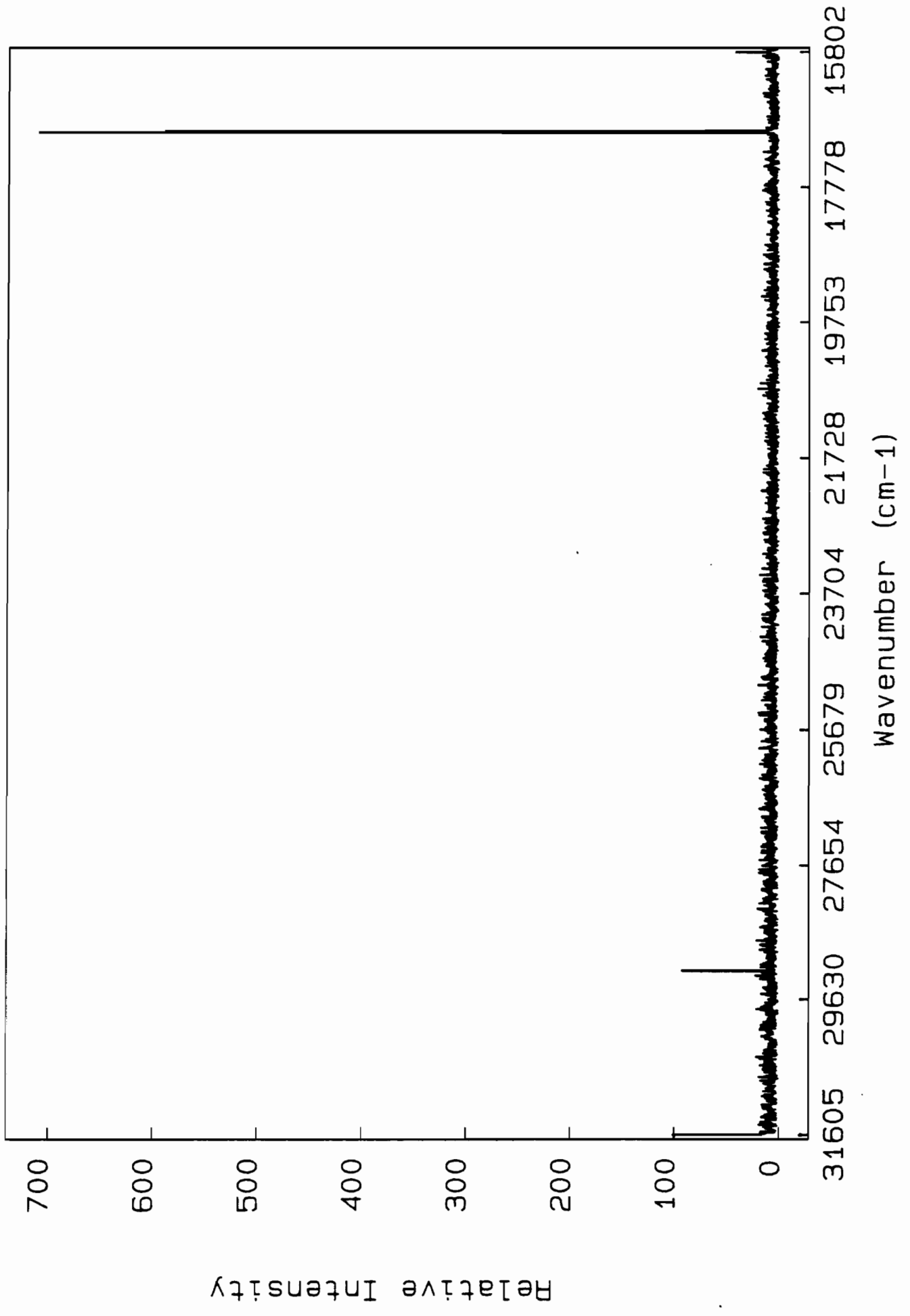

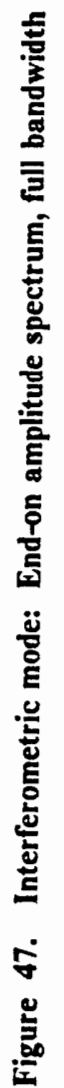




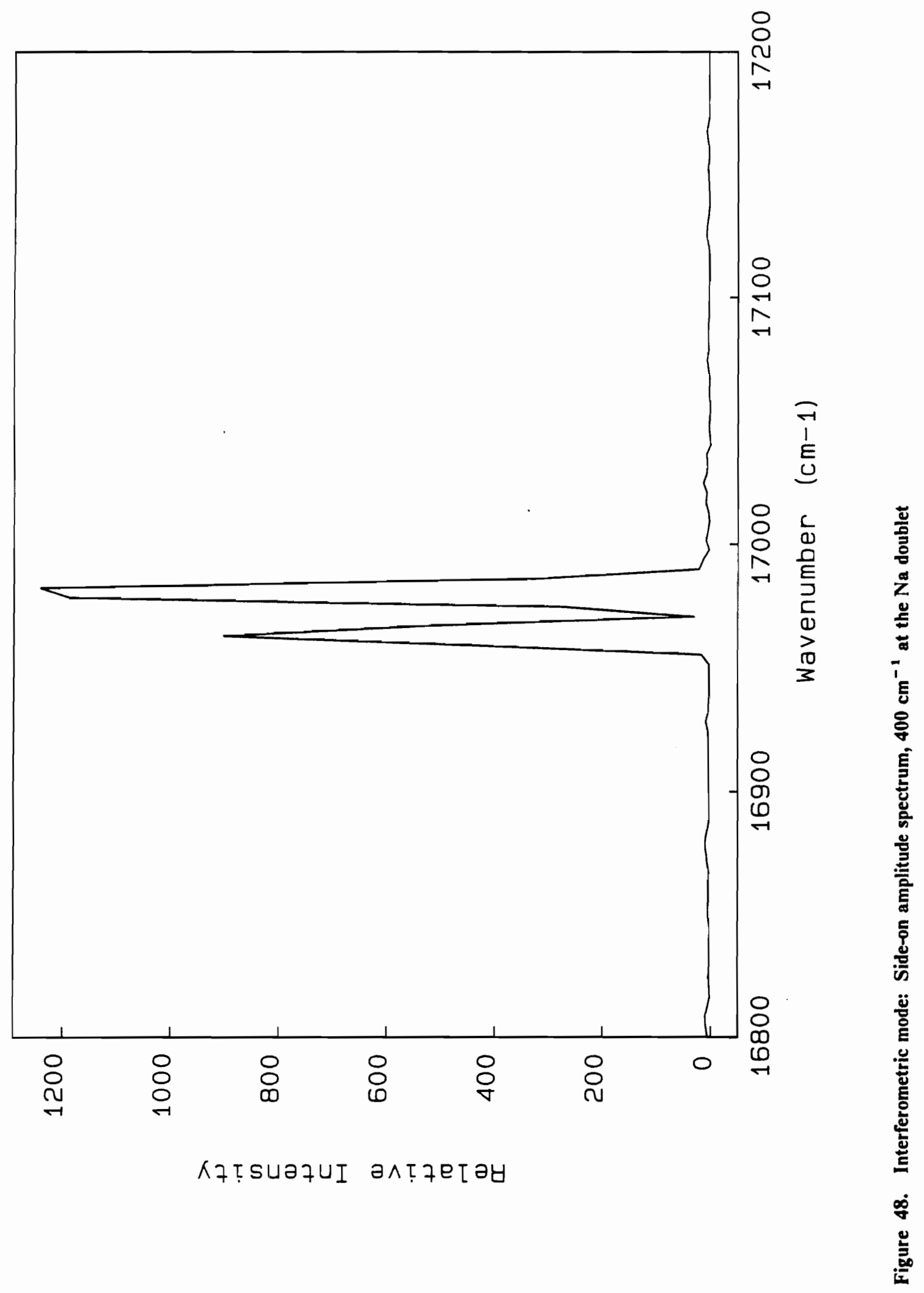




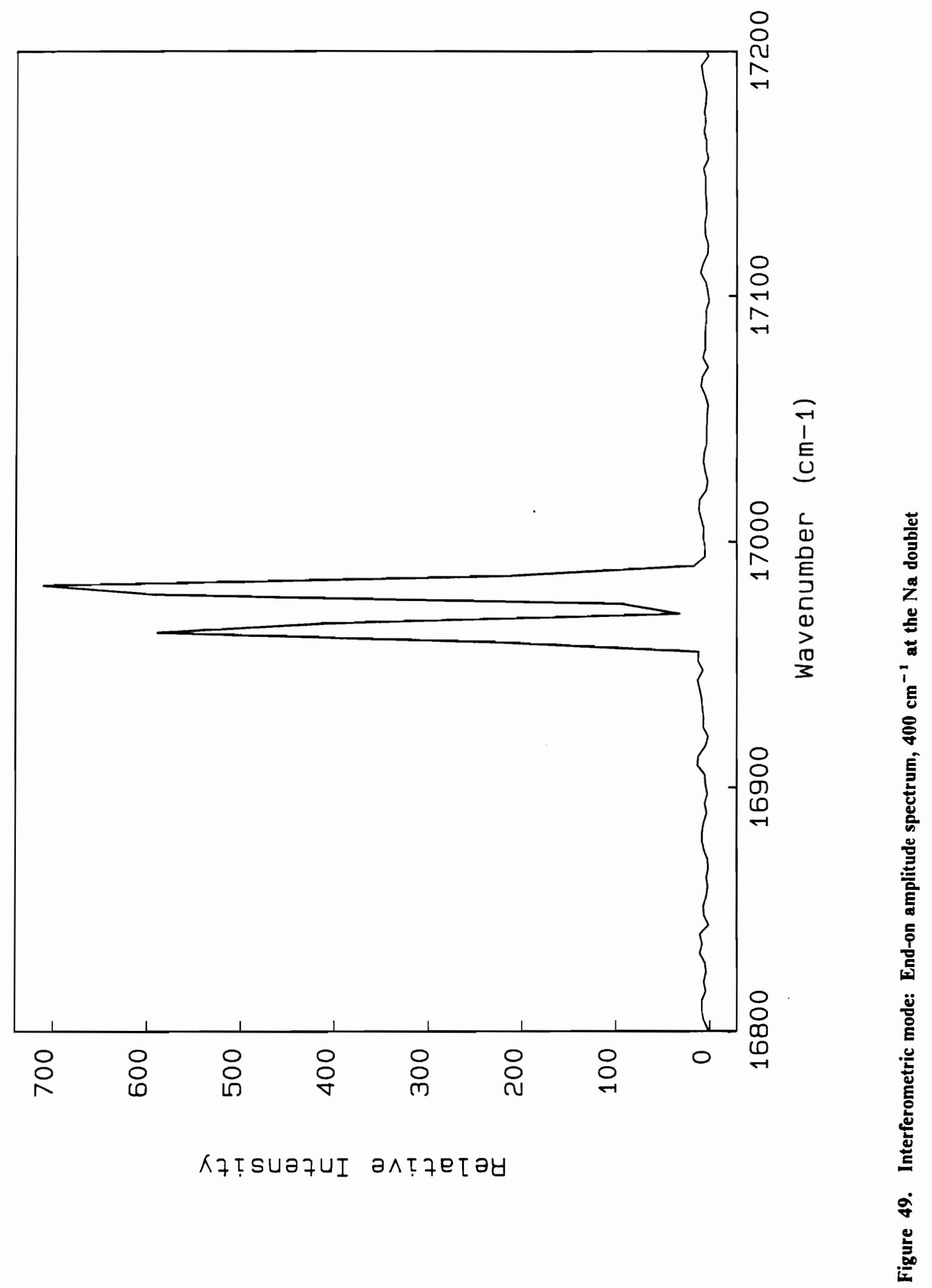


Table 24. Experimental Parameters, Interferometric Mode Comparison of End-on vs. Side-on Observation

Experimental parameters:

MIP parameters:

Sample:

Observation geometries:

Figures 44, 46, 48

Figures 45, 47, 49

MMS operating parameters:

Mode:

Transfer aperture:

Spectral bandwidth:

Pixel number:

Bandwidth center:

Alias factor:

Sampling bandwidth:

Points per

interferometer scan:

Resolution:

Resolving power:

Coadded mirror scans:

Total exposure time:

Total elapsed time:

Apodization:

$\mathrm{Na}, 100 \mu \mathrm{g} / \mathrm{mL}$

Side-on, Observation plane 1,

$0.5 \mathrm{~mm}$ from the cavity face.

End-on, Observation plane 3,

$2.0 \mathrm{~mm}$ from the plasma center

Interferometric

$5.000 \times 0.250 \mathrm{~mm}$.

$6.0 \mathrm{~nm}$

159

$590 \mathrm{~nm} ; 16,950 \mathrm{~cm}^{-1}$

[2:2]

$15,803 \mathrm{~cm}^{-1}$

$8 ; 192$.

$3.858 \mathrm{~cm}^{-1}$.

4,400

100

$256 \mathrm{sec}(4 \mathrm{~min}, 16 \mathrm{sec})$

$50 \mathrm{~min}$.

Hamming (cosine) 
Table 25. Summary: Interferometric Study of Observation Geometry, Signal and Noise Amplitudes

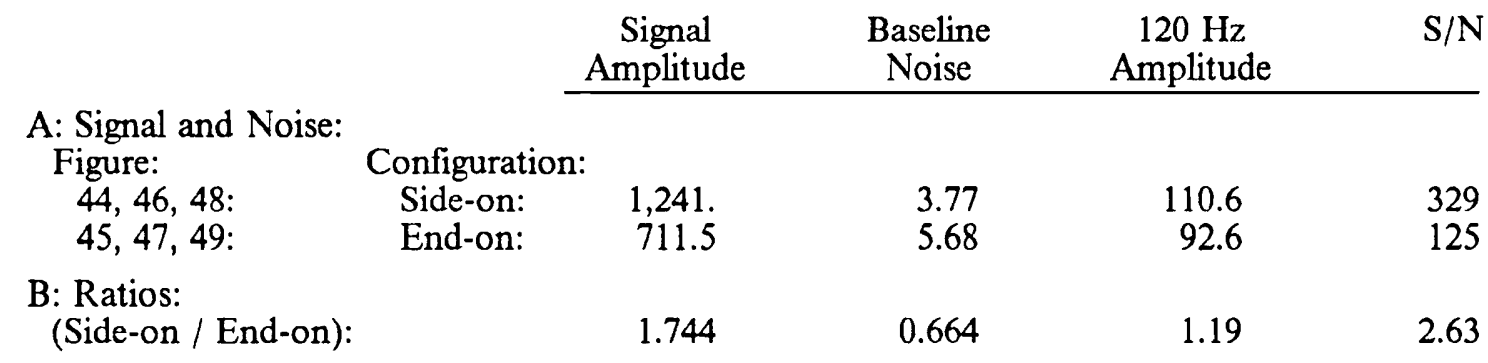


In the interferometric mode, a greater sample intensity was displayed for the side-on geometry than the end-on. These results contrast with the dispersive mode results, in which end-on geometry produced a greater analyte intensity. The dispersive mode results should be considered more reliable because of the more straight-forward operation of that mode. The interferometric mode results may be due to three factors. The first factor is the different plasma volume sampled by the larger aperture in the side-on geometry. The side-on view allowed a greater portion of the aperture to be filled with the $\mathrm{Na}$ annulus, possibly leading to a greater $\mathrm{Na}$ response. This, however, has to be weighed against the greater plasma depth sampled in the end-on configuration. A second factor is the greater volume of the cold plasma tail cone sampled by the end-on configuration. A lower average emission intensity in the plasma combined with a greater probability for selfabsorption in the plasma tail may have caused a lower detected intensity. The third factor is the ever-present instability of the interferometer alignment. This was a constant problem when analyzing signal amplitude data in the interferometric mode. Drifts in interferometer alignment caused reduced modulation in the interferogram, which in turn resulted in lower signal amplitude in the transformed spectrum. However, the trends observed in signal intensity in these experiments were generally confirmed in other experiments.

The baseline noise was 1.51 times greater for the end-on observation than the side-on. This might be anticipated. The side-on geometry observes a more homogeneous plasma environment with an established laminar flow. The end-on geometry observes a greater plasma depth, in which the sample aerosol is dried, vaporized, atomized, and excited. Furthermore, these processes may occur at different rates and depths for the plasma regions observed. Also, there was visible plasma fluctuation toward the aerosol entrance end, a transition region, in which the flow pattern through the plasma area was being established.

The $\mathrm{S} / \mathrm{N}$ for the two geometries are summarized in Table 25 . The most significant fact shown is the higher $\mathrm{S} / \mathrm{N}$ for the side-on observation. The higher side-on $\mathrm{S} / \mathrm{N}$ results from both a higher signal and a lower noise for the side-on geometry versus the end-on. Both observation geometries displayed the ubiquitous $120 \mathrm{~Hz}$ single-frequency noise, due to modulations in the microwave power supply. 


\section{Conclusion}

The Multi-Mode Spectrometer was used to probe the emission characteristics of the MIP. The dispersive mode was used to obtain an overview of plasma emissions for side-on and end-on observation. End-on survey spectra were used to quickly assign the lines to the originating species. Spatial emission characteristics were explored in the end-on geometry. Significant emission differences were found between the Ar emission core and the $\mathrm{Na}$ emission annulus. The dispersive mode was also used to obtain time-series spectra to probe the sample-take process on the frit nebulizer. These spectra revealed a nebulizer equilibration time of approximately $90 \mathrm{sec}$. The spectra also revealed that the $\mathrm{H}$ line was more affected by the introduction of $\mathrm{Na}$ than were the Ar lines.

Interferometric mode survey spectra were also obtained with the MMS. The side-on observation geometry produced a greater signal, a lower noise level, and a greater signal-to-noise ratio than the end-on geometry. No unexpected noise features due to plasma rotation, or other single-frequency noises were observed in either observation geometry.

These experiments have shown that the MMS can obtain substantial amounts of information in a variety of useful formats about a plasma source. In particular, the dispersive mode of the MMS has been shown to provide much useful information about a source in spite of the low spectral resolution. The MMS, with its operational flexibility and range of data formats, is a useful 
tool in probing the dynamic and spatially, temporally, and spectrally variable sources in atomic emission spectrometry. 


\section{Theoretical Development}




\section{Theory of Fourier Transform Spectrometry}

\section{Introduction}

This chapter discusses the aspects of Fourier transform spectrometry (FTS) relevant to the design and operation of the MMS. Many aspects of FTS theory were important throughout the development of the MMS. FTS theory was germane to the following areas: the design of the optics, the timing electronics, and the analog acquisitions electronics; many facets of the software; selection of data acquisition parameters for the interferometry data runs; and the interpretation of the interferometric spectra.

This section begins with a discussion of the Fourier transform. It then discusses the ideal FT instrument, progressively including the physical realties of a practical instrument. To shorten the leap from theory to reality, implementation of theory is discussed in terms of the MMS instrumentation.

The information on Fourier transform spectrometry and Fourier transforms in this chapter can be augmented by several sources. The body of information on Fourier transform spectrometry for the UV and visible and for (atomic) emission spectrometry is somewhat diffuse. Papers by Thorne $^{16}$ and Faires ${ }^{33}$, and many references therein, contain very good tutorial material on 
FTS-UV/Vis with implications for AES. Brault has written an excellent, but obscure, advanced tutorial on FTS as part of a short course on high resolution spectrometry in astronomy. ${ }^{39}$ A series of very good tutorials by founders of the field was put together for the Aspen International Conference on Fourier Spectroscopy, 1970.99 A number of excellent papers on high resolution FTS instrumentation for the UV/Vis were presented at the 1967 Orsay Colloquium on Fourier Transform Spectrometry. ${ }^{100-102}$

\section{Overview and Advantages of Fourier Transform}

\section{Spectrometry}

The purpose of the FT spectrometer is to is to uniquely encode each spectral element of the source so that it can be unambiguously decoded. Typically, a Michelson interferometer is used to encode, or modulate, the source radiation. The Fourier transform is the mathematical procedure used to decode the data and produce the spectrum. There are a number of advantages in using this method of encoding spectral information instead using of the spatial encoding of the dispersive spectrometer. ${ }^{33,39}$ One advantage is the need for only one detector. A second advantage is simultaneous wavelength detection over a wide spectral range. A third advantage is increased throughput. Since resolution is not a direct function of the input or output apertures of the FT spectrometer, the throughput of an FTS can often be several hundred times larger than a dispersive spectrometer. ${ }^{16}$ A fourth advantage is wavenumber or wavelength accuracy. An FT spectrometer typically incorporates a laser inside the spectrometer to assist in the encoding process. The laser provides a highly accurate internal wavelength reference, resulting in highly accurate and reproducible line positions. A fifth advantage is an easily predictable instrument line shape (ILS). A predictable and correctable ILS is an important advantage for high resolution atomic emission spectrometry, where there is interest in physical atomic line shapes and line widths. In dispersive 
spectrometers, the ILS is a function of the individual instrument, the wavelength, and the experimental conditions, so the ILS must be determined empirically for a given set of conditions. In contrast, the line shape of an FT spectrometer is easily predicted from the underlying mathematics and physical phenomena, so the effects of the ILS are easily removed in the final spectra, and the removal process can be incorporated into the FT procedure.

\section{Mathematical Foundations: The Fourier Transform}

In FTS, data is acquired in one domain, a Fourier transform is performed, and the information is interpreted in the inverse domain. A problem with this approach is the experience and instincts of the spectroscopist occurs on one side of the transform, but the useful information appears on the other side. Some degree of familiarity with the Fourier transform itself is therefore helpful in translating experimental "feel" for the data and instrumentation across the transform process. To this end, there are several very good books which help. Several books by Brigham ${ }^{61,103}$ are excellent tutorials on the discrete, fast Fourier transform. The books include excellent pictorial and theoretical developments of the Fourier transform and its ramifications in data processing. Bracewell has written several classic texts ${ }^{104,105}$ on the Fourier transform, with excellent discussions on FT applications, and a very useful pictorial library of Fourier transform pairs.

\section{The Fourier Integral}

The definition for the continuous Fourier transform pair $f(x)$ and $F(\sigma)$ is

$$
f(x)=\int_{-\infty}^{\infty} F(\sigma) e^{2 \pi i \sigma x} d \sigma
$$


and

$$
F(\sigma)=\int_{-\infty}^{\infty} f(x) e^{-i 2 \pi \sigma x} d x
$$

where

$$
e^{-i 2 \pi \sigma x}=\cos (2 \pi \sigma x)-i \sin (2 \pi \sigma x)
$$

The functions $f(x)$ and $F(\sigma)$ exist in reciprocal domains, and the variables $x$ and $\sigma$ have reciprocal dimensions, such as $\mathrm{cm}$ and $\mathrm{cm}^{-1}$, or $\mathrm{sec}$ and $\mathrm{sec}^{-1}$.

Fourier transform pairs are denoted as

$$
f(x) \rightarrow F(\sigma) \quad \text { or } \quad g(x) \rightarrow G(\sigma)
$$

An exception to this in FTS is

$$
I(x) \rightarrow B(\sigma)
$$

where $I(x)$ is the interferogram and $B(\sigma)$ is the spectrum.

\section{The Real and Imaginary Components of the Spectrum}

$F(f)$, produced by transforming $f(t)$, is composed of two orthogonal arrays, $\operatorname{Re}(f)$ and $\operatorname{Im}(f)$. This is illustrated in Figure 50. $\operatorname{Re}(f)$, the real array, contains the cosine or symmetrical components of $f(t)$. $\operatorname{Im}(f)$, the imaginary array, contains the sine or anti-symmetrical components. A function $f(t)$ which is symmetric about a central vertical plane is an even function and is composed of cosine, or real, components. A function which is symmetric about a central point is an odd function and is composed of sine, or imaginary, components. A more typical data function, which displays neither symmetry, is composed of both cosine and sine components. 

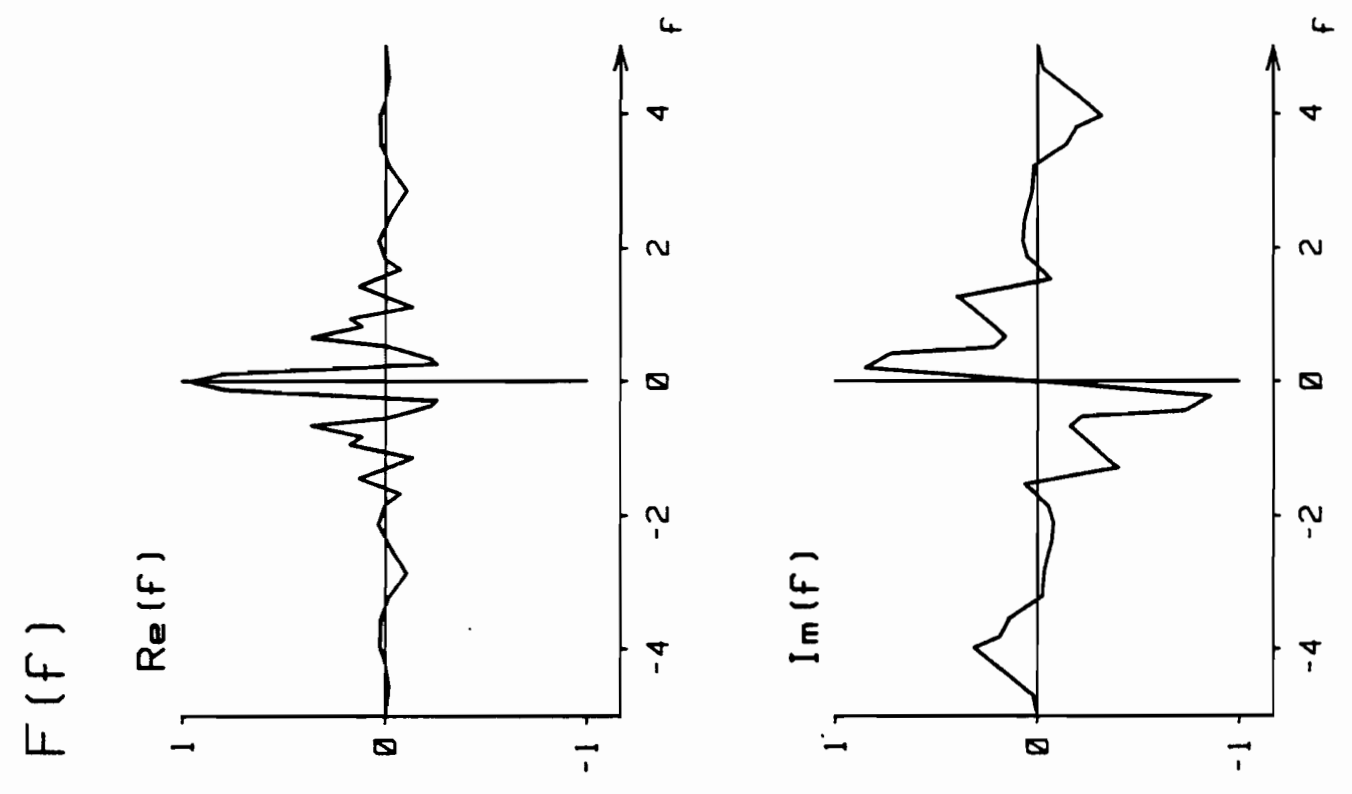

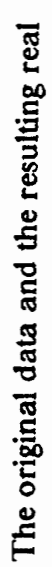

$上 \Uparrow$

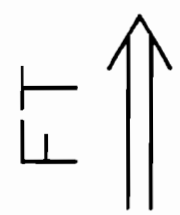

$\pm$

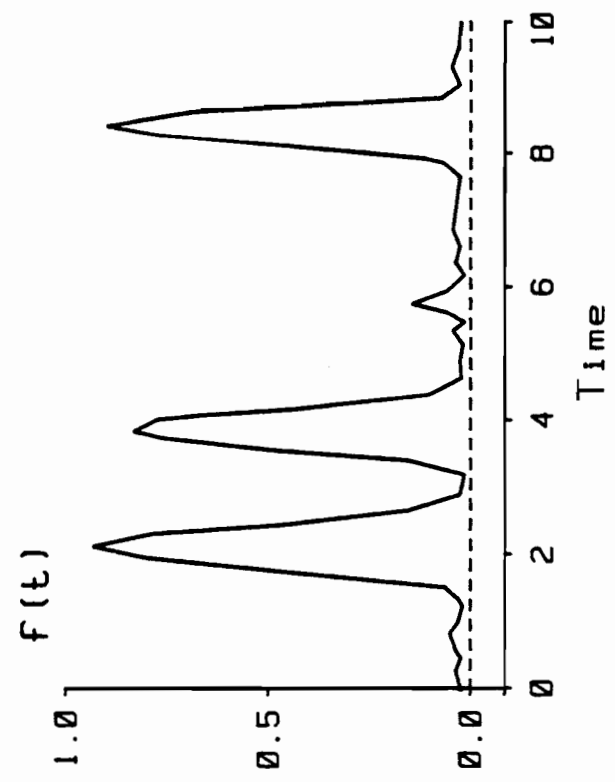

है 


\section{Amplitude and Phase Components of the Spectrum}

$\operatorname{Re}(f)$ and $\operatorname{Im}(f)$ comprise a rectangular representation of the frequency composition of $f(t)$. A polar representation can be obtained by calculating the amplitude and phase spectra, $A m p(f)$ and $P h a(f)$, where

$$
A m p(f)=\sqrt{\operatorname{Re}(f)^{2}+\operatorname{Im}(f)^{2}}
$$

and

$$
P h a(f)=\arctan \frac{\operatorname{Im}(f)}{\operatorname{Re}(f)}
$$

This process is illustrated for hypothetical data in Figure 51

The relationships between $\operatorname{Re}(f), \operatorname{Im}(f), A m p(f)$, and $P h a(f)$ for a single frequency are shown in Figure 52. Figure 53 illustrates the concept for an entire spectrum, from $f_{0}$ to $f_{\max }$. At a given frequency, $\operatorname{Amp}(f)$ is the total signal magnitude. $\operatorname{Re}(f)$ and $\operatorname{Im}(f)$ are the portions of $A m p(f)$ projected into the real and imaginary planes. $P h a(f)$ is the angle by which $A m p(f)$ is rotated out of the real plane.

Shifting the original data function $f(t)$ in time produces a linear phase shift in $F(f)$. In Figure 53 , the spiral in the data from $f_{0}$ to $f_{\max }$ shows the effect on the data. The total frequency content of $f(t)$ is unchanged by translation. Translating $f(t)$ does, however, change its symmetry. Therefore, the phase angles in $P h a(f)$ are changed, thus changing the distributions of $A m p(f)$ between $\operatorname{Re}(f)$ and $\operatorname{Im}(f)$.

\section{Properties of the Fourier Transform}

The properties of the FT most useful to FTS $39,106,104$ are summarized in equations 19 to 22 . These are the properties which most assist in translating experience between reciprocal domains.

- Addition: $\quad f(x)+g(x) \rightarrow F(\sigma)+G(\sigma)$ 

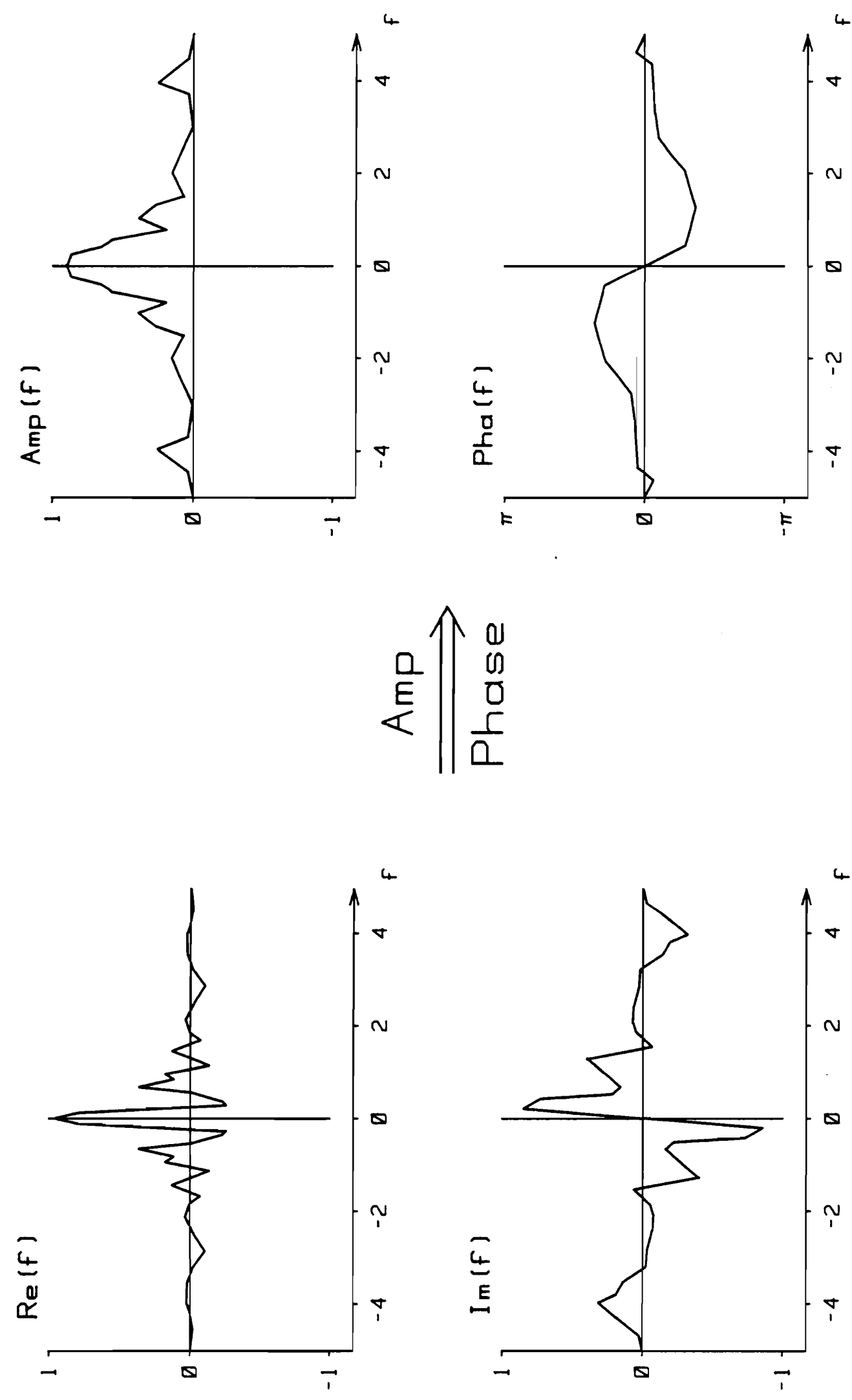

$\frac{a}{E} \Uparrow_{\frac{1}{0}}^{0}$
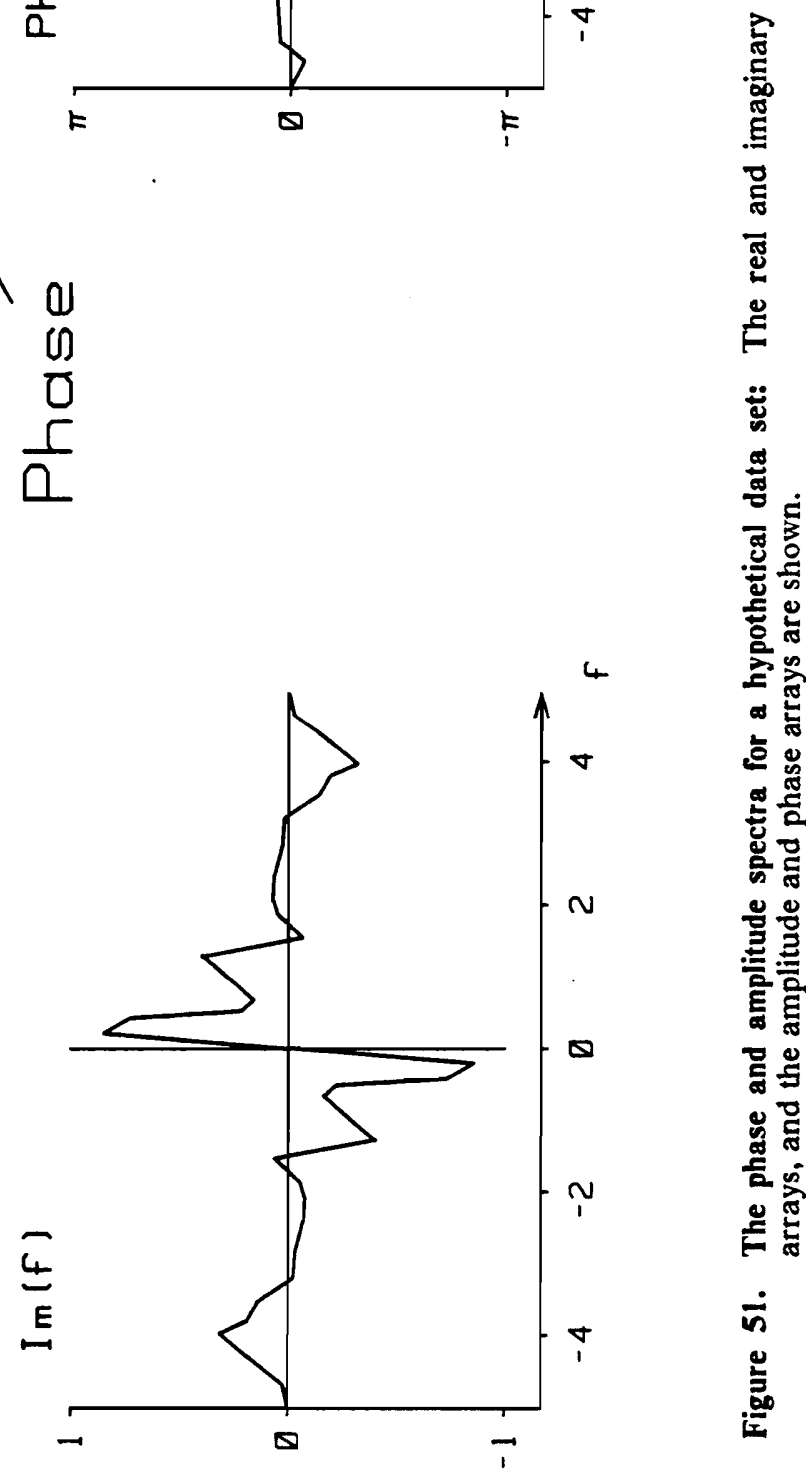


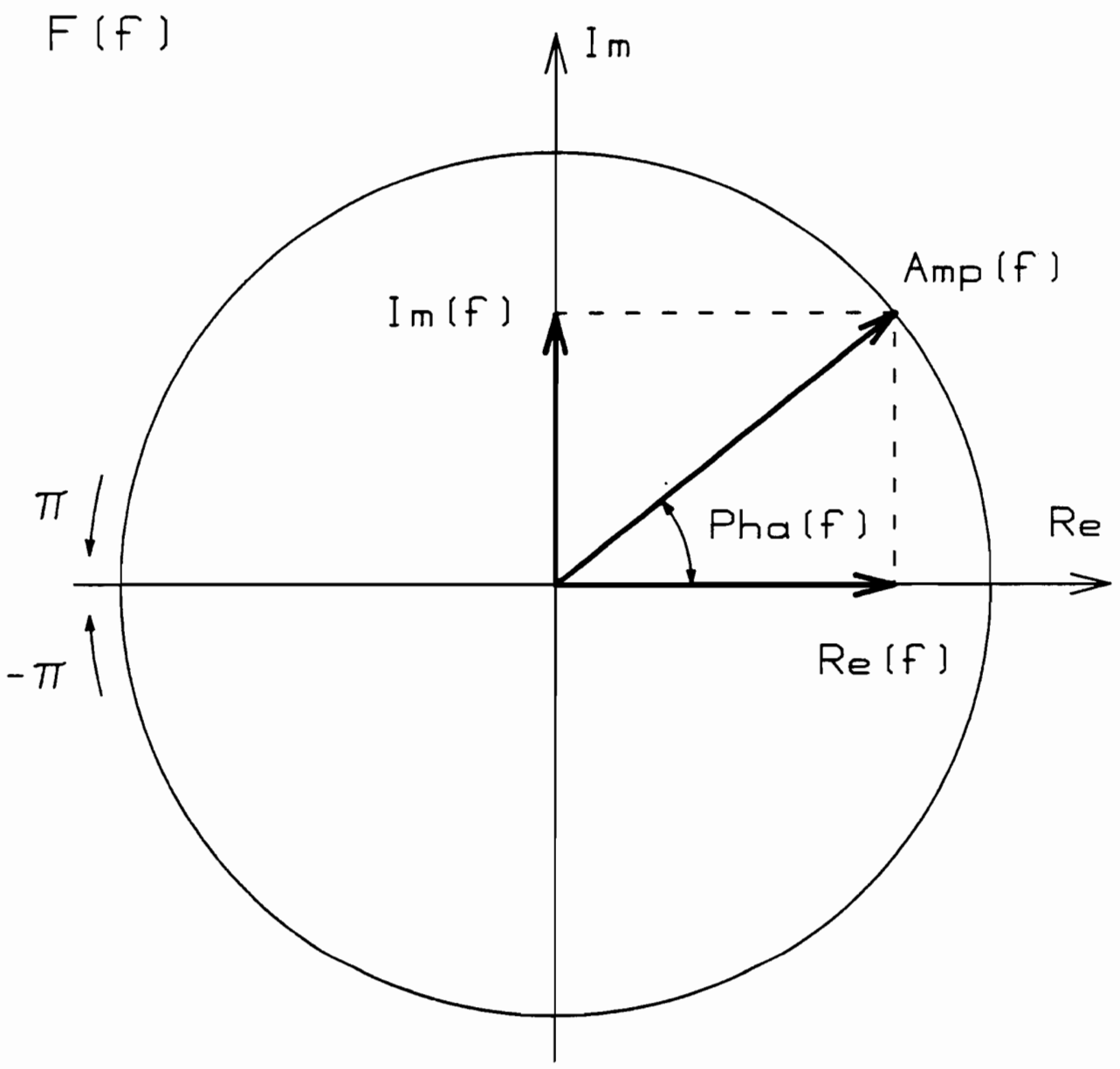

Figure 52. The Real, Imaginary, Amplitude, and Phase values for a single frequency 


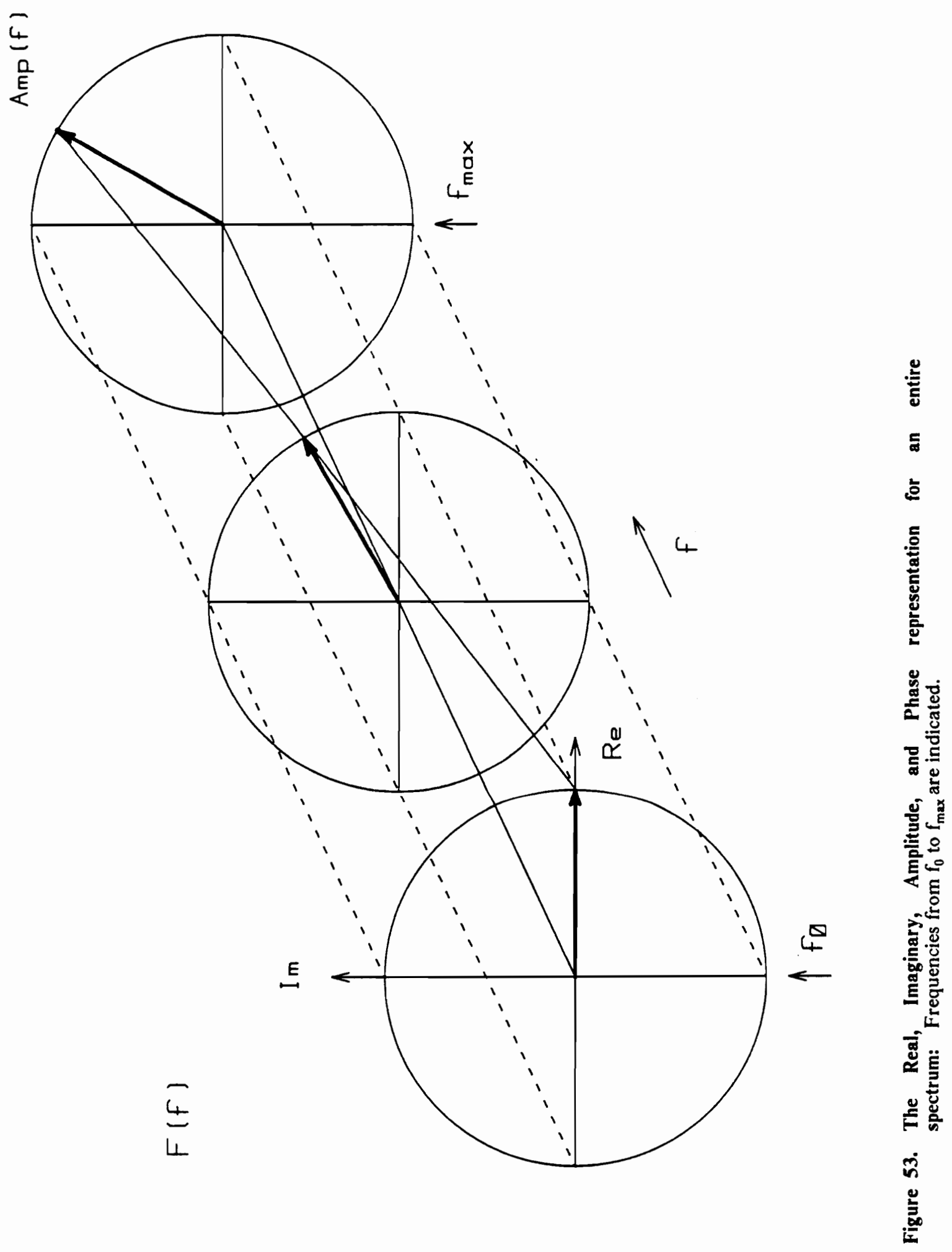


The addition theorem states the simple but significant condition that adding data in one domain is equivalent to adding data in the reciprocal domain. Because of this, data can be added in either domain to improve the $\mathrm{S} / \mathrm{N}$, or data may be subtracted to remove background features.

$$
\text { - Similarity: } \quad f(a x) \rightarrow \frac{1}{|a|} F\left(\frac{\sigma}{a}\right)
$$

The similarity theorem states that if $f(x)$ and $F(\sigma)$ are an FT pair, expanding the scale of $f(x)$ compresses the scale of $F(\sigma)$. Practical examples in FTS include the following: (1) Reducing the rise time of the A/D filters in the interferogram domain increases the frequency response in the spectral domain. (2) Widening the apodization function in the interferogram domain narrows the corresponding line shape in the spectral domain.

$$
\text { - Shift: } \quad f(x-a) \rightarrow e^{-i 2 \pi \sigma a} F(\sigma)
$$

The shift theorem describes the result of shifting a data set $f(x)$ by an amount an $a$. This effect was described above and illustrated in Figure 53. Shifting $f(x)$ causes a linear phase shift in $F(\sigma)$. In Figure 53, this effect is represented as the uniform spiral from $f_{0}$ to $f_{\max }$. The greater the shift in $f(x)$, the tighter the spiral in $F(\sigma)$. In FTS, phase correction reduces this and other phase distortion. Phase correction causes $A m p(f)$ to be rotated back into the $\operatorname{Re}(f)$ plane by making Pha(f) approximately 0.

$$
\text { - Convolution: } \quad f(x) \cdot g(x) \rightarrow F(\sigma)^{*} G(\sigma)
$$

The convolution theorem is one of the most useful in FTS, helping to translate a wide range of effects between domain. This theorem states that multiplication in one domain is equivalent to convolution in the other. By convention, ${ }^{104}(\cdot)$ indicates multiplication, and $\left({ }^{*}\right)$ indicates convolution. Convolution, particularly in the time domain, is synonymous with filtering, scanning, smearing, or smoothing. Practical examples of convolution in FTS include the following: (1) Analog filtering in the interferogram is a convolution operation. It is therefore is equivalent to multiplying the frequencies in $B(\sigma)$ by the Fourier transform of the filter response function. (2) Apodization is an example of convolution. The purpose of apodization is to control the profile of the instrument line shape and its effect on the final presentation. For apodization, the 
interferogram $I(x)$ is multiplied by a broad windowing function. In the reciprocal domain, this is equivalent to convoluting or scanning $B(\sigma)$ with the $\mathrm{FT}$ of the windowing function.

\section{The Discrete Fourier Transform}

The continuous Fourier transform cannot be calculated by digital computer, but must be approximated by the discrete Fourier transform (DFT). The discrete Fourier transform is defined by the following pair of equations

$$
\mathrm{B}(\sigma)=1 / N \sum_{x=0}^{N-1} I(x) e^{-i 2 \pi(\sigma / N) x} \quad \sigma=0,1, \ldots, N-1
$$

and

$$
I(x)=\sum_{\sigma=0}^{N-1} \mathrm{~B}(\sigma) e^{i 2 \pi(\sigma / N) x} \quad x=0,1, \ldots, N-1
$$

Typically, the discrete FT is computed using the fast Fourier transform (FFT) algorithm. The FFT, a very efficient algorithm for calculating the discrete Fourier transform, reduces the number of actual computer operations by exploiting symmetries of the computation matrices. The FFT algorithm, published by Cooley and Tukey ${ }^{107}$ in 1965 , was and continues to be a major factor in making FTS a practical technique. 


\section{Basic FTS Theory}

\section{The Ideal Michelson Interferometer and Monochromatic Light}

A good starting point for FTS theory is the Michelson interferometer and its effect on a monochromatic point source. In FTS, source light is typically modulated by passing it through a Michelson interferometer and changing the optical path difference between the two arms. Figure 54 shows a schematic of an ideal Michelson interferometer. The effect of the interferometer on a monochromatic point source is shown in Figure 55. Referring to Figures 54 and 55, if the optical pathlengths in the two arms of the interferometer are equal, the optical path difference is zero, so the two beams constructively interfere. As the optical path difference is changed by moving the mirror, the light goes through the series of constructive and destructive interferences shown in Figure 55. Because the spacing of the interference pattern depends directly upon the wavelength of the light, each wavelength produces a unique interference pattern. If the source is polychromatic, the interference patterns of the individual wavelengths are superposed, and then decoded by the Fourier transform.

The intensity at the detector shown in Figure 55, can be expressed as

$$
I^{\prime}(x)=\mathrm{B}(\sigma)[1+\cos (2 \pi \sigma x)]
$$

where $I^{\prime}(x)$ is the intensity at the detector as a function of optical path difference, $B(\sigma)$ is the spectral intensity of the source, $x$ is optical path difference $(\mathrm{cm})$, and $\sigma$ is the wavenumber of the light $\left(\mathrm{cm}^{-1}\right)$. Recall that $\sigma=1 / \lambda$.

Equation 25 can be rearranged to give

$$
I^{\prime}(x)=\mathrm{B}(\sigma)+\mathrm{B}(\sigma) \cos (2 \pi \sigma x)
$$

where $B(\sigma)$ is the unmodulated DC portion of the interferogram, and $B(\sigma) \cos (2 \pi \sigma x)$ is the modulated AC portion of the interferogram. The $\mathrm{DC}$ level due to $\mathrm{B}(\sigma)$ is equal to $I_{a v e}$ in Figure 


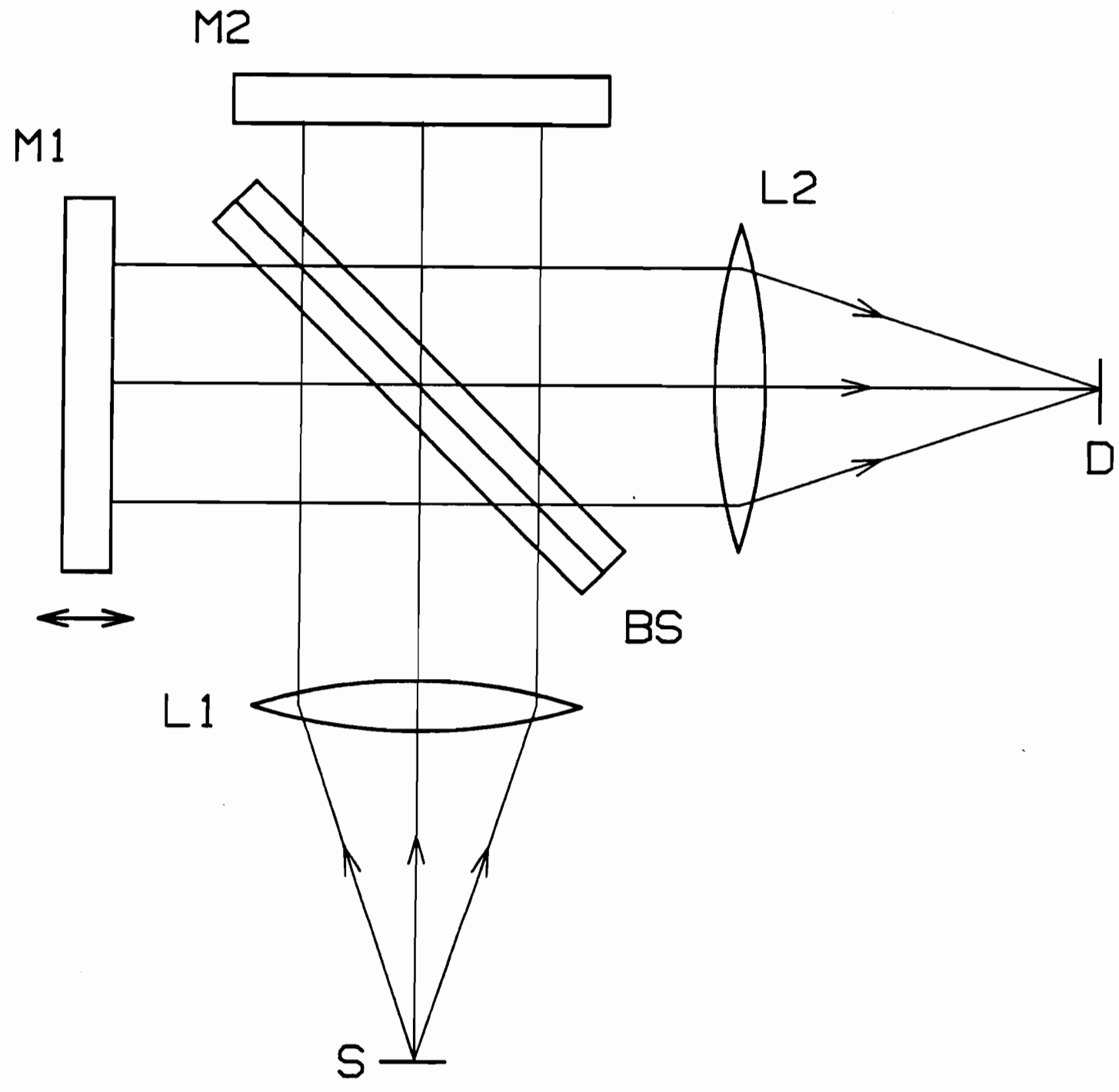

Figure 54. Schematic of a Michelson interferometer: S: source. L1: collimating optic. L2: focusing optic. M1: moving mirror. M2: stationary mirror. BS: beamsplitter. D: detector. 


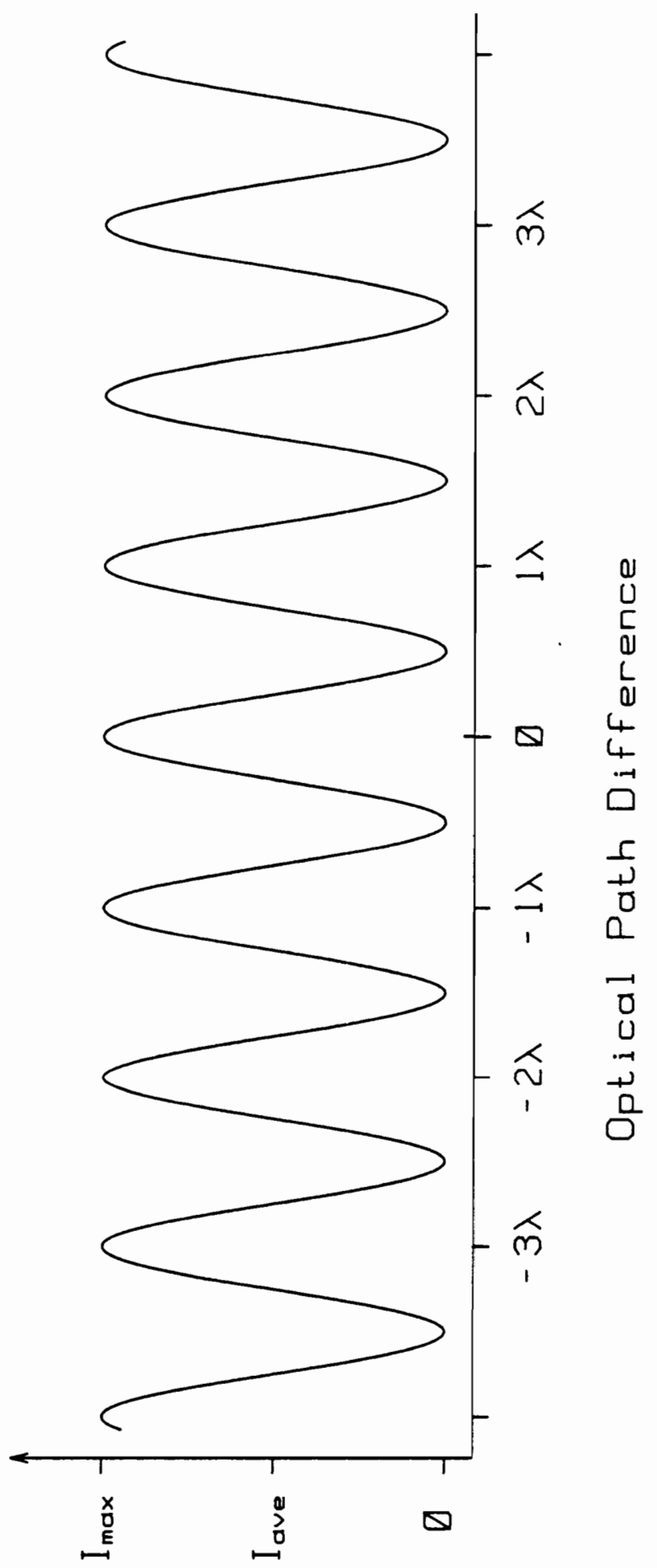



ศิ7 เ งบลษบI 
55. In practice, this constant is removed from the interferogram by hardware and/or software before the Fourier transform is performed, since it contains no wavenumber information. The modulated portion,

$$
I(x)=\mathrm{B}(\sigma) \cos (2 \pi \sigma x)
$$

is the generally recognized interferogram. The spectrum can be recovered from the ideal interferogram by performing the cosine transform,

$$
\mathrm{B}(\sigma)=I(x) \cos (2 \pi \sigma x)
$$

\section{Polychromatic Light}

By considering the effects of a physically more interesting polychromatic source, the integral for the interferogram, equation 25 , becomes

$$
I^{\prime}(x)=\int_{-\infty}^{\infty} \mathrm{B}(\sigma) d \sigma+\int_{-\infty}^{\infty} \mathrm{B}(\sigma) e^{-i 2 \pi \sigma x} d \sigma
$$

and equation 26 becomes

$$
I(x)=\int_{-\infty}^{\infty} \mathrm{B}(\sigma) e^{-i 2 \pi \sigma x} d \sigma
$$

In equation $29, \int_{-\infty}^{\infty} \mathrm{B}(\sigma) d \sigma$ represents the integrated spectral intensity, which must be removed prior to transformation. Equation 30 expresses the spectrally useful interferogram. Spectral recovery is done through the inverse transform

$$
\mathrm{B}(\sigma)=\int_{-\infty}^{\infty} I(x) e^{i 2 \pi \sigma x} d x
$$


The exponential notation in these three equations recognizes that a real interferogram is asymmetric, exhibiting phase distortions due to several effects within the interferometer.

\section{The Michelson Interferometer as a Frequency Shifter}

In addition to being a modulator, the interferometer can be considered a frequency shifter or converter. As the path difference is changed at a rate of $v(\mathrm{~cm} / \mathrm{sec})$, each optical frequency $v$ is uniquely down-shifted to an audio frequency $f$, where $f=\frac{\mathrm{v} v}{c}=\mathrm{v} \sigma$. The frequency conversion factor is $\left(\frac{v}{c}\right)$, the mirror velocity divided by the speed of light.

Replacing the variables $(x, \sigma)$ with $(f, t)$, the interferogram and the spectrum can be expressed as

$$
I(t)=\int_{-\infty}^{\infty} \mathrm{B}(f) e^{-i 2 \pi f t} d f
$$

and

$$
\mathrm{B}(f)=\int_{-\infty}^{\infty} I(t) e^{i 2 \pi f t} d t
$$

Several practical considerations for the FT spectrometer follow from this interpretation. First, the detector, filters, and electronics must typically respond over a frequency range of from 1 $\mathrm{kHz}$ to $10 \mathrm{kHz}$. Second, the mirror movement mechanism must be capable of producing a constant mirror velocity so that the resultant audio frequencies falls within the frequency response range of the detection system. Third, because of the Nyquist criterion, the data acquisition system must be capable of acquiring data at a rate twice that of the highest frequency produced by the interferometer. In the UV and visible $v$ is approximately $10^{15} \mathrm{~Hz}$, so for FTS- UV/Vis, $f$ is typically 1 to $10 \mathrm{kHz}$, and $\mathrm{v}$ is 0.01 to $0.1 \mathrm{~cm} / \mathrm{sec}$. 


\section{Physical Limitations}

\section{Real Optical Components}

In a real interferometer, the beamsplitter, mirrors, and lenses are not total efficient. Ideally, the beam splitter should provide, at all wavelengths, $50 \%$ transmission and $50 \%$ reflection with no absorption. The mirrors and lenses should provide $100 \%$ reflection and transmission. Including these factors, the Fourier integral for the interferogram becomes

$$
I(x)=\int_{-\infty}^{\infty} \mathrm{B}(\sigma) \eta_{o} \eta_{b} e^{-i 2 \pi \sigma x} d \sigma
$$

The terms $\eta_{0}$ and $\eta_{b}$ represent the efficiencies of the optics and beamsplitter, respectively. $\eta_{b}=4 R_{b} T$, where $R_{b}$ and $T$ are the reflectance and transmittance of the beam splitter. $\eta_{o}$ and $\eta_{b}$ are usually not expressed, but their presence is always implicit. Deviations of $\eta_{0}$ and $\eta_{b}$ from values of 1 result in reduced modulations and thus reduced spectral intensities. Changes in the refractive index of the beamsplitter with wavelength contribute to phase distortions in the interferogram.

\section{Finite Path Difference and Resolution}

An ideal spectrum, $B(\sigma)$, could be recovered from an infinitely long interferogram, $I(x)$. But a real interferometer has a physical limit to its optical path difference, so there must be some compromise in $B(\sigma)$. The compromise is the instrument line shape and the resolution in $B(\sigma)$.

If $L$ is the maximum optical path difference, the range of pathlength differences is $(-L \leq x \leq+L)$, and the inverse transform of $I(x)$ becomes 


$$
\mathrm{B}(\sigma)=\int_{-L}^{+L} I(x) e^{12 \pi \sigma x} d x
$$

The Fourier transform pair $\Pi(x)$ and $\operatorname{sinc}(\sigma)$ is shown in Figure 56, where $\operatorname{sinc}(\sigma) \equiv \frac{\sin (\pi \sigma)}{\pi \sigma}$. Acquiring data over a finite path difference is equivalent to acquiring an infinitely long interferogram and then multiplying it by $\Pi(x)$. This is equivalent to convoluting $B(\sigma)$ with $\operatorname{sinc}(\sigma)$, or $\left[I(x) \cdot \Pi(x) \rightarrow B(\sigma)^{*} \sin c(\sigma)\right]$. Therefore, the sinc function is the basic ILS, or scanning function, of an FT spectrometer. It is equivalent to the slit function of a dispersive spectrometer, and is the response of an FT spectrometer to a monochromatic line. As shown in Figure 56, the zero-crossings of the sinc function occur at intervals equal to $1 / 2 L$ wavenumbers. Therefore, the theoretical resolution of the spectrometer is conventionally defined as 72,39

$$
\delta \sigma=1 / 2 L
$$

The full-width at half-maximum of the sinc function is $1.207 \cdot \delta \sigma$. The abrupt discontinuities of the $\Pi(x)$ function at $\pm L$ cause the ringing in the wings of the sinc function is due to the abrupt discontinuities of the rect function. The ringing can be reduced through apodization.

\section{Finite Source and Detector Apertures}

\section{Introduction}

The effects and the limits of finite source and detector apertures must be considered since real sources and real detectors have some physical aperture size, and not the ideal point geometry assumed so far. The allowable sizes of these apertures results in the throughput advantage, but there is a limit to their size. Other consequences of aperture geometry include effects on the ILS and the wavenumber calibration of the FT spectrometer. Typically, circular shapes are used 


\section{$\Pi(t)$}

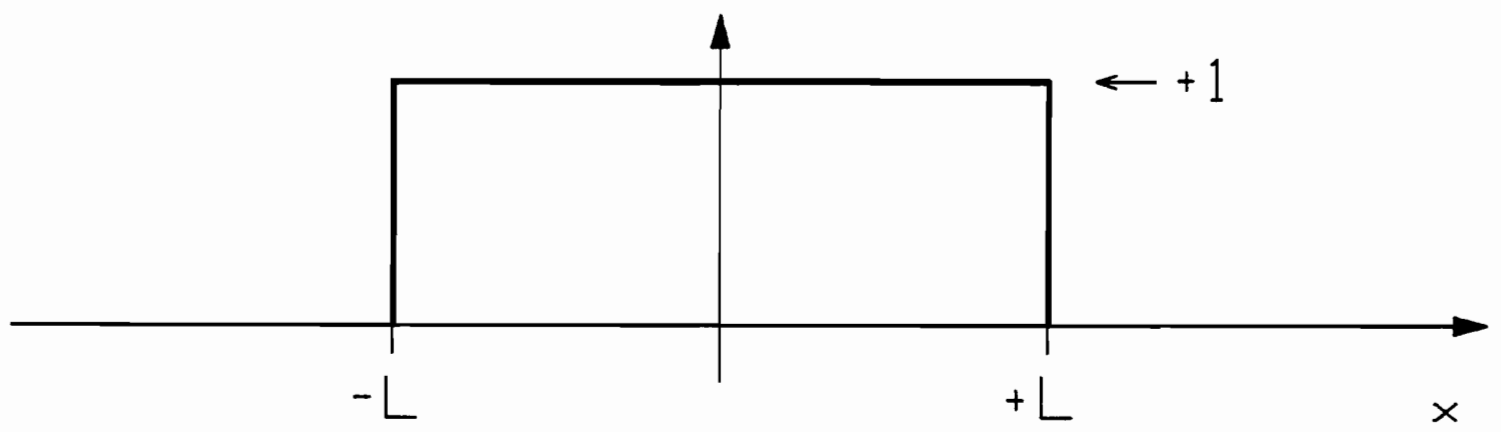

$\sqrt{1} T$

$\operatorname{sinc}(f)$

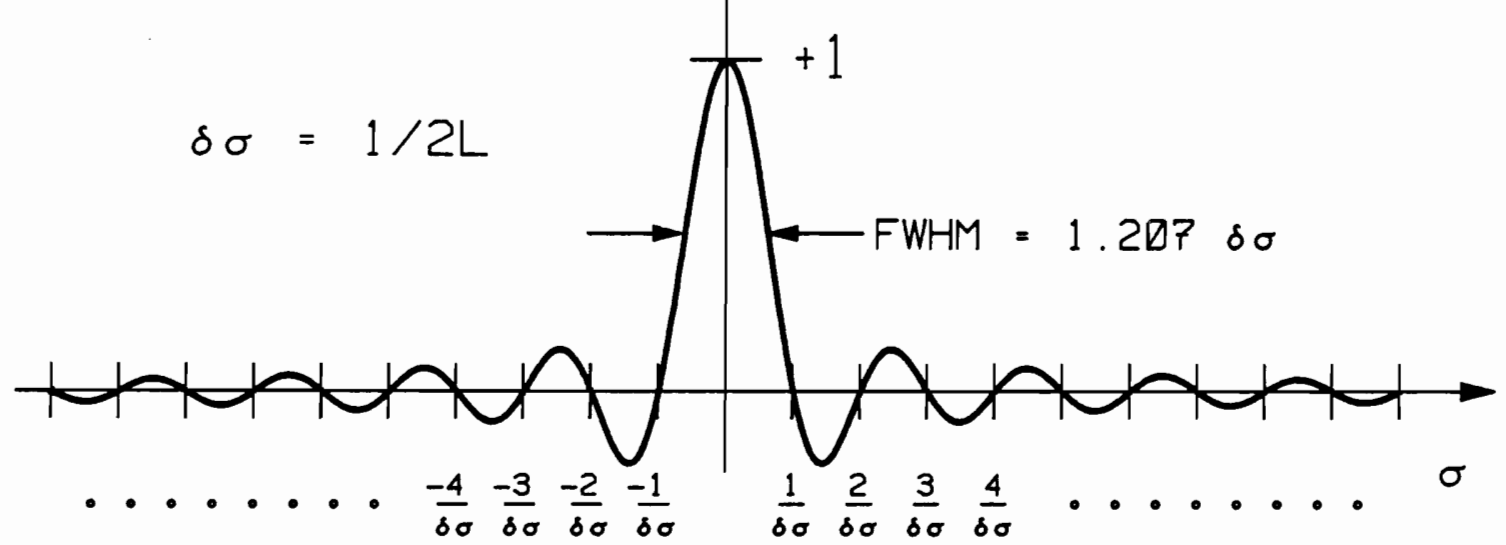

Figure 56. Maximum path difference and resolution: The mirror scanning function, $\Pi(x)$, and its Fourier transform, $\sin c(\sigma)$, are shown. 
because of the circular symmetry along the optical axis of the interferometer. Due to the symmetry across the beamsplitter, the source and the detector are mirror images of each other, and their effects can be considered together.

Entrance and exit aperture effects were particularly important to the design of the MMS optical system. In the MMS, the effects discussed here occur at the transfer aperture rather than at the detector plane in a typical interferometer. The phenomena had to be considered carefully to insure proper interfacing of the interferometric and the dispersive optical subsystems. In addition, the rectangular slit of the transfer aperture disrupted the typical circular symmetry along the optical axis.

\section{Haidinger Fringes: The Basic Phenomenon}

The basic FTS equations assumes that the light in the interferometer forms a collimated plane wave travelling parallel to the optical axis. With an extended source however, off-axis points in the source give rise to rays which travel at angle $\alpha$ to the optical axis. Because of this, the interference pattern known as Haidinger fringes is formed at the focal plane of the focusing optics. The pattern is shown in Figure 57. The vertical axis of this Figure corresponds to the optical axis of the interferometer, and the horizontal plane of the Figure corresponds to the plane of the detector. It has been shown ${ }^{39}$ that the radii of the fringes are given by

$$
r_{f}=F \sqrt{\frac{2 n}{x_{i} \sigma_{i}}},
$$

where $r_{f}$ is the radius of an intensity minimum, $\sigma_{l}$ is the wavenumber of interest, $x_{i}$ is the optical path difference, $F$ is the focal length of the focusing optic, and $n$ is the number of the fringe. Figure 58 shows the relationship between the $F, \alpha$, and $r$, and that angles through the interferometer are mapped into rings at the detector. As $x_{i}$ and the resolution become larger, or as $\sigma_{i}$ larger greater, $r_{f}$ becomes smaller. In high resolution FT-UV/Vis spectrometry, both $x_{i}$ and $\sigma_{i}$ can become much larger than in the IR. 


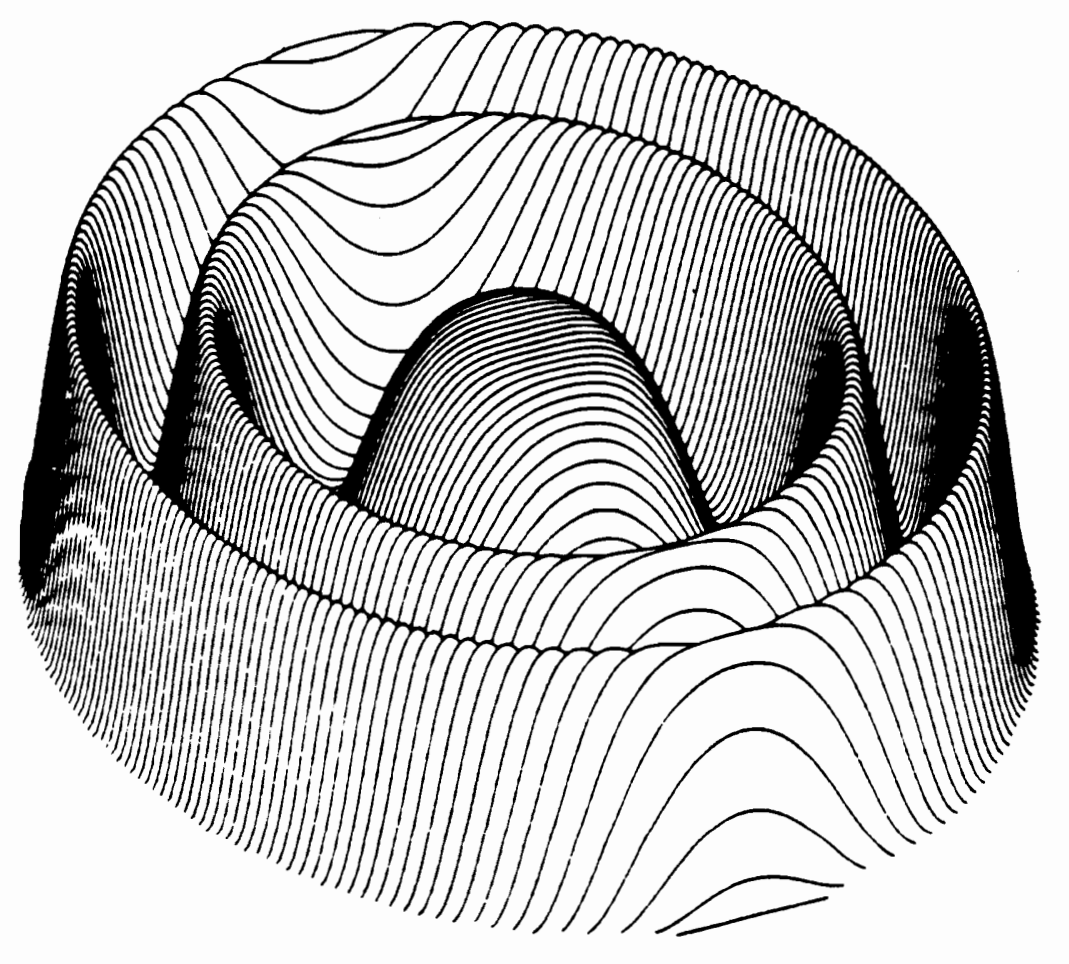

Figure 57. Haidinger fringes: the spatial intensity profile at the detector plane: The vertical axis of the Figure corresponds to the optical axis of the interferometer. The horizontal plane corresponds to the plane of the detector. From reference 108. 


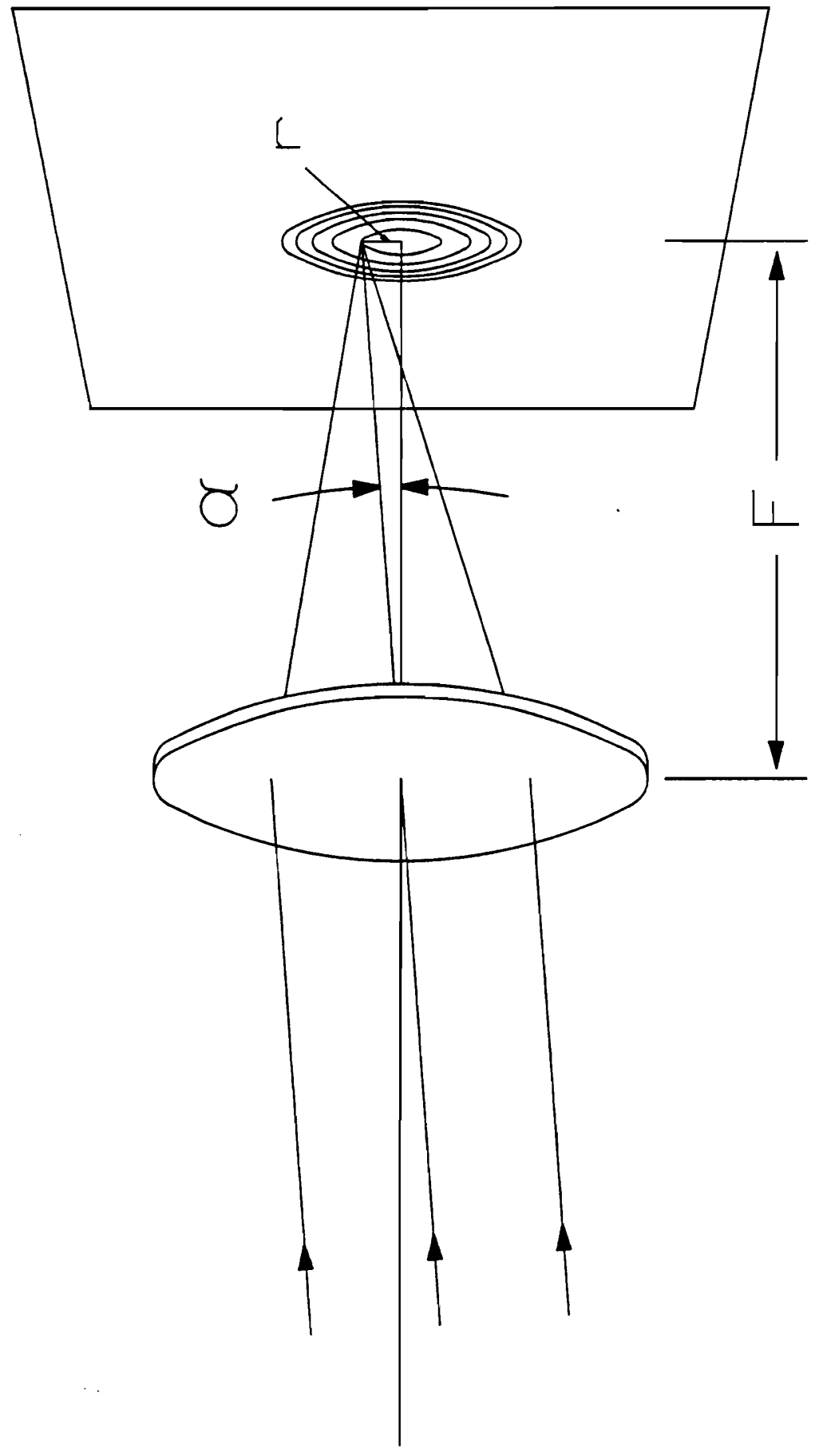

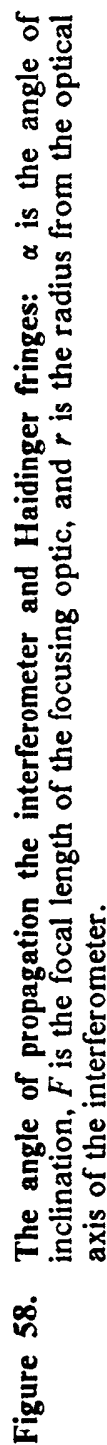


Haidinger fringes result because light propagating at angle $\alpha$ travels a shorter path difference than light parallel to the optical axis. The actual path difference is $[x \cdot \cos (\alpha)]$. More intuitively, the light at angle $\alpha$ is only modulated by the component of the mirror velocity parallel to the light axis, or $[\mathrm{v} \cdot \cos (\alpha)]$.

Haidinger fringes comprise an intensity pattern perpendicular to the optical axis. For a path difference of zero, the central fringe is infinitely large. As the path difference increases, the fringe pattern collapses toward the center, and the central point undulates as a cosine. The undulation of the central point is describe by Figure 55. Also as the path difference increases, the pattern shown in Figure 57 decreases in size as $1 / \sqrt{x_{0}}$

\section{The Aperture Size Limits}

The purpose of the interferometer is to produce maximum overall modulation and contrast at the detector, so it is necessary to restrict the field of view to the central fringe. Because $r_{f}$ and $r_{d}$ must be selected for a given set of conditions, a limiting value is chosen where $\sigma_{i}=\sigma_{\max }$ and $x_{i}=L=\frac{\delta \sigma}{2}$, and where $\delta \sigma$ is the interferometer resolution. The number of the fringes, $n$, is chosen to produce the maximum fringe amplitude for the highest wavenumber at the greatest path difference. It can be shown through further proof that typically $n=1 / 2.3^{39}$

\section{Instrumental Consequences of Finite Apertures}

The Throughput Advantage:One effect of large source and detector apertures is the throughput advantage. The throughput advantage arises because of both a large acceptance angle and a large detector area relative to a dispersive spectrometer. The solid angle of acceptance at the detector, $\Omega_{d}$, is given as

$$
\Omega_{d}=\frac{2 \pi}{x_{i} \sigma_{i}} n=\pi \alpha^{2}
$$


The throughput of the interferometer, $E_{i}$, is

$$
E_{i}=A_{d} \cdot \Omega_{d}=\pi r_{d}^{2} \Omega_{d}
$$

where $A_{d}$ is the area of the detector. As stated above, FT spectrometers can have a throughput advantage of several hundred in the UV/Vis. ${ }^{16}$

Wavenumber Scale Change:A second effect of the apertures is a wavenumber scale change..$^{99}$ This arises, light propagating through the interferometer at angle $\alpha$ is modulated at a lower frequency than light of the same wavelength propagating parallel to the optical axis. The correction factor for this effect is

$$
\sigma_{c}=\sigma_{o}\left(1-\frac{\Omega}{4 \pi}\right)
$$

where $\sigma_{c}$ is the corrected wavenumber, and $\sigma_{o}$ is the observed wavenumber.

IIS Distortion:A third aperture effect is an attenuation of $I(x)$ as the path difference increases. The attenuation results as the central mound in Haidinger fringes becomes smaller with larger path differences. The total modulation inside of the detector area then becomes correspondingly lower. This effect is known as self-apodization. The attenuation of $I(x)$ is

$$
I_{o}(x)=I(x) \cdot \sin c\left[\frac{\sigma_{t} x \Omega}{2 \pi}\right]
$$

where $I_{o}(x)$ is the observed interferogram. In other words, $I(x)$ is multiplied by a sinc function, but one that varies with $\sigma_{l}$. Multiplication in the interferogram, of course, implies convolution in the spectrum. Therefore, $B(\sigma)$ is convoluted with a wavenumber-dependent $\Pi(\sigma)$ function. Convolution in the wavenumber domain broadens the ILS, but again it is a predictable phenomenon. 


\section{Interaction of Haidinger fringes and the MMS Optical System}

Figure 59 is a diagrammatic study of the interaction of Haidinger fringes with the important focal plane response functions within the MMS. The Figure also includes the detector aperture response of a conventional interferometer for comparison. A convenient place to start is with the interaction of Haidinger fringes (a) and the conventional interferometer detector aperture response function (b). As described above, Haidinger fringes are produced by the interferometer and projected onto the detector plane. (b) represents the detector with diameter $d_{I}$ and unit response across its surface, as indicated by the height of the volume. Following the discussion above, the

relative size of $d_{1}$ was chosen for the following conditions: $\sigma_{i}=\sigma_{\max }, x_{i}=L=\frac{\delta \sigma}{2}$, and $n=1 / 2$ The response of the detector to the fringe pattern at this extreme in the mirror stroke can be found by doing a point-for-point multiplication between Haidinger fringes and the surface of (b), and then integrating the results. The interaction of the edges of (b) with the sloping sides of the central cone of (a) causes the reduced modulation at extreme mirror stoke.

The same type of analysis can be done for the MMS. Feature (c) in the Figure represents the MMS transfer aperture. $h_{A}$ and $w_{A}$ represent the height and width of the aperture. The height of (c) in the Figure again represents unit response across the area. (c) is not drawn to any particular scale. As can be seen, the point-for-point multiplication of the transfer aperture with the fringe pattern can lead to a significantly different result than with the circular geometry of (b).

Within the MMS then, the transfer aperture essentially selects a portion of the fringe pattern. The polychromator then projects this multiplied volume onto the detector array. The geometric response function of a single detector pixel is shown in (d). The height of the pixel is represented by $h_{P}$ The response across the width of the pixel, $w_{P}$, is a trapezoid. This shape is explained in the photodiode array chapter. Again, (d) is not drawn to any particular scale.

The shapes of the aperture response function and the pixel response function have considerable impact on optical throughput and spectral discrimination capabilities of the MMS. Since the width of the pixel response function, $w_{P}$, is usually less than the width of the transfer aperture, $w_{A}$ the pixel response function usually limits the throughput in the horizontal plane. The 


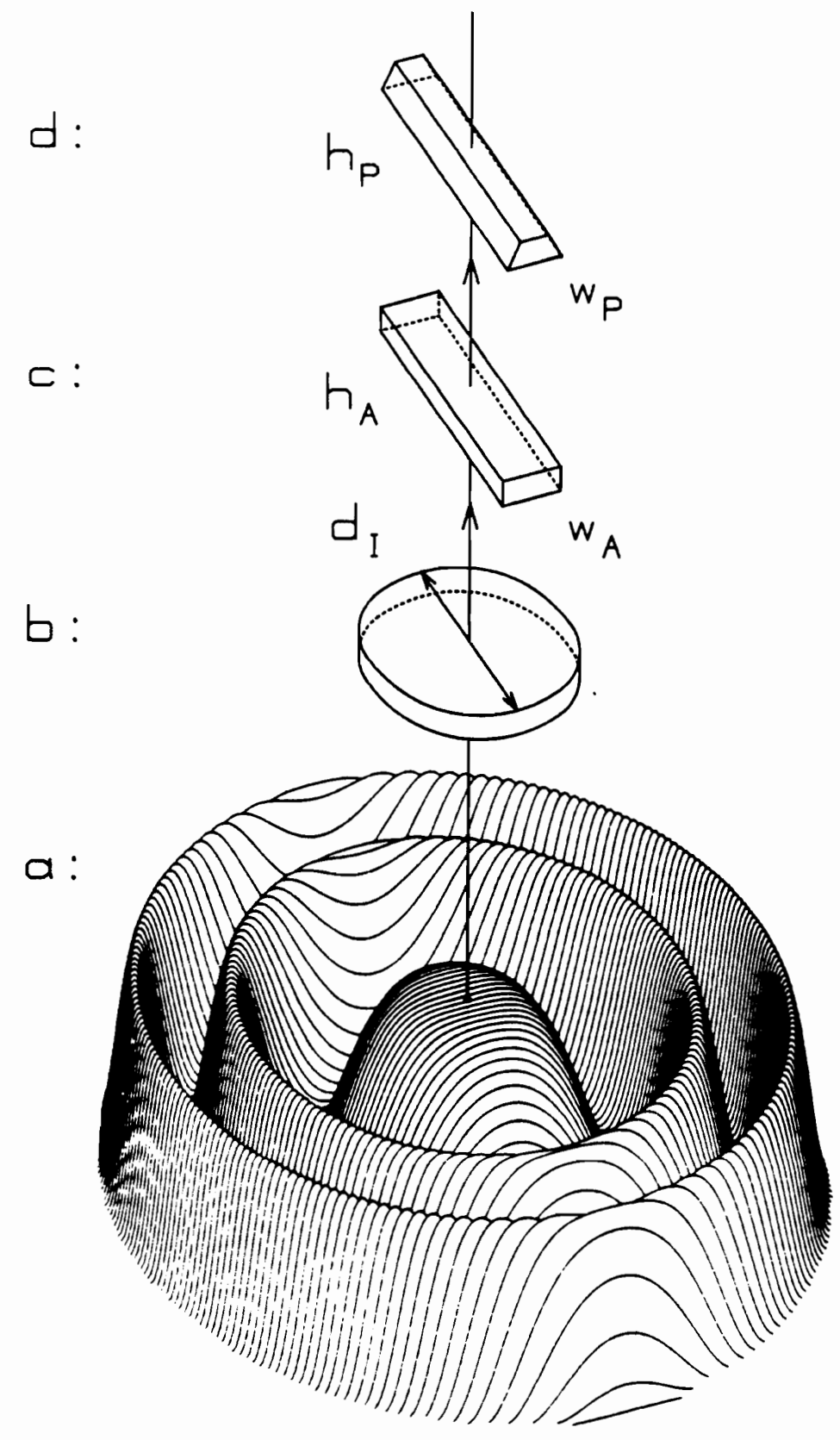

Figure 59. Haidinger fringes and interaction with the MMS apertures: This Figure shows the spatial response functions of a typical interferometer and the MMS, and their relationship to Haidinger fringes. All functions have a relative height of 1 . a) shows the intensity of the light at the output plane of an interferometer. b) shows the spatial response of a typical interferometer detector, where $d_{l}$ is the diameter of the detector aperture. c) shows the response of the MMS transfer aperture, where $h_{A}$ is the height of the aperture, and $w_{A}$ is the width of the aperture. d) shows the spatial response of the PDA pixel, where $h_{P}$ is the height of the pixel and $w_{P}$ is the width of the pixel. Adapted from reference 108. 
height of the pixel response function, $h_{P}$, is fixed. Therefore, the height of the transfer aperture, $h_{A}$ must be adjusted to conform to the requirements of Haidinger fringes. Consequently, the height of the transfer aperture usually limits the MMS throughput in the vertical plane.

The spectral discrimination capability of the MMS in either mode of operation can be predicted by convoluting (c) and (d) in the horizontal direction. In the dispersive mode, the convolution produces the instrument line shape, or the MMS response to a monochromatic line. In the interferometric mode, the convolution of the same two functions results in the spectral filtering function, shown in Figures 11 and 12.

\section{Complementary Fringes: Fizeau Fringes and Haidinger Fringes}

The Michelson interferometer produces two sets of complementary fringes, Haidinger and Fizeau fringes, shown in Figure 60. These sets of fringes were critical in the daily alignment of the MMS interferometer.

The sole purpose of the interferometer is to produce Haidinger fringes with the greatest possible contrast. These fringes are circular and result from equal inclination, or equal angle, through the interferometer. The fringes are observed at the focal plane of the focusing lens, and with the MMS they are projected onto the transfer aperture instead of the detector.

Fizeau fringes are fringes of equal thickness, or distance, through the interferometer. They indicate the amount of wedge between the images of the plane mirrors. Fizeau fringes are important because they give a direct measure of the alignment of the interferometer, and are thus directly tied to the contrast of the Haidinger fringes. Fizeau fringes can be observed at any plane past the beamsplitter. All regions within one fringe area represents a beam section with same path difference. Moving from one dark fringe area to another indicates a path difference between the areas of one wavelength. As an interferometer is brought into alignment, the number of fringes is reduced until the entire beam is composed of one fringe, either light or dark. If an interferometer 


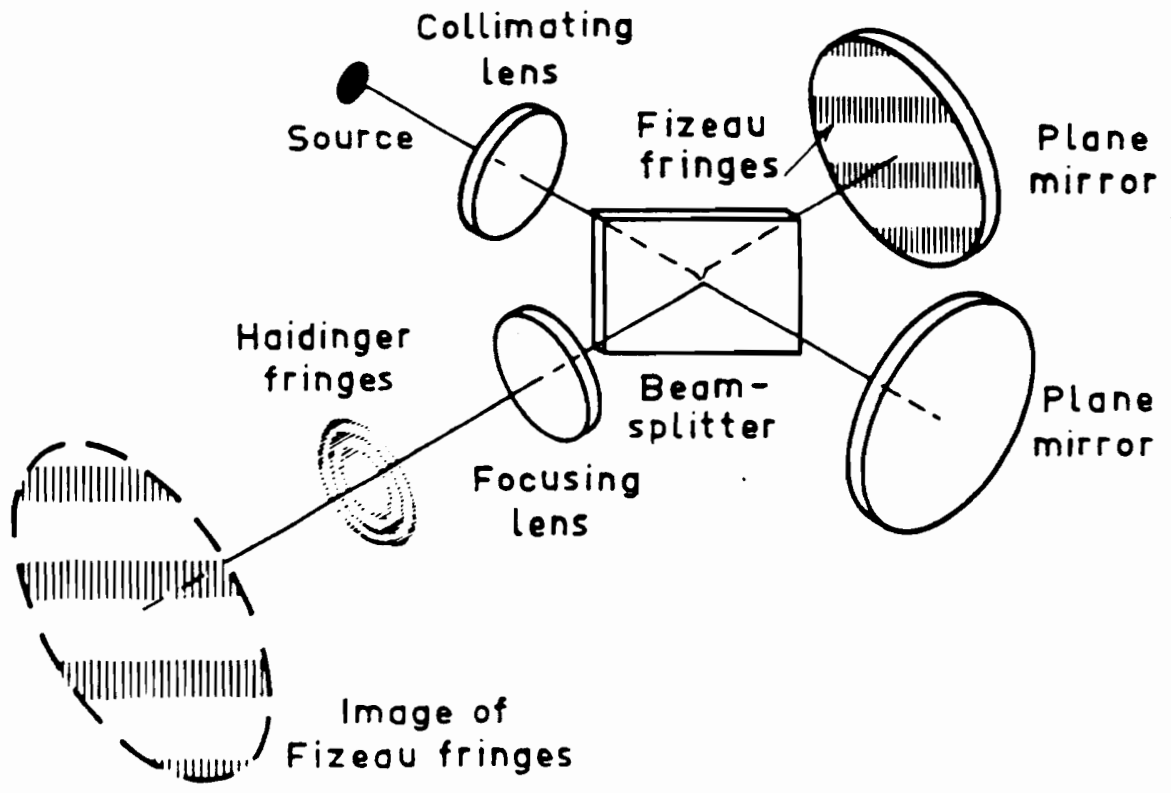

Figure 60. Haidinger and Fizeau fringes: Complementary fringes within the interferometer 
is out of alignment, the number of fringes represents the amount of misalignment, and a line perpendicular to the fringe pattern indicates the direction of misalignment.

With the MMS, these fringe sets were used with great success in the alignment procedure of the interferometer. Briefly, the procedure involved placing a monochromatic point source at the input, placing a screen in front of the focusing lens, and monitoring the number and direction of the Fizeau fringes while adjusting the fixed mirror alignment. This procedure allowed alignment to \pm 1 wavelength of path difference across the beam. Sub-wavelength alignment could be done by placing the screen at the focal plane at the transfer aperture and then visually maximizing the Haidinger fringe contrast. Haidinger fringes give neither degree or direction of misalignment however, so this last operation was much more subjective than the first.

\section{Apodization}

As was shown previously, the existence of a finite path difference causes ringing in the final spectrum. Apodization is a mathematical procedure used in FTS to control the line shape and to reduce ringing in the line wings. Typically, the interferogram is multiplied by an appropriate function. The apodization function tapers the interferogram to near zero at the ends. Multiplication of the interferogram by the apodization function is equivalent to scanning (convoluting) the spectrum with a weighted filter. Apodization is usually done if the resolution is too low and self-apodization has not tapered the interferogram sufficiently. There are a number of good discussions of apodization in the literature. ${ }^{109,110}$

Figures 61 and $62^{110}$ show a number of common apodization functions and their FT pairs. The left columns show the apodization functions. The right columns show the corresponding line shapes and their relative magnitudes. Listed with the line shapes are $\Delta \mu_{1 / 2}$, the full width at half maximum, and $S$, the amplitude of the first sidelobe as a percentage of the line maximum.

In the MMS, the boxcar (no apodization), the cos (Hamming) function, and the Gaussian function are user selectable for apodization. The cos is used because of easy implementation and 


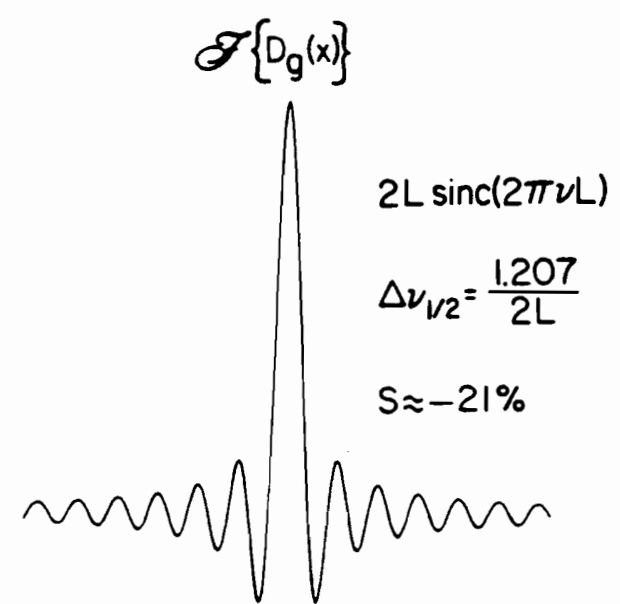

B. tropezoidal $\left\{2 D(x)\left[1-\frac{|x|}{L}\right]-D(2 x) \cdot\left[1-\frac{2|x|}{L}\right]\right.$

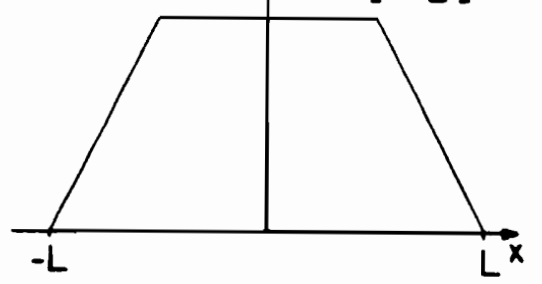

$2 L \operatorname{sinc}^{2}(\pi \nu L)-\frac{L}{2} \operatorname{sinc} c^{2}(\pi u / 2)$

$\Delta \nu_{1 / 2}=\frac{1.546}{2 L}$

$S \approx-15 \%$
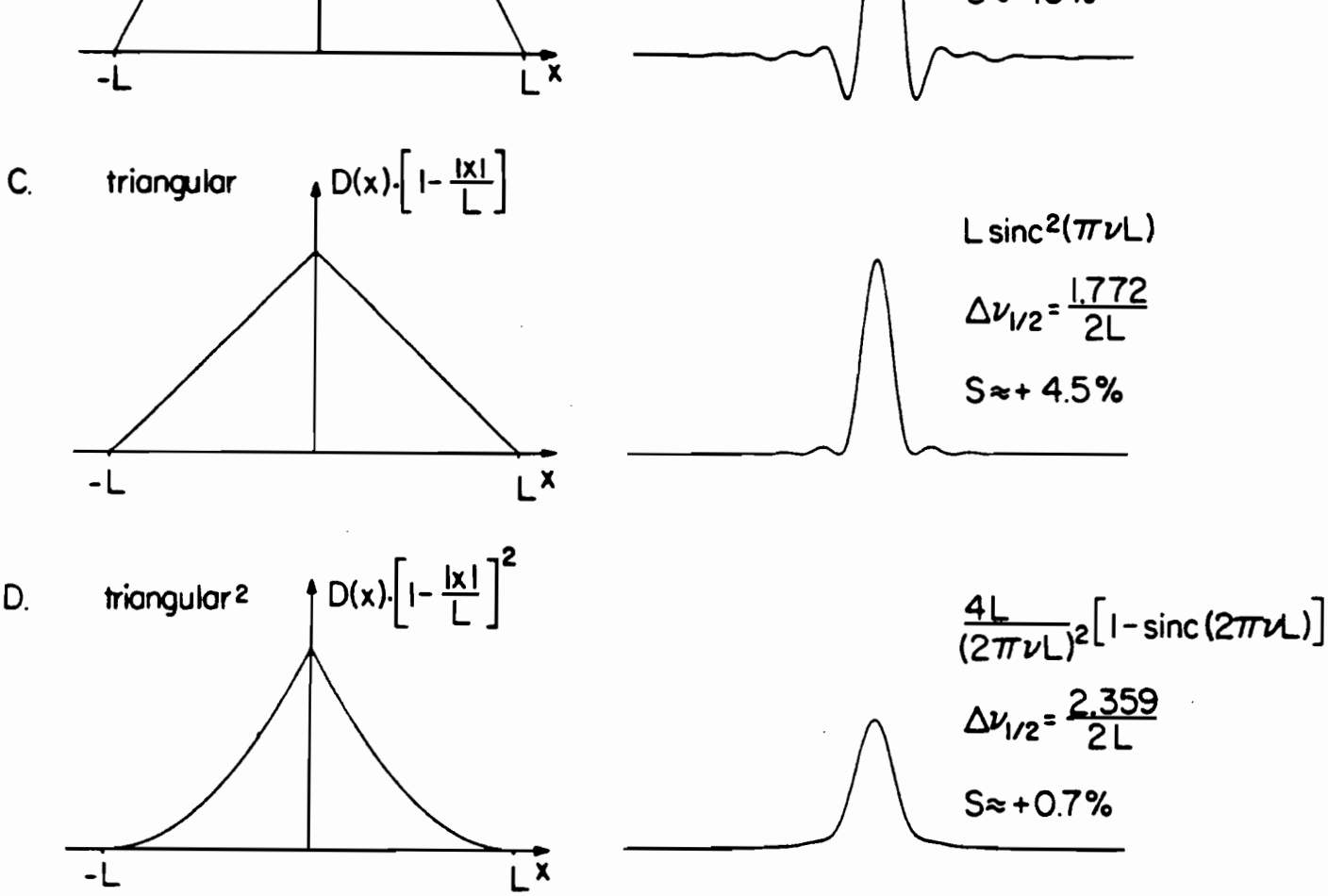

Figure 61. Common apodization functions used in FTS: Typical apodization functions and their FT pairs, the ILS functions, are shown. $\Delta v_{1 / 2}$ is the ILS width at FWHM, in terms of $L$ the maximum path difference. $S$ is the amplitude of the largest side lobe, given as a percentage of the function maximum. 


$$
D_{g}(x)
$$
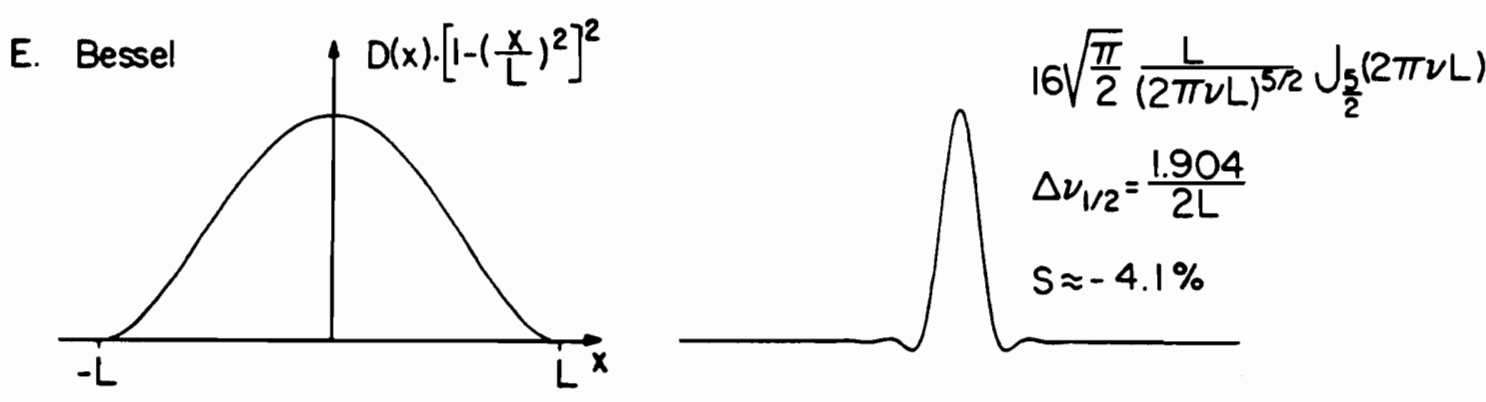

F. $\cos$
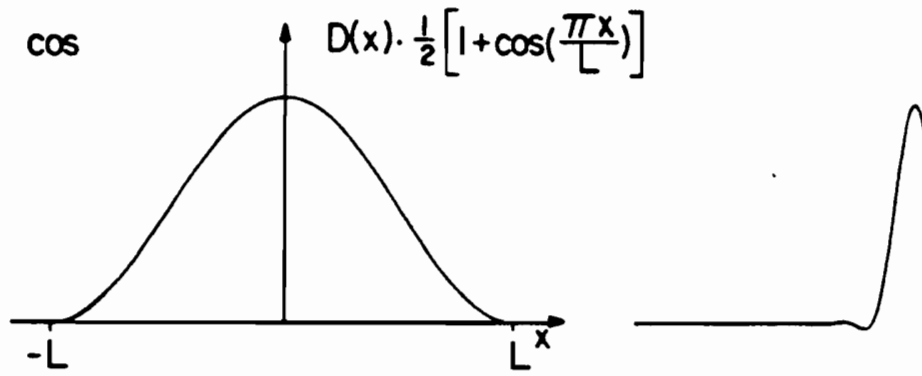

$\operatorname{sinc}(2 \pi \nu L)$

$\frac{\operatorname{sinc}(2 \pi \nu L)}{2 \pi \nu\left(1-4 L^{2} \nu^{2}\right)}$

$\Delta \nu_{1 / 2}=\frac{2.000}{2 L}$

$S \approx-2.7 \%$

G. $\operatorname{sinc}^{2}$
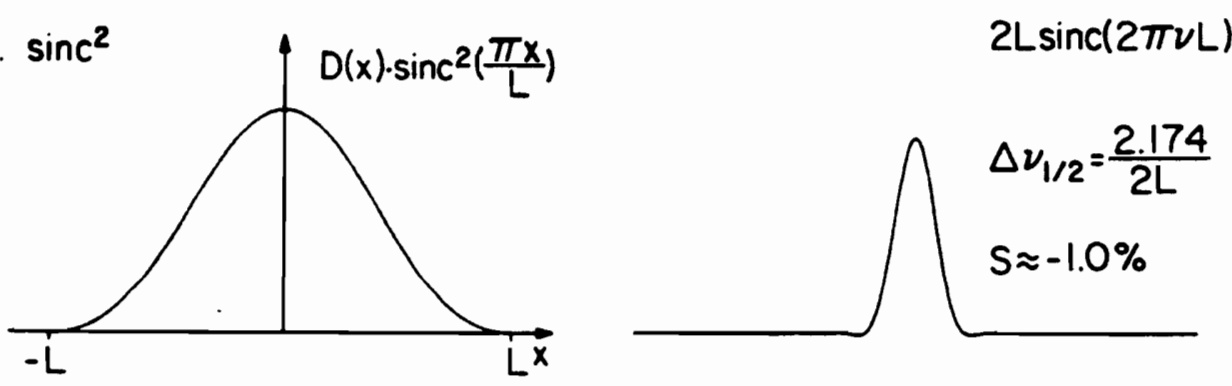

H. Goussion

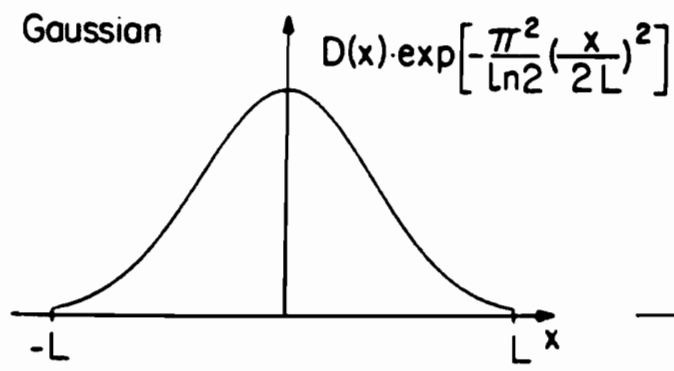

$\sim \frac{2 L \sqrt{\ln 2}}{\sqrt{\pi}} \exp \left[-(2 L L)^{2} \ln 2\right]$

$\Delta \nu_{1 / 2}=\frac{2.030}{2 L}$

$S \approx-0.45 \%$

Figure 62. Common apodization functions, continued: Typical apodization functions and their FT pairs, the ILS functions, are shown. $\Delta v_{1 / 2}$ is the ILS width at FWHM, in terms of $L$ the maximum path difference. $S$ is the amplitude of the largest side lobe, given as a percentage of the function maximum. 
a good performance. The Gaussian is used because of the generally Gaussian line shape found in AES sources ${ }^{30}$, reduced complexity when deconvoluting Gaussian lines,,$^{50}$ and good performance..$^{50}$

\section{Phase Correction}

Phase correction is motivated by concerns about noise in the final spectrum. As shown in Figure 53, errors in FTS caused the spectrum to twist out of the Real plane. These errors arise from the beam splitter, the analog electronic filters on the detector, and a sampled interferogram which is slightly offset and asymmetric from the actual interferogram. If noise were not a problem, Equation 17 could be used to find the signal amplitude at a given frequency. Noise, however, makes the problem more complex. Figure 53 will be used to explain the difficulties.

In FTS, white Gaussian-distributed noise in the interferogram comes across the Fourier transform as white Gaussian-distributed noise in both the Real and Imaginary planes, with an average of zero in both planes. ${ }^{50}$ In the three dimensional representation shown in Figure 53, the noise in the spectrum has the appearance of an old, worn test-tube brush placed along the frequency axis. The bristles of the brush correspond to the noise vectors.

If the spectrum can be phase-corrected, the spectral vectors are rotated back into the Real plane. Three results come out of this. One, the noise components orthogonal to the spectral vectors can be ignored. Two, the noise distribution in the spectrum remains Gaussian, with an average of zero. Three, because of the Gaussian baseline noise, spectra can be added to increase $\mathrm{S} / \mathrm{N}$.

If the spectrum cannot be phase-corrected, Equation 17 must be used, and there are three corresponding results. One, all noise vectors are rectified and are brought into the spectral plane. Two, the noise distribution becomes a Rayleigh distribution, with a non-zero average. Three, because of the rectified noise, noise between spectra adds in a nonlinear fashion, and spectra cannot be added to improve the $\mathrm{S} / \mathrm{N}$. 
Phase correction in FT-AES is very difficult. Typical phase correction algorithms for the IR assume that there is enough spectral information at enough wavenumbers to determine the phase error function as it wanders along the phase cylinder in Figure 53. In AES, however, the spectrum is usually very sparse, with regions having no spectral energy, and therefore yielding no information on the phase errors. Several papers have been published which describe a phase correction algorithm for FT emission spectrometry. ${ }^{111,112}$ This method uses a weighted, seventh-order polynomial to extrapolate phase information from regions of high spectral intensity into region of low spectral intensity.

Phase correction is even more complex with the MMS, and therefore was not preformed. The specific difficulties include the following. The presence of the PDA in the MMS system causes two unique phase errors. These are due to the integrating nature of the PDA, and its sequential readout scheme. See the photodiode array chapter. In addition, the spectral information needed to perform phase correction is even more sparse in the MMS because of the spectral filtering by the PDA and the polychromator. Also, to arrive at a global phase error function for the entire array and spectrometer, phase information would have to be exchanged between interferograms acquired by the different pixels. Finally, advanced phase correction algorithms require much larger computers than those in the MMS system.

\section{Mirror Scanning: One-sided vs. Two-sided Interferograms}

The most common mirror-scanning technique used to record interferograms involves starting the mirror near zero path difference and observing only one half of the interferogram. The reason for these one-sided interferograms is that the ideal interferogram is symmetric. There are several advantages to assuming symmetry: an interferometer can attain twice the resolution for a given mechanical scan limit, the data can be acquired in one-half the time, and only one-half the data points need to be recorded. With this technique, a short portion of the symmetrical 
interferogram is recorded to allow phase correction, but the asymmetry portion is typically $95 \%$ of the interferogram. Most FT-IR spectrometers use the single-sided recording.

In FT-AES, however, there are a number of compelling reasons to record two-sided interferograms. First, when no phase correction is possible, two-sided interferograms must be taken to prevent line distortion. ${ }^{113,114}$ Second, if phase correction is possible, using two-sided interferograms causes the remaining phase errors to have a much lower effect on the line shape. ${ }^{112}$ This point is very important in high resolution FT-AES, which is often motived by interest in line shape, and where phase correction is much more difficult.

Two-sided interferograms were acquired with the MMS because of the inability to do phase correction in the present MMS system. 


\section{Photodiode Array Theory}

\section{Introduction}

This section discusses the performance and physical characteristics of the photodiode array detector, particularly as they pertain to the performance of the MMS. The spectral, spatial, and temporal response of the PDA are presented. The noise sources within the PDA are discussed. The PDA noises are particularly significant, because the PDA adds several types of noise to the MMS output, and because detector read noise proved to be the limiting noise in the MMS.

As the detector of the MMS, the PDA obviously affects the spectral response and spectral range of the MMS in all modes of operation. The PDA also plays primary roles in spatial and temporal signal processing within the MMS. The spatial response function of the individual PDA diodes is central to spectral discrimination in all modes of operation.

The PDA is an integrating detector, and as such it has a specific temporal frequency response, or transfer function. Understanding the temporal response of the PDA is important in a number of areas of the MMS design. Due to its frequency response, the PDA attenuates higher frequencies and also causes a phase shift of higher frequencies. Proper coordination between scanning the interferometer mirror and reading the detector array was therefore critical in matching 
the range of frequencies produced by the mirror velocity to the frequency response range of the PDA. In addition, the transfer function is carefully utilized to enable the PDA to be the primary temporal frequency filter in the MMS. By tuning the PDA's temporal response, it provides noise rejection, $\mathrm{AC}$ detection in the interferometric mode, and $\mathrm{DC}$ detection in the dispersive mode.

\section{Criteria for a Solid State Detection System}

The detector system for the MMS was selected from the three basic solid-state technologies available for multichannel detectors: photodiode arrays (PDAs), charge coupled devices (CCDs), and charge injection devices (CIDs). The decision was based upon the following performance criteria: ${ }^{7,5,115,9,116}$

- Rapid readout rate.

- High quantum efficiency for high sensitivity.

- Linear response, signal charge vs. exposure.

- A wide spectral range, with response over the entire range of interest in AES.

- Large full-well capacity.

- Low read noise.

- Large dynamic range: [ $\left.\frac{\text { Full well capacity }}{\text { Read noise }}\right]$

- Resistance to blooming at full-well capacity.

- Low dark current to prevent saturation of the array.

- Low readout lag.

- Large active area.

- Large pixel count.

- Geometric accuracy.

- Stability. 
- Reliability.

- Reasonable cost.

The detector selected for the MMS is the RL-0512S photodiode array from Reticon Corporation. PDA technology meets most of the criteria very well. The RL-0512S was chosen particularly for its wide spectral range, high quantum efficiency, linear response, rapid readout rate, and large dynamic range. In addition, the integrated readout circuitry, the simple readout control, and the availability of an easily modifiable PDA controller board lent this array to a spectrometer evaluation project. One drawback of PDAs is their large read noise relative to other solid-state detectors. At the inception of this project, PDAs were the most appropriate detector technology. Solid-state detector technology continues to evolve rapidly, however, warranting reevaluation of the detection system for future improvements to the MMS. CCDs are particularly attractive because of low read noise.

\section{Description of Photodiode Array and the Snatial Response}

\section{Function}

The RL-0512S is a monolithic self-scanning linear photodiode array with silicon sensor elements. The array has been optimized for use in spectroscopy. ${ }^{17}$ The individual pixels are 2.5 $\mathrm{mm} \times 25 \mathrm{um}$ and have a 100:1 aspect ratio, providing a large sensor area and a shape approximating the slit size of a typical dispersive spectrometer. The technical specifications for the RL-0512S are listed in Table 26. The RL-0512S chip selected for this work is fitted with a quartz window for greater spectral response in the UV. ${ }^{17}$

The PDA sensor structure within the silicon wafer is shown in Figure 63A and B. As shown in Figure 63B, the individual diodes are formed by diffusing p-type dopants into an n-type 
Table 26. Photodiode Array Specifícations.

Array Detector:

Type:

Model:

Manufacturer:

Photodiode array, 512 element

self-scanning, linear

RL-0512S

Reticon Corp., Sunnyvale, CA

PDA Specifications:

Pixel number:

Pixel size:

Total photoactive area:

Readout rate:

Quantum efficiency:

Linearity:

Spectral range:

Dynamic range:

Saturation charge:

Full well capacity:

Read noise:

Dark Current:

'25'primemark degree $C^{b}$

Blooming:

Readout lag:

Fixed pattern:

Geometric accuracy and precision:

Reliability.:

Capacitance of pixel:

Capacitance of each video line:

Capacitance of each clock line:

$512^{\circ}$

$2.5 \mathrm{~mm}(\mathrm{~h}) \times 25 \mu \mathrm{m}(\mathrm{w}) 。$

$62,500 \mu \mathrm{m}^{2}$ 。

$2.5 \mathrm{~mm}(\mathrm{~h}) \times 12.8 \mathrm{~mm}(\mathrm{w}){ }^{a}$

$32.0 \mathrm{~mm}^{2}$ a

$5 \mathrm{MHz}, \max$.

$80 \%$ at $650 \mathrm{~nm}^{\circ}$

Excellent over full range ${ }^{\circ}$

$200-900 \mathrm{~nm}^{\circ}$

at $>40 \%$ QE a

$>10,000: 1$ 。

14 pcoul $\circ$

$8.7 \times 10^{7} \mathrm{e}^{-。}$

$>1,200 \mathrm{e}^{-b}$

5 pamp at

$<1 \%$ b

$<0.1 \%$ b

$1 \%$ of full scale ${ }^{b}$

High $b$

High $^{b}$

$2 \mathrm{pF}^{a}$

$12 \mathrm{pF}^{a}$

$30 \mathrm{pF}^{a}$

a. From reference 17, pp 1-33 to 1-36.

b. From reference 7 . 
silicon substrate. The spatial response function of the individual diodes is shown in Figure 63C. The function results from the probability of photoelectrons to diffuse to a given depletion region under a p-type bar.

The equivalent circuit of the internal PDA components is shown in Figure 64. Integrated into the same monolithic device are the active diodes (pixels), the digital shift registers and MOS transistors for self-scanning readout, and the dummy diodes for analog subtraction of switching noise and fixed pattern signal from the active diode signal.

A simulated PDA output waveform for an AES spectrum is shown in Figure 65 . The important feature to note is the rectangular output pulse for each pixel. The rapid transitions between pixels require very fast wide-band amplifiers to follow the spectral changes with fidelity. In the MMS, amplifiers with $20 \mathrm{MHz}$ bandwidths and $600 \mathrm{~V} / \mu \mathrm{sec}$ slew rates were used to accommodate the $2 \mathrm{MHz}$ pixel output rate over a $\pm 5 \mathrm{~V}$ output range. The transitions also prohibit the use of analog filtering to reduce noise in the output. The PDA temporal response was utilized as a substitute.

\section{The Spatial Response Function}

The shape of the PDA spatial response function, shown in Figure 63C, has considerable effect on optical throughput and spectral discrimination capabilities of the MMS. It essentially defines the width and response across the MMS output slit. Together with the pixel height it defines the shape and response of the MMS exit slit. As discussed in Chapter 3, the spectral discrimination capability of the MMS in any mode of operation can be predicted by the convolution of the PDA spatial response function with the transfer aperture in the horizontal plane. In the interferometric mode, the shape of the spatial response function is central to spectral filtering. In the dispersive mode, the individual diodes act as the exit slits for the polychromator. Consequently, spatial response is a fundamental determinant of spectral resolution. 
a)

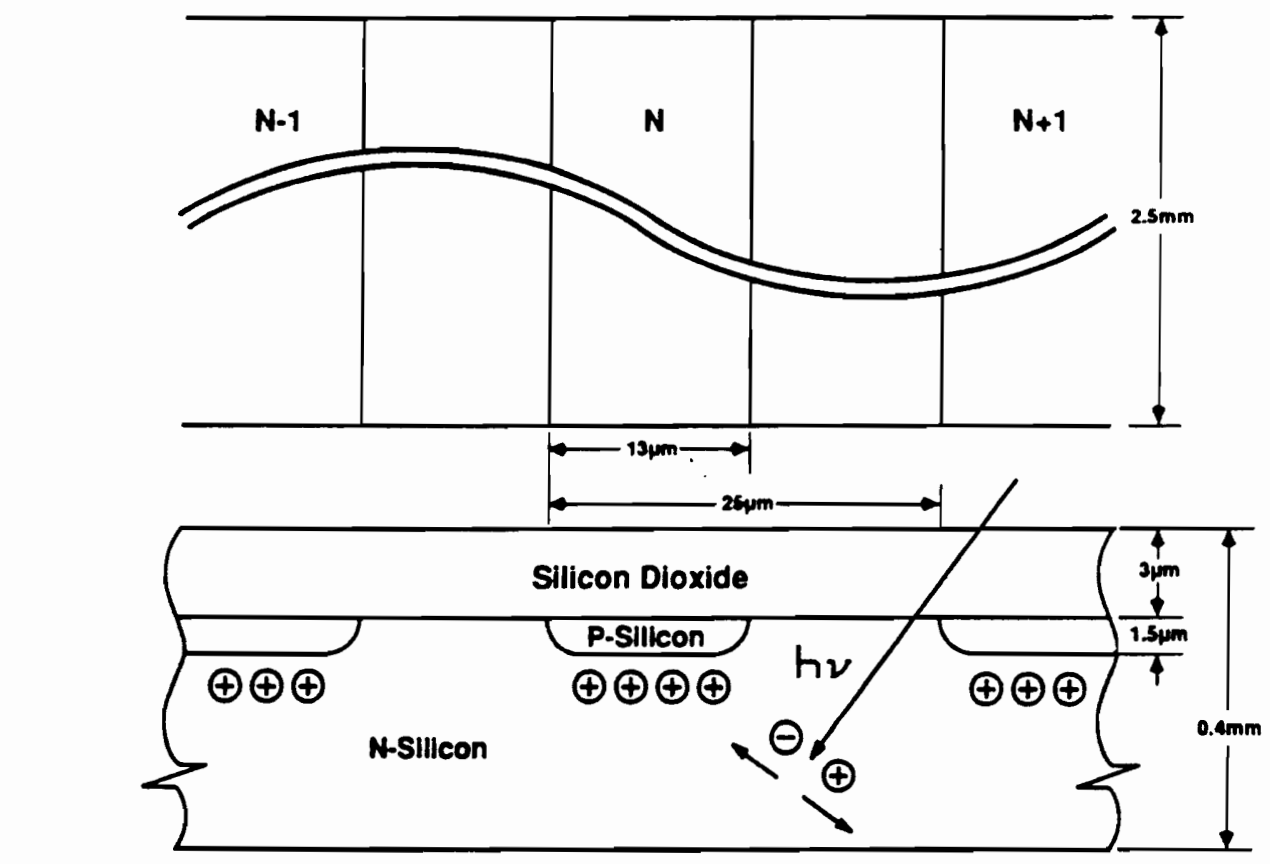

b)

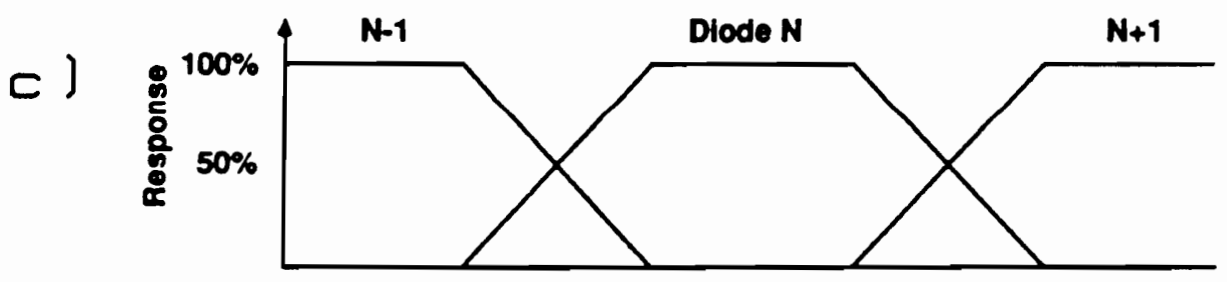

Position of Incident Light Spot

Figure 63. The PDA sensor geometry and the spatial response function: a) Front surface geometry of the pixels. b) Cross-sectional geometry. c) Spatial response function resulting from the diode structure. Adapted from reference 17. 


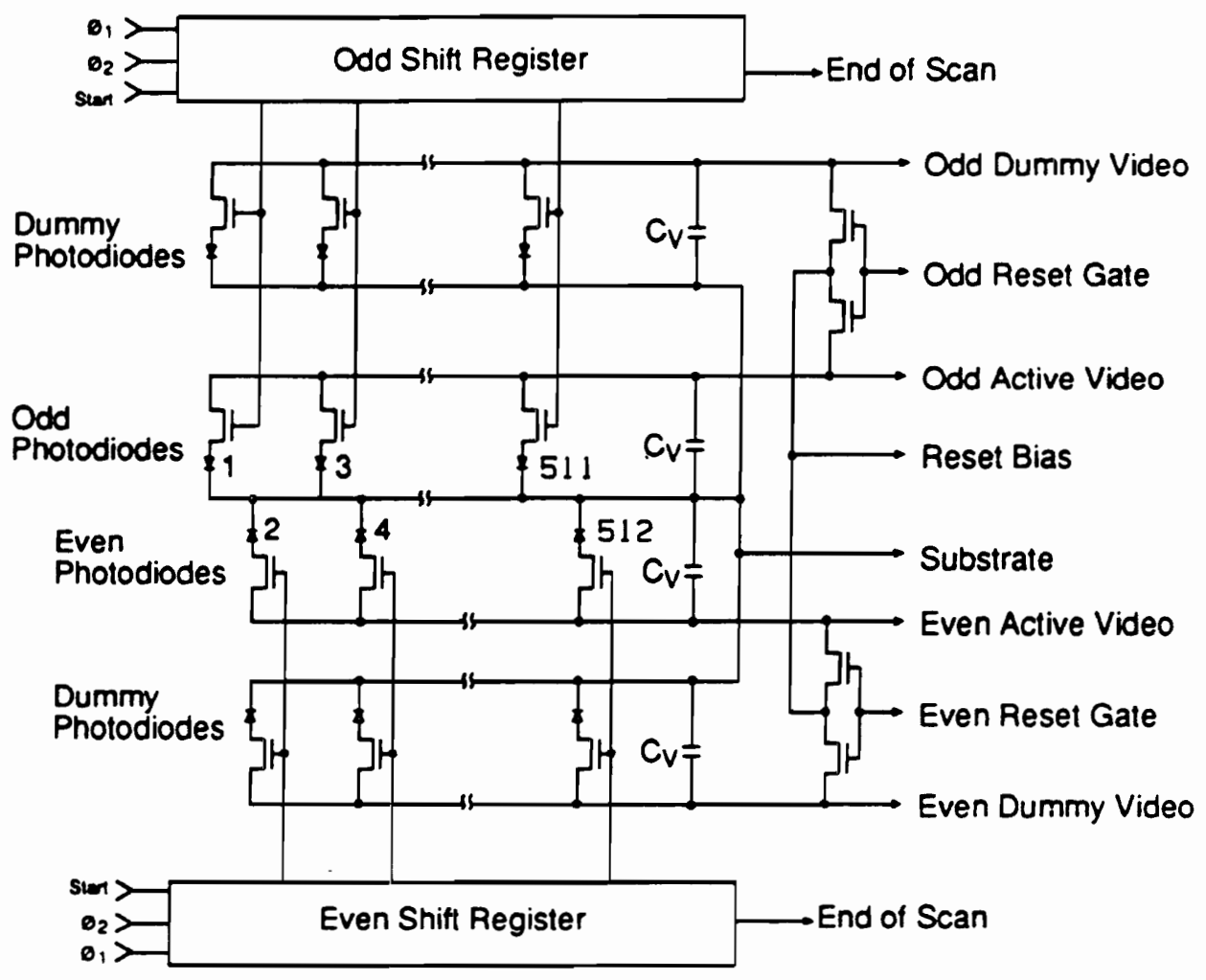

Figure 64. The equivalent circuit of the RL-0512S photodiode array: The active photodiodes, digital shift registers, MOS transistors, reference "dummy" diodes, photodiodes, control lines, and output lines are shown. Adapted from reference 17. 


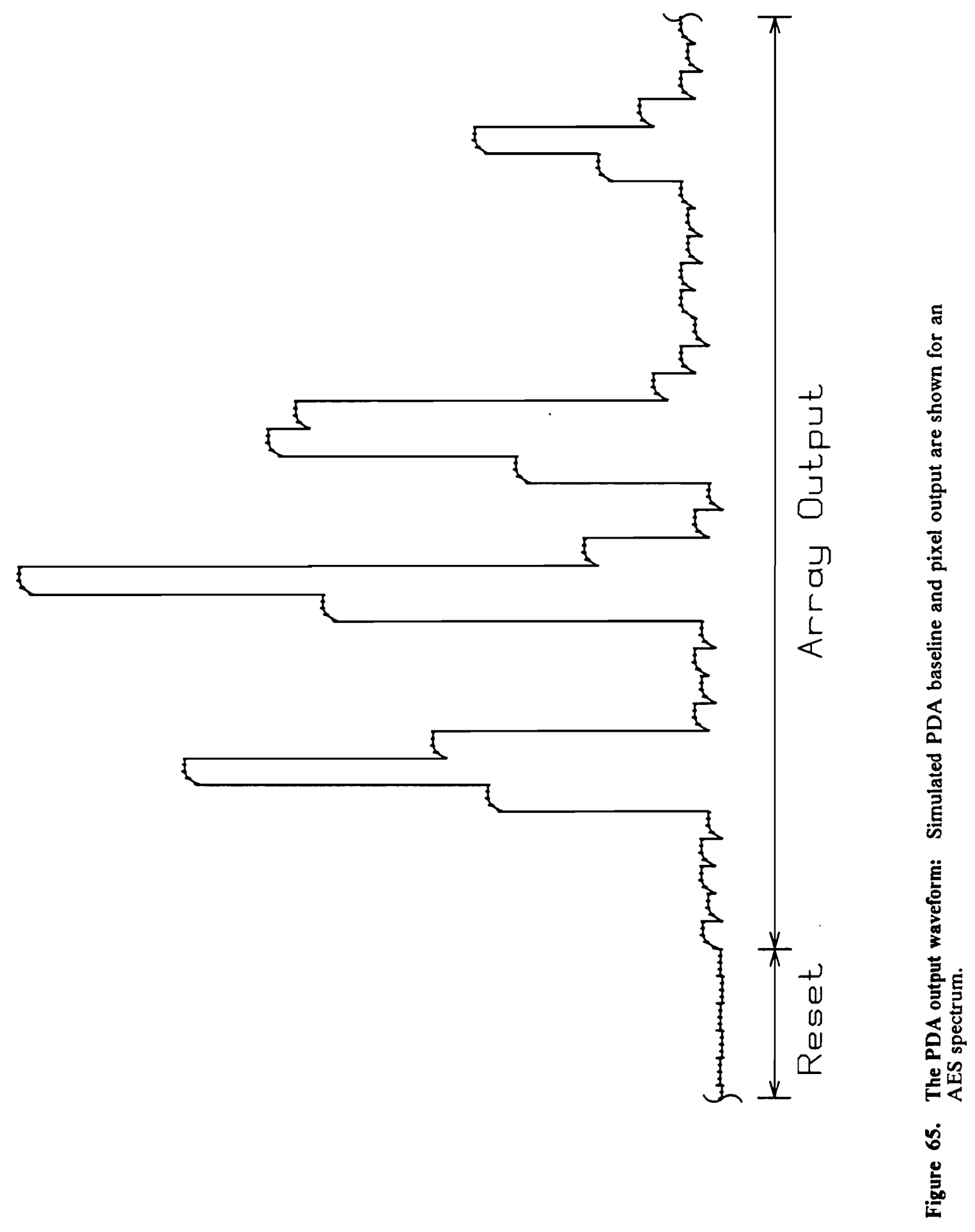




\section{The Spectral Response Function}

The PDA spectral response functions for an S-series array with a quartz window are shown in Figure 66.17 Quantum efficiencies (QE) are shown, rather than radiometric responsivities, because response to discrete events is more appropriate for AES than spectral energy per nanometer. These Figures represent attenuations factors for the incident photon beam. Figure 66A is the attenuation factor directly applicable to dispersive mode spectra. Figure $66 \mathrm{~B}$ is the attenuation factor applicable to interferometric mode spectra. The horizontal axis in Figure 66B is also labeled with the optical modulation frequencies produced by the interferometer, recognizing the frequency/wavenumber duality in the interferometric mode.

As shown in the Figure 66, the quantum efficiency of the PDA is quite high across the UV and visible, with a peak QE of $80 \%$. Over the AES range of interest, the QE ranges between approximately $40 \%$ to $75 \%$. This is in contrast to a typical photomultiplier tube peak QE of $20 \%$ to $25 \%$. A high quantum efficiency is important to an improved $S / N$ in the detected signal when the effects of the photon shot noise of the incident photon beam are considered. ${ }^{118}$ This is given by

$$
S / N_{d}=\sqrt{K_{\lambda}} S / N_{\phi}
$$

where $S / N_{\phi}$ is the $S / N$ in the photon beam due to photon shot noise, $K_{1}$ is the detector quantum efficiency, and $S / N_{d}$ is the resulting $S / N$ in the detected signal. 
A

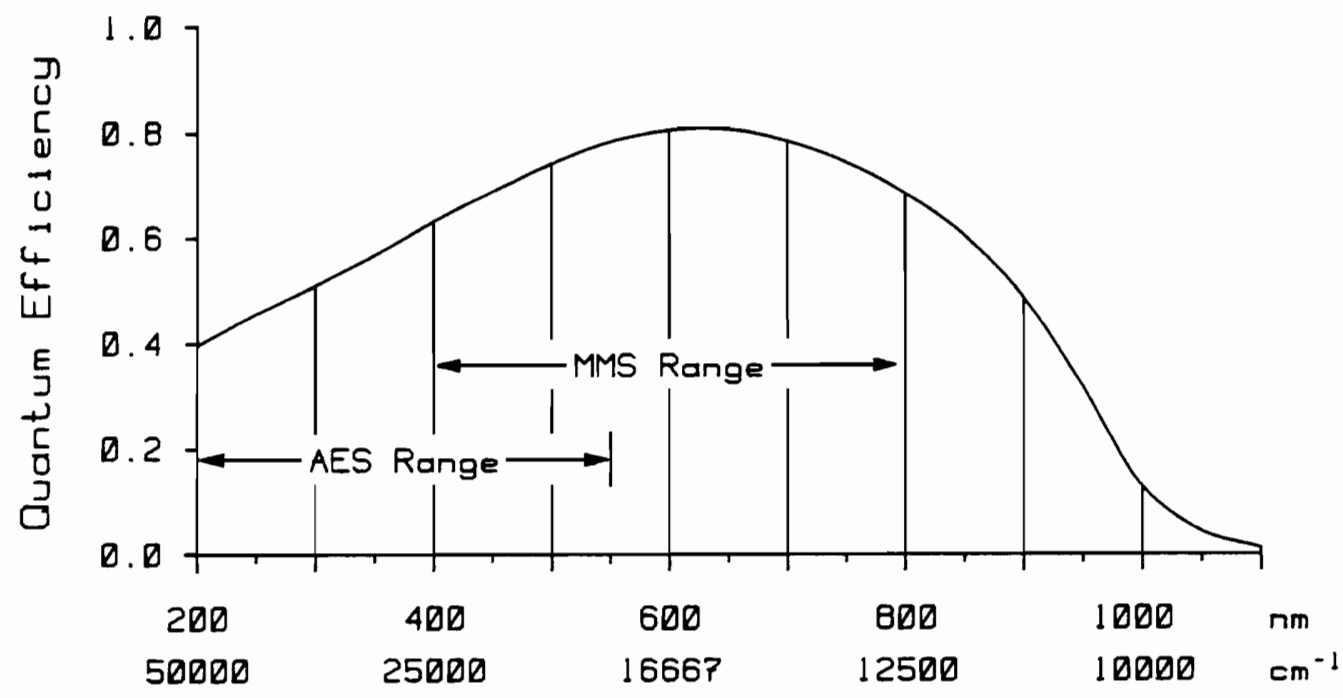

B

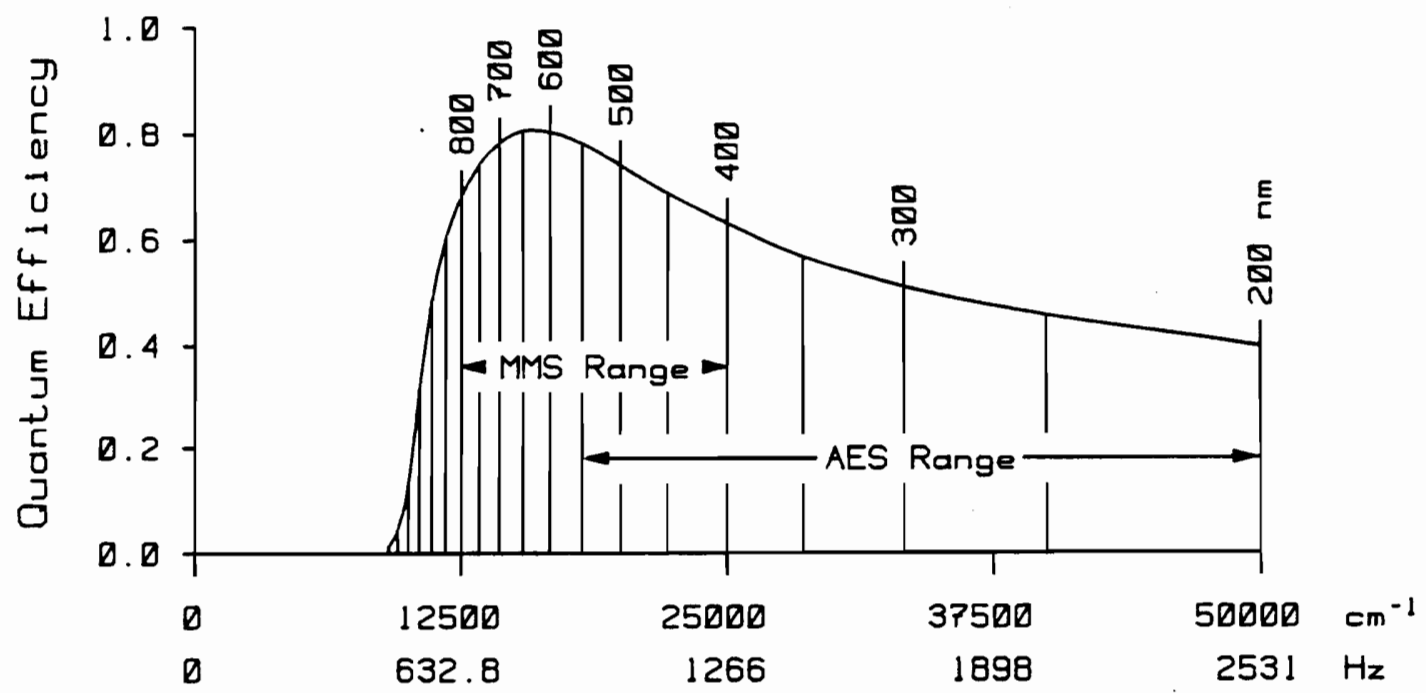

Figure 66. The spectral response and quantum efficiency of the S-series photodiode array: Figure $A$ shows QE vs. wavelength. Figure B shows QE vs. wavenumber. The horizontal axes for Figure $B$ is labeled with the corresponding optical modulation frequencies for interferometric spectra. Vertical bars in both Figures are at $50 \mathrm{~nm}$ intervals. Adapted from reference 117 


\section{The Temporal Response Function}

\section{Introduction}

The ability of the PDA to respond to certain time-varying signals and its ability to suppress others were very critical issues in the development of the MMS. Understanding the PDA response was also important for the proper interpretation of the amplitude and phase data produced in the interferometric mode. As an integrating detector, the PDA can severely attenuate various frequencies, and can cause a related phase shift in the detected signal. In particular, if the PDA integration time is exactly equal to an integer number of wave periods, the wave is completely attenuated. As a rule of thumb, the integration time must be kept short relative to the wave period, less then $10 \%$, to avoid attenuation. In the interferometric mode, a fundamental requirement is the detection of the interferometer modulations. Hence, the PDA must be scanned rapidly relative to the modulations of the sample beam. However, the PDA cannot be scanned infinitely fast nor the interferometer infinitely slowly. Some reasonable compromise between the scan speed of the interferometer and the scan rate of the PDA had to be found. The associated amplitude attenuations and phase shifts needed to be analyzed to choose a reasonable compromise.

In a parallel fashion, the dispersive mode requires interferometer modulations to be suppressed. Interferometer scan speed and PDA scan rate can be coordinated to produce the required suppression, but adequate prediction of the attenuation is required.

The purpose of this section, therefore, is to examine the fundamental frequency response of the photodiode array. Specifically, the amplitude and phase response are discussed. The effects of the response on signal and on noise, the effects under aliasing, and the ability to use these effects to an advantage are examined. The response of the PDA to three types of photometric input was sought: One, the PDA response to DC light levels, primarily for the dispersive mode. Two, the PDA frequency response to the correlated modulations of the interferometer, for all modes of 
operation. Three, the PDA response to uncorrelated fluctuations due to photon shot noise, to predict the susceptibility of the MMS to aliased photon noise.

It should be emphasized that this section applies to other integrating array detectors such as CCDs or CIDs, as well as PDAs. It is also applicable to dual slope integrating $\mathrm{A} / \mathrm{Ds}$ and to box-car digital filters. A spatial analog of this model is also applicable to spatial interferometers and the array detectors used in them. ${ }^{119,92}$

\section{The PDA Transfer Function: The Derivation}

The transfer function of the PDA sampling function is fairly simple to derive through use of Fourier transform theory. ${ }^{105,61}$ Figure 67A shows the PDA time sampling function, $\Pi\left(t_{P D A}\right.$, with a width of $t_{i}$. During data acquisition, $\Pi(t)_{P D A}$ is scanned past the input signal, $s(t)$. The scanning operation convolutes $\Pi(t)_{P D A}$ with $s(t)$. According to the convolution theorem then, this is equivalent to multiplying (attenuating) $S(f)$ by the Fourier transform of $\Pi(t)_{P D A}$.

The complex Fourier transform of $\Pi(t)_{P D A}$, the complex transfer function, is shown in Figure 67B and C. A transfer function is typically complex, and the amplitude and phase representation is usually more useful than the real and imaginary representation. Because the shape of the PDA sampling function is a $\Pi(t)$ function, the general shape of the amplitude portion is a $\sin c(f)$. function. The $\left|\sin c(f)_{P D A}\right|$ representation shown in Figure 67B eliminates the sign of the negative side lobes of $\sin c(f)_{P D A}$ and emphasizes the magnitude of the transferred signal. Figure 67C shows a linear phase shift with discontinuities at the $\pm \pi$ points in the vertical scale.

\section{The Amplitude Response}

Figure 67 illustrates a number of important points. First, the width of the transfer function depends only on $t_{t}$, and is independent of the sampling frequency and the Nyquist frequency. 

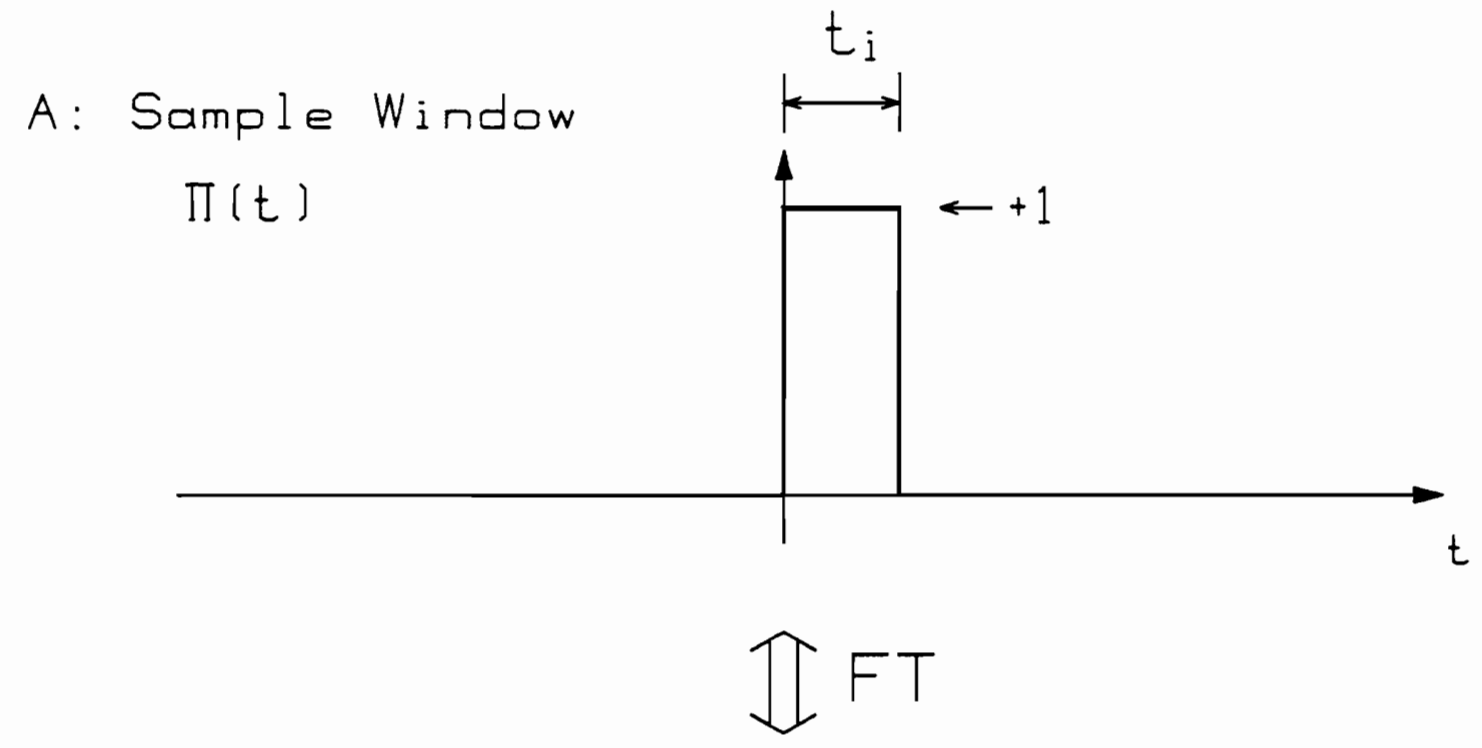

$B: A m p(f)$

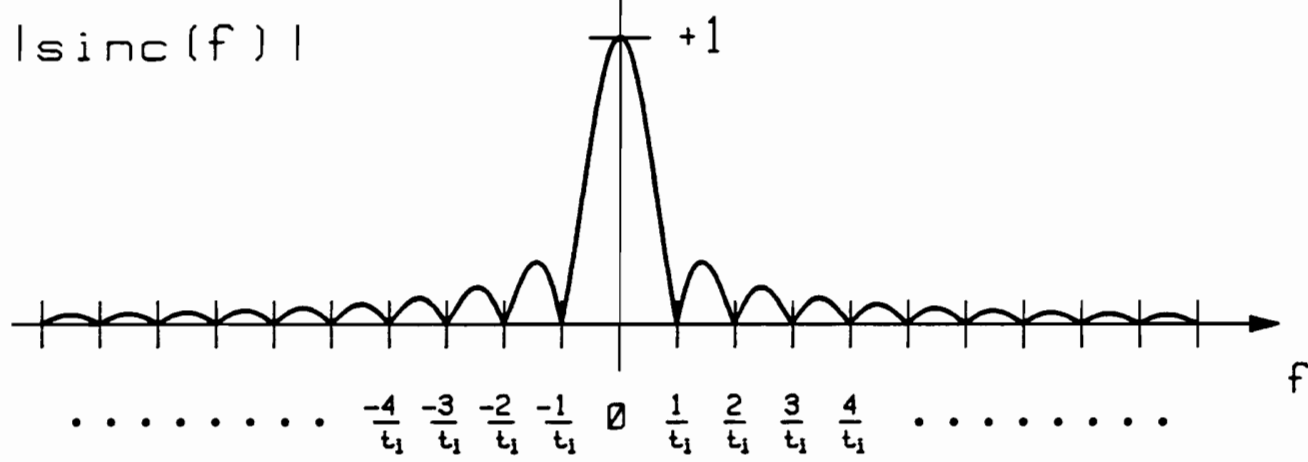

C: Phalf)

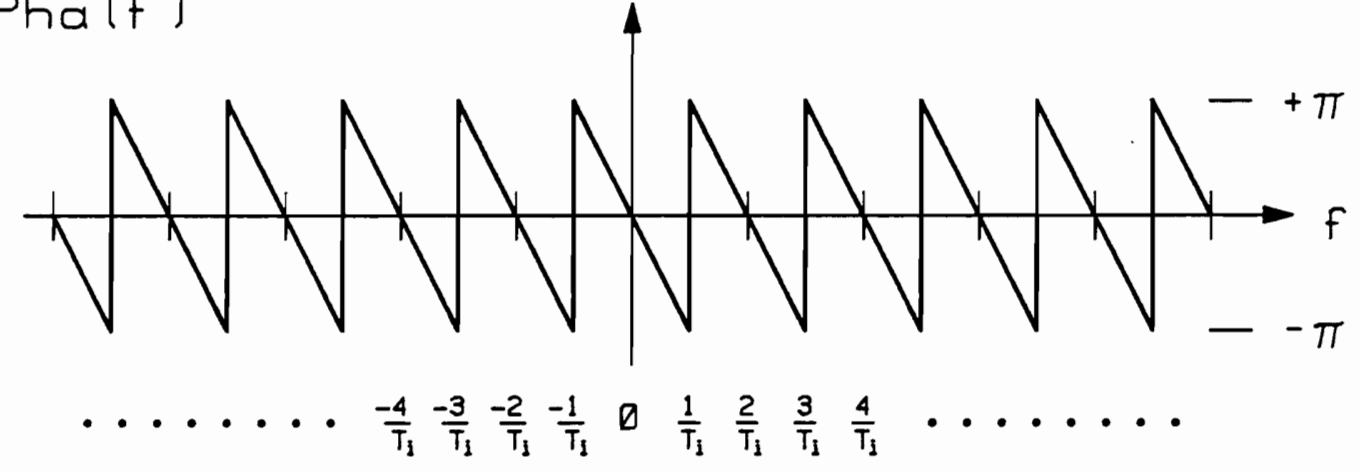

Figure 67. The PDA time sampling window and the normalized frequency response: a) shows a single PDA sample pulse time-shifted from the origin. b) and c) show the Fourier transform of the sample pulse in the amplitude/phase representation. 
Sampling frequency effects in the MMS interferometric mode, such as aliasing and the sample-limited bandwidth, must be considered separately. Second, only frequencies much less than $1 / t_{i}$ are unattenuated. Third, frequencies equal to $n / t_{i}$, where $n$ is an integer $\geq 1$, are attenuated completely. Fourth, if a higher frequency response to $S(f)$ is required, $t_{i}$ must be shorter. Reducing $t_{i}$ makes $\left|\sin c(f)_{P D A}\right|$ and the phase spectrum wider.

\section{The Phase Response}

Next, the origin and effects of the phase spectrum, $P h a(f)_{P D A}$, are considered. The phase shift results from the time delay of the integration window. In Figure $67 \mathrm{~A}, \Pi(t)_{P D A}$ is shifted on the time axis so that it begins at $(t=0)$ and ends at $\left(t=t_{i}\right)$, rather than being symmetrically displaced about the time origin. From the FT time-shift theorem, the resulting phase shift is linear and is given by

$$
\phi_{i}=-\frac{\pi t_{(i)}}{t_{x}}=-\pi t_{(i)} f_{x}
$$

where $\phi_{i}$ is the phase shift due to integration, $t_{i}$ is the integration time, and $t_{x}$ and $f_{i}$ are the period and frequency of the input wave respectively.

The shape of the phase curve means that input frequencies will experience a phase delay directly proportional to their frequencies. In most detection schemes, the phase shift is normally not significant, causing a slight skewing of the frequencies that compose a rapidly rising or falling signal edge, and producing a slightly rounded waveform. In an interferometric system, the phase shift may become important, and should be incorporated into the phase correction scheme. 


\section{Predicting PDA Frequency Response to General Inputs}

Several different formats of the PDA frequency response function are shown Figure 68. The different formats are useful for analyzing the temporal response of the PDA in different circumstances and to different inputs. The horizontal axes in Figure 68 are in normalized frequency units, $\bar{f}$, where $\bar{f}=f_{x} / t_{i}$, and $f_{x}$ is the absolute frequency of the input waveform. The vertical axes in Figure 68 are also normalized, thus representing attenuation values.

In this section, the effects of aliasing must be discussed. To connect the effects of integration time and sampling rate, it is assumed the array is read as fast as possible. In this case, the sampling frequency, $f_{S}$, is $1 / t_{i}$, and the Nyquist frequency, $f_{N}$, is $1 / 2 t_{i}$. Consequently, the base bandwidth is $f=0$ to $1 / 2 t_{i}$, and the folding points for aliasing occur at $\bar{f}=n / 2 t_{i}$ normalized frequency units, where $n$ is an integer.

Figure 68A shows the positive half of the sinc function. The sinc is the basis of the other Figures, and is included for reference. Figure 68B, the $\mid$ sinc $\mid$ function, shows the amplitude response of the PDA. $\mid$ sinc| is useful for predicting the PDA response to correlated modulations (nonrandom signals) in the base band, or modulations which may be aliased into the base band from higher bandwidths.

Figure 68C, $\sin ^{2}$, shows the power response of the PDA. $\operatorname{sinc}^{2}$ is useful for predicting the aliasing of uncorrelated modulations (random signals) from all frequencies into the base bandwidth. Photon shot noise, a wide-band white noise, is the noise of interest. Without proper filtering, the photon noise will be aliased back into the base band. Using Figure $68 \mathrm{C}$, the total susceptibly of the PDA to photon shot noise can be predicted by folding $\sin c^{2}$ at the alias points, and adding the overlapping portions of the $\operatorname{sinc}^{2}$ curve. As can be seen from Figure $68 \mathrm{C}$, $\operatorname{sinc}^{2}$ predicts a rapid attenuation of the photon noise frequencies above $1 / 2 t_{i}$. Consequently, the PDA acts as an effective low-pass anti-aliasing filter against high frequency photon noise.

Figure $68 \mathrm{D}$ shows the sinc function replotted in a log-log format, a format used in filter analysis. The top dashed line indicates the worst-case performance envelope. The $-3 \mathrm{db}$ point (the 

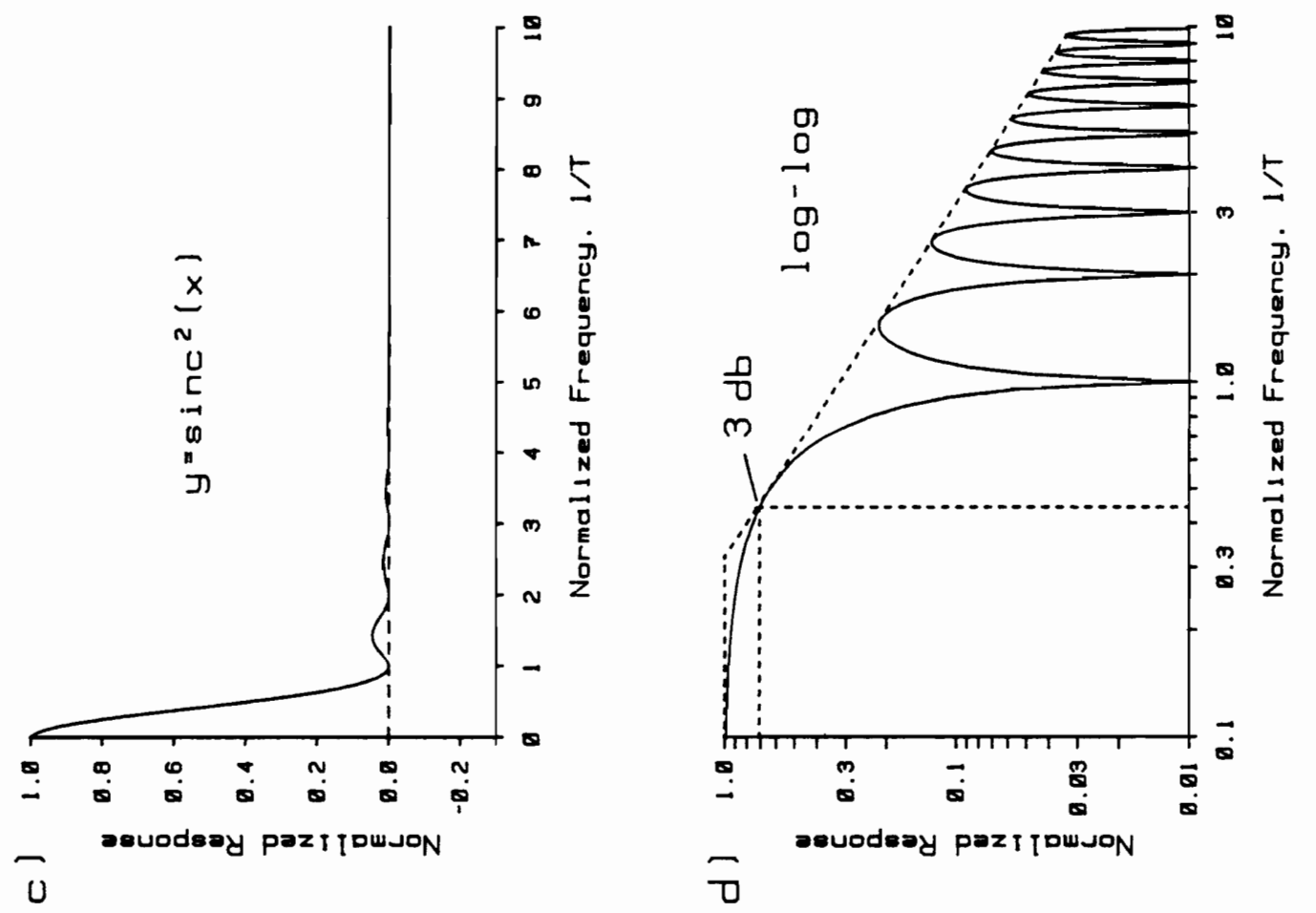

乎 岕

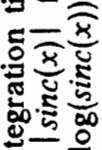
قैं

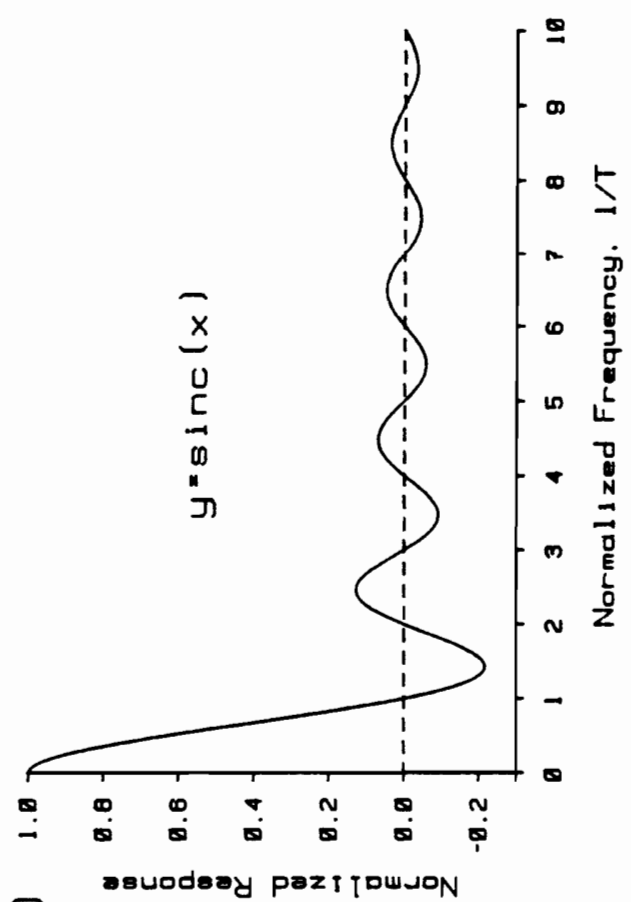

0 ซิ

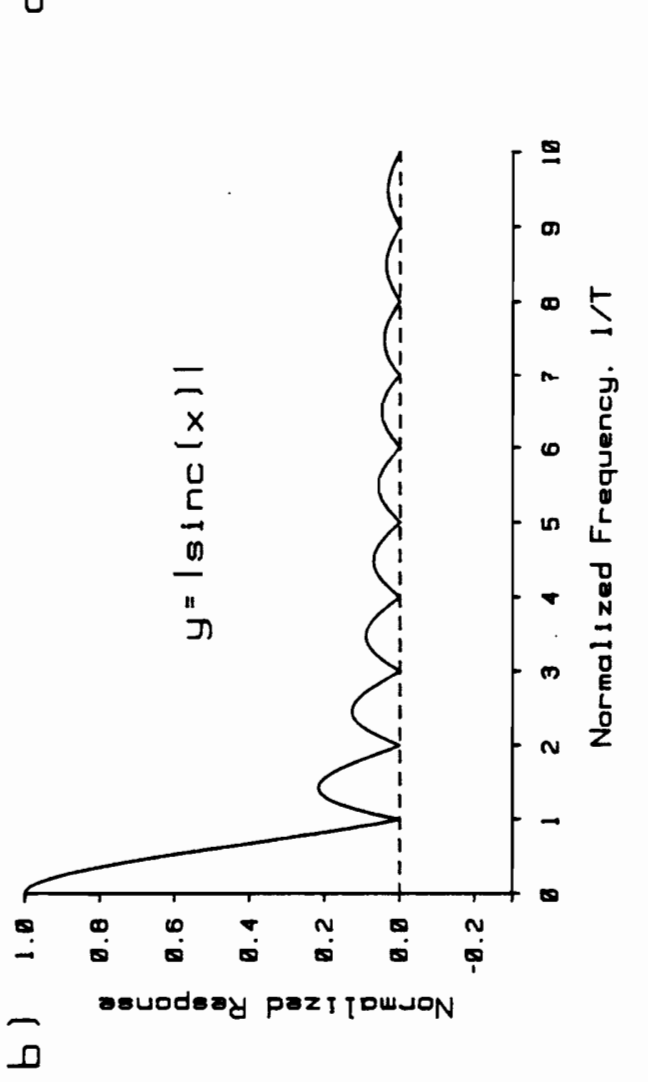

옹응 . Nis

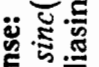
ธत क.

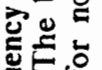
矛藏 ธํํำ 2. 등 잉 을 हू 온

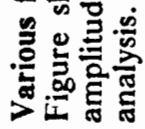
$\infty$ 壱 
$50 \%$ power attenuation and $70.7 \%$ amplitude attenuation point) is shown for reference. The worst-case rolloff is $-6 \mathrm{db} /$ octave or $-20 \mathrm{db} /$ decade, which is the attenuation rate of a first-order Butterworth filter. This worst-case performance is competent for a filter, and again, the PDA

performs much better in attenuating frequencies at or near $\bar{f}=n t_{i}$. The existence of this envelope, therefore, allowed the PDA to be used as its own anti-aliasing filter for coherent modulations as well.

\section{Frequency Response in MMS Design and Mirror/PDA Coordination}

Figure 69 shows the relative frequency ranges of the optical modulations $\left(\Delta \bar{f}_{x}\right)$, and the corresponding $\mathrm{AC}$ responses $\left(R_{\mathrm{x}}\right)$, for the MMS in the interferometric and the dispersive modes. In the design of the MMS, these frequency ranges were specified only after careful consideration of the effects on the detected signal.

\section{Interferometric Mode Response}

In the interferometric mode, the goal is to maintain the modulations produced by the interferometer, or maximize $R_{l}$. The optical range of the MMS is from 800 to $400 \mathrm{~nm}$. In the interferometric mode, this range of wavelengths produces the relative frequency range shown as $\Delta \bar{f}_{l}$ in Figure $69.800 \mathrm{~nm}$ corresponds to the low frequency, and $400 \mathrm{~nm}$ corresponds to the high frequency. The corresponding AC responses, indicted by f.R sub $<\mathrm{I}>$, range from 0.95 to 0.75 . This range of responses was obtained only after major modification of the MMS components. The modifications involved the following: 1) slowing the interferometer mirror down to lower the frequency of the light modulations, 2) speeding up the array readout to reduce $t_{i}$, and 3 ) electronically multiplying the $\mathrm{HeNe}$ laser reference signal by a factor of four to accurately coordinate the interferometer and the PDA scanning. 


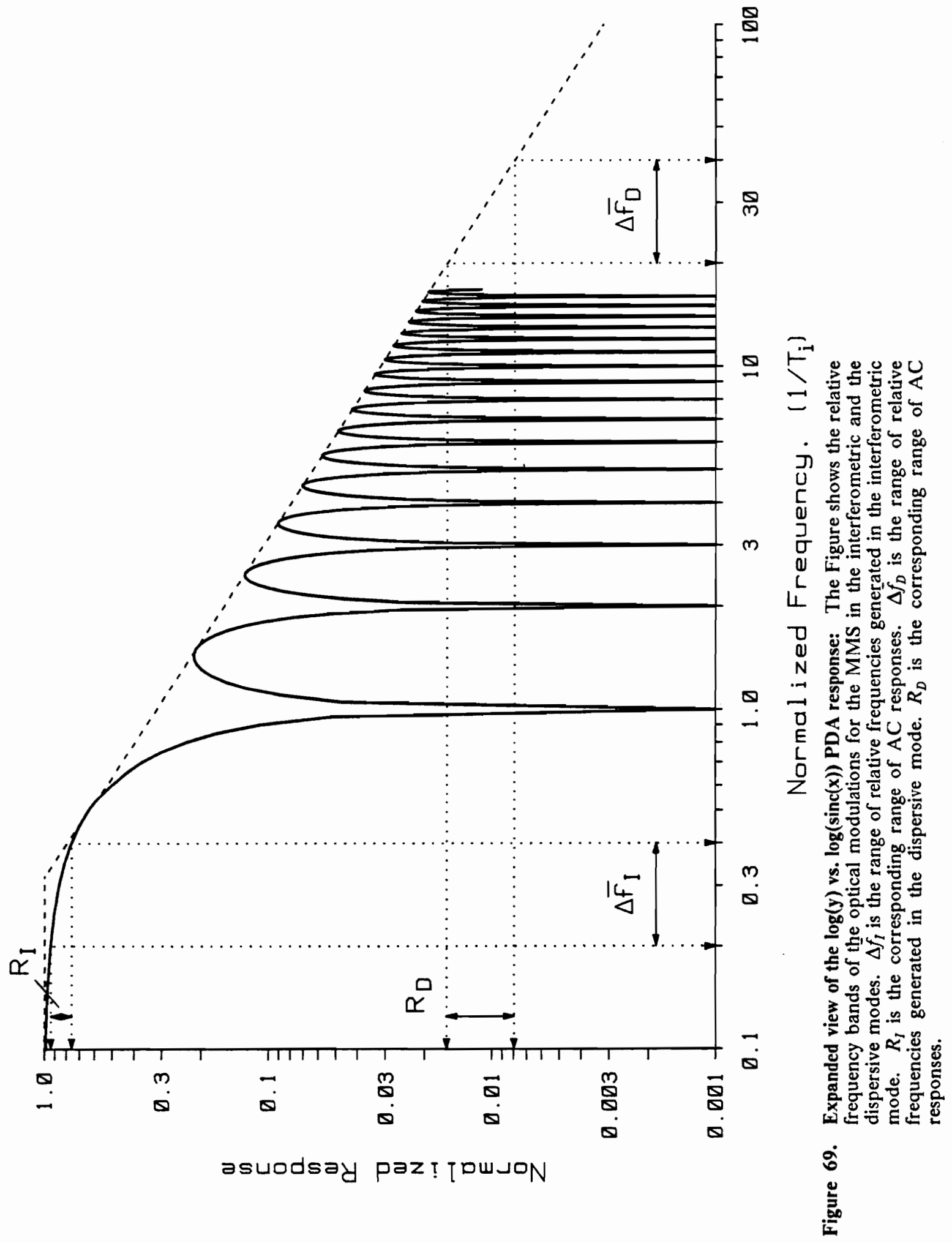


The need for these modifications can be shown by discussing the two earlier designs. Design I: In the original MMS design, one array scan was performed and one data point was taken for each HeNe fringe. This scheme made the range of $\Delta \bar{f}_{I}$ equal to 0.8 to 1.6 normalized frequency units. Referring to Figure 69, this range for $\Delta \bar{f}_{I}$ spans the first node of the sinc function, and leads to a serious attenuation of the $\mathrm{AC}$ response. Design II: The second design involved slowing the mirror down and speeding the array up, so that two array scans were performed and two data point were taken for each $\mathrm{HeNe}$ fringe. This scheme made $\Delta \bar{f}_{I}$ correspond to 0.4 to 0.8 normalized frequency units. Again referring to Figure 69 , this second range of $\Delta \bar{f}_{I}$ corresponds to normalized responses of approximately 0.75 to 0.25 . This response was judged to be inadequate.

The final design of the MMS resulted in an optical mirror velocity of $0.0506 \mathrm{~cm} / \mathrm{sec}$. This produces $\mathrm{HeNe}$ reference signal of $800 \mathrm{~Hz}$ and a multiplied reference signal of $3.2 \mathrm{kHz}$. This multiplied reference signal is used to initiate array scans, so $t_{i}$ in the interferometric mode is 3.125 msec. The $3.2 \mathrm{kHz}$ array scan rate, in turn, requires that the individual array pixels to be output at $2 \mathrm{MHz}$. Design of the analog electronics for this pixel rate was very difficult. Modifications to the MMS design, such as a longer array, a response in the UV, or shorter times for an interferometric data run, would push the pixel output rate even higher.

First Lobe of the Sinc Function: To further clarify the temporal response of the PDA and the MMS in the interferometric mode, a detailed plot of the first lobe of the $\operatorname{sinc}(f)$ function is shown in Figure 70. This Figure shows correspondence between the wavelengths and wavenumbers, the modulation frequencies produced by the interferometer, and the attenuation of the $\sin c(f)$. function. The MMS optical range and the AES wavelength range are specifically indicated. If the optical range of the MMS were extended into the UV, severe attenuation of these modulations would occur. Better interferometric response could be obtained by expand the $\sin c(f)$ envelope. This would require either speeding up the PDA readout rate, or by slowing the mirror down.

The portion of the $\mathrm{x}$-axis in Figure 70 from 0 to $1600 \mathrm{~Hz}$, and from 0 to $31,605 \mathrm{~cm}^{-1}$, corresponds directly to the unaliased interferometric spectra in Chapter 1. Also, this Figure is presented in the same format as the PDA wavenumber quantum efficiency curve, Figure 66. Total 

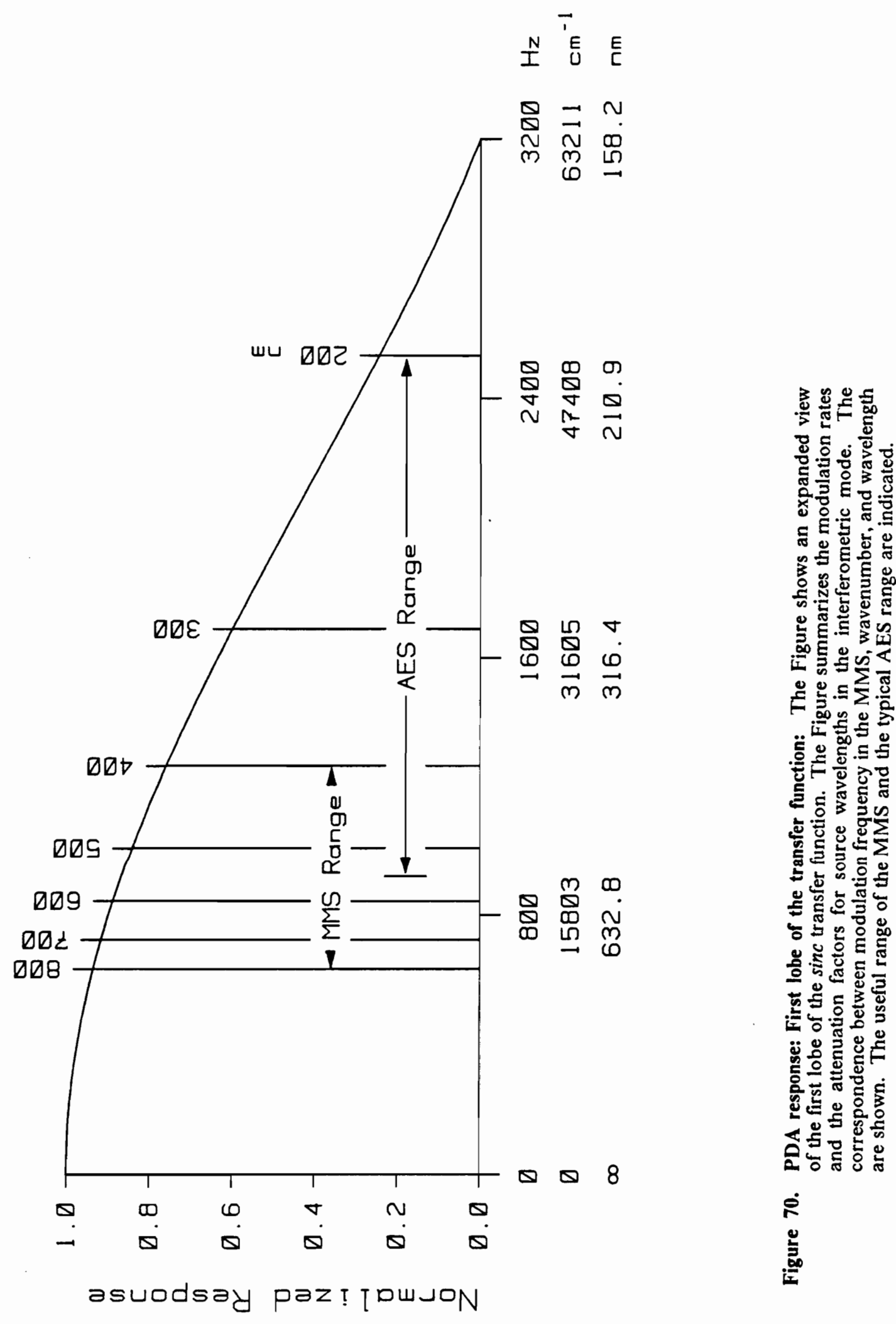
attenuation of the optical signal caused by the PDA in the interferometric mode is obtained from the multiplication of these two curves.

\section{Dispersive Mode Response}

In the dispersive mode, the goal is to suppress modulations caused by the interferometer, or minimize $R_{D}$. In an initial analysis of dispersive mode operation, the following conditions were found. The read rate of the individual pixels is limited to $15.6 \mathrm{kHz}$ by the computer. This in turn limits the array readout to $30 \mathrm{~Hz}$, making $t_{i}=33.3 \mathrm{msec}$. If the interferometer scans at the optical mirror velocity of $0.0506 \mathrm{~cm} / \mathrm{sec}, \Delta \bar{f}_{D}$ spans a range of 20 to 40 , and the worst-case values of $R_{D}$ range from 0.016 to 0.008 . $R_{D}$ could easily be reduced further by scanning the mirror faster. If a faster array readout were accomplished, the mirror would have to scan faster to maintain the low $R D$.

The final design of the MMS resulted in an optical mirror velocity of $0.0506 \mathrm{~cm} / \mathrm{sec}$. This produces $\mathrm{HeNe}$ reference signal of $800 \mathrm{~Hz}$ and a multiplied reference signal of $3.2 \mathrm{kHz}$. This multiplied reference signal is used to initiate array scans, so $t_{i}$ in the interferometric mode is 3.125 msec. The $3.2 \mathrm{kHz}$ array scan rate, in turn, requires that the individual array pixels to be output at $2 \mathrm{MHz}$. Design of the analog electronics for this pixel rate was very difficult. Modifications to the MMS design, such as a longer array, a response in the UV, or shorter times for an interferometric data run, would push the pixel output rate even higher. 


\section{Phase Errors in the MSS}

\section{Origins of Phase Errors}

By using the PDA as the detector in the interferometric mode, the MMS experiences two phase shifts not normally observed in an FT spectrometer. The first, discussed above, is the phase shift caused by an integrating detector. This phase shift is linear in frequency, and therefore wavenumber. For this shift, blue lines experience more delay and phase shift than red lines. The second phase shift is caused by the serial readout of the PDA. With the serial readout scheme, pixels at the beginning of the array are read almost immediately, whereas the pixels at the end are not available until just before the next START. Again, a delay in the time domain translates to a phase shift in the frequency domain. Because of the linear wavelength dispersion of the polychromator, distance across the array corresponds to wavelength. Consequently, this second phase shift is linear in wavelength.

\section{Partial Compensation of Phase Errors}

In the MMS, the two phase errors were made to partially compensate for one another. The PDA could have been mounted in the focal plane of the polychromator to yield a blue-to-red readout order, or a red-to-blue readout order. By choosing the blue-to-red readout order, the red lines were made to experience a greater readout-time delay, and thus the phase shift is greater for red lines than for blue For the integration-time phase shift, the shift is greater for blue lines than for red. The combination of the two phase shifts in this manner produced the most constant phase shift across the spectrum. 


\section{Noise and Errors within the PDA}

The noise and errors of the PDA are discussed for two reasons. First, the MMS was detector noise limited. Second, the discussion helps point avenues of future improvements of the MMS.

There are three sources of error within solid state detectors: readout noise, dark current shot noise, and fixed pattern signal. ${ }^{19,17,120}$ Fixed pattern signal is a nonrandom pixel- to-pixel variation in signal, so it is not considered a noise; see below. Dark current noise and readout noise are random. The total noise in the PDA is therefore given by

$$
n_{T}=\left(n_{r}^{2}+n_{d}^{2}\right)^{1 / 2}
$$

where $n_{T}$ is the total noise, $n_{r}$ is the readout noise, and $n_{d}$ is the dark current noise. Each of these noises is given in electrons.

Two of the errors, readout noise and fixed pattern signal, are attributable to the relatively large video line capacitance and clock line capacitance found in a PDA; see Table 26. For PDAs, readout noise is generally the limiting noise.

\section{Readout Noise and Video Line Capacitance}

Readout noise or kTC noise is due to the uncertainty in resetting a capacitance to a fixed voltage. In the PDA, the capacitance is formed by the pixel itself and the video lines. The readout noise is given by

$$
n_{r}=\left(1 / q_{e}\right)\left(k T C_{T}\right)^{1 / 2}
$$

and the total capacitance is given by

$$
C_{T}=C_{d}+C_{V}
$$


where $n_{r}$ is the readout noise in electrons, $q_{e}$ is the charge on the electron, $k$ is Boltzmann's constant, $T$ is the absolute temperature, $C_{T}$ is the total capacitance, $C_{d}$ is the individual diode capacitance, and $C_{V}$ is the video line capacitance.

The majority of the readout noise in a PDA is attributable to the relatively large video line capacitance, $C_{V}$. See Figure 64 and Table 26. The capacitance results from the combination of the long video line and the connected FET regions versus the chip substrate. Off-chip circuitry also adds to this capacitance, due to the capacitance of external amplifiers leads. The on-chip video line capacitance is typically $12 \mathrm{pf}$ per video line. Readout noise is particularly troublesome because it is not very amenable to cooling. Cooling the array from +25 to $-40^{\circ} \mathrm{C}$ reduces readout noise only by $15 \%$.

CIDs and particularly CCDs have much lower video line capacitances, and are thus much less susceptible to readout noise. CCDs, for example, move the accumulated photocharge across the chip in an analog form from the pixel to an on-chip amplifier. The CCDs, therefore eliminate the video line and off-chip capacitances.

Readout noise of a PDA versus a CCD can best be appreciated by calculating the number of detected photons required for photon noise to equal readout noise. In PDAs, the readout noise is $1,200 \mathrm{e}^{-}$in a good system. Therefore the number of detected photons required in this case is $(1,200)^{2}$, or about $1,500,000$ photons. In CCDs by contrast, the readout noise is as low as $4 \mathrm{e}^{-}$per element. The number of detected photons required in this case is $4^{2}$, or about 16 photons. CCDs are obviously much better for low-light, low-noise detection systems.

\section{Dark Current and Dark Current Noise}

Dark current is the thermally generated leakage current at the diodes. It has three effects: it introduces a dark current background, it introduces dark current shot noise, and it limits integration time. The dark current in the RL-0512S is a strong function of temperature, being 
halved for every $7^{\circ} \mathrm{C}$ drop in temperature. It can therefore be reduced significantly by moderate cooling. The dark current as a function of temperature is found empirically.

Because the dark current obeys Poisson statistics, the dark current noise is proportional to the square root of the dark current. The noise is given by 19

$$
n_{d}=\left(\frac{i_{d} t_{i}}{q_{e}}\right)^{1 / 2}
$$

where $n_{d}$ is the dark current shot noise in electrons, $i_{d}$ is the average dark current, $t_{i}$ is the integration time, and $q_{c}$ is the charge on the electron.

\section{Fixed Pattern Signal}

Fixed pattern signal is a regular pixel-to-pixel variation in the dark signal baseline, and is repeatable from scan to scan. One cause is coupling of clock transitions into the video lines. A second cause is the integrated dark current. In the $\mathrm{S}$ series arrays, fixed pattern signal is reduced by a differential readout scheme. Pairs of active and dummy diodes are accessed simultaneously; see Figure 64. Variations along the array should be seen by both diodes. The variations can then be removed by a differential amplifier. 


\section{Bibliography}

1. P. Hobbs, D. E. M. Spillane, R. D. Snook, and A. P. Thorne, J. Anal. At. Spectrom. 3, 543-546 (1988).

2. G. Horlick and E. G. Codding, Anal. Chem. 45, 1490-1493 (1973).

3. M. B. Denton, H. A. Lewis, and G. R. Sims, in Multichannel Image Detectors, Y. Talmi, Ed. (American Chemical Society, Washington, D.C., 1983), Vol. 2, pp. 133-154.

4. G. R. Sims and M. B. Denton, in Multichannel Image Detectors, Y. Talmi, Ed. (American Chemical Society, Washington, D.C., 1983), Vol. 2, pp. 117-132.

5. G. Horlick, Appl. Spectrosc. 30, 113-123 (1976).

6. S. Lepla and G. Horlick, Appl. Spectrosc. 43, 1187-1195 (1989).

7. Y. Talmi and R. W. Simpson, Appl. Opt. 19, 1401-1414 (1980).

8. F. Grabau and Y. Talmi, in Multichannel Image Detectors, Y. Talmi, Ed. (American Chemical Society, Washington, D.C., 1983), Vol. 2, pp. 75-116.

9. R. B. Bilhorn, J. V. Sweedler, P. M. Epperson, and M. B. Denton, Appl. Spectrosc. 41, 1114-1125 (1987).

10. R. B. Bilhorn, P. M. Epperson, J. V. Sweedler, and M. B. Denton, Appl. Spectrosc. 41, 1125-1136 (1987).

11. G. M. Levy, A. Quaglia, R. E. Lazure, and S. W. McGeorge, Spectrochim. Acta 42B, 341-351 (1987).

12. R. B. Bilhorn and M. B. Denton, Appl. Spectrosc. 49, 1-11 (1989).

13. Z. Walker and M. W. Blades, Spectrochim. Acta 41B, 761-775 (1986). 
14. M. C. Edelson, "High resolution plasma spectrometry," in Inductively Coupled Plasma in Analytical Atomic Spectrometry, A. Montaser and D. W. Golightly, Eds. (VCH Publishers, New York, 1987) Chap. 7, p. 242.

15. P. W. J. M. Boumans and J. J. A. M. Vrakking, Spectrochim. Acta 39B, 1239- 1260 (1984).

16. A. P. Thorne, J. Anal. At. Spectrom. 2, 227-232 (1987).

17. Reticon Corp., Image Sensing Products: 1986 Catalog (EG\&G Reticon, Sunnyvale CA, 1986) p.(1-33).

18. R. K. Winge, V. A. Fassel, and M. C. Edelson, Spectrochim. Acta 43B, 85-91 (1988).

19. R. W. Simpson, Rev. Sci. Instrum. 50, 730-732 (1979).

20. R. K. Winge, V. A. Fassel, and D. E. Eckels, Appl. Spectrosc. 40, 461-464 (1986).

21. G. Horlick and W. K. Yuen, Anal. Chem. 47, 775A-780A (1975).

22. G. Horlick, R. H. Hall, and W. K. Yuen, "Atomic Emission Spectrochemical Measurements with a Fourier Transform Spectrometer" in Fourier Transform Infrared Spectroscopy, J. R. Ferraro, L. J. Basile, and A. Mantz, Eds. (Academic Press, New York, 1982) Vol. 3, pp. 37-81.

23. R. C. L. Ng and G. Horlick, Appl. Spectrosc. 39, 834-840 (1985).

24. R. C. L. Ng and G. Horlick, Appl. Spectrosc. 39, $841-847$ (1985).

25. E. A. Stubley and G. Horlick, Appl. Spectrosc. 39, 800-804 (1985).

26. E. A. Stubley and G. Horlick, Appl. Spectrosc. 39, 805-810 (1985).

27. E. A. Stubley and G. Horlick, Appl. Spectrosc. 39, $811-817$ (1985).

28. S. Marra and G. Horlick, Appl. Spectrosc. 40, 804-813 (1986).

29. L. M. Faires, B. A. Palmer, R. Engleman, Jr., and T. M. Niemczyk, Spectrochim. Acta 39B, 819-828 (1984).

30. L. M. Faires, B. A. Palmer, and J. W. Brault, Spectrochim. Acta 40B, 135- 143 (1985).

31. L. M. Faires, B. A. Palmer, R. Engleman, Jr., and T. M. Niemczyk, Spectrochim. Acta 40B, 545-551 (1985).

32. L. M. Faires, Spectrochim. Acta 40B, 1473-1483 (1985).

33. L. M. Faires, Anal. Chem. 58, 1023A-1034A (1986).

34. L. M. Faires, J. Anal. At. Spectrom. 2, 585-590 (1987).

35. A. P. Thome, Anal. Proc. 22, 63-65 (1985).

36. A. P. Thorne, C. J. Harris, I. Wynne-Jones, R. C. M. Learner, and G. Cox, J. Phys. E: Sci. Instrum. 20, 54-60 (1987). 
37. D. E. M. Spillane, R. D. Snook, A. P. Thorne, and J. E. G. Wheaton, J. Anal. At. Spectrom. 2, 591-594 (1987).

38. J. W. Brault, J. Opt. Soc. Am. 66, 1081 (1976).

39. J. W. Brault, "High Resolution Fourier Transform Spectroscopy" in High Resolution in Astronomy, Proceedings of the 15th Advanced Course of the Swiss Society of Astronomy and Astrophysics, A. Benz, M. Huber, M. Mayor, Eds. (Geneva Observatory, Sauverny, Switzerland, 1985), Vol. 15, pp. 1-70.

40. B. A. Palmer, R. Engleman, Jr., L. Testerman, and J. Brault, Proc. SPIE 380, 68-69 (1983).

41. M. L. Parsons and B. A. Palmer, Spectrochim. Acta 43B, 75-78 (1988).

42. F. D. Kahn, Astrophys. J. 129, 518-521 (1959).

43. F. W. Plankey, T. H. Glenn, L. P. Hart, and J. D. Winefordner, Anal. Chem. 46, 1000-1005 (1974).

44. T. L. Chester, J. J. Fitzgerald, and J. D. Winefordner, Anal. Chem. 48, 779-783 (1976).

45. T. Hirschfeld, Appl. Spectrosc. 30, 68-69 (1976).

46. T. Hirschfeld, Appl. Spectrosc. 30, 234-236 (1976).

47. L. Mertz, Transformations in Optics (John Wiley and Sons, New York, 1965), p. 10.

48. E. Voigtman and J. D. Winefordner, Appl. Spectrosc. 41, 1182-1184 (1987).

49. R. M. Belchamber, G. Horlick, Spectrochim. Acta 37B, 17-27 (1982).

50. J. M. Brault, 1988, private communication.

51. R. M. Belchamber and G. Horlick, Spectrochim. Acta 37B, 71-74 (1982).

52. G. M. Hieftje, 1988, private communication.

53. R. M. Belchamber and G. Horlick, Spectrochim. Acta 37B, 1037-1046 (1982).

54. S. A. Myers and D. H. Tracy, Spectrochim. Acta 38B, 1227-1253 (1983).

55. M. A. Marks and G. M. Hieftje, Appl. Spectrosc. 42, 277-280 (1988).

56. E. D. Salin and G. Horlick, Anal. Chem. 52, 1578-1582 (1980).

57. G. L. Walden, J. N. Bower, S. Nikdel, D. L. Bolton, and J. D. Winefordner, Spectrochim. Acta 35B, 535-546 (1980).

58. N. Furuta, C. A. Monnig, P. Yang, and G. M. Hieftje, Spectrochim. Acta 44B, 649-655 (1989).

59. P. R. Griffiths and J. A. de Haseth, Fourier Transform Infrared Spectrometry (John Wiley and Sons, New York, 1986).

60. L. Mertz, Transformations in Optics (John Wiley and Sons, New York, 1965), p. 36-37. 
61. E. O. Brigham, The Fast Fourier Transform and Its Applications (Prentice Hall, Englewood Cliffs, N. J., 1988).

62. G. L. Long and L. D. Perkins, Appl. Spectrosc. 41, 980-985 (1987).

63. L. Matus, C. Boss, and A. N. Riddle, Rev. Sci. Instr. 54, 1667-1673 (1983).

64. C. I. M. Beenakker, Spectrochim. Acta 31B, 483-486 (1976).

65. R. Lysakowski, Development of a Low-Power Microwave Plasma and Its Application as an Atom Source for Atomic Spectroscopy, Ph.D. dissertation, VPI\&SU, 1987.

66. L. R. Layman and F. E. Lichte, Anal. Chem. 54, 638-642 (1982).

67. G. R. Harrison, MIT Wavelength Tables, (The MIT Press, Cambridge, Massachusetts, 1969), Vol. 1.

68. F. M. Phelps III, MIT Wavelength Tables, (The MIT Press, Cambridge, Massachusetts, 1982), Vol. 2.

69. S. W. McGeorge, 1988, private communication.

70. P. Luc and S. Gerstenkom, Appl. Opt. 17, 1327-1331 (1978).

71. P. W. J. M. Boumans, "Basic Concepts and Characteristics of ICP-AES," in Inductively Coupled Plasma in Analytical Atomic Spectrometry. Part 1: Methodology,

Instrumentation, and Performance, P. W. J. M. Boumans, Ed. (John Wiley and Sons, New York, 1987) Chap. 4, pp 100- 257.

72. E. V. Loewenstein, Aspen International Conference on Fourier Spectroscopy, 1970, G. A. Vanasse, A. T. Stair, Jr., and D. J. Baker, Eds. (Air Force Cambridge Research Laboratories, AFCRL-71-0019, Special Report 114, 1971) pp. 3-17.

73. E. L. Dereniak and D. G. Crowe, Optical Radiation Detectors (John Wiley and Sons, New York, 1984), p 7.

74. J. W. Olesik, "Spectrometers," in Inductively Coupled Plasma in Analytical Atomic Spectrometry. Part I: Methodology, Instrumentation, and Performance, P. W. J. M. Boumans, Ed. (John Wiley and Sons, New York, 1987) Chap. 8, pp 466-535.

75. D. E. Smith, D. C. Liang, D. Steel, and M. W. Blades, "Analytical Characteristics of Furnace Atomization Plasma Emission Spectrometry (FAPES)," Spectrochim. Acta 45B, (1990) in press.

76. D. C. Liang and M. W. Blades, "An atmospheric pressure capacitively coupled plasma formed inside a graphite furnace as a source for atomic emission spectroscopy," Spectrochim. Acta 45B, (1990) in press.

77. R. E. Sturgeon, S. N. Willie, V. Luong, S. S. Berman, and J. G. Dunn, "Furnace Atomization Plasma Emission Spectrometry - FAPES," J. Anal. At. Spectrom. 5, (1990) in press.

78. J. M. Harnly, D. L. Styris, and N. E. Ballou, "Furnace Atomic Non-thermal Excitation Spectrometry with the Furnace as a Hollow Anode," Anal. At. Spectrom. 5, (1990) in press. 
79. H. Falk, E. Hoffmann, and Ch. Ludke, Spectrochim. Acta 36B 767-771 (1981).

80. H. Falk, E. Hoffmann, and Ch. Ludke, Prog. Analyt. Spectrosc. 11, 417-480 (1988).

81. R. D. Snook and C. M. Du, J. Anal. At. Spectrom. 3, 433-440 (1988).

82. J. Davies and R. D. Snook, J. Anal. At. Spectrom. 2, 27 (1987).

83. J. Davies and R. D.Snook, J. Anal. At. Spectrom. 1, 195 (1986).

84. H. Tan, S. Chan, and A. Montaser, Anal. Chem. 60, 2542-2544 (1988).

85. P. R. Fielden, M. Jiang, and R. D. Snook, Appl. Spectrosc. 43, 1444-1449 (1987).

86. M. L. Bruce, J. M. Workman, J. A. Caruso, and D. J. Lahti, Appl. Spectrosc. 39, 935-942 (1985).

87. L. D. Perkins and G. L. Long, Appl. Spectrosc. 43, 499-504 (1989).

88. Q. Jin, C. Zhu, K. Brushwyler, and G. M. Hieftje, Appl. Spectrosc. 44, 183-186 (1985).

89. T. Cecconie, S. Muralidharan, and H. Freiser, Appl. Spectrosc. 42, 177- 179 (1988).

90. J. A. Koropchak and D. H. Winn, Appl. Spectrosc. 41, 1311-1318 (1987).

91. S. R. Goode and K. W. Baughman Spectrochim. Acta 38B, 75 (1983).

92. J. V. Sweedler, R. D. Jalkian, G. R. Sims, and M. B. Denton, Appl. Spectrosc. 44, 14-20 (1990).

93. A. F. Parisi, G. D. Rayson, G. M. Hieftje, and J. W. Olesik, Spectrochim. Acta 42B, 361-376 (1987).

94. L. M. Faires, T. M. Bieniewski, C. T. Apel, and T. M. Niemczyk, Appl. Spectrosc. 39, 5-9 (1985).

95. D. R. Demers, Appl. Spectrosc. 33, 584 (1979).

96. Y. Talmi, T. Crosmun, N. M. Larson, Anal. Chem. 48, 32 (1976).

97. R. D. Deutsch and G. M. Hieftje, Appl. Spectrosc. 39, 19-24 (1985).

98. R. K. Winge, D. E. Eckels, E. L. DeKalb, and V. A. Fassel, J. Anal. At. Spectrom. 3, 849-855 (1988).

99. G. A. Vanasse, A. T. Stair, Jr., and D. J. Baker, Eds. The Aspen International Conference on Fourier Spectroscopy, 1970, (Air Force Cambridge Research Laboratories, AFCRL-71-0019, Special Report 114, 1971).

100. "Méthodes Nouvelles de Spectroscopie Instrumentale", Colloques Internationaux du Centre National de lal Recherche Scientifique, No. 161 Orsay, 1966 (CNRS, Paris, 1967). Papers in French and English.

101. New Methods of Instrumental Spectroscopy: Proceedings of CNRS International Symposium No. 161, (Gordon and Breach, New York, 1971). Translations of the French papers from "Méthodes Nouvelles de Spectroscopie Instrumentale", Colloques 
Internationaux du Centre National de lal Recherche Scientifique, No. 161 Orsay, 1966 (CNRS, Paris, 1967).

102. Selected original papers from "Méthodes Nouvelles de Spectroscopie Instrumentale", Colloques Internationaux du Centre National de lal Recherche Scientifique, No. 161 Orsay, 1966 (CNRS, Paris, 1967). published in J. Phys. (Supplement), 28,C2 (1967)

103. E. O. Brigham, The Fast Fourier Transform (Prentice Hall, Englewood Cliffs, N. J., 1974).

104. R. N. Bracewell, The Fourier Transform and Its Applications (McGraw-Hill, New York, 1965).

105. R. N. Bracewell, The Fourier Transform and Its Applications (McGraw-Hill, New York, 1978), 2nd ed.

106. J. Chamberlain, The Principles of Interferometric Spectroscopy (John Wiley and Sons, New York, 1979).

107. J. W. Cooley and J. W. Tukey, Math. Comput. 19, 296 (1965).

108. R. J. Nordstrom, "Aspects of Fourier Transform Visible/UV Spectroscopy," in Fourier, Hadamard, and Hilbert Transforms in Chemistry, A. G. Marshall, Ed. (Plenum Press, New York, 1982) pp 421-452.

109. R. H. Norton and R. Beer, J. Opt. Soc. Am. 66, 259-264 (1976).

110. J. K. Kauppinen, D. J. Moffat, D. G. Cameron, and H. H. Mantsch, Appl. Opt. 20, 1866-1879 (1981).

111. I. Wynne-Jones, Infrared Phys. 24, 273-275 (1984).

112. J. W. Brault, Mikrochim. Acta 1987, 215-227 (1987).

113. H. Sakai and G. A. Vanasse, J. Opt. Soc. Am. 56, 131-132 (1966).

114. H. Sakai, "High Resolving Power Fourier Spectroscopy," in Spectrometric Techniques, vol l., G. A. Vanasse, Ed. (Academic Press, New York, 1977).

115. S. W. McGeorge, Spectroscopy, 2, 26-32 (1987).

116. H. Tseng, J. R. Ambrose, and M. Fattahi, Journ. Imag. Sci., 29, 1-7 (1985).

117. Reticon Corp. Application Note no. 121: "Spectral Response of Reticon Linear Photodiode Arrays" pp 9-12 (1980).

118. W. McGeorge and E. D. Salin, Spectrochim. Acta 40B, 435-445 (1985).

119. T. Okamato, S. Kawata, and S. Minami, Appl. Opt. 23, 267 (1984).

120. S. S. Vogt, R. G. Tull, and P. Kelton, Appl. Opt., 17, 574-592 (1978). 


\section{Vita}

Mark Andrew Wingerd was born in Pittsburgh, Pennsylvania, on March 6, 1953, to John and Vera Wingerd. Mark attended Slippery Rock State College, and was awarded a B.S. in Chemistry in 1978. On September 2, 1978, Mark married Diane Horm-Wingerd in Beaver Falls, Pennsylvania. Mark and Diane entered graduate school at VPI\&SU on September 25, 1978. In March, 1990, Mark accepted a position as Principal Scientist with Thermo Jarrel Ash in Franklin, Massachusetts. He will be joining Diane, who is an Assistant Professor in Child Development at The University of Rhode Island. 